\title{
THREE ESSAYS ON THE ADVERSE EFFECTS OF WEATHER EXTREMES ON LOCAL ECONOMIC AND HEALTH OUTCOMES IN INDONESIA
}

BY

ADITYA KUSUMA

\author{
A thesis \\ submitted to the Victoria University of Wellington \\ in fulfillment of the requirements for the degree of \\ Doctor of Philosophy in Economics
}

Victoria University of Wellington

(2019) 


\section{Table of Contents}

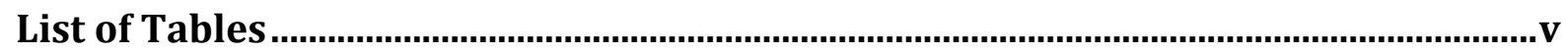

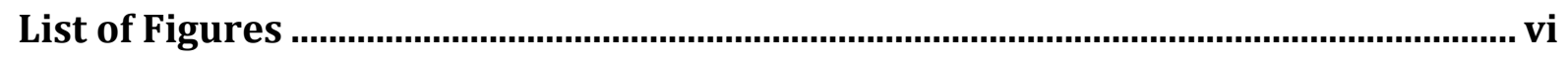

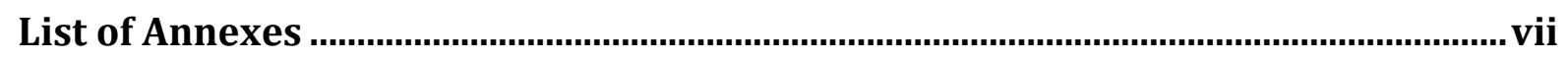

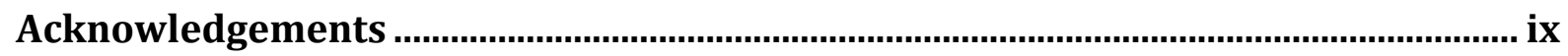

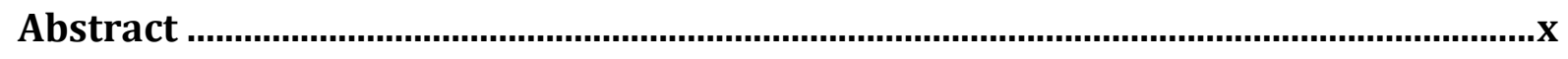

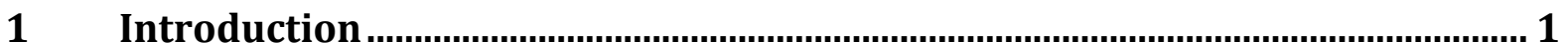

2 A Viable and Cost-Effective Weather Index Insurance for Rice in Indonesia. 4

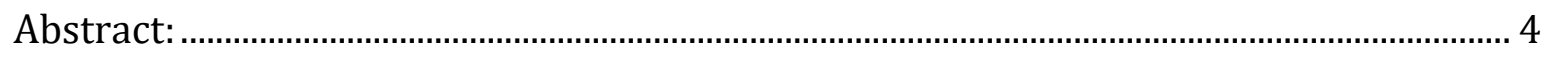

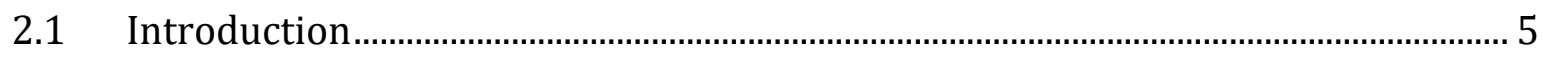

$2.2 \quad$ Background and motivation ........................................................................................... 7

2.2.1 Weather Index Insurance: Empirical Research ......................................................... 8

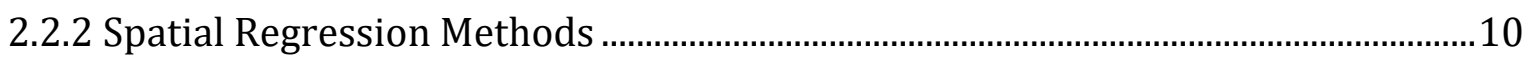

2.2.3 The Study Area: Indonesian Districts .........................................................................11

2.2.4 About WII in Indonesia ...........................................................................................

2.3 Research questions, data, and methods...................................................................

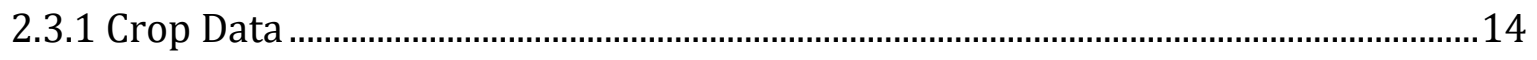

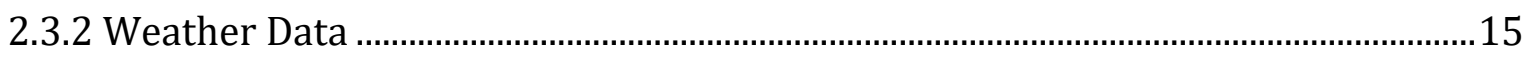

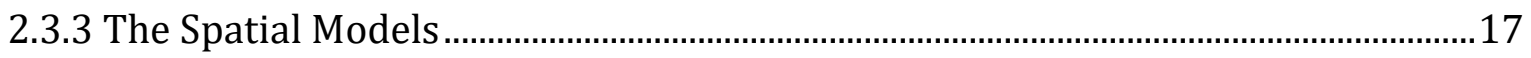

2.3.4.1 Fixed Effects, Spatial Auto-Regressive, and Spatial Error Models ................17

2.3.4.2 Geographically Weighted Regression (GWR).................................................18

2.3.4 Actuarial Analysis: Design and Valuation of index insurance..................................21

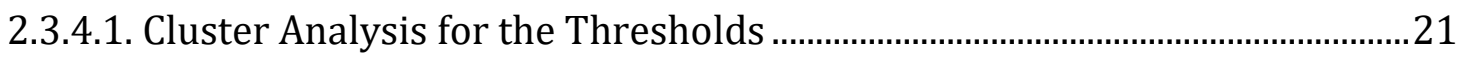

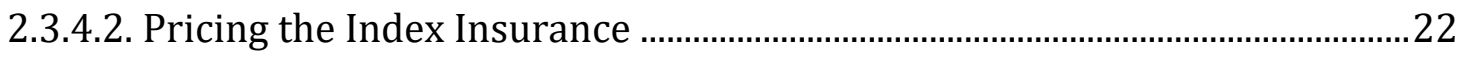

2.3.5 Efficiency Test to Measure WII's Risk Reduction Capability …….............................25

2.4 Result: spatially varying relationship of weather and paddy yield ......................26

2.5 Designing a weather index insurance product ….................................................... 31 
2.6 The efficiency of weather index insurance using the PDSI .33

2.7 Conclusion, caveats, and recommendations .37

Annexes Chapter TWO 40

3 Drought Consequences on Households: Exploring Evidence from

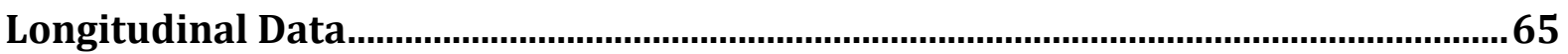

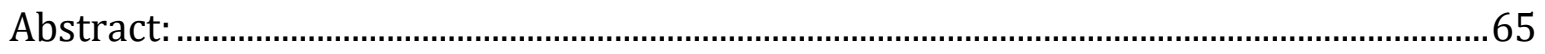

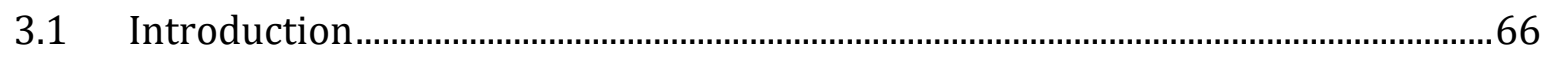

3.2 Macroeconomic and weather conditions in Indonesia.............................................68

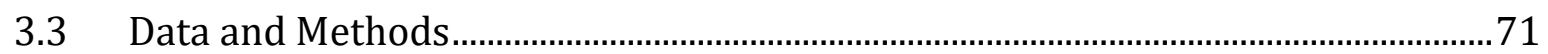

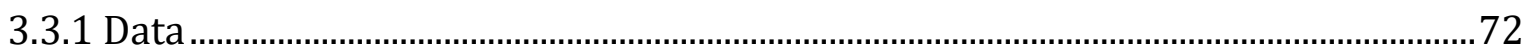

3.3.1.1 Weather data and constructions of drought events ........................................72

3.3.1.2 Household longitudinal data (Indonesian Family Life Surveys/IFLS) ........74

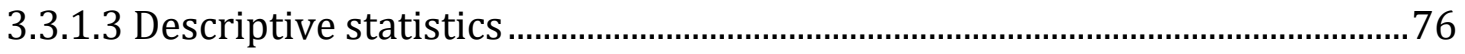

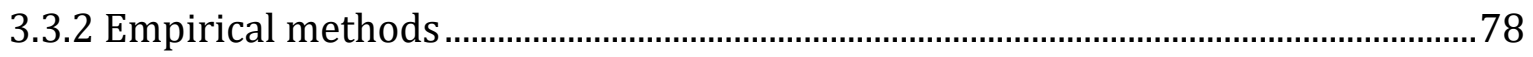

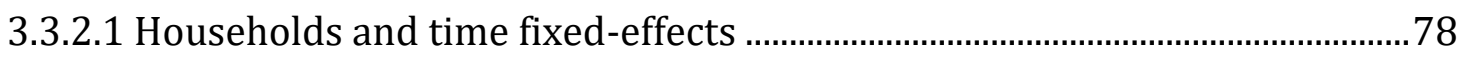

3.3.2.2 Robust standard errors for cross-sectional dependence and

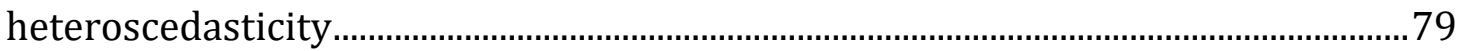

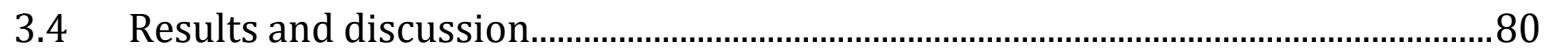

3.4.1 The Effect of weather shocks on the household economy ..........................................81

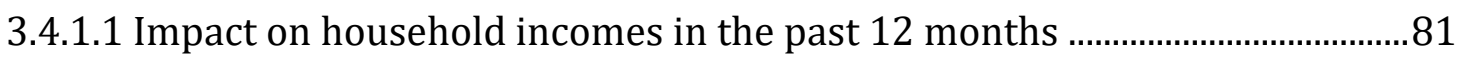

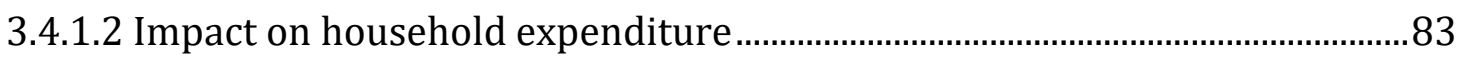

3.4.2 The effect of droughts on the outcomes of different types of households ............85

3.4.2.1 Drought impacts on the income of the poor, average and rich households

3.4.2.2 Drought impact on the monthly expenditure of the poor, average and rich households 89

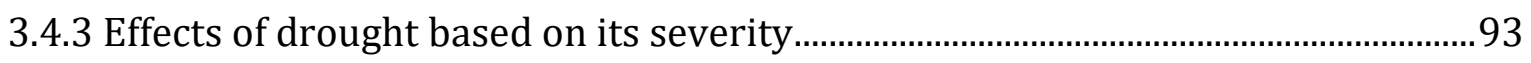

3.4.3.1 Consequences of moderate, severe and extreme droughts on income .......93

3.4.3.2 Impacts on household expenditures based on drought severity...................95 


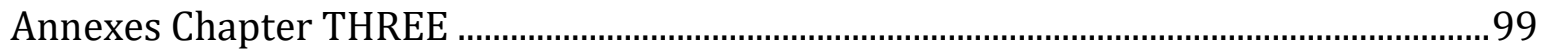

4 The Effects of Extreme Weather on Adults' Health and Well-being ............. 104

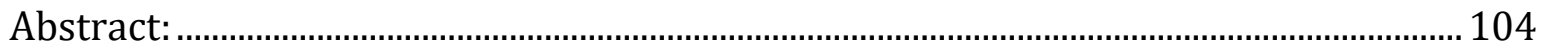

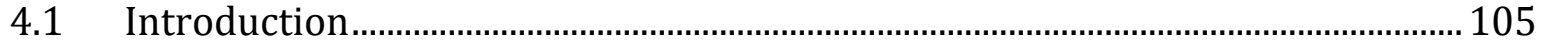

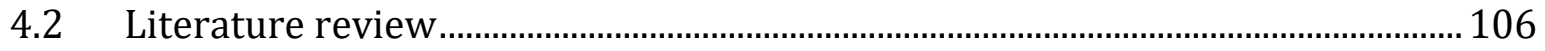

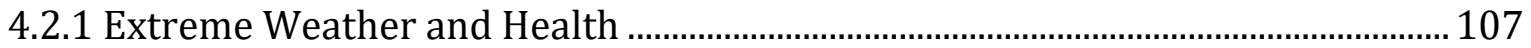

4.2.2 The research design in the weather-health literature .............................................. 108

4.2.3 Literature progress and gaps in low and low to middle-income countries....... 110

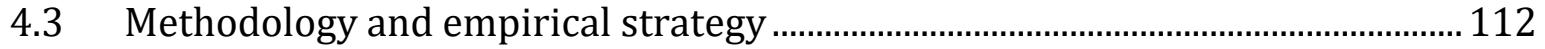

4.4 Weather Data and Socioeconomic statistics ......................................................... 115

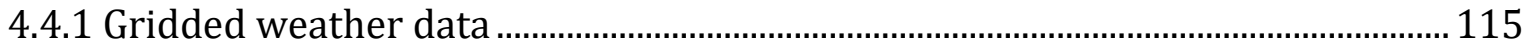

4.4.2 Socioeconomic indicators of an individual in the Indonesian Family Life Survey

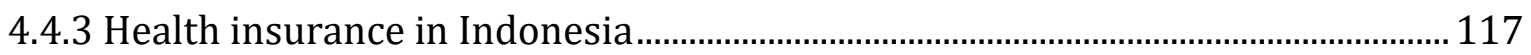

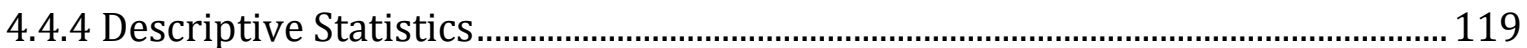

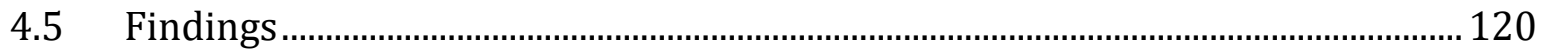

4.5.1 Marginal effects of extreme weather and symptoms of illness ............................. 120

4.5.2 Extreme rainfall effects on other health-related outcomes .................................... 122

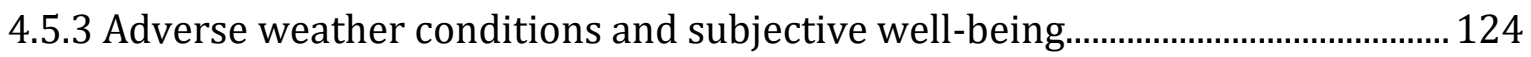

4.5.4 Individual and household features as coping strategies to reduce health and

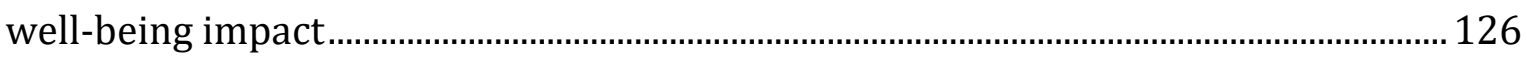

4.5.4.1 Reviews on all coping strategies ……............................................................ 126

4.5.4.2 Reviews on each coping strategies ................................................................ 129

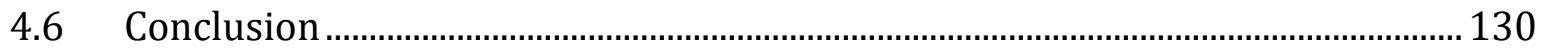

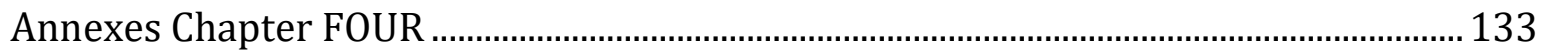

$5 \quad$ CONCLUSION

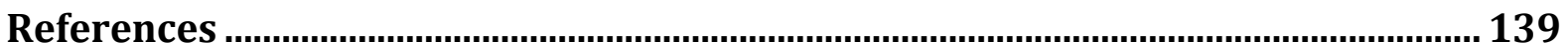




\section{LIST OF TABLES}

Table 1 Summary Statistics Data (428 districts in 24 years) 16

Table 2 Estimation Results Fixed Effect, Spatial Regression Model and GWR Panel of 428 Districts in Indonesia during 1990 - 2013

Table 3 Parameters of Weather Index Insurance Using Drought Indices PDSI for Rice Production in Major Islands in Indonesia .33

Table 4 Comparison revenue with and without insurance in selected districts .35

Table 5 Efficiency test using mean-semivariance model .36

Table 6 Descriptive statistics main variables of all sample IFLS households .77

Table 7 Summary of statistical checks .80

Table 8 Effects of drought shocks on household incomes .82

Table 9 Effects of drought shocks on household expenditures. .83

Table 10a Impact of droughts on the incomes of poor households .86

Table 10b Impact of droughts on the incomes of average households .87

Table 10c Impact of droughts on the incomes of rich households .88

Table 11a Impact of droughts on the monthly expenditure of poor households .90

Table 11b Impact of droughts on the monthly expenditure of average households...91

Table 11c Impact of droughts on the monthly expenditure of rich households .92

Table 12 Effects of moderate, severe and extreme drought on annual income. .94

Table 13 Effects of drought on household expenditures relative to its severity. .96

Table 14 Summary statistics weather events and individual characteristics 119

Table 15 Average marginal effects on symptoms of illness 121

Table 16 Health-related outcome impacts due to extreme weather. 123

Table 17 Life satisfaction impacts due to extreme weather 125

Table 18 The vulnerability of weather effects on health-related outcomes 126

Table 19 Marginal effect of the economic condition against dry month episodes .... 128 


\section{LIST OF FIGURES}

Figure 1 Spatial distribution of GWR panel coeff. estimate PDSI planting season........28

Figure 2 Spatial distribution of local coeff. estimate PDSI planting season of districts that are pseud-significant at $5 \%$ significance level

Figure 3 Spatial distribution of GWR panel coefficient estimate PDSI planting season of districts that are pseudo-significant at $5 \%$ significance level.

Figure 4 Spatial distribution of GWR panel coefficients PDSI in planting season of districts in major islands that are pseudo-significant at $5 \%$ significance level 30

Figure 5 Trigger of underlying index PDSI for districts in the island of Sulawesi, Sumatra, Kalimantan and Papua using model-based cluster. 32

Figure 6 Paddy revenues without and with index insurance in selected districts in Sulawesi, Kalimantan, and Sumatera 34

Figure 7 Macroeconomic Statistics Indonesia 1970 - 2016 .69

Figure 9 Study area and gridded weather data 116

Figure 10 Effect of economic condition to reduce dry month episodes 129 


\section{LIST OF ANNEXES \\ Annexes Chapter TWo}

Annex 1 Yield and detrended yield of selected districts ....................................................40

Annex 2 Paddy Yield (Kg/Ha) across 428 districts in Indonesia during 1990-2013..40

Annex 3 Self-calibrated Palmer Drought Severity Index (PDSI) gridded area ...............41

Annex 4 Average PDSI value in planting season (Sept-Dec) period 1990-2013 ...........41

Annex 5 Palmer Drought Severity Index classifications ....................................................42

Annex 6 Monthly variance explained for Sept-Dec planting season for rice in Indonesia

Annex 7 Fixed bandwidth method illustration in selected districts in Indonesia ........43

Annex 8 Fixed bandwidth method illustration in selected districts in Sumatera .........43

Annex 9 Spatial correlation between paddy yield and Indian Ocean Dipole (DMI) August at districts that are pseudo-significant at 5\% significance level ...........................44

Annex 10 Spatial Correlation between Paddy Yield and El Niño (ENSO) August at Districts that are Pseudo-significant at 5\% Significance Level............................................44

Annex 11 WGR panel results from ArcGIS............................................................................45

Annex 12 Table parameters of Weather Index Insurance (WII) using drought indices PDSI for rice production in major islands in Indonesia.......................................................4

Annex 13 GWR estimation using yield and time trend....................................................45

Annex 14 Parameters GWR panel for districts that are positively pseudo-significant at $5 \%$ significance level 46

Annex 15 Revenue without and with insurance for districts that are positively pseudosignificant at $5 \%$ significance level. 54

Annex 16 Summary report on regression estimates using different bandwidth and GWR models 61

Annex 17 Spatial Distribution of t-statistic Results 63 


\section{Annexes Chapter THREE}

Annex 1 Agro-climatic Oldeman 1901 - 1950, 1950 - 1980 and 1980 - 2015 ............99

Annex 2 Impact of Weather Shocks on Indonesia (1990 - 2017) .....................................99

Annex 3 Total Monetary Damages and Number of People Affected or Killed due to

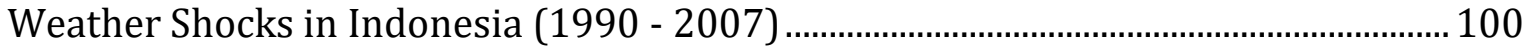

Annex 4 Drought events in selected areas based on author's drought method ........ 100

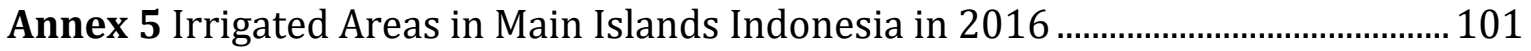

Annex 6 Statistical checks on the total income model specifications using graphs. 101

Annex 7 Scatter plots fitted and observed outcome variables 102

\section{Annexes Chapter FOUR}

Annex 1 Formal and informal insurance on outpatient expenditures' impact .......... 133 Annex 2 Mitigating characteristics as coping strategies to reduce bedrest impact. 134 Annex 3 Mitigating characteristics as coping strategies to reduce the impact on outpatient visit

Annex 4 Distribution of wet months and dry months 136

Annex 5 Distribution of outcome variables using tobit regression 136

Annex 6 Distribution of outcome variables using ordered probit regression 137 


\section{ACKNOWLEDGEMENTS}

All praises to the Almighty God for all the trials, strengths and His blessings in completing this thesis. I am very grateful to all of those who have helped and supported me go through the highs and lows of my Ph.D. journey. Importantly, this work would not have been possible without the financial support of the Economics of Disasters Doctoral Scholarship of which the sponsorship is provided by Victoria University of Wellington, the New Zealand's Earthquake Commission and the Ministry for Primary Industries.

I am exclusively thankful to my primary supervisor Professor Ilan Noy, the Chair in the Economics of Disasters of Victoria University of Wellington, for the continuous guidance to my Ph.D. work with patience, critical inputs, encouragement, and immense knowledge. I feel very lucky to have a supervisor who has been always supportive of my work, and who responded to my questions and queries so promptly. Many thanks also to my secondary supervisor AProf Bethanna Jackson of the School of Geography, Environment and Earth Sciences for her invaluable inputs, especially in the refinements of academic writing and discussion from another angle than merely from economics.

I wish to thank my fellow doctoral students, especially in the economics of disaster and hydrology group, for warm friendships and for sharing insightful opinions, know-how, and helpful hands. My sincere gratitude certainly goes to the administration team, teaching staffs and management of the School of Economics and Finance for the great support in the last 4 years. I also very much appreciate sustaining support from the Victoria Business School and Faculty of Graduate Research for their useful seminars, talks, courses and funding that helps further improve network and research skill set. I thank beforehand the thesis committee and examiners who are willing to offer time and greatly appreciated contribution to this work.

Finally, I heartily devote this work to my family who has given endless love and supports enduring this ups and downs pursuit; especially to Ibu, my mother, who has gone to heaven reunited with my loving father before the completion of this work. I offer all credits of this work to my beloved and fully supportive wife, Amelia, who always believes in me and raises me up even when I am down and in my most under-confidence state. The last word is dedicated to my three wonderful children Alifta, Bilal and Chiara, who always give me cheerful motivation after countless long nights and sleepless days. 


\section{ABSTRACT}

The weather-economy nexus has long had close attention from scholars and policy makers as weather hazards often have a significant impact on socioeconomic outcomes of populations around the world. A continuous understanding of this relationship is vital for societies to deal well with weather risk. This is particularly important in relation to climate change, which is likely to worsen the consequences of extreme weather as their frequencies and intensities increase. This thesis consists of three essays that demonstrate the adverse effects of extreme weather episodes on the local economy, using publicly available weather data and economic data sources. The essays use Indonesia setting as a case study, but the findings are likely to also be relevant for the situation in other developing countries located in the tropics that face similar socioeconomic challenges dealing with weather risk. The first essay, having identified the robust link between drought and variations of agricultural yield in the last decades, assesses the viability of weather-index insurance (WII) scheme against drought risk for rice farmers. The results suggest WII can play a cost-effective risk reduction role in drought sensitive regions, such as Sulawesi. The timing of drought as an exogenous shock to household economic outcomes is important as indicated in the results of the second essay. The estimated adverse effects to household monthly incomes and expenditures begin in the following year the drought occurs. Besides affecting economic outcomes, extreme weather also drives variations in health-related outcomes of adult individuals. This is empirically identified in the third essay that finds a robust connection between extreme rainfall and health outcomes. The results confirm earlier findings that people face higher probabilities of being affected by diseases during adverse events, especially during dry months. Estimations on coping mechanisms suggest heterogeneous effects with respect to, for example, the role of insurance. Lastly, the third essay finds that extreme weather episodes are negatively associated with non-quantifiable subjective well-being and life satisfaction. 


\section{Chapter ONE}

\section{INTRODUCTION}

Anomaly conditions of weather and its long average, climate, are associated with adverse changes in social and economic outcomes. Empirical findings suggest that the extremes in weather realizations, among others, affect agricultural yield, reduce consumption, incite social conflicts, and impact health outcomes. In low to middle-income countries, especially those that are in the tropics, weather-related disasters such as floods, droughts, and storms can cause significant losses and long-lasting disproportionate effects. The lack of infrastructure, disorganized spatial planning, and absence of financial risk transfer tools including social safety nets are typical problems in the developing world, along with high dependency on the primary industries that rely heavily on nature such as the agriculture sector. These dependencies amplify the socioeconomic impacts of extreme weather events. While a good understanding of the weather/climate-economy nexus is important for managing disaster risk, research progress in developing countries is often challenged by the limited availability and accessibility of data on historical weather and consistently observed economic indicators. This condition motivates empirical works in this thesis that overall examine the consequences of extreme weather conditions in the local economy and health outcomes across time, including a study on a potentially viable disaster risk reduction tool through a weather-index insurance scheme.

The thesis consists of 3 (three) essays, which are respectively elaborated in Chapters 2 , 3 , and 4. The essays use Indonesia as a case study for a number of reasons. Firstly, Indonesia is an interesting case of a tropical developing country with the majority of its population dependent on agricultural livelihoods that are susceptible to weather risk. Understanding weather dynamics is important for disaster risk reduction as the archipelagic nation has a complex variation of local weathers and climates due to its wide geographical areas and influence from global climate (i.e. El Nino Southern Oscillation). 
Thus, findings in the three essays may be also relevant for situations in other countries that share similar geographical features and socioeconomic characteristics with Indonesia. Secondly, research in this field needs considerable data requirements. There are publicly available high quality economic and weather data about Indonesia that cover several decades of observations. Sources for historical weather data include widely accessible gridded data from the United States' National Oceanic and Atmospheric Administration (NOOA) and the Central Research Unit of the University of East Anglia, United Kingdom. The economic indicators are gathered from various sources, for example, agricultural statistics from the Indonesia Ministry of Agriculture and household/individual data from the Indonesian Family Life Survey (IFLS). The combination of spatially specific weather data over time and ongoing longitudinal agricultural data or household survey IFLS enables long-term analysis of the variations of weather-economy relationships using panel fixed-effect specifications. Finally, this academic work is an attempt to advance the existing literature, especially in understanding the relationship between extreme weather and socioeconomic outcomes in developing countries. Relevant articles about this topic in Indonesia are still very limited.

In Chapter 2 (two), the first essay assesses the viability of weather index-based insurance to reduce drought risk for rice sector in Indonesia. The study is motivated by the fact that while farmers are prone to droughts, formal risk transfer tools, such as agricultural insurance, are lacking in Indonesia. Current insurance systems are afflicted by classical problems in conventional claim-based insurance type, like moral hazards, asymmetric information, and high transaction costs. The use of index-based insurance to protect farmers from catastrophic climatic risk, although increasing, is not without challenges. Among other obstacles in index-based schemes, basis risk is the main concern for a successful adoption. This essay offers a novel way to reduce basis risk by constructing district specific underlying indices based on establishing robust relationships with agricultural yield, which is modelled using Panel Geographically Weighted Regressions.

The third Chapter elaborates on the themes from the second essay. The research focuses on quantifying the effects of droughts that occurred recurrently in the period of observation 1990 - 2015. Drought potentially disrupts economic activities, especially in a population whose livelihoods are sensitive to weather outcomes. Here, the study 
measures the impacts of drought on several household incomes and expenditure using panel fixed-effect specification. There are new features introduced in this study to the existing literature, including the construction of periodic drought events using the Standardized Precipitation Evapotranspiration Index (SPEI) at the sub district level that is associated longitudinally with household economic outcomes and the importance of drought timing. The findings suggest that it is the drought that occurred last year had a significant impact on household incomes (drop between $7 \%$ and $44 \%$ ) and expenditures (increases between 1\% - 9\%).

The third essay (Chapter 4) looks at the health impacts on adult individuals who experienced extreme weather in the last 3 months. Here, the empirical analysis relates extreme weather episodes, which are identified based on excessive and shortage of rainfall in a month, to individual spatially-specific (sub-district), and various healthrelated outcomes. An additional investigation of life satisfaction impact, as a subjective measure of well-being, becomes one of the contributions to the literature. Model specifications rely on a panel fixed-effect approach that also controls time fixed-effects. The regression results suggest that dry month conditions have more potential health impacts than extreme wet month periods. For example, an adult individual who experienced 3 dry months in a row (rainfall below historic $10^{\text {th }}$ percentile) would have a higher probability (between 1 to 1.4 percentage point) to suffer from mild illness, digestion problems and difficult breathing than one who did not have that experience. 


\title{
Chapter TWo
}

\section{A VIABLE AND COST-EFFECTIVE WEATHER INDEX INSURANCE FOR RICE IN INDONESIA}

\begin{abstract}
:
The potentially adverse effects of droughts on agricultural output are obvious. Currently, Indonesian rice farmers have little financial protection from climate risk via catastrophic weather-risk transfer tools. Done well, a weather index insurance (WII) program not only provides resources that enable recovery, but also facilitates the adoption of prevention and adaptation measures and incentivises risk reduction. However, implementations of WII programs have faced difficulties because of basis risk (insurance payout differs from actual loss) - among several other obstacles. Here, we quantify the applicability, viability, and likely cost of introducing a WII for droughts for rice production in Indonesia. To reduce basis risk, we construct district specific indices that are based on the estimation of Panel Geographically Weighted Regressions models. With these spatial models, and detailed district level data on past agricultural productivity and weather conditions, we identify an algorithm that can generate an effective and actuarially sound WII in some districts but not in others. We then measure its effectiveness in reducing income volatility for farmers by reducing this basis risk, at the district level. We end by calculating an actuarially-robust and welfare-enhancing price for this scheme and prioritise the districts in which it can be implemented.
\end{abstract}




\subsection{Introduction}

The potentially adverse effects of droughts on agricultural output are obvious. In many high-income countries, the agricultural sector is at least partially protected, but in middle- and low-income countries, where markets and instruments for transferring catastrophic weather risk are almost always lacking, such protection rarely exists. This lack of financial risk transfer instruments means that losses associated with weather events are greater and economic recovery from them is more prolonged (FA0, 2015). In many countries, recurring weather shocks can induce serious adverse consequences to large numbers of people when agriculture provides the main livelihood for a large proportion of the population. With climate change, these problems are only likely to be exacerbated.

While there is no single solution for managing weather-hazard risks, there is robust evidence that suggests that insurance and other risk-transfer instruments can not only provide resources that enable recovery and increase resilience but also facilitate the adoption of prevention and adaptation measures, hence incentivising the reduction of catastrophic risk (UNFCCC, 2008; IPCC, 2012). Yet, in spite of the clear potential for significant risk transfer that is embedded in financial instruments such as insurance, these are still sorely lacking in most middle- and low-income countries.

Indemnity insurance is plagued by problems of moral hazard, adverse selection, and high transaction costs (Khalil et al., 2007; Rao et al., 2015). Given these difficulties in the provision of 'standard' indemnity insurance, weather-index insurance (WII) may provide an alternative to conventional insurance that can overcome many of the obstacles facing more conventional programs (Noy et al., 2017). Increasingly, WII is considered a mainstay of the menu of available risk transfer tools that may assist development as it transfers risk away from the poor and the vulnerable. Consequently, WII schemes have been trialled in several countries; e.g. the CADENA program in Mexico (Janvry et al., 2016). Disappointingly, their track record is less than stellar, with frequent lack of demand for these products or a reluctance to commit to these products beyond the first year (if no pay-outs were received). One hypothesis about these failures is that basis risk renders many of these schemes unattractive to farmers. At the extreme end, an insurance 
product with significant basis risk is more akin to a lottery ticket than to a risk-transfer tool.

In this paper, we examine the applicability, viability, and likely cost of introducing a WII for droughts for rice production in Indonesia. Given the size of the sector in Indonesia, and the general importance of rice as a staple source of calories in the region, a viable and cost-effective WII scheme for rice can provide tangible benefits to a large number of people and is clearly worth pursuing. Our findings show that WII could help reduce farmer's revenue fluctuation during drought period by up to almost $24 \%$.

A drought index insurance has been proposed in several countries in Sub-Saharan Africa, South Asia, and North Asia to cover farmers' losses from weather extremes (Skees et al., 2001; Rao et al., 2015; Tadesse et al., 2015). These initiatives have yet to make significant inroads in implementation in any developing country. There are multiple barriers to implementation, and these include failures in the insurance products that are being created, market failures in insurance markets caused by asymmetric information problems, and in the 'predictable a-rationality' of the insurers' intended customers. ${ }^{1}$ Here, we focus on only one problem-namely basis risk-and our work does not take into account all the other barriers to successful implementation of WII in Indonesia. In short, the purpose of this paper is to design a WII scheme that minimises basis risk and test the scheme's ability to successfully transfer drought risk for rice farming in Indonesia.

Basis risk is the possibility that the weather index used for triggering insurance payments does not sufficiently correlate with actual damage. This can lead to both type I and type II errors. ${ }^{2}$ Our aim here is to minimise basis risk, at the district level, by constructing district specific indices that are based on spatial modeling and the estimation of Geographically Weighted Panel Regressions (GWPR) models. With these tools, and

\footnotetext{
${ }^{1}$ By 'predictable a-rationality' we mean the well-documented ways in which people deviate from the rational homo economicus predictions that are used in traditional modelling in economics. The term, as used here, is a modified version of Dan Ariely's bestseller, Predictably Irrational. A comprehensive description of these predictable a-rationalities as they pertain the risk management is available in Kunreuther and Meyer's book The Ostrich Paradox.

2 Type I error refers to circumstances where payouts are given when no crop failure occurred and type II refers to situations where farmers endure crop losses without receiving insurance payouts.
} 
detailed district level data on past agricultural productivity and weather conditions, we construct an algorithm that generates an effective and actuarially sound WII.

As earlier suggested by Cai et al. (2014) in a different context, the GWPR approach provides us with an improved ability to associate weather dynamics with crop productivity in topographically and climatically diverse study areas. Once we have identified districts for which WII might be viable, we use Standard Deviation and MeanSemi variance efficiency tests, as in Shi and Jiang (2016), to show that the WII scheme we designed can reduce revenue risks from yield fluctuation due to drought conditions. We finish by quantifying this risk transfer benefit per district and thus provide an actuarially fair price for this product for each district.

This empirical exploration uses a panel dataset consisting of data about paddy production in 428 Indonesian districts, from the Ministry of Agriculture, annually for the period 1990-2013, and climate data from the United States' National Oceanic and Atmospheric Administration for 1950-2015. We show that the monthly Palmer Drought Severity Index (PDSI) can be used as the main component of the index as it reflects information on both rainfall and air temperature; the PDSI is generally considered a more sensitive and less noisy indicator of drought conditions (Dai et al., 2004). In our modelling, we also include anomalies of sea surface temperatures in the Pacific Ocean (the El Niño - Southern Oscillation ENSO phenomenon), and the Indian Ocean (the Dipole Mode Index DMI) (Naylor et al., 2001; Naylor et al., 2007; D'Arrigo \& Wilson, 2008; Iizumi et al., 2014). These additional explanatory variables allow us to obtain a more robust estimate of the relationship between climate indices and crop yield in Indonesian districts in order to identify the regions in which such an insurance index may be viable.

\subsection{Background and motivation}

Insurance markets are rife with market imperfections and failures that make insurance products very difficult to introduce in places where the institutions to overcome these imperfections are lacking. Market failures for conventional insurance include adverse selection, imperfect or asymmetric information, costly verification, moral hazard and the high cost of dealing with correlated risks. Index insurance is an alternative scheme using an observable index to trigger and quantify insurance payments (Skees et al., 1997; Skees 
et al., 1999; Kellner \& Musshoff, 2011; Miranda \& Farrin, 2012; Carter et al., 2017). Unlike conventional insurance that pays damages based on individual assessment of losses incurred, payment in index-based insurance is triggered based on an agreed underlying index and a pre-specified threshold, such as a weather parameter (e.g. rainfall or temperature). As such, for WII moral hazard is minimized, and if the index is based on a publicly observed parameter, there are also no information asymmetries (that plague indemnity-based insurance arrangements). Furthermore, as an underlying index is easy to monitor and verify, the verification costs are significantly lower per policy, and enforcement of these contracts is also significantly simpler.

\subsubsection{Weather Index Insurance: Empirical Research}

Studies of weather index insurance can be found in the early literature of Skees et al. (1999), Miranda and Vedenov (2001) and (Mahul, 2001). More recent works introduced new types of WII contracts such as composite index arrangements in China (Shi \& Jiang, 2016), and flexible contracts in Kazakhstan (Conradt et al., 2015). Alternatives to calculate actuarially appropriate commercial pricing of the insurance products are described in Clarke et al. (2012), while some projects focus on a specific peril like drought (Rao et al., 2015; Choudhury et al., 2016), extreme rainfall (Nieto et al., 2010), and high temperature (Spicka \& Hnilica, 2013).

Evaluation of a program post-implementation is much less common. An exception, Janvry et al. (2016) examine data from the CADENA crop insurance program in Mexico. They use a regression discontinuity approach and observations about specific insured events to conclude that while the costs of the program seem high, the farmers for whom insurance payments were triggered were later able to cultivate more land (in the following year). They conclude that under some weather risk scenarios, the program's benefits can outweigh its costs. Similarly, Bertram-Huemmer and Kraehnert (2018) evaluated the Mongolian livestock index insurance scheme after payments were triggered for policyholders during the 2009-2010 winter. They also observe better outcomes for those households that purchased insurance, even after accounting for the selection bias in the decision to purchase insurance. Without long-term data on risk and outcomes, however, these observations do not confirm the programs are cost effective. We take a different approach in this paper, as we design a hypothetical WII scheme for specific districts we 
identify as suitable. We then estimate how this hypothetical program would have performed, for insured households, had it been in place during the last several historical decades (i.e., the weather record in these decades).

Several reviews of the weather index insurance (WII) literature are available. These include Leblois and Quirion (2013), Tadesse et al. (2015), and Carter et al. (2017). Binswanger-Mkhize (2012) reviews the state of WII initiatives and argues that while products can potentially have beneficial welfare impact on small farmers who are otherwise not able to adopt other methods for transferring financial risk, these same farmers will find it difficult to afford their participation in these programs. Perhaps as a consequence of this, Binswanger-Mkhize (2012) observes that these schemes generally have very low take-up rates among their intended farmer/clients. He also argues that the presence of basis risk in WII programs is potentially too significant a problem for it to be worthwhile for governments to finance these programs. He suggests that other social safety nets, like employment generation programs, can have impacts that are more meaningful to the poor in dealing with the presence of adverse weather events.

Because of the possible link between basis risk and the low uptake of many WII schemes, some quantitative assessments have suggested ways to reduce basis risk. Carter et al. (2017), for example, examines this and recommend pursuing more technological and actuarial innovations that reduce basis risk. Others suggest using risk layering, by combining WII with other financial tools such as credit (Tadesse et al., 2015), enhancing strategic government supports (Skees et al., 2004; Janvry et al., 2016) and combining WII insurance with informal risk pooling to reduce basis risk and improve the quality of insurance coverage (Dercon et al., 2014). Another idea, that has not yet been explored as far as we know, and is also not yet explored in our work, is combining WII with more traditional indemnity clauses, with the aim of reducing the basis risk which plagues WII schemes.

The geographical location of the insured area along with the timing of observations of the index obviously influence the estimation of basis risk, as the underlying weather indices are spatially and temporally differentiated. In addressing these spatial and temporal variabilities, researchers have proposed different strategies. Khalil et al. (2007) consider an insurance contract using a spatially uniform climate index constructed based on the El 
Niño (ENSO) deviations for a regional rainfall index for mitigating flood risks in Peru. Paulson et al. (2010) apply spatial analysis techniques to address the absence of highquality data for weather variables - a typical problem in developing countries. Using interpolation methods, they create rainfall histories from a sparse grid of historical data and demonstrate the effectiveness of using that rainfall-interpolated data in designing insurance policies. Heimfarth and Musshoff (2011) evaluate spatial basis risk using a decorrelation function - a method that incorporates spatial dependence of weather patterns in analysing the correlation of underlying weather index and crop losses at different weather stations; with the aim of developing a WII scheme at the community level in the North China Plains.

Since our study area is large, encompassing a wide variety of environmental and climatological characteristics, it is reasonable to expect that the influence of weather on crop growth will vary across different locations. Earlier research has already established the variability of the link between weather and agricultural productivity using various methods such as explicit crop modelling or a statistical approach. For example, Ceglar et al. (2016) analysed the impact of temperature, solar radiation and rainfall variability on wheat and maize yields over 92 French administrative regions. They find notable spatial differences in the effect of the meteorological indices they use on crop yield.

\subsubsection{Spatial Regression Methods}

Even though earlier studies have found heterogeneity in the link between weather and crop yield across different study areas, most researchers still estimate 'global' regression parameters (by global we mean parameters that apply to the whole geographical area under investigation). Such global estimates can be inaccurate in understanding the correlation between the variables of interest and can, therefore, lead to imperfect calibrations of the proposed index and consequently basis risk. We, therefore, use methods that have been developed to produce localised versions of the traditionally global multivariate estimates, in order to reduce basis risk in the ultimate insurance product we design.

Spatial techniques are more common in ecology, geography, and epidemiology, but are increasingly being used in economics and associated disciplines, thanks to the increased availability of geo-referenced economic data and improved Geographic Information 
Systems (GIS) software. Several recent papers, in diverse areas of economics, have argued for the importance of spatial estimations (Anselin, 2001; Kelejian \& Prucha, 2010; LeSage \& Dominguez, 2012; Woodard et al., 2016). Specifically, this paper focuses on Geographically Weighted Regressions (GWR) as described in earlier papers (Brunsdon et al., 1996; Fotheringham et al., 1998; Brunsdon et al., 2002; Yu, 2010; Cai et al., 2014). GWR allows for the estimation of 'local' regression parameters while still accounting for the spatial distribution of the data by including geographically proximate observations, appropriately weighted (Fotheringham et al., 2002).

GWR is a methodology that incorporates both spatial heterogeneity and spatial dependence in the observed variables, allowing different relationships to exist at different points within a pre-determined radius around an observation (Brunsdon et al., 1996; Fotheringham et al., 1998; Brunsdon et al., 2002; Fotheringham et al., 2017). GWR weighs nearby observations more than distant observations using a distance decay function. Earlier works of GWR used cross-sectional datasets, but more recently, some researchers have been using panel datasets as well. Recent examples of papers using geographically-weighted panel regression (GWPR) techniques are Yu (2010) that studied urbanization and regional development in China and Bruna and Yu (2013) that investigated a regional wage equation model in Europe. For our purpose, the most relevant GWPR work is Cai et al. (2014) that analysed the spatially varying association between weather condition and corn yield across 958 U.S. counties from 2002 to 2006. No work that we are aware of has applied the GWR or GWPR approaches in investigating a WII scheme.

\subsubsection{The Study Area: Indonesian Districts}

Indonesia is an archipelagic country extending 5,120 km from east to west and 1,760 km from north to south and consisting of about 6000 inhabited islands. Major ones are Sumatra, Java, Kalimantan, Sulawesi, and Papua and the main archipelagos include BaliNusa Tenggara and the Maluku. Indonesia has 514 administrative districts (98 cities and 416 regencies - as of 2016). Rich volcanic deposits have endowed many of the Indonesian islands with very fertile soils providing ideal conditions for intensive rice agriculture.

Because of its proximity to the equator, the climate in Indonesia is very stable year-round, with temperatures averaging between $23-25^{\circ} \mathrm{C}$ in mountain areas and $28-30^{\circ} \mathrm{C}$ in the 
coastal plains. The country has only two seasons: wet and dry, though the difference in rainfall between the seasons varies across the regions. For example, Kalimantan and Sumatra experience only slight differences between the seasons (2000 - 3000mm annual rainfall), whereas Nusa Tenggara experiences far more pronounced variance in rainfall (less than 2000mm annual rainfall). Indonesia has abundant rainfall, particularly in west Sumatra, northwest Kalimantan, west Java, and western Papua, though the islands closest to Australia are drier. Monsoons usually blow from the northwest in November to March and from the south then east in June to October. In general, Indonesia has two growing seasons for rice - though, in regions where irrigation networks are available like in Java and Bali, rice may be cultivated in three annual cycles. The main growing season starts after the onset of the monsoon, which generally occurs between September and December. Harvest can be as early as January or as late as April, given this uncertainty in the monsoon arrival. The secondary planting season usually begins in April and is harvested in September.

The agriculture sector is an important mainstay for the Indonesian economy, providing jobs for more than 40 million people (about 38\% of the total labour force), and contributing around 15\% of GDP in recent years. In rural areas where almost half of Indonesians live, farming (including livestock and fisheries) is the main source of income for $63 \%$ of households. Indonesia has a total area of 191 million ha. About 57 million ha of this land is cultivated with cash crops (palm oil, cocoa, rubber, coffee, spices and tea), food crops (rice, maize and cassava), horticulture (tomato, carrot, banana, mango, mangosteen, chrysanthemum, rose, etc.), and livestock (chicken, duck, goat and cattle). The total land area of sawah (a wetland for rice cultivation accounting for about $80 \%$ of rice grown in Indonesia) is 8.1 million ha. Almost half of sawah cultivation is located in five major producing provinces: East Java, West Java, Central Java, South Sulawesi, and South Sumatra.

Rice production in Indonesia is the third largest in the world, after China and India, but like these two bigger countries, most of it is consumed domestically. Rice is the main staple food and its production involves 14 million small farmers having on average less than half a hectare of land per household to cultivate. Paddy yield in Indonesia depends greatly on rainfall, as irrigation infrastructure only exists in limited areas. About $17 \%$ of the cultivated area is irrigated, mostly in Java, and only $10 \%$ of this area is irrigated 
effectively (IFC, 2012). This implies that a large majority of rice farmers are susceptible to drought risks.

\subsubsection{About WII in Indonesia}

Agricultural Insurance (AI) in Indonesia is still very limited, even when compared to other countries at a similar stage of industrialization (Mahul \& Stutley, 2010). Indonesia officially started an insurance program less than 10 years ago through several government-funded pilot projects. Conventional indemnity AI that offers protection for multiple perils-flood, drought, and pest and disease-is the most commonly used approach being tested. Evaluation of several smaller pilot programs found these schemes to be unfeasible due to the inherent problems of conventional indemnity programs discussed above (asymmetric information, moral hazard, and high transaction costs). Therefore, alternative modalities such as index based insurance are needed to resolve impediments to program implementation (Insyafiah and Wardhani, 2014). Recently, the Government of Indonesia launched the $3^{\text {rd }}$ Economic Package policy in October 2015; the program includes a nationwide subsidised AI scheme for the rice sector. As this program is being trialled for small-scale rice farmers, the government is also modelling alternative types of AI for fishermen and cattle farmers including the possible use of index insurance.

Literature about WII in Indonesia originated in Estiningtyas et al. (2011), a project that assessed the feasibility of a WII for rice production in three villages in Indramayu district using a rainfall index. It concludes that WII can cost-effectively help farmers manage the risks during long drought periods. IFC (2012) explored the feasibility of WII for drought for maize production risk in Eastern Indonesia by testing it in two districts. The study found that it is "technically feasible", and that there is a readily identifiable business model to support WII for maize production in the studied areas. Separate research by Kawanishi and Mimura (2015) investigated the feasibility of weather index insurance in the Bengawan Solo River basin and tested it in two districts. They used the correlation coefficients of monthly rice harvest failure and monthly rainfall but found a significant basis risk problem. They concluded that in that region, a WII scheme may not be an effective tool to manage weather risk. No research has tried to conduct a more comprehensive feasibility study, i.e. one moving beyond considering only a few districts in a very geographically narrowly-defined region. Without such a study, any doubts about the external validity of a WII program in Indonesia will be very difficult to dispel. 


\subsection{Research questions, data, and methods}

Four sequential questions of interest guide our research methodology: 1) How can we exploit the spatio-temporal variation in the relationships between weather indices and rice paddy yield to understand better the effect of weather variability during planting season on crop productivity at the district level in Indonesia? 2) Which districts show sufficiently robust links between drought indices and rice yields? 3) Can we develop, using the spatially varying relationships between weather indices and crop yield, a WII program for the Indonesian rice sector in the districts previously identified? 4) Does this WII scheme reduce the variability of income in the 'insurable' districts?

It is important to emphasize that while we focus on rice farming in Indonesia, we view our methodological contribution as wider. The overall aim of this research is to introduce a novel way (geographically weighted panel regression) to develop an algorithm for the construction of viable agricultural index insurance. This same algorithm can be applied elsewhere, and on different crops, as long as the empirical association between the weather index and crop production can be established spatially.

\subsubsection{Crop Data}

We obtained agricultural statistics from the Indonesian Ministry of Agriculture comprising annual data of the harvested area, productivity or yield, and paddy production at district level within the period of 1990 - 2013. For regression analysis, we use yield data in 428 districts, as we are limited to districts with consistent and complete information on rice production as well as the availability of geographical data for GIS analysis. The figures come from the survey of the regional offices of the Ministry of Agriculture that regularly collects data of harvested areas every month. Paddy productivity or yield data are collected every season (4 months cycle) using a specific statistical survey on $2.5 \times 2.5$ meter squared plots by the local offices of the Centre of Statistical Bureau (BPS) and the agricultural offices.

To recognize spatial variability, we calculate the average paddy yield-per planting season in the period of 1990 - 2013 across the observed districts and map the data (See Annex 2). Districts in Java and some parts of Sulawesi and Sumatra dominate high paddy productivity while low productivity is prevalence across districts in Kalimantan and 
Papua. The low yield ranges from low 1.7 - 1.9 ton/ha in several districts in Central Kalimantan (Palangkaraya, Barito Utara, Gunung Mas, Lamandau) to high yield of 5.8 - 6 ton/ha in several districts in East Java (Malang, Pasuruan, Magetan). Later, we will see that it is mostly the districts with average productivity that exhibit the best potential for a WII scheme. Very high productivity districts are usually irrigated (and therefore less vulnerable to droughts), while very low productivity districts also have less variation in productivity that is associated with the drought conditions.

\subsubsection{Weather Data}

Weather related factors are important determinants for agricultural production. Here, we focus on the correlation between weather measures and crop yield during planting season. ${ }^{3}$ Since we are interested in the effect of drought on crop productivity, our main explanatory variable is the Palmer Drought Severity Index (PDSI) where we also include climate indices - namely the El Nino - Southern Oscillation (ENSO) index and the Indian Dipole Mode Index (DMI) as factors controlling for local weather conditions. While many studies have investigated the effects of local weather and climatic conditions on all stages of the rice growth cycle, a growing body of evidence also reports the impact of global climate patterns like ENSO and DMI on local weather variables, particularly affecting rainfall and temperature (and hence drought risk). Several studies have found a significant effect of global climate condition on rice cultivation in Indonesia (Naylor et al., 2001; Naylor et al., 2007; D'Arrigo \& Wilson, 2008; Rosenzweig et al., 2014) - See Annex 6 for an example. We obtained weather data from the United States' National Oceanic \& Atmospheric Administration (NOAA).

The PDSI was firstly developed by Palmer (1965) to measure the cumulative deficit in atmospheric water balance using information on precipitation, temperature, and altitude in US regions. The latter version of the PDSI has been used globally for monitoring and forecasting drought incidents, analysing the impact of climate variations, and also for index insurance (Skees et al., 2001; Dai et al., 2004; D'Arrigo \& Wilson, 2008; Wu et al., 2015). For this research, we use the monthly mean calibrated Dai's PDSI gridded dataset, which is updated until December 2014 and available online at the US Earth System

\footnotetext{
${ }^{3}$ Compared to flood or storm surges, drought is the most dominant climatic hazard that results in considerable losses to the agriculture sector in Indonesia in the last 4 decades (Lassa, 2012).
} 
Research Laboratory website. ${ }^{4}$ Dai et al. (2004) first introduced this monthly dataset of PDSI from 1870 to 2002 using historical precipitation and temperature data for global land areas on a $2.5^{\circ}$ grid on both latitude and longitude.

Table 1 Summary Statistics Data (428 districts in 24 years)

\begin{tabular}{lcccc}
\hline & Mean & SD & Min & Max \\
\hline $\begin{array}{l}\text { Administrative } \\
\text { Area district (Ha) }\end{array}$ & 440,981 & 625,448 & 986 & $4,531,178$ \\
\hline Crop Data & & & & \\
Yield (Ton/Ha) & 4.28 & 1.07 & 0.40 & 8.40 \\
Indices & & & & \\
PDSI (-8 to 8 -- dry to wet) & -0.41 & 2.42 & -7.58 & 7.25 \\
ENSO $(\stackrel{\circ}{-}$ ) & 0.03 & 0.67 & -1.16 & 1.74 \\
DMI $(\stackrel{\circ}{-})$ & 0.26 & 0.39 & -0.46 & 1.10 \\
\hline
\end{tabular}

The current calibrated dataset accounts for the effects of radiation, humidity and wind speed in addition to rainfall and temperature. We assign each district a PDSI value per year in PDSI gridded area as shown in Annex 3 and Annex 4. It shows average PDSI value during planting season (September - December) 1990 - 2013. In Annex 5, we include a common PDSI classification that indicates the severity of a wet or dry spell which generally ranges from -6 to +6 , with negative values denoting dry spells and positive values indicating wet spells. Following D'Arrigo and Wilson (2008), we use PDSI values during the planting season (Sept-Dec) to detect drought incidence that affects crop yield in the following season.

The El Niño/Southern Oscillation is a global phenomenon that represents a recurring pattern of climate variability in the Equatorial Pacific. It signifies anomalies in both seasurface temperature and sea level pressure (Southern Oscillation) where the anomalies for warming period are referred to as El Niño and the cooling periods are referred to as La Niña. In this paper, we control for ENSO using the Oceanic Niño Index 3.4 - it measures the average of temperature anomalies over Central-Eastern Equatorial Pacific $\left(5^{\circ} \mathrm{S}-5^{\circ} \mathrm{N}\right.$, $\left.170^{\circ} \mathrm{W}-120^{\circ} \mathrm{W}\right)$. We use historical monthly data for ENSO since 1950 which is available online from the Climate Prediction Centre. ${ }^{5}$

\footnotetext{
${ }^{4}$ http://www.esrl.noaa.gov/psd/data/gridded/data.pdsi.html

5 http://www.cpc.ncep.noaa.gov/products/analysis_monitoring/ensostuff/ONI change.shtml
} 
The Indian Ocean Dipole Mode Index (DMI), measures irregular differences in the temperature between two areas in the Western and the Eastern Indian Ocean. Like the ENSO, it may relate to rice productivity in Indonesia because of its relationship with rainfall. A positive phase of DMI is indicated by lesser than average temperatures and greater precipitation in the Western Indian Ocean region, and with a corresponding cooling of waters in the Eastern Indian Ocean. This pattern tends to cause droughts in Indonesia and Australia. The negative phase of the DMI brings about the opposite conditions, with warmer water and greater precipitation in the Eastern Indian Ocean, and cooler and drier conditions in the Western part.

\subsubsection{The Spatial Models}

Our data is a panel of weather observations and paddy yield of 428 districts for a 24-year period (1990-2013). By using GWPR, we are able to utilize both the cross-sectional and time-series dimensions of the panel of observations we have, to account for both timeinvariant differences between districts (such as soil and climate conditions). In order to remove non-weather variable effects such as policy changes and technological improvement that might change over time, we detrended the paddy yield data using the Hodrick-Prescott filter (Baum, 2004). ${ }^{6}$ We present the deviation between detrended yield and actual yield in Annex 1. For comparison purposes, we also used original yield data in estimating the weather effect on yield variation. In doing so, we specify the model with time trends to control for the effects of other variables than weather (such as technology improvement). The estimation results using the original data are not identical with estimation using the detrended yield data but show similar patterns on the statistically significant areas (see Annex 13 for a visual overview, detail estimation results are available upon request).

\subsubsection{Fixed Effects, Spatial Auto-Regressive, and Spatial Error Models}

Fixed Effect modelling is the most common regression technique used to analyse the relationship between climatic change and crop yield (see Deschenes and Greenstone (2007) for an example). The model is specified as:

$$
y_{i t}=\beta_{0 i}+\sum_{k} \beta_{k} x_{i t k}+\varepsilon_{i t}
$$

\footnotetext{
${ }^{6}$ Previous work have shown that de-trending agricultural yield time-series data is useful for isolating the impact of technological changes on crop yield, particularly for actuarial purposes, see for example, Skees et al. (1997).
} 
where $i$ represents a district and $t$ indicates year; $y_{i t}$ denotes paddy yields for district $i$ at year $t ; x_{i t k}$ denotes the $k$ weather indices for district $i$ at year $t ; \beta_{k}$ is a weather-index coefficient that is constant across districts and across time; $\beta_{0 i}$ denotes time-invariant fixed effects; and $\varepsilon_{i t}$ is the error term. Previous research does not support the assumption that $\beta$ is indeed constant across districts, and we find that assumption unattractive if we are to develop a WII product that minimizes spatially-differentiated basis risk.

A second approach - spatial regression modelling - recognises the dependency between nearby observations in spatial data through the covariance structure of the error terms but still provides only global parameter estimates (Anselin, 1992; Anselin, 2001; LeSage \& Dominguez, 2012). These models consider spatial spill-overs in the dependent variable and specify the endogenous variable corresponding to a cross sectional unit in terms of a weighted average of variables corresponding to other cross sectional units, plus a disturbance term (Kelejian \& Prucha, 2010). Although these methods recognize and incorporate spatial dependency, they have limited use when the relationships between the variables of interest do vary over space ( $\beta$ is not uniform across districts). We, therefore, proceed with a Geographically Weighted Regression analysis that allows us to obtain district specific estimates for $\beta$ and thus investigate a potentially different WII scheme for each district.

\subsubsection{Geographically Weighted Regression (GWR)}

GWR uses neighbouring observations for estimation of a local regression at each point in space with a subsample of spatially-weighted data, weighted according to the proximity of each observation to each regression point. Consider a linear (global) regression model:

$$
y_{i}=\beta_{0}+\sum_{k} \beta_{k} x_{i k}+\varepsilon_{i}
$$

GWR extends this global regression by allowing local parameters for estimation:

$$
y_{i}=\beta_{i 0}+\sum_{k} \beta_{i k} \mathrm{x}_{i k}+\varepsilon_{i}
$$

where $y_{i}$ is the observation of the dependent variable at geographic location $i, \beta_{i k}$ is the coefficient parameter of the $k^{\text {th }}$ independent variable at geographic location $i . x_{i k}$ denotes the $k^{\text {th }}$ predictor variable at location $i$ and $\varepsilon_{i}$ denotes the error term. Although the GWR model (3) is a simple extension of the global linear model in (2), estimating the 
coefficients in model (3) is more difficult since there are not enough degrees of freedom. GWR assumes that coefficient parameters are not random, but rather that they are a deterministic functions of location in space (Fotheringham et al., 2002). To this end, a Weighted Least Square (WLS) method is used to calibrate regression model (3) with the assumption that observed data near to location $i$ have more of an influence in the estimation of $\beta_{i k}$ than do data located farther away (geographically). Ordinary Least Square (OLS) minimizes the sum of square residuals. In WLS, a weighting factor $\mathrm{W}$, is applied to each squared difference before minimizing the sum of square residuals, so that the deviations of some predictions incur more of a penalty than others. Estimation of parameters can then be written in the form:

$$
\hat{\beta}(i)=\left(X^{T} W(i) X\right)^{-1} X^{T} W(i) Y,
$$

where $\hat{\beta}$ is an estimate of $\beta, W(i)$ is an $n \times n$ spatial weighting matrix, its diagonal elements are zero and the diagonal elements are the geographical weighting of each of the $n$ observed data for regression point $i$. The weighting matrix is computed for each point $i$ according to a decay function based on the assumption that the observations nearer to the regression point are assigned more weight to represent their larger influence than observations further away and the weight decays linearly in distance.

The next step in estimating the GWR is choosing the bandwidth (the radius in which observations are still included for each regression). There are mainly two types of kernel functions used to determine the shape and extent of the bandwidth: Fixed and Adaptive. A circular neighbourhood of fixed (ad-hoc) radius is where each local regression analysis includes all observations within the fixed distance from the regression point, and the Adaptive algorithm is when a flexible algorithm is used to pre-determine a constant number of neighbouring sample points. Both types of functions employ the same principle of declining weights with distance. Equally important for the GWR is determining the distance to the regression point that will be used as it defines how much each observation will be weighted (Brunsdon et al., 2002; Yu, 2010).

In this paper, we specify a spatial weighting function using the fixed method of Gaussian kernel (Brunsdon et al., 2002; Fotheringham et al., 2002; Yu, 2010). For the bandwidth parameter, we select the optimal distance to derive bandwidth as generated by the GIS program. In exploring best results (more study areas that show statistically significant 
weather effects) and for comparison purposes, we also tested several regression estimates using different type of kernel and bandwidth methods (see Annex 16 for summary tests report).

Most GWR works use cross-sectional analysis, but new methods that exploit panel data using GWR can potentially give more accurate inference of model parameters and reveal new findings that might be hidden under the standard cross-sectional model described above. The first study to use GWPR was Yu (2010). Later on, Bruna and Yu (2013) investigated the effect of Market Potential on regional wages of European regions using GWPR. They showed how spatial change across Europe was particularly high for specific regions. Most recently, Cai et al. (2014) investigated the effect of weather on corn yield for 958 U.S. counties from 2002 to 2006 with a panel GWR and found that temperature tends to have negative effects on corn yields in warmer regions and positive effects in cooler regions.

In general, the GWPR model considers the earlier framework with an additional temporal component $(t)$ in each independent variables and error:

$$
y_{i t}=\beta_{i 0}+\sum_{k} \beta_{i k} x_{i k t}+\varepsilon_{i t}
$$

Compared to the Fixed Effects model equation (1), the panel GWR allows the vector of coefficients $\beta$ to vary across $i$ (district) but not across $t$ (time). We assume that bandwidths and the spatial weighting function are time-invariant, because spatial relationships among the districts do not change over time (especially in a short panel such as ours). Since the estimation will include both spatial and temporal observations, the matrix dimension becomes $n t \mathrm{x} n t$ and all observations used in each local model are weighted by the time invariant spatial weight function.

In order to convince the reader that the panel GWR should be our preferable model, we also estimated the other models described earlier, including estimation of fixed effects panel model and the standard spatial AR models with global coefficients. Accurate estimation-a good statistical fit-is important in this paper's context, as our aim in estimating these equations is to identify a model that will reduce spatially-sensitive basis risk when constructing a WII scheme. 


\subsubsection{Actuarial Analysis: Design and Valuation of index insurance}

As the GWR estimates a different model for each district, we can focus only on those districts for which the weather indices (in particular the PDSI) have a statistically significant predictive power for crop yield in the multi-variate model. Having thus identified the subset of districts for which it is feasible to design index insurance based on the indices we have, the next step is determining the optimal trigger and exit thresholds of the underlying weather index that will trigger the payment of compensation, and the cap on that compensation, respectively.

\subsubsection{Cluster Analysis for the Thresholds}

We apply a model-based clustering analysis to determine the optimal trigger and exit thresholds of the PDSI index for drought identification as introduced by Choudhury et al. (2016). Cluster analysis is a data analysis technique for organizing observed data into clusters, based on combinations of relevant factors. It classifies observations so that each object is very similar to each other within the cluster with respect to some criterion. In this paper, our underlying weather variable for the WII is the PDSI drought index; as we reported, it has a statistical association with paddy yield data. Therefore, we focus on these two variables in identifying index thresholds (trigger and exit) using cluster analysis. See the detailed discussion about model-based cluster analysis in Fraley and Raftery (2012).

As the more negative PDSI value spells drier conditions (see Annex 5), it is reasonable to assume that the lower the PDSI value the higher the probability of crop loss. With the cluster analysis tool, we can group observations of higher PDSI value (wet condition) based on similarity with higher yield observations and dry condition or lower PDSI value with lower paddy yield observations. The resulting clusters of observations should then exhibit high internal homogeneity and high external heterogeneity (Choudhury et al., 2016). In other words, these clusters are formed based on maximizing the similarity of observations within each cluster while also maximizing the difference between the clusters.

For comparison, we also run analyses to determine triggers of the WII using logistic regression models which are solved by Bayesian estimation (as in Khalil et al. (2007). 
With this method, we found the index thresholds are a bit lower than the index (trigger) resulting from the cluster analysis. ${ }^{7}$

\subsubsection{Pricing the Index Insurance}

Pricing an insurance contract should reflect the degree of the risk that is being insured. Consider the following standard cost equation of the commercial pricing insurance:

Price of Insurance $=$ Expected Annual Loss + Expense Loads + Risk Factor,

where Price of Insurance is the insurance premium, Expected Annual Loss (EAL) is the expected probability of annual loss - i.e., the average insurance claim payment that is paid out each year. Expense Loads considers all costs of covering administrative and operational expenses of providing insurance, such as costs for loss assessment, monitoring, administration, product delivery, and capital costs/profits. Risk Factor is an additional load to the premium usually charged by a risk-averse or risk neutral insurance company to protect it against the possibility that it is under-valuing the underwritten risk (Smith \& Watts, 2009). The expense loads and risk factor are typically assumed to be proportional to the present value of the EAL.

In an index insurance contract, there are significant transaction cost savings that can be transferred to the insured party in the form of a lower premium, compared to conventional indemnity-based insurance. Therefore, in calculating the price of WII, we exclude some components of expense loads such as the cost for controlling adverse selection (e.g. collection/surveys of farm-level information), cost for conducting loss assessment, and cost for controlling moral hazard (e.g. by monitoring preventive actions at the farm). We assume that administration cost is lower since WII is simple and uniform and contracts do not need to be tailored to each policyholder.

In this study, considering the proposed WII scheme is new and the targeted clientele is made up of small-scale rice farmers, it is reasonable to assume that the WII scheme will receive support from the government through load factors subsidy. ${ }^{8}$ Thus, here we only

\footnotetext{
${ }^{7}$ The greatest difference is found in Kalimantan. The trigger level for Kalimantan is almost one and a half points PDSI lower than the cluster analysis trigger (see Annex 12 for the detail results). This resulted in less coverage and a lower insurance premium.

8 In many cases, and for many reasons, private sector risk transfer mechanism are not available, and this justifies public sector investments in weather-related agricultural insurance (Mahul, 2001; Miranda \& Vedenov, 2001; Skees et al., 2004; Owen \& Noy, 2017)
} 
use actuarially fair premium or pure risk premium as the price of insurance assuming away the loading factors.

As we envision a government-supported program, we do not think it is politically feasible to price it differently in each district (even if we can evaluate the risk associated with each district separately). We, therefore, price the insurance product at the island or islandgroup (regional) level. As we have seen in the GWPR analysis, geographically-proximate districts are quite similar, so this choice does not imply we are pricing the risk very far away from the optimal pricing per district. On average, in each island-group, the pricing will be actuarially-fair, even if there are some deviations from that across the districts in that region.

The actuarially fair premium-henceforth 'premium'-is calculated as the expected value of the future payoffs of the insurance every year (equal to Expected Annual Loss) discounted by the annual risk-free interest rate $(r)$ for $t$ year contract. Consider:

$$
\text { Premium }=\text { Present Value }(\text { Average Payout })=\frac{1}{e^{r t}}(E A L) \text {, }
$$

In general, the pricing of the WII product is based on the underlying payment structure, should a payment be triggered by the index and the probability distribution that describes the possible observed valued of the index. The following equation expresses a payment (Choudhury et al., 2016):

$$
\text { Payout }=\left\{\begin{array}{cc}
I A & \text { if } P D S I_{A} \leq P D S I_{E} \\
I A\left(\frac{P D S I_{T}-P D S I_{A}}{\text { PDSI }_{T}-P D S I_{E}}\right) & \text { if } P D S I_{E}<P D S I_{A} \leq P D S I_{T} \\
0 & \text { if } P D S I_{A} \geq P D S I_{T}
\end{array}\right.
$$

where IA is the insured amount. We set the insured amount to equal the average cost of agricultural inputs (seed, fertilizer and pesticide). ${ }^{9}$ The insured amount is set as the cost of inputs rather than the actual experienced reduction in revenue (the market value of the crop loss) because a minimum aim of such an insurance program is to enable farmers to replant in the next season. Given the low demand for insurance products discussed in

\footnotetext{
${ }^{9}$ According to Statistics Indonesia, for wetland paddy in 2015, that amount is IDR 2 million. The full value of the average wetland paddy crop per hectare is IDR 17 million; https://www.bps.go.id/statictable/2015/09/25/1855/nilai-produksi-dan-biaya-produksi-per-musim-tanam-perhektar-budidaya-tanaman-padi-sawah-padi-ladang-jagung-dan-kedelai-2014.html.
} 
the introduction, and amply documented, we wanted to minimise the cost of the insurance product developed here. The cost of inputs is significantly lower than the loss of revenue. Farmers can, in principle, buy multiple contracts, if they desire and can afford the higher premiums. As such, one should view this decision to have $I A$ be based on inputs as the minimum (micro-insurance) contract available.

$P D S I_{A}$ denotes actual PDSI in planting season (Sept-Dec), $P D S I_{T}$ is a trigger, a PDSI threshold where a payout starts, $P D S I_{E}$ denotes exit threshold where the maximum payout = insured amount (IA) is paid.

The critical step in pricing WII is to estimate EAL (the average pay-out each year). We use historical data for the PDSI, as the underlying weather index, to simulate what the insurance cost would have been had the insurance product been in place in previous years. ${ }^{10}$ In this paper, the expected annual loss is calculated using normal probability and numerical integration as applied in Choudhury et al. (2016):

$$
\begin{gathered}
E A L=I A \int_{0}^{P D S I_{E}} f\left(P D S I_{E}\right) d_{P D S I_{E}}+\int_{P D S I_{E}}^{P D S I_{T}} I A\left(\frac{P D S I_{T}-P D S I_{A}}{P D S I_{T}-P D S I_{E}}\right) f\left(P D S I_{A}\right) d_{P D S I_{A}} \\
E A L=I A \int_{0}^{P D S I_{E}} f\left(P D S I_{E}\right) d_{P D S I_{E}}+\frac{I A}{P D S I_{T}-P D S I_{E}} \int_{P D S I_{E}}^{P D S I_{T}} P D S I_{T} f\left(P D S I_{A}\right) d_{P D S I_{A}} \\
-\frac{I A}{P D S I_{T}-P D S I_{E}} \int_{P D S I_{E}}^{P D S I_{T}} P D S I_{A} f\left(P D S I_{A}\right) d_{P D S I_{A}}
\end{gathered}
$$

Given normal mean and standard deviation, we then can calculate the probability of PDSI that exceeded thresholds $P D S I_{T}$ and $P D S I_{E}$ :

$$
\begin{gathered}
E A L=I A\left(F\left(P D S I_{E}\right)\right)+\frac{I A}{P D S I_{T}-P D S I_{E}}\left(P D S I_{T}\left(F\left(P D S I_{T}\right)-F\left(P D S I_{E}\right)\right)\right) \\
-\frac{I A}{P D S I_{T}-P D S I_{E}} \int_{P D S I_{E}}^{P D S I_{T}} P D S I_{A} f\left(P D S I_{A}\right) d_{P D S I_{A}}
\end{gathered}
$$

To solve the third terms, we apply numerical integration on the historical PDSI data to get an approximate value of PDSI actual $\left(P D S I_{A}\right)$ that falls between the trigger and exit thresholds.

\footnotetext{
${ }^{10}$ Historical loss cost data may not be adequate for estimating future indemnities if the insurance product covers losses from extreme but infrequent events which may or may not have occurred over the observed historical period.
} 


\subsubsection{Efficiency Test to Measure WII's Risk Reduction Capability}

Finally, we test the robustness of WII in reducing the underwritten risk using two different measures of the revenue of rice farming without and with WII - the standard deviation (SD) and the mean-semivariance (MSV). We analyse the reduction of variance $\left(\mathrm{SD}^{2}\right)$ and the increase in MSV during the 24 years observed if WII were to be retroactively be implemented. We can thus measure the effectiveness of the scheme in lessening rice income variability during drought periods. We implicitly assume the investment portfolio of the representative farmer in each district consists only of rice-production assets and the proposed WII contract, and they have no other risk transfer tools. ${ }^{11}$ A rice farmer who buys a WII product may expect to have lower returns on his/her investment portfolio, due to a loaded premium, but he/she may expect to have reduced risk. This is measured by the variance of the returns on the portfolio.

Firstly, we analyse the effectiveness of WII to hedge the risk by comparing the distribution of revenues of rice farming without WII and with WII at each district. We can measure it by looking at the standard deviation of revenue per hectare from paddy farming without insurance, $R_{0}$ - defined as: $R_{0}=p Q . Q$ denotes paddy yield $(\mathrm{kg} / \mathrm{ha})$, a function of the stochastic weather variable PDSI. $p$ is expected postharvest crop price $(\mathrm{Rp} / \mathrm{kg})$, which in this paper we set constant at Rp.4,000/kg. In the second scenario where a crop insurance is purchased, a farmer may get compensation of a pay-out $\left(F_{T}\right)$ if the underlying weather variable exceeds the trigger while he also has to pay the insurance premium $\left(F_{0}\right)$. The payout is a function of the underlying weather index $x$, thus the revenue per hectare from paddy farming with WII, $R_{1}$, becomes: $R_{1}=R_{0}+F_{T}(x)-$ $F_{0}$. We then calculate whether the difference between the variance of revenue with and without insurance is statistically significant.

Next, we calculate the mean-semi-variance (MSV) to assess how effective the WII is at reducing income shocks during drought periods. The difference from the SD measure is that MSV counts only observations below the expected value (i.e., downside risk). While variance (SD) can give insight about the extent of risk exposure of a portfolio including the up-side risk, MSV focuses on estimating the possible negative effect (loss) for a

\footnotetext{
11 The assumption that insurance is the only risk transfer tool available is further discussed in the conclusion.
} 
portfolio. Minimizing downside risk is of potential interest to policymakers in particular, and to risk-averse households, as most small-scale farmers are.

We start by calculating deviations of rice revenue below the average and ignore those observations above the mean. This semivariance between revenue of rice farming without WII and with WII represents the threat of loss. Consider the following:

$U_{i}=\left\{\begin{array}{ll}R_{i}-E(R) & \text { if } R_{i}<E(R) \\ 0 & \text { if } R_{i} \geq E(R)\end{array}, \quad\right.$ and $\quad \sigma_{\text {semi }}^{2}=\frac{1}{n} \sum_{i=1}^{n} U_{i}^{2}$,

where $U_{i}$ denotes the investor's utility, $R_{i}$ is farming revenue (calculated both with and without WII), $E(R)$ is the expected value of the revenue (without and with WII), $n$ denotes the total number of observations and $\sigma_{\text {semi }}^{2}$ denotes the semivariance of revenueyears that are below the expected (average) value. To this end, we apply MSV to measure the shortfall of rice revenue risk during drought weather condition by analysing the exposure level of revenue at-risk $\mathrm{V}$ that is relative to semivariance:

$$
V=E(R)-\frac{1}{2} k \sigma_{\text {semi }}^{2}
$$

where $k$ is the coefficient of relative risk aversion and $V$ denotes the revenue at-risk. Here we calculate the MSV for different $k=0.1,0.2$, and 0.3 (Eeckhoudt $\&$ Gollier, 1995). ${ }^{12}$

\subsection{Result: spatially varying relationship of weather and paddy yield}

For the cross sectional GWR model regression, results indicate very few districts that are positively pseudo-significant at 5\% significance level. ${ }^{13}$ Since general climatic conditions are very similar across provinces, and the important variation we are interested in is in the weather variability during the rice growing seasons, this absence of differentiation between results arising the cross-sectional estimation may not be surprising. The panel estimation results are shown in Table 2 . We start with the three regression models that assume global (constant) coefficients across districts (in columns 1-3). We estimate the model with fixed effects, and with two spatial models. In all three, we find robust

\footnotetext{
12 The MSV model in this paper was used also by Shi and Jiang (2016) to evaluate the efficiency of an index insurance in hedging revenue risk against extreme weather conditions in paddy production in China. See their Appendix B for details. Note that the MSV calculation uses the count of all observations, to account for the share of downside risk observations in the full sample of realised crop production.

${ }^{13}$ Pseudo-significance for the GWR refers to the t-statistic for the coefficient associated with a (local) regression point.
} 
evidence of an association between changes in paddy yields and the changing levels of the weather indices (PDSI, ENSO, and DMI). In addition, the spatial regression models also show the highly significant coefficients $\rho$ and $\lambda$ that define the spatial dependence in the observed variables as shown in Table 2.

Results for the benchmark GWR Panel model that accounts for the spatial differences in the estimated coefficients are presented in Table 2 column 4. Since we cannot show the estimated coefficient for each district, we average the coefficients by quartiles. These confirm the spatial heterogeneity we hypothesized, as the interquartile ranges of the coefficients (for PDSI, ENSO, and DMI) are all larger than two times the standard errors of the global fixed effect model and the spatial regression models.

Table 2 Estimation Results Fixed Effect, Spatial Regression Model and GWR Panel of 428 Districts in Indonesia during 1990 - 2013

\begin{tabular}{|c|c|c|c|c|c|c|}
\hline \multirow{2}{*}{$\begin{array}{l}\text { Dependent Variable: } \\
\text { Paddy Yield (Ton/Ha) }\end{array}$} & \multirow{2}{*}{$\begin{array}{c}\text { Fixed Effect } \\
\text { (1) }\end{array}$} & \multirow{2}{*}{$\begin{array}{c}\text { Spatial Lag } \\
\text { (SAR) } \\
(2)\end{array}$} & \multirow{2}{*}{$\begin{array}{c}\text { Spatial Error } \\
\text { (SEM) } \\
(3)\end{array}$} & \multicolumn{3}{|c|}{ GWR Panel } \\
\hline & & & & Q1 & $\begin{array}{c}\text { Median } \\
(4) \\
\end{array}$ & Q3 \\
\hline PDSI & $\begin{array}{c}0.0307^{* * *} \\
(0.00172)\end{array}$ & $\begin{array}{c}0.0184^{* * *} \\
(0.00146)\end{array}$ & $\begin{array}{c}0.0264^{* * *} \\
(0.00220)\end{array}$ & 0.0020 & .0239 & 0.0457 \\
\hline ENSO $\left({ }^{\circ} \mathrm{C}\right)$ & $\begin{array}{l}-0.0271^{* * *} \\
(0.00576)\end{array}$ & $\begin{array}{l}-0.00955^{*} \\
(0.00486)\end{array}$ & $\begin{array}{l}-0.0251^{* * *} \\
(0.00755)\end{array}$ & -0.0855 & -0.0378 & 0.0112 \\
\hline ENSO2 & $\begin{array}{l}-0.0741^{* * *} \\
(0.00480)\end{array}$ & $\begin{array}{l}-0.0383^{* * *} \\
(0.00409)\end{array}$ & $\begin{array}{l}-0.0530^{* * *} \\
(0.00652)\end{array}$ & & & \\
\hline DMI $\left({ }^{\circ} \mathrm{C}\right)$ & $\begin{array}{c}0.311^{* * *} \\
(0.00911)\end{array}$ & $\begin{array}{c}0.165^{* * *} \\
(0.00810)\end{array}$ & $\begin{array}{c}0.226^{* * *} \\
(0.0126)\end{array}$ & 0.2354 & 0.2993 & 0.3416 \\
\hline Constant & $\begin{array}{c}4.080^{* * *} \\
(0.00458)\end{array}$ & & & & & \\
\hline$\rho$ & & $\begin{array}{c}0.476^{* * *} \\
(0.00856)\end{array}$ & & & & \\
\hline$\lambda$ & & & $\begin{array}{c}0.477^{* * *} \\
(0.00887)\end{array}$ & & & \\
\hline$\sigma^{2}$ & & $\begin{array}{c}0.0809^{* * *} \\
(0.00115)\end{array}$ & $\begin{array}{c}0.0819^{* * *} \\
(0.00117)\end{array}$ & & & \\
\hline$N$ & 10272 & 10272 & 10272 & & 10272 & \\
\hline R-squared & 0.141 & 0.151 & 0.139 & & 0.764 & \\
\hline Number of District & 428 & 428 & 428 & & 428 & \\
\hline Number of Years & 24 & 24 & 24 & & 24 & \\
\hline
\end{tabular}

The spatial distributions of the GWPR coefficient estimates of the PDSI are presented in Figure 1, showing a strong relation between variability of drought indices PDSI and paddy yields in several regions in Eastern and South Eastern part of Sumatra, most of Sulawesi, the middle and southern part of Kalimantan and the eastern part of Papua. A comparison of Figures 2 and 3 shows that for the GWPR model estimates, there are more pseudosignificant coefficients for PDSI (214 districts - about 50\% of the total observed districts) 
than in the local regression model where each district is estimated separately. The results of the local regressions of each district show that the PDSI coefficient is statistically significant in 140 districts (compared to 214 for the GWPR).

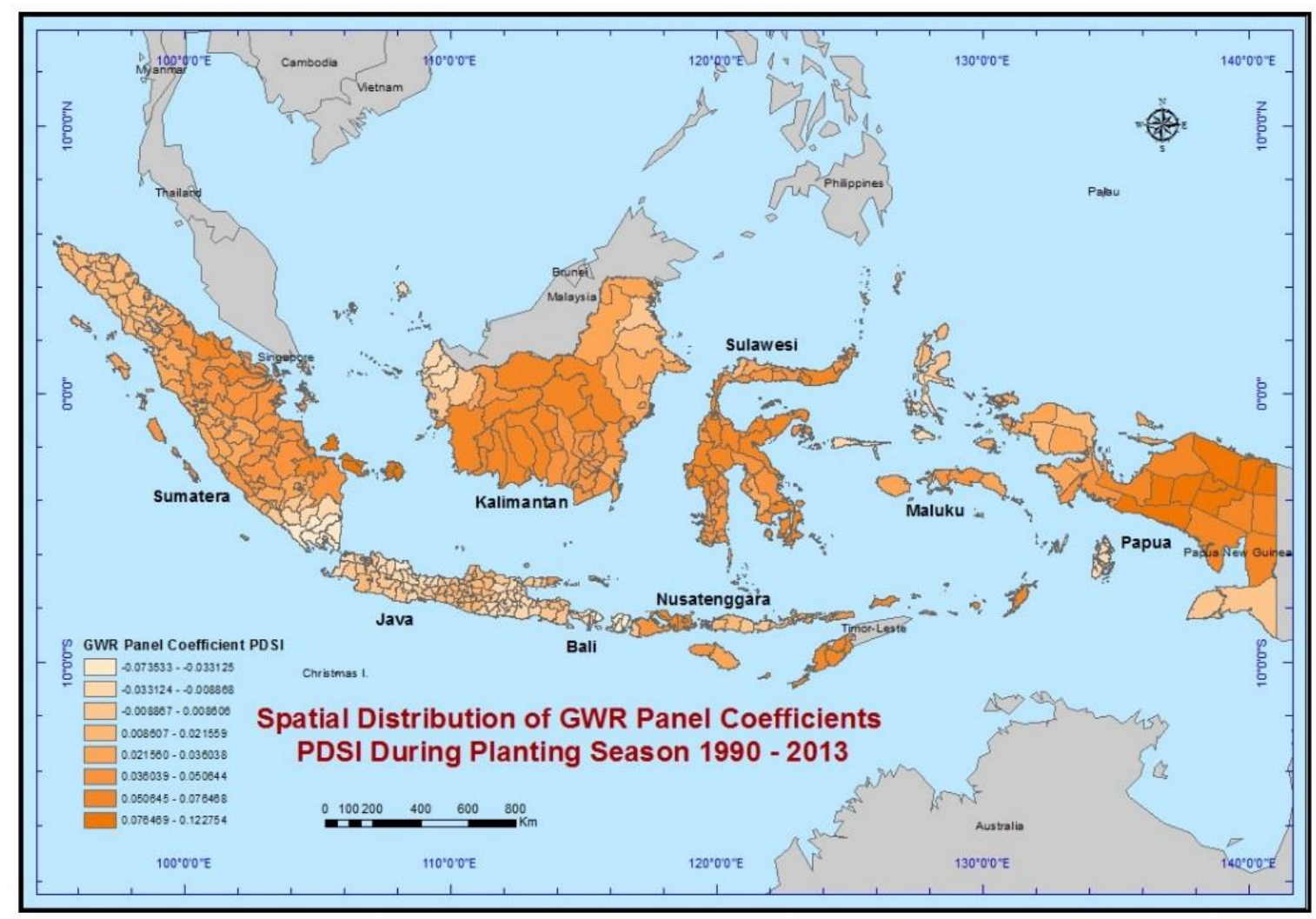

Figure 1 Spatial distribution of GWR panel coefficient estimate PDSI planting season

In Figure 4 that zooms in on the significant regions, we see that paddy yields are positively correlated with the PDSI in almost all districts in Sulawesi, the districts in the central part of Sumatra, central to southern part of Kalimantan, and the eastern part of Papua. 


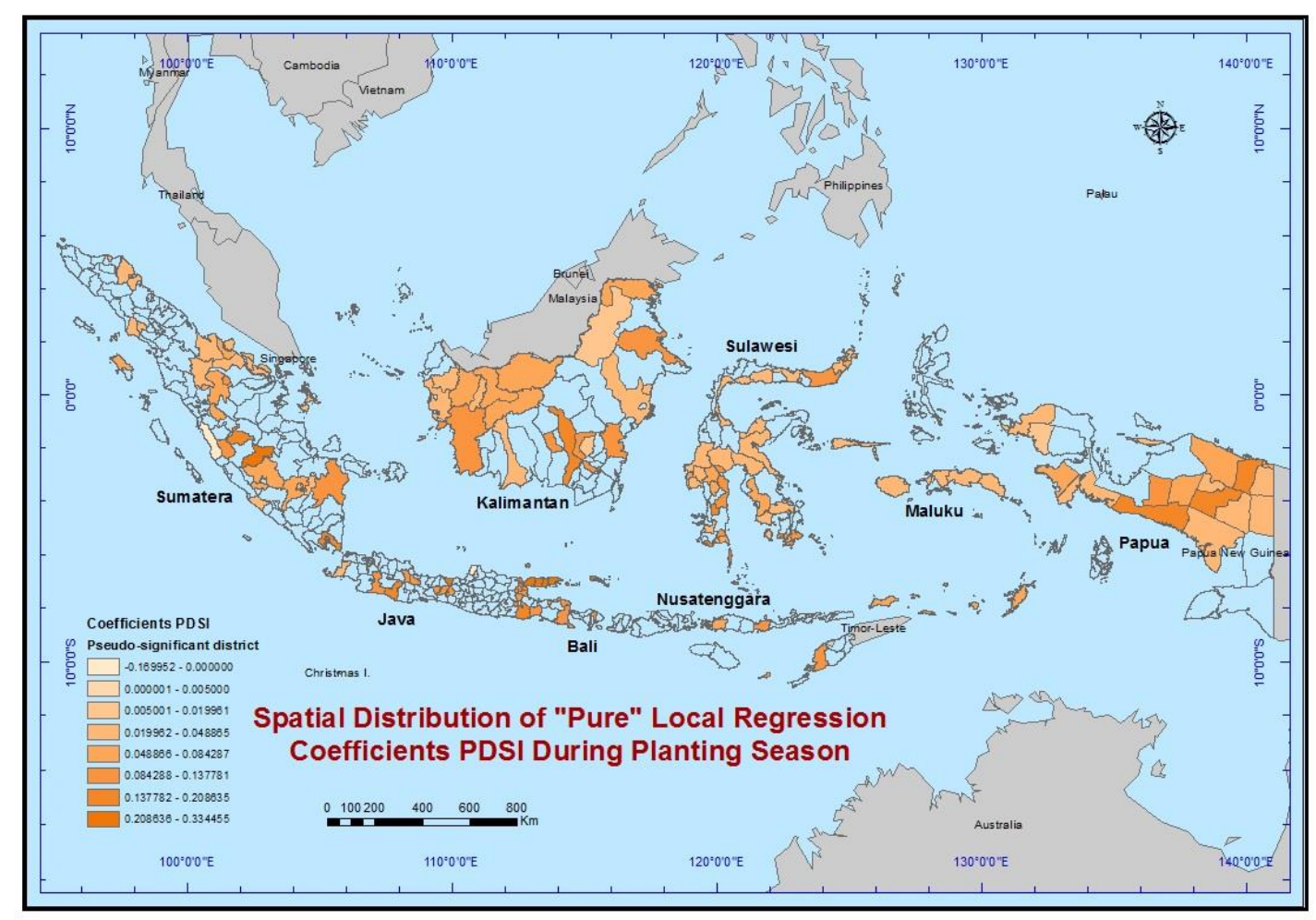

Figure 2 Spatial distribution of local coefficient estimate PDSI planting season of districts that are pseud-significant at $5 \%$ significance level

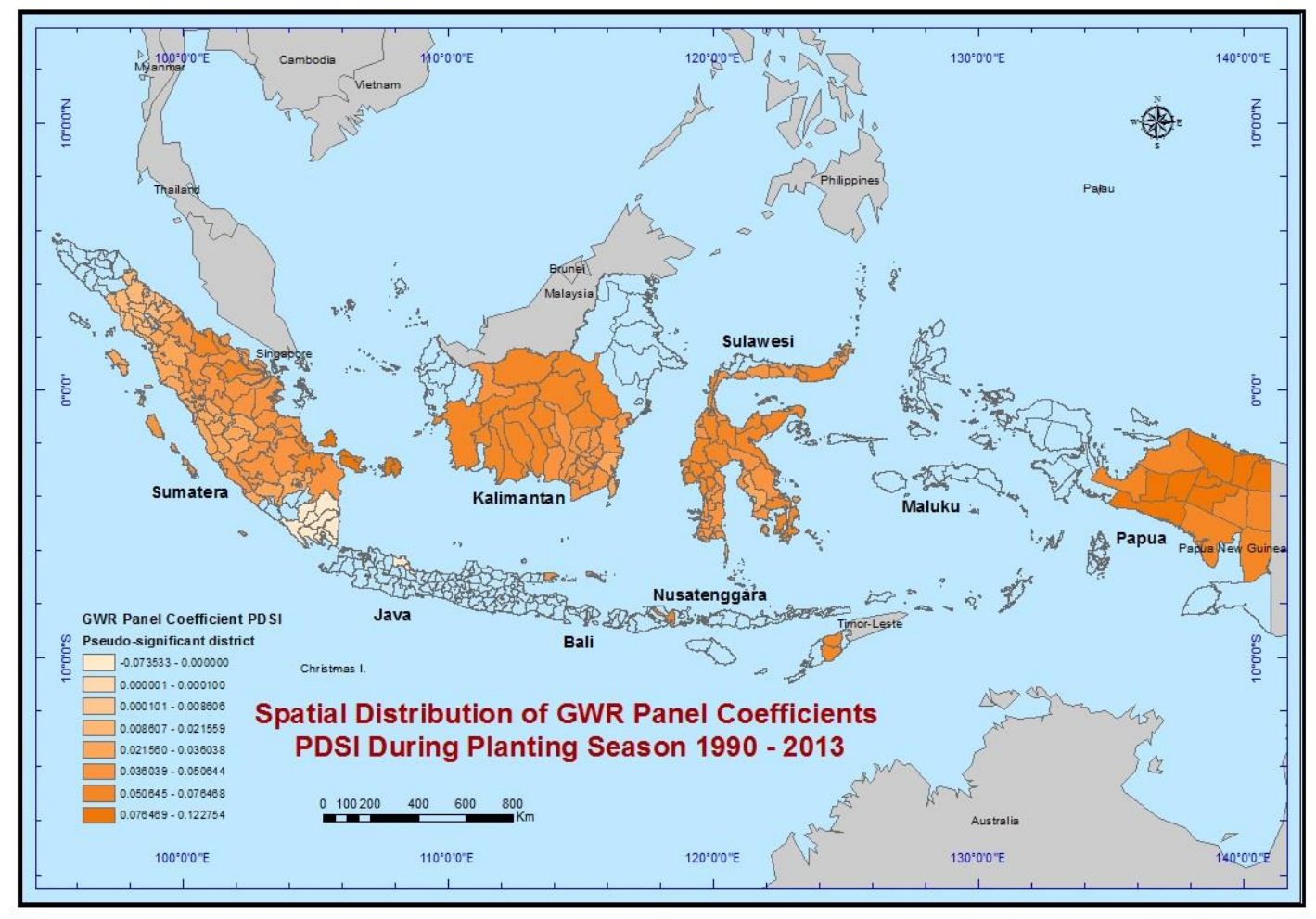

Figure 3 Spatial distribution of GWR panel coefficient estimate PDSI planting season of districts that are pseudo-significant at 5\% significance level 

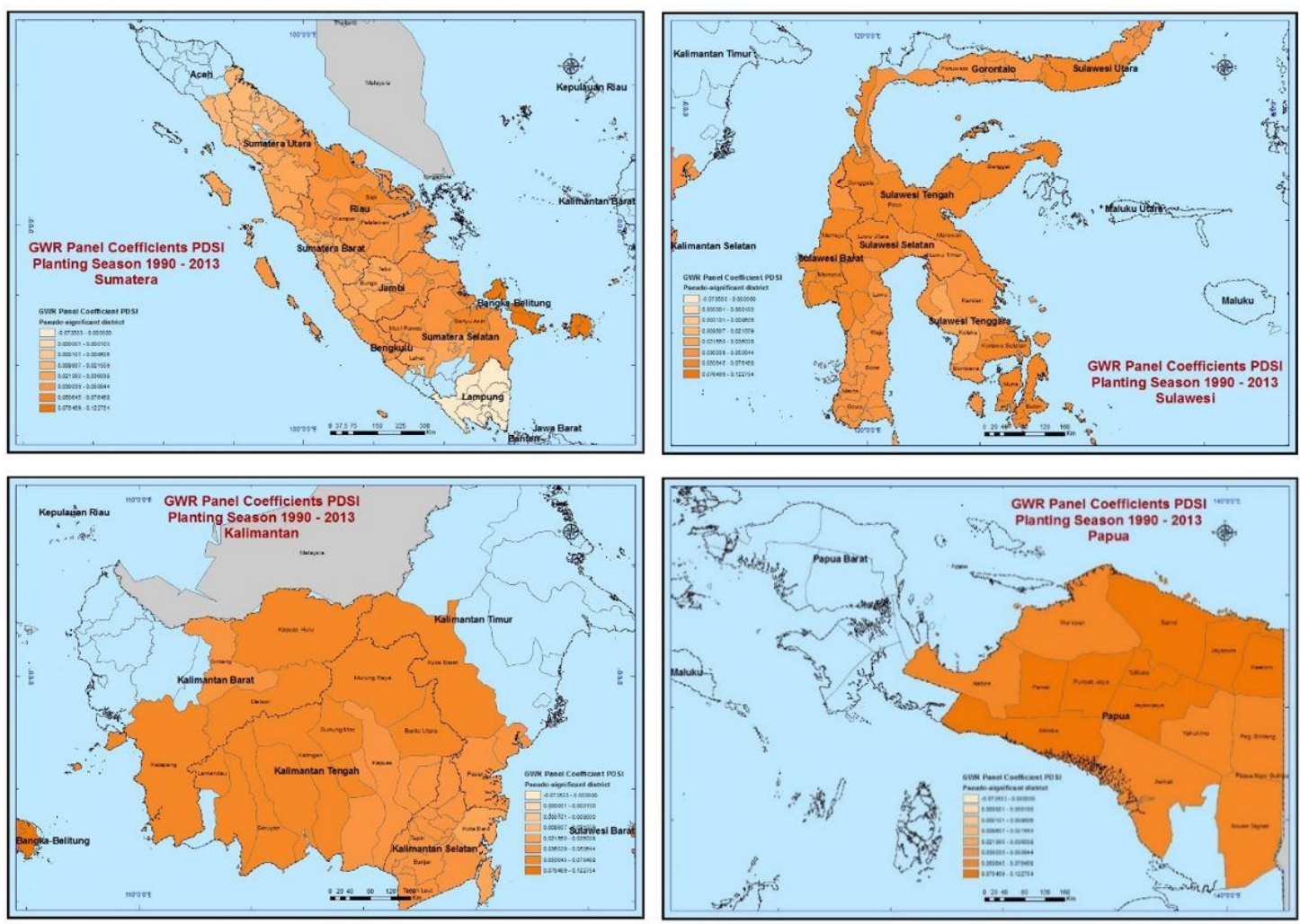

Figure 4 Spatial distribution of GWR panel coefficients PDSI in planting season of districts in major islands that are pseudo-significant at 5\% significance level

The PDSI index is not statistically associated with paddy yields in districts in Java, Bali, Nusa Tenggara, and Maluku, except within a few districts that appear randomly distributed across these islands (Figure 3). For Java and Bali, this is most likely due to the extensive irrigation networks that are in use there, ameliorating the effects of drought conditions on paddy productivity. For the districts in Maluku and Nusa Tenggara, the lack of statistical power may be hindering any precise identification, as these districts are in small islands and therefore have fewer neighboring districts and consequently fewer degrees of freedom.

It is interesting to note-see Annex 9-that paddy yields are correlated positively with the Indian El Niño (DMI) in most of Kalimantan and all parts of Sumatra, Java-Bali and Sulawesi while there is no evidence that climate impact exists at districts further away in northern Kalimantan, all Maluku and all of Papua. For the Pacific El Niño (ENSO), the phenomenon's impact on paddy yield is found in the southern part of Sumatra and in only a small part of northern Java while a moderate effect is experienced in the northern part 
of Sumatra and much of Java (Annex 10). ${ }^{14}$ A complete set of of estimated parameters for each district from the GWPR is available in Annex 14.

\subsection{Designing a weather index insurance product}

Building on our findings that establish a substantial degree of correlation between the observed weather indices and crop yield (R-squared 0.764 ), we now turn to the design of the WII product we propose using the PDSI index. We can now, using our previous results, identify the districts for which such an insurance product is viable. In this case, payments will be triggered when the PDSI exceeds a predetermined threshold level (the Trigger) until a maximum indemnity payment level is reached at a higher PDSI (the Exit). At the PDSI range between the Trigger and Exit levels, we assumed that paddy yield decreases progressively resulting in more and more crop loss (and more and more indemnity payment).

To determine the underlying threshold points (Trigger and Exit), we first cluster the districts with pseudo-significant coefficient estimates for the PDSI in the major islands where GWR Panel results show statistical significance. These include districts in Sulawesi, Kalimantan, Sumatra, and Papua. We don't include actuary calculation for districts in Java, Bali, Nusa Tenggara, and Maluku since the GWPR estimates show little evidence that PDSI is closely related to yield losses in these regions.

Next, we apply the model-based cluster analysis described in the previous section in order to group lower PDSI values during planting season (September to December) and lower crop yields to determine the Trigger and Exit level of the index insurance; as in (Choudhury et al., 2016).

Figure 5 shows the results of model-based cluster analysis that produces two clusters in the sample islands Sulawesi and Sumatra (result for other islands are presented in Annex 14). The lower (red) cluster represents the combination of lower PDSI and paddy yield, hence we set this level as the "trigger". We set the expected value of PDSI in the lower (red) cluster as the predetermined threshold for the payment trigger where indemnity payment start to be provided by the insurance policy and the minimum value of PDSI in

\footnotetext{
14 These El Niño results are consistent with previous findings (Naylor et al., 2001; D'Arrigo \& Wilson, 2008).
} 
the lower (red) cluster as the predetermined threshold for the exit where total suminsured is reached.
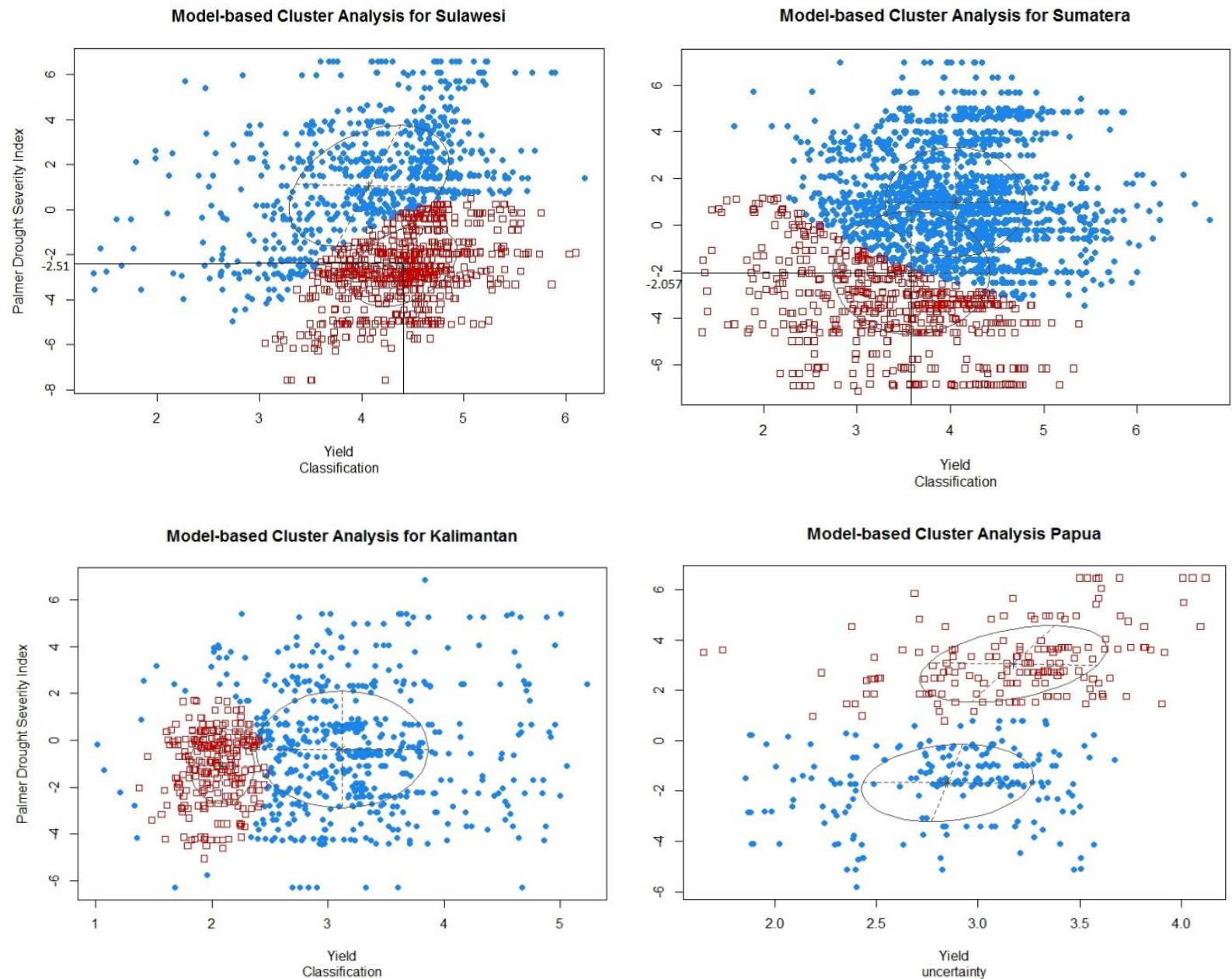

Figure 5 Trigger of underlying index PDSI for districts in the island of Sulawesi, Sumatra, Kalimantan and Papua using model-based cluster

Once we set the underlying index, we calculate the premium for the index insurance that will be applied at each major island. We propose a uniform pricing strategy for each major island, rather than specific pricing for every district, as its implementation is significantly more convenient; and we believe its simplicity will be attractive for commercial insurers. After all, an important feature of index insurance distinguishing it from conventional insurance is its simplicity. Unique pricing for every district may make such a program more difficult to implement because of public and political pressures.

Using equation (7), we calculate the average future payouts (EAL) during the 24 years of observation, using the historically observed drought index (1990-2013) - assuming the 
insurance scheme was offered in that period. We then determine the premium by calculating the present value of these payouts as described in equation (6). ${ }^{15}$

We set the following assumptions in analysing the risk reduction achieved by the proposed WII in smoothing farmers' revenue during drought: We assume the price of paddy (GKG/dry unhusked rice) is constant - Rp. 4,000/kg, and set the maximum insured amount at Rp. 2,000,000/Ha - which is equal to the averaged costs for inputs (seed, fertilizer, pesticide) and land lease of rain-fed paddy farming. ${ }^{16}$ The parameters of the proposed insurance contracts are presented in Table 3.

Table 3 Parameters of Weather Index Insurance Using Drought Indices PDSI for Rice Production in Major Islands in Indonesia

\begin{tabular}{lcccccc}
\hline Island & $\begin{array}{c}\text { Insured } \\
(\mathrm{Rp})\end{array}$ & $\begin{array}{c}\text { Trigger } \\
(\mathrm{PDSI})\end{array}$ & $\begin{array}{c}\text { Exit } \\
\text { PDSI) }\end{array}$ & $\begin{array}{c}\text { Tick (IDR / } \\
\text { point index) }\end{array}$ & $\begin{array}{c}\text { Average Payout } \\
(\mathrm{Rp})\end{array}$ & $\begin{array}{c}\text { Premium } \\
(\mathrm{Rp})\end{array}$ \\
\hline Sumatra & $2,000,000$ & -2.057 & -6.907 & 412,371 & 22,104 & 21,000 \\
Kalimantan & $2,000,000$ & -1.092 & -6.265 & 386,623 & 112,661 & 107,000 \\
Sulawesi & $2,000,000$ & -2.510 & -7.583 & 394,244 & 24,504 & 24,000 \\
Papua & $2,000,000$ & -1.675 & -5.808 & 483,910 & 37,525 & 36,000 \\
\hline
\end{tabular}

\subsection{The efficiency of weather index insurance using the PDSI}

Lastly, we examine whether the drought index insurance as designed above has the potential to assist farmers in dealing with the income variability they face because of weather extremes. First, we compare the actual revenue from paddy farming without index insurance to the hypothetical revenue from paddy farming with index insurance, all calculated for the same observation period: 1990-2012; see Figure 6.

We observe that the variance of crop revenue from paddy farming with and without insurance are distinct and that farmers with insurance appear to be able to stabilize their income during drought events like in 1991 - 1995 in Mamasa district (top panel). The same situation also occurs over 1991 - 1993 for Aceh Singkil district (middle panel), and in many years between 1990 and 2008 for Ketapang district (bottom panel).

\footnotetext{
${ }^{15}$ See Choudhury et al. (2016).

16 (http://www.bps.go.id/linkTabelStatis/view/id/1855)
} 

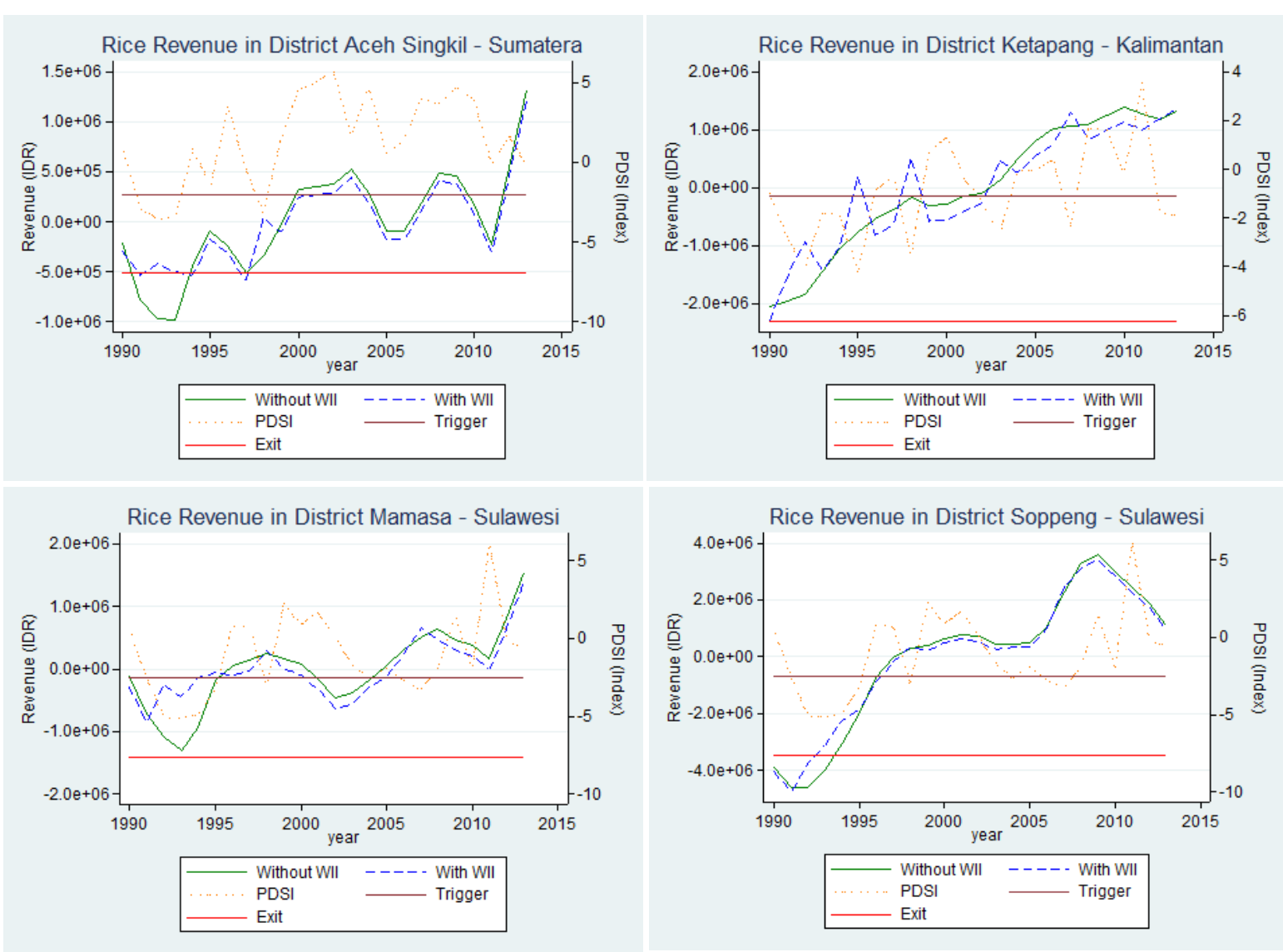

Figure 6 Paddy revenues without and with index insurance in selected districts in Sulawesi, Kalimantan, and Sumatera

Next, we present in Table 4 a selection of results for 20 districts obtained from our revenue calculations and the change in revenue variability (the standard deviation of revenue), with and without insurance, based on the historical observations. The districts selected are the ones where the variability declined between $7 \%-23.7 \%$. Total revenue per hectare without insurance, $R_{0}$, is derived by multiplying unit paddy price with predicted yield. Revenue paddy farming per hectare, $R_{1}$, is calculated from the sum of $R_{0}$ plus indemnity payment from the index insurance and minus premium. Annex 15 presents the results for all other districts.

It should be noted that the aim of insurance is not to increase the expected revenue, but to reduce its volatility (typically at the cost of somewhat reduced expected revenue). In this case, we observe some districts for which revenue is indeed decreasing and some in which revenue is increasing. There are two reasons for this: (1) The triggers and exit points are calculated per island, rather than per district, so some districts do also experience an increase in expected revenue. (2) We assumed districts are paying 
actuarially fair premiums (i.e., the government is subsidising the overhead costs of running the insurance scheme).

Table 4 Comparison revenue with and without insurance in selected districts

\begin{tabular}{|c|c|c|c|c|c|}
\hline No & District & Island & $\begin{array}{l}\text { Without Insurance } \\
\text { (IDRx1000) }\end{array}$ & $\begin{array}{l}\text { With Insurance } \\
(\text { IDRx1000) }\end{array}$ & SD Change \\
\hline 1 & Mamasa & Sulawesi & $16,445(625.9)$ & $16,588(477.6)$ & $-23.70 \% * *$ \\
\hline 2 & Polewali Mandar & Sulawesi & $19,403(726.1)$ & $19,546(599.4)$ & $-17.45 \% * *$ \\
\hline 3 & Parigi Moutong & Sulawesi & $19,478(794.3)$ & $19,657(679.5)$ & $-14.46 \% * *$ \\
\hline 4 & Gorontalo & Sulawesi & $18,222(782.6)$ & $18,344(703.7)$ & $-10.07 \% * *$ \\
\hline 5 & Pohuwato & Sulawesi & $18,964(1,044.4)$ & $19,144(942.5)$ & $-9.76 \% * *$ \\
\hline 6 & Pare-pare & Sulawesi & $17,326(1,349.3)$ & $17,469(1,243.6)$ & $-7.83 \% * *$ \\
\hline 7 & Soppeng & Sulawesi & $21,129(2,458.5)$ & $21,272(2,272.9)$ & $-7.55 \% * *$ \\
\hline 8 & Barru & Sulawesi & $19,031(1,602.7)$ & $19,174(1,488.3)$ & $-7.14 \% * *$ \\
\hline 9 & Aceh Singkil & Sumatra & $13,885(532.7)$ & $13,946(429.2)$ & $-19.43 \% *$ \\
\hline 10 & Langsa & Sumatra & $15,663(565.0)$ & $15,725(473.5)$ & $-16.20 \% *$ \\
\hline 11 & Medan & Sumatra & 17,737 (559.9) & $17,799(469.4)$ & $-16.17 \% *$ \\
\hline 12 & Kampar & Sumatra & $11,367(1,307.9)$ & $11,718(1,104.8)$ & $-15.53 \% \%^{* * *}$ \\
\hline 13 & Aceh Tamiang & Sumatra & $16,783(669.7)$ & $16,845(575.2)$ & $-14.12 \% *$ \\
\hline 14 & Pakpak Bharat & Sumatra & $13,357(659.0)$ & $13,419(576.1)$ & $-12.57 \% *$ \\
\hline 15 & Sijunjung & Sumatra & $15,983(1,266.8)$ & $16,176(1,114.0)$ & $-12.06 \%{ }^{* *}$ \\
\hline 16 & Tebing Tinggi & Sumatra & $17,854(600.0)$ & $17,916(534.7)$ & $-10.88 \% *$ \\
\hline 17 & Pekanbaru & Sumatra & $10,816(2,510.6)$ & $11,168(2,311.9)$ & $-7.91 \% * * *$ \\
\hline 18 & Aceh Tenggara & Sumatra & $16,550(1,009.5)$ & $16,612(932.6)$ & $-7.62 \% *$ \\
\hline 19 & Samosir & Sumatra & $18,346(827.8)$ & $18,408(769.2)$ & $-7.07 \% *$ \\
\hline 20 & Ketapang & Kalimantan & $10,847(1,109.2)$ & $11,005(1,020.5)$ & $-8.00 \% * *$ \\
\hline
\end{tabular}

Note: Revenues are average values during 1990-2013 and standard deviations (SD) are shown in parentheses. The table shows results from selected districts with regards significant SD decrease. See Annex 15 for results for all districts. Unit Revenue and Standard Deviation is in Indonesian Rupiah (IDR). *, $* *, * * *$ denote $10 \%, 5 \%$ and $1 \%$ statistical significance for the difference between the standard deviations of revenue with and without insurance.

Summarizing Annex 15, we found that reduction of farm income volatility occurs in major parts of Sulawesi (79\% of districts), Papua (64\% of districts), Sumatra (51\% of districts) and some part of Kalimantan (29\% of districts). In Java's districts, the variability actually increased by up to $40 \%$, indicating that the insurance contract is not effective at reducing risk. This incongruence may be because the PDSI is not a good predictor for agricultural productivity in an irrigated and intensely managed agricultural sector such as paddy rice in Java; in these cases, index insurance using a signal like the PDSI does not work well. 
Finally, we apply the MSV model to assess how WII can reduce income exposures during drought. We analyse revenue at-risk, V, with and without WII at a different level of $k$ (the measure of risk-averseness) at the same selected districts as in Table 4. As can be seen in Table 5, farmers with insurance (WII) have higher revenue at-risk (V). V is described in eq. 9, but essentially it is the expected revenue minus a weighted (by k) semi-variance (the variance of downside risk years). Thus, a higher k implies higher risk-averseness.

Table 5 Efficiency test using mean-semivariance model

\begin{tabular}{|c|c|c|c|c|c|c|}
\hline & \multicolumn{2}{|c|}{$k=0.1$} & \multicolumn{2}{|c|}{$k=0.2$} & \multicolumn{2}{|c|}{$k=0.3$} \\
\hline & V-no WII & V - WII & V- no WII & V-WII & V- no WII & V-WII \\
\hline Bitung & 16.423 & 16.573 & 16.400 & 16.559 & 16.378 & 16.544 \\
\hline Minahasa Utara & 13.866 & 13.933 & 13.848 & 13.920 & 13.829 & 13.906 \\
\hline Sintang & 19.377 & 19.525 & 19.352 & 19.505 & 19.327 & 19.485 \\
\hline Dumai & 15.643 & 15.709 & 15.624 & 15.694 & 15.604 & 15.679 \\
\hline Rokan Hilir & 17.718 & 17.783 & 17.698 & 17.768 & 17.679 & 17.753 \\
\hline Bengkalis & 11.327 & 11.682 & 11.287 & 11.647 & 11.247 & 11.611 \\
\hline Siak & 19.450 & 19.633 & 19.423 & 19.610 & 19.396 & 19.586 \\
\hline Boalemo & 16.758 & 16.824 & 16.734 & 16.804 & 16.709 & 16.783 \\
\hline Pekanbaru & 13.336 & 13.402 & 13.315 & 13.384 & 13.294 & 13.367 \\
\hline Majene & 15.938 & 16.135 & 15.892 & 16.094 & 15.846 & 16.053 \\
\hline Polewali Mandar & 17.836 & 17.900 & 17.818 & 17.884 & 17.799 & 17.869 \\
\hline Mamasa & 18.199 & 18.324 & 18.175 & 18.303 & 18.152 & 18.283 \\
\hline Rokan Hulu & 18.933 & 19.117 & 18.903 & 19.090 & 18.872 & 19.063 \\
\hline Kampar & 10.807 & 10.968 & 10.766 & 10.930 & 10.726 & 10.892 \\
\hline Tana Toraja & 10.733 & 11.091 & 10.650 & 11.013 & 10.567 & 10.936 \\
\hline Barito Timur & 17.275 & 17.421 & 17.224 & 17.373 & 17.174 & 17.325 \\
\hline Pinrang & 16.508 & 16.573 & 16.466 & 16.535 & 16.425 & 16.496 \\
\hline Banggai & 21.034 & 21.185 & 20.939 & 21.099 & 20.843 & 21.012 \\
\hline Tojo Una-una & 18.967 & 19.114 & 18.903 & 19.054 & 18.839 & 18.993 \\
\hline Luwu Utara & 18.323 & 18.387 & 18.300 & 18.367 & 18.276 & 18.346 \\
\hline
\end{tabular}

Note: Revenue at-risk $\mathrm{V}$ without and with insurance at a different level of $k$ (in million IDR- Indonesian Rupiah). Revenue at-risk $\mathrm{V}$ with insurance is higher than revenue at-risk $\mathrm{V}$ without insurance. A higher revenue at-risk $V$ value corresponds to lower risk exposure (Shi \& Jiang, 2016).

As described in equation (9), revenue at-risk (V) has a negative link with the (semi) variance. Therefore, a higher value of revenue at-risk (V) corresponds to the lower (semi) variance of revenue. We can also see in Table 5 that the higher the value of $\mathrm{k}$ (the weight 
placed on the semi-variance in calculating V), the larger the gap between WII and nonWII farmers. This may not be surprising, and it indicates that more risk-averse farmers may be more interested in WII than less risk-averse farmers.

\subsection{Conclusion, caveats, and recommendations}

The impacts of natural hazards on livelihoods have increased substantially in the past few decades worldwide. A number of factors are at play, but at least some of this increase is attributable to increasing weather risk caused by climatic changes; these have particular effects in middle- and low-income areas that are more reliant on agricultural production. Given the increased risk to the agricultural sector in a country such as Indonesia, disaster risk-transfer strategies can, therefore, be an important tool to reduce the impact of natural hazards on farmers' incomes and its variability.

Weather index insurance (WII) may be one form of insurance that can be productively used to accomplish some of the goals set out by the 2015 Sendai Framework on Disaster Risk Reduction. As the Sendai Framework recognised, insurance can be a tool that enables the transfer of risk from vulnerable households to established financial institutions and markets. Weather index insurance has been piloted and implemented in several developing countries, and according to its advocates, it can provide an effective approach to improve emergency response to weather-related catastrophes. Recent experiences in, for example, Mongolia and Peru for cold waves and floods, respectively, demonstrate this potential (UNFCCC, 2008; Collier et al., 2009).

The penetration rate for these schemes in emerging markets and developing countries are still, however, very low. Even in successful programs, the penetration is quite low; the take up rate for the index-based Mongolian Livestock scheme mentioned above is only about $10 \%$ of herding households, in spite of significant subsidies. Public-private partnership insurance schemes can, to some extent, address the poor financial performance of public insurance and overcome the market failures plaguing private insurers by enlarging market uptake (sometime even mandating it). Index-based insurance such as the one proposed here is a response to information asymmetries and high verification costs faced by conventional indemnity-based agricultural insurance. 
Still, these new products are challenged by lack of trust, and enabling regulations are considerably underdeveloped, including in Indonesia.

Here, we investigated the relationships between paddy yield and weather indices during the planting season by exploiting the spatio-temporal variation of both, including applying a Geographically Weighted Panel Regression method to account better for the spatial component of this variation than previous studies have achieved. This allowed us to identify the Indonesian regions in which a WII scheme would be viable and most effective. We found that paddy yield variations in many districts in Sulawesi are strongly positively associated with the PDSI index. The same was also true in Central Sumatra, Central and Southern Kalimantan, and Eastern Papua. We did not establish a similar association between the PDSI and rice productivity over much of the rest of Indonesia, including most districts in Java, Bali, Nusa Tenggara, and Maluku. As such, a viable WII scheme for rice in Indonesia should be restricted to these specific locations where the index itself is informative and basis risk is minimal.

The detailed spatial information, on varying responses of paddy to fluctuations in weather indices, allows us to tailor specific WII schemes for different targeted districts in Indonesia. This island-specific tailoring-most importantly by setting island-specific trigger and exit points-results in more effective risk reduction with less basis risk.

What type of scheme may be appropriate for islands in which the PDSI is not tightly correlated with rice production is an open question. Alternative weather measures or satellite-based observational data can be explored as potential parametric anchors. We leave these possibilities for future research.

Beyond the use of our finding in constructing WII programs, one can also use our evidence on the correlations between the PDSI and rice farm income, to develop other risk reduction programs. For example, the prioritization of investment in irrigation infrastructure may be guided by the relationship we uncovered between the drought index (the PDSI) and crop productivity in some districts and not others. Other conditional cash transfer programs can also be tied specifically to these weather and climate indices. $^{17}$

\footnotetext{
${ }^{17}$ For an overview of these cash transfer programs, see (Kwon \& Kim, 2015).
} 
We conclude that a PDSI index insurance program may be suitable for implementation in more drought-sensitive areas like in Sulawesi, some parts of Sumatra and some smaller parts of Kalimantan. We found that the insurance contract reduced the decline in revenues of participating farmers during drought periods in rice production districts in Sulawesi such as in Bitung, Minahasa Utara, Polewali Mandar and in Sumatran districts such as in Dumai, Rokan Hilir, and Bengkalis. We emphasize Sulawesi as a priority for pilot implantation of this program because of the strong evidence of the association between the drought index variability and paddy yield in almost all districts on that very big island. Our findings also show that WII in Sulawesi has the highest financial potential of hedging risks and decreasing the volatility of income.

It is also important to remember that insurance is not the only tool for risk transfer in the face of weather shocks, in Indonesia and elsewhere. Skoufias (2003) provides an overview of various alternative informal risk sharing mechanisms that are popular in low- and middle-income countries; while Berloffa and Modena (2013) investigate the evidence for these mechanisms using the Indonesian Family Life Survey data. An investigation of these alternative risk sharing mechanisms is beyond the scope of this paper, but they are clearly also important (Skoufias et al., 2012). However, it is possible that implementation of a WII scheme, such as the one we propose, will modify the alternative incentives and mechanisms that are detailed in these papers. A comprehensive welfare analysis of the implantation of such a WII scheme is therefore quite challenging and is clearly a goal which we can now only aspire to reach.

We end by noting that there are only a very limited number of papers using geographically-weighted regression techniques in any economic context. While no one has applied this spatial modelling approach to design WII schemes, as far as we are aware, the same suite of methods can also be used in other fields of investigation and research where the spatial distribution of economic activity is important. 


\section{Annexes Chapter TWO}

Annex 1 Yield and detrended yield of selected districts
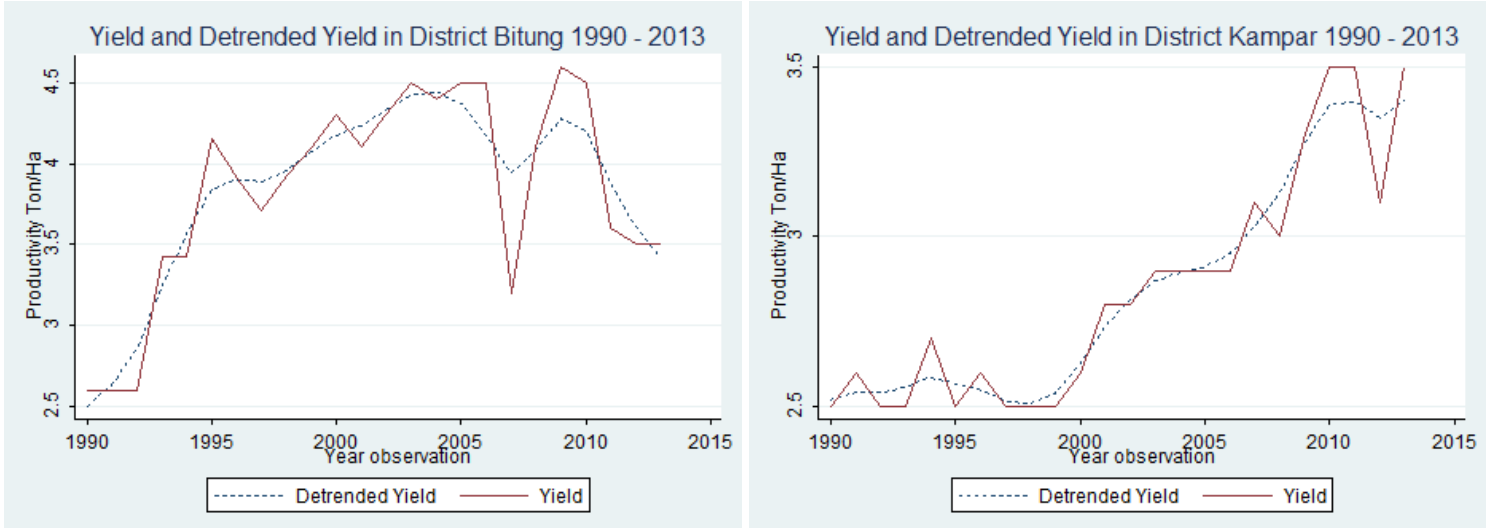

Yield and Detrended Yield in District Sintang, West Kalimanta

Yield and Detrended Yield in District Sintang, West Kalimanta
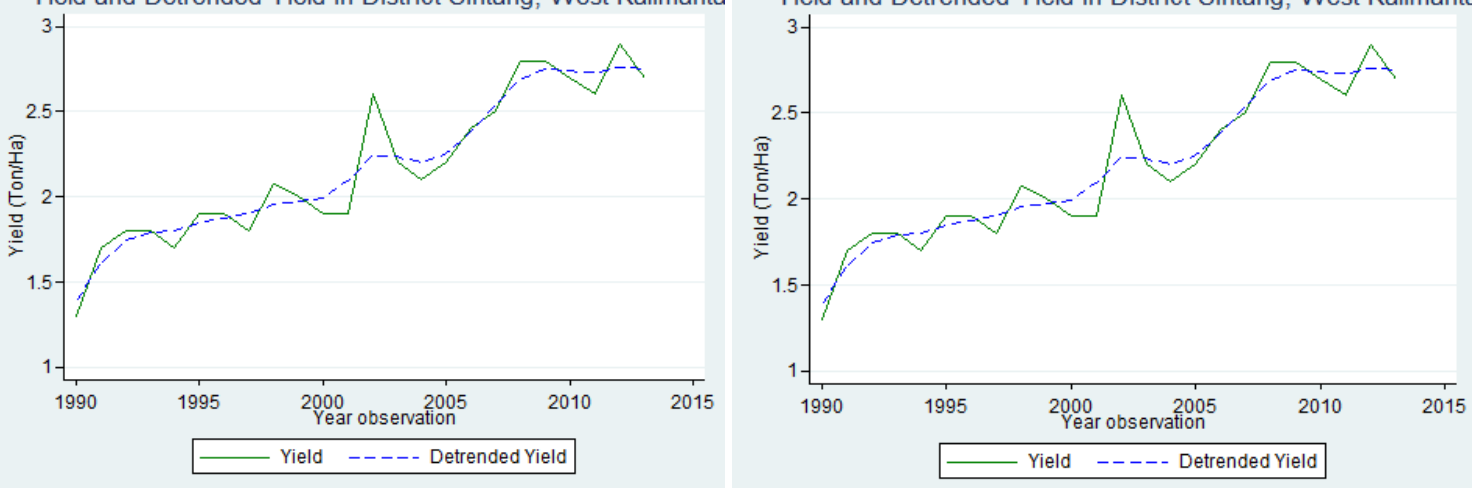

Annex 2 Paddy Yield (Kg/Ha) across 428 districts in Indonesia during 1990 - 2013

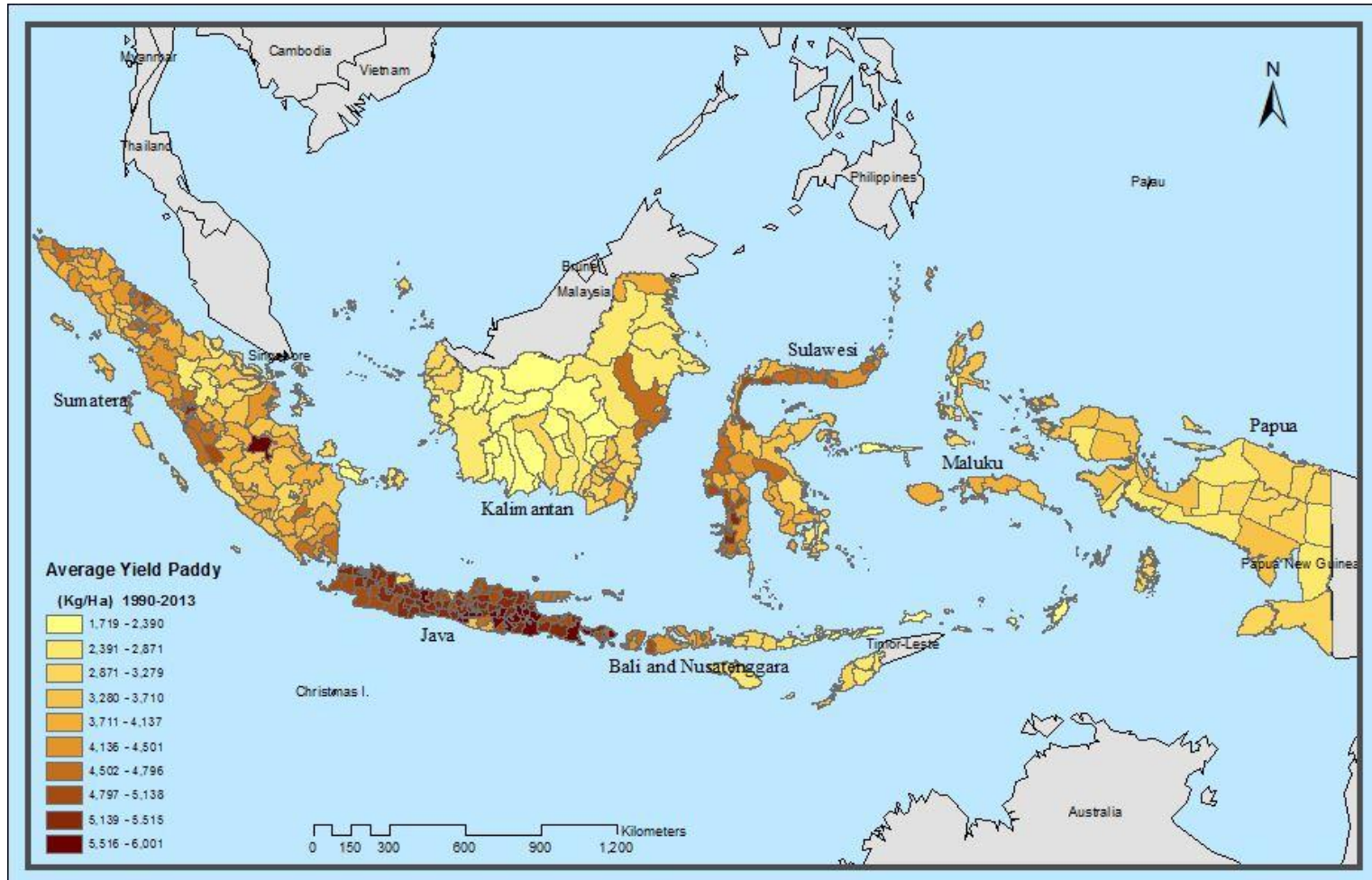

Note: the value is average productivity of paddy for the period of 24 years (1990 - 2013) 


\section{Annex 3 Self-calibrated Palmer Drought Severity Index (PDSI) gridded area}

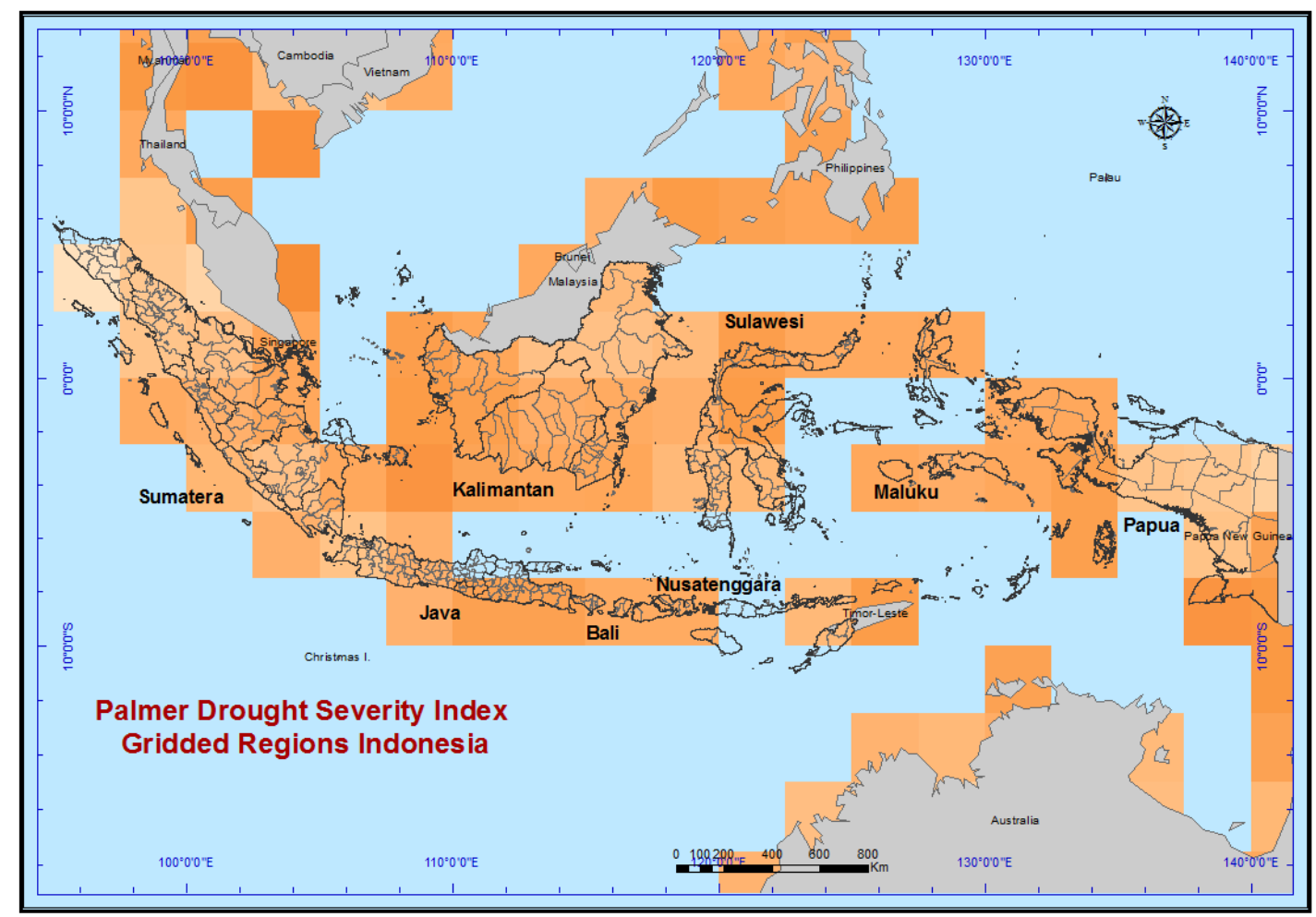

Annex 4 Average PDSI value in planting season (Sept-Dec) period 1990-2013

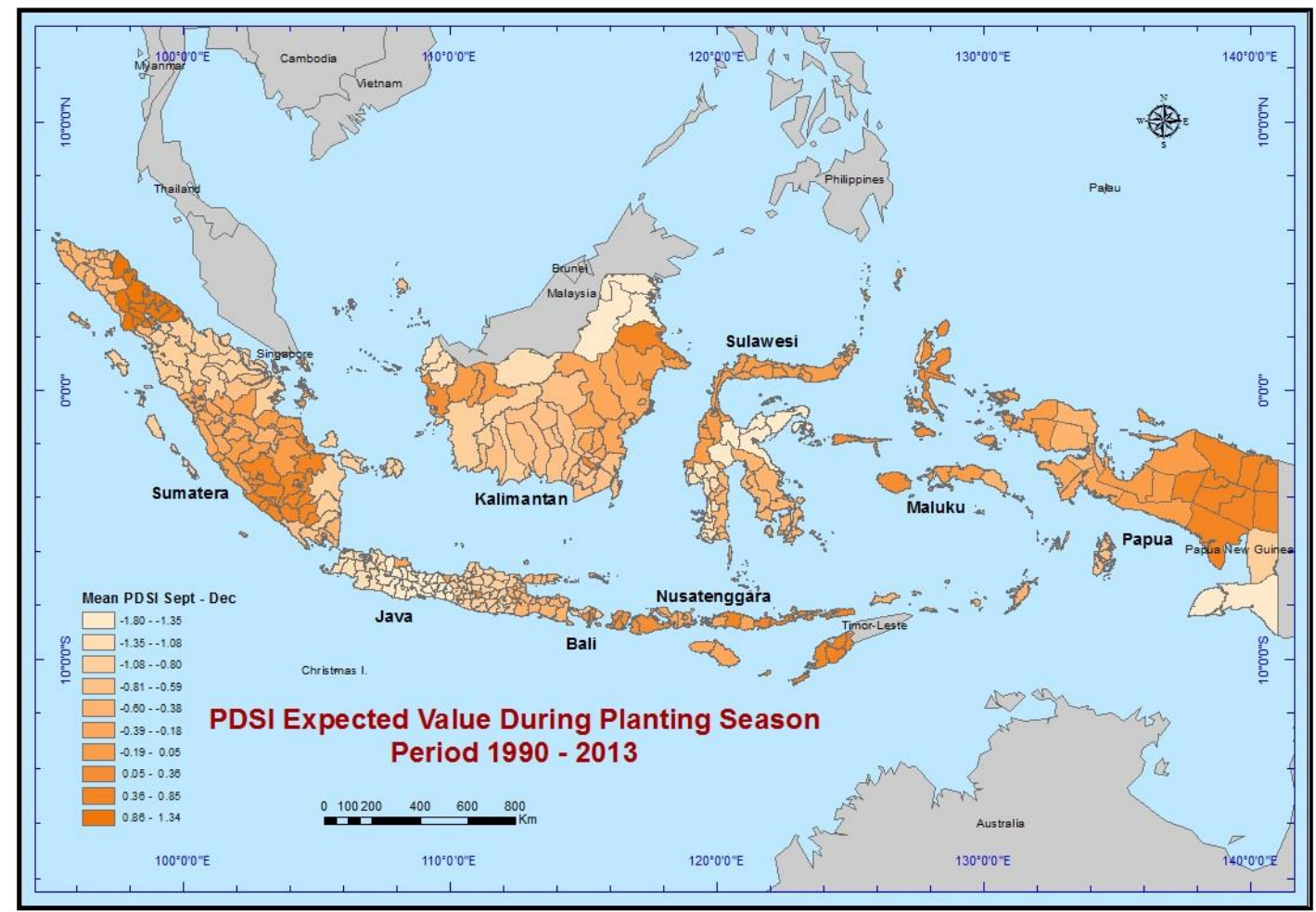

Note: The PDSI value in the figure was spotted in January 2013 from Dai's PDSI dataset. The self-calibrated PDSI dataset is provided by the NOAA/OAR/ESRL PSD Boulder, Colorado, USA and is available online at http://www.esrl.noaa.gov/psd/data/gridded/data.pdsi.htm. The figure is to show how this paper divides each district into gridded regions. 
Annex 5 Palmer Drought Severity Index classifications

\begin{tabular}{|l|l|}
\hline \multicolumn{1}{|c|}{ INDEX } & \multicolumn{1}{c|}{ INDICATION } \\
\hline More than 4.00 & Extremely wet \\
\hline Between 3.00 and 3.99 & Very wet \\
\hline Between 2.00 and 2.99 & Moderately wet \\
\hline Between 1.00 and 1.99 & Slightly wet \\
\hline Between 0.50 and 0.99 & Incipient wet spell \\
\hline Between 0.49 and -0.49 & Near normal \\
\hline Between -0.5 and -0.99 & Incipient dry spell \\
\hline Between -1.00 and -1.99 & Mild drought \\
\hline Between -2.00 and -2.99 & Moderate drought \\
\hline Between -3.00 and -3.99 & Severe drought \\
\hline Between -4.00 or less & Extreme drought \\
\hline
\end{tabular}

Annex 6 Monthly variance explained for Sept-Dec planting season for rice in Indonesia

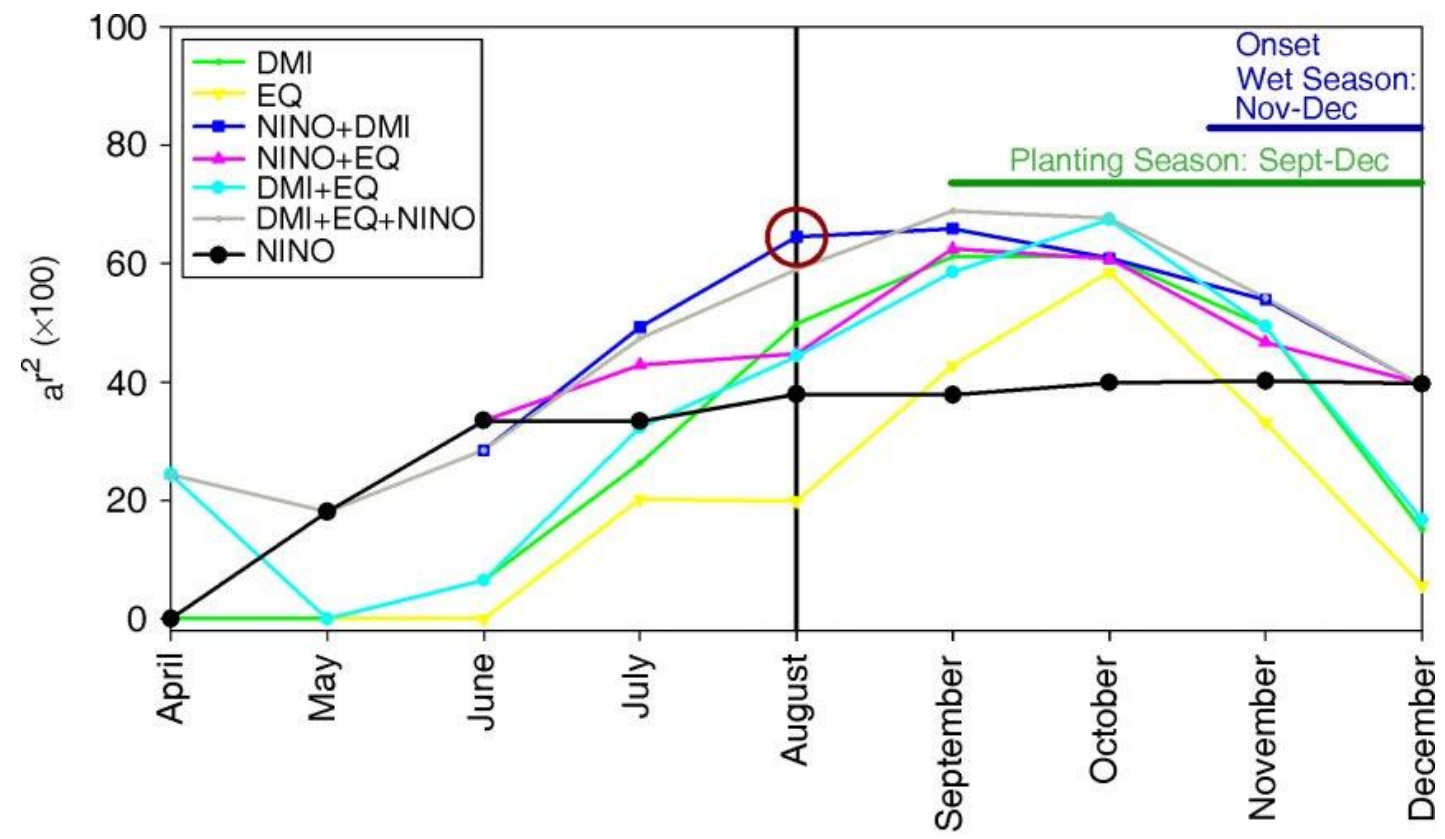

Note: sourced from D'Arrigo and Wilson (2008) 
Annex 7 Fixed bandwidth method illustration in selected districts in Indonesia

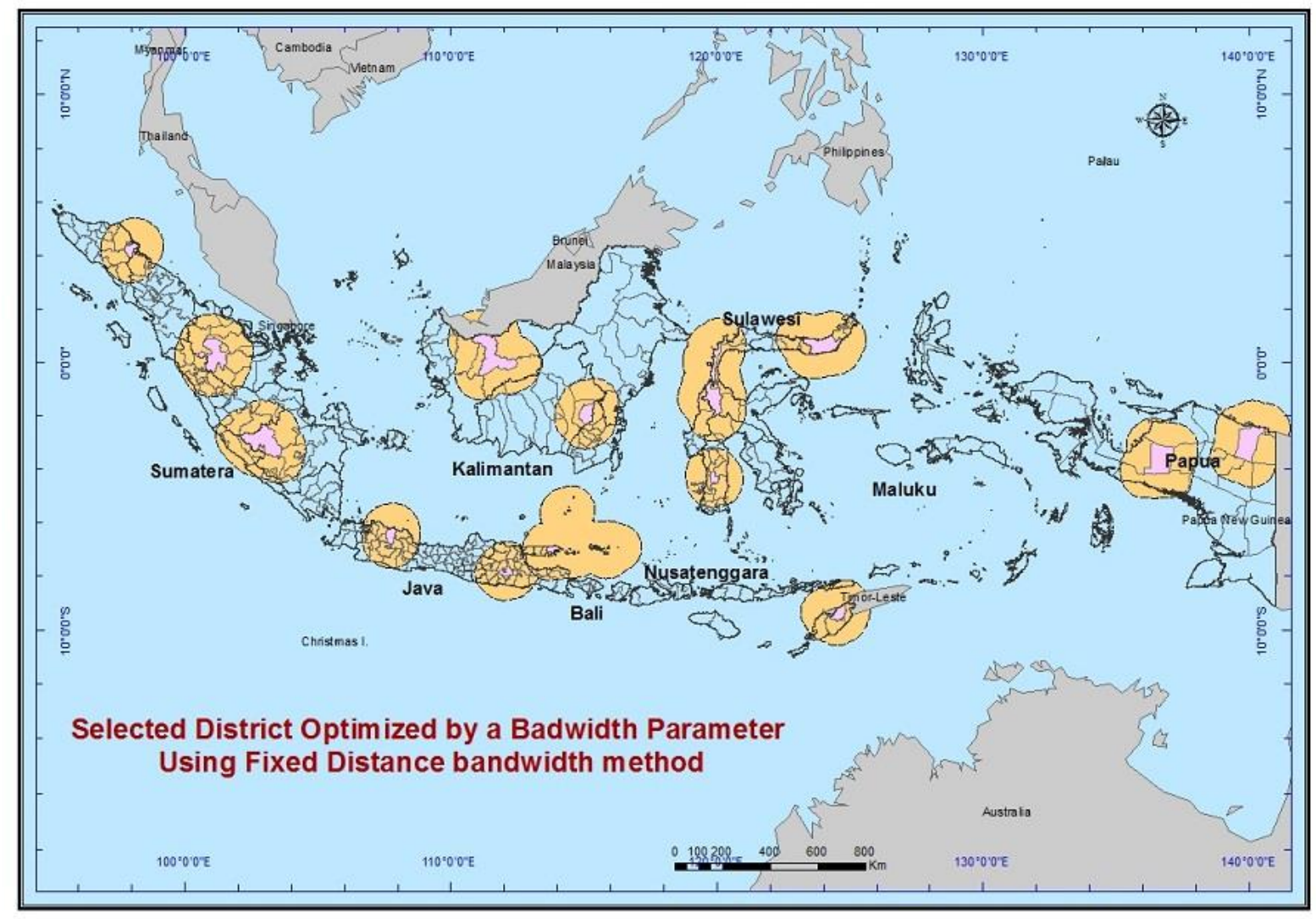

Annex 8 Fixed bandwidth method illustration in selected districts in Sumatera

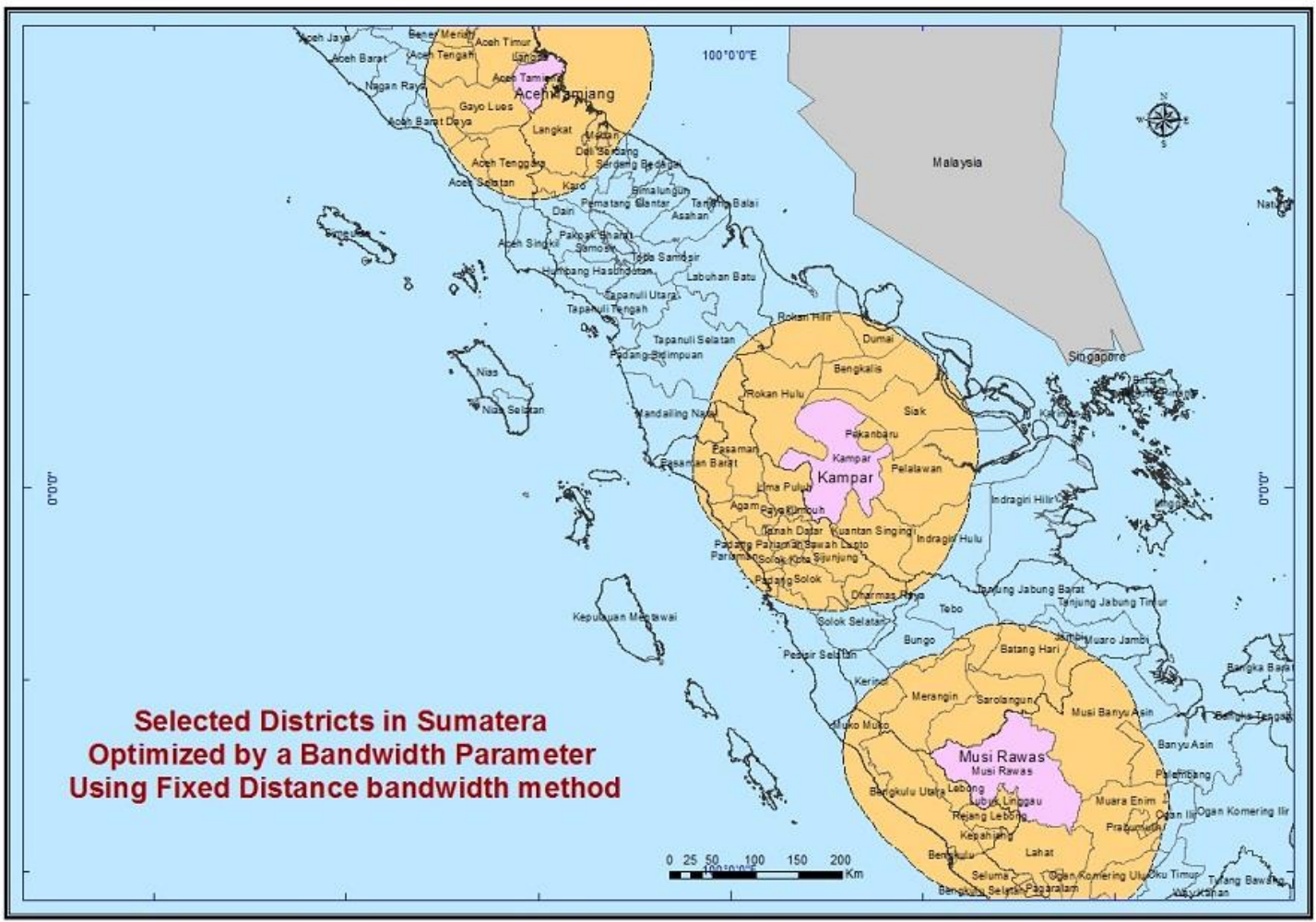


Annex 9 Spatial correlation between paddy yield and Indian Ocean Dipole (DMI) August at districts that are pseudo-significant at 5\% significance level

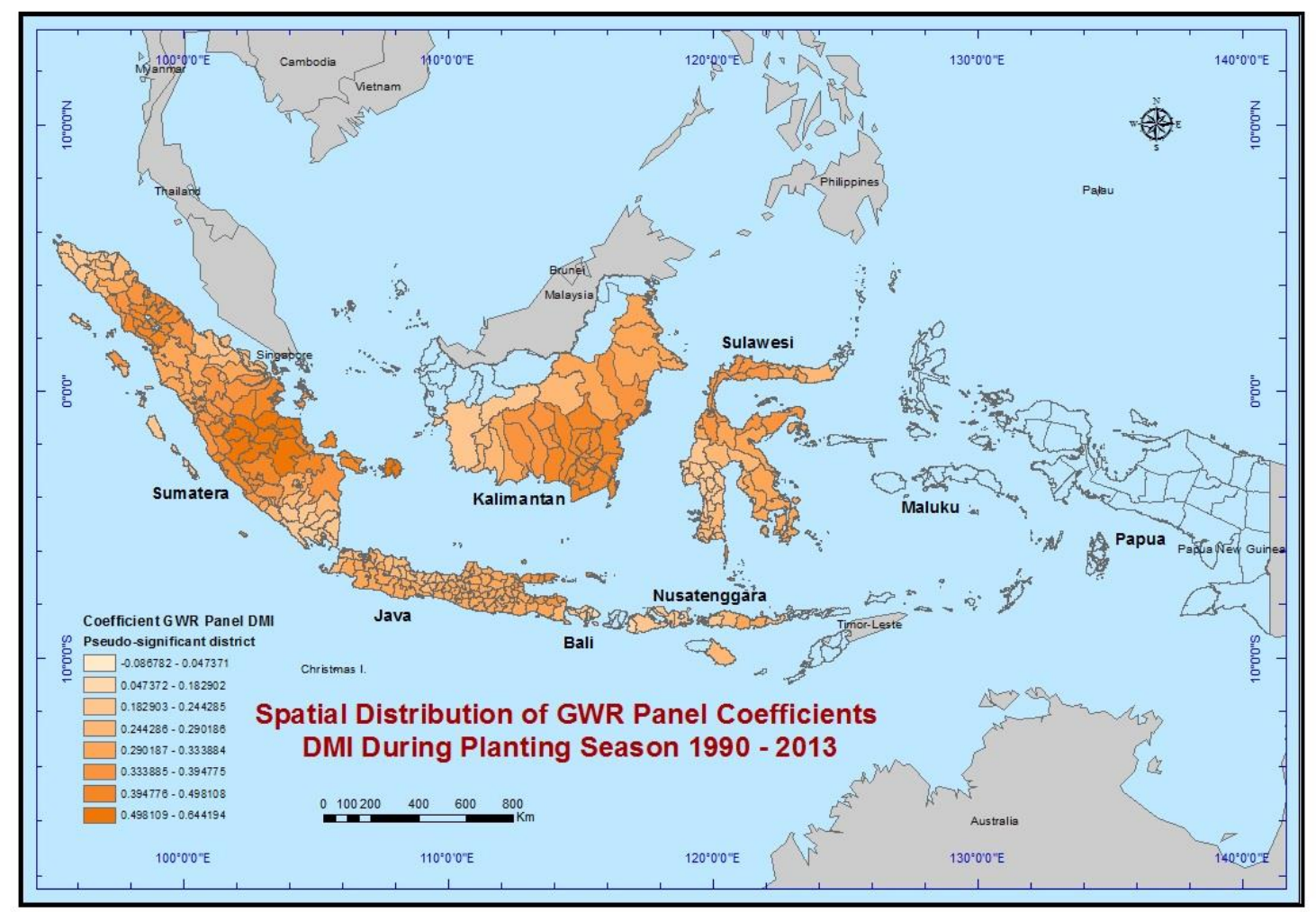

Annex 10 Spatial Correlation between Paddy Yield and El Niño (ENSO) August at Districts that are Pseudo-significant at 5\% Significance Level

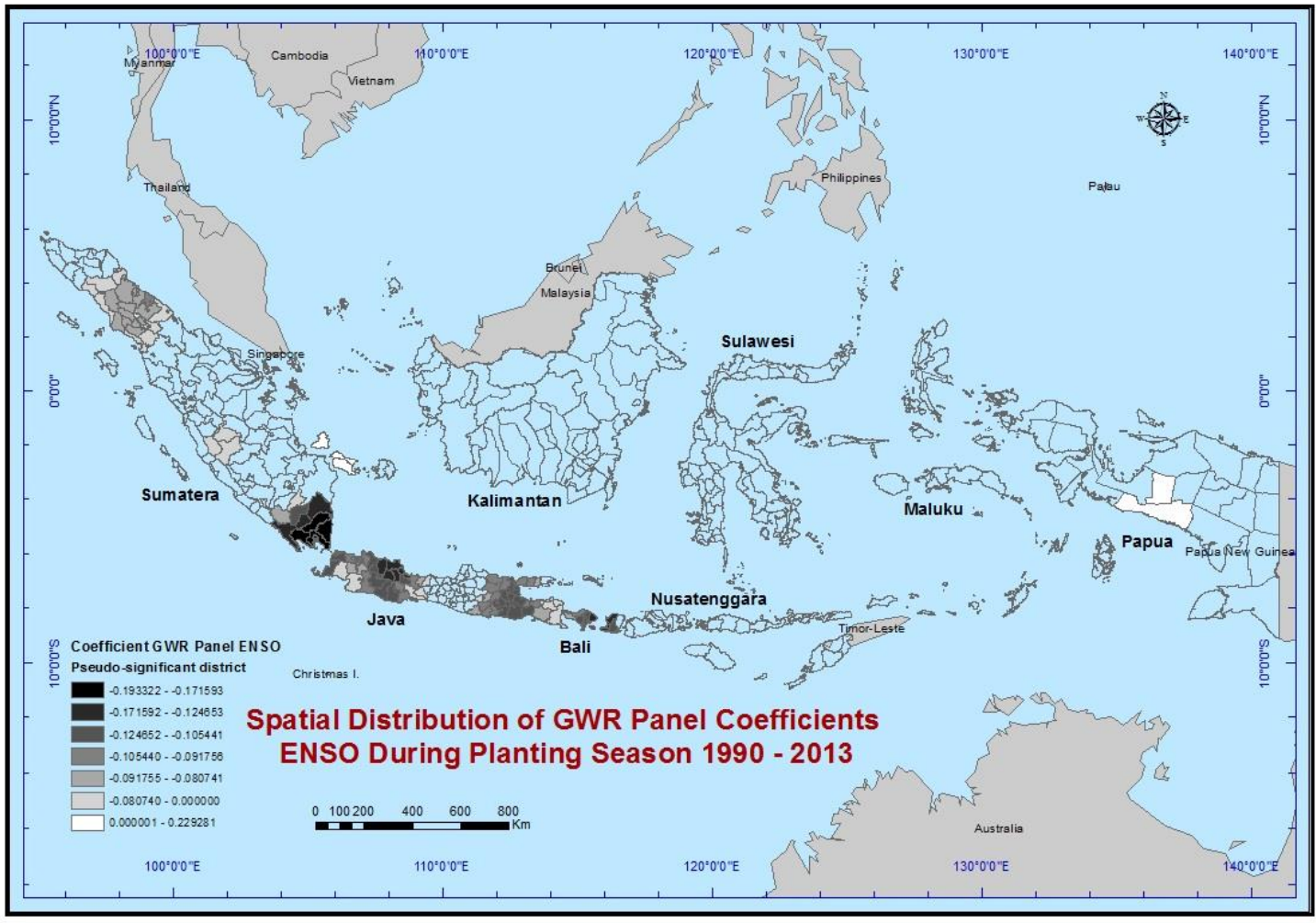


Annex 11 WGR panel results from ArcGIS

\begin{tabular}{|c|c|c|}
\hline Diagnostic & Value & DEFINITION \\
\hline Bandwidth & 152415.4 & $\begin{array}{l}\text { Fixed Distance bandwidth in meter (projected using UTM } \\
\text { coordinates) }\end{array}$ \\
\hline Sigma & 0.535317 & $\begin{array}{l}\text { This is the estimated standard deviation of the residuals, } \\
\text { square root of the normalized residual sum of squares } \\
\text { where the residual sum of squares is divided by the } \\
\text { effective degrees of freedom of the residual. }\end{array}$ \\
\hline $\mathrm{R} 2$ & 0.763329 & A measure of goodness of fit \\
\hline R2Adjusted & 0.753834 & A normalized measure of goodness of fit \\
\hline
\end{tabular}

Source: Definition is resourced from the ESRI's ArcGIS Desktop help

http://webhelp.esri.com/arcgisdesktop/9.3/index.cfm?TopicName=Interpreting_GWR_results

Annex 12 Table parameters of Weather Index Insurance (WII) using drought indices PDSI for rice production in major islands in Indonesia

\begin{tabular}{lllllll}
\hline \multicolumn{1}{c}{ Island } & $\begin{array}{l}\text { Insured } \\
\text { (IDR) }\end{array}$ & $\begin{array}{l}\text { Trigger } \\
\text { (PDSI) }\end{array}$ & $\begin{array}{l}\text { Exit } \\
\text { (PDSI) }\end{array}$ & Tick (IDR) & $\begin{array}{l}\text { Average } \\
\text { Payout (IDR) }\end{array}$ & $\begin{array}{l}\text { Premium } \\
\text { (IDR) }\end{array}$ \\
\hline Sumatera & $2,000,000$ & -2.020 & -6.907 & 409,249 & 14,161 & 14,000 \\
Kalimantan & $2,000,000$ & -2.402 & -6.265 & 517,732 & 21,874 & 20,832 \\
Sulawesi & $2,000,000$ & -2.832 & -7.583 & 420,964 & 26,951 & 25,668 \\
Papua & $2,000,000$ & -2.366 & -5.808 & 581,084 & 55,369 & 52,732 \\
\hline
\end{tabular}

Note: Triggers are determined by using Logistic regression models solved in Bayesian Estimation (Khalil, Kwon, Lall, Miranda, $\&$ Skees, 2007). Pexc=0.1. Exits are min. value PDSI of statistically significant districts

\section{Annex 13 GWR estimation using yield and time trend}

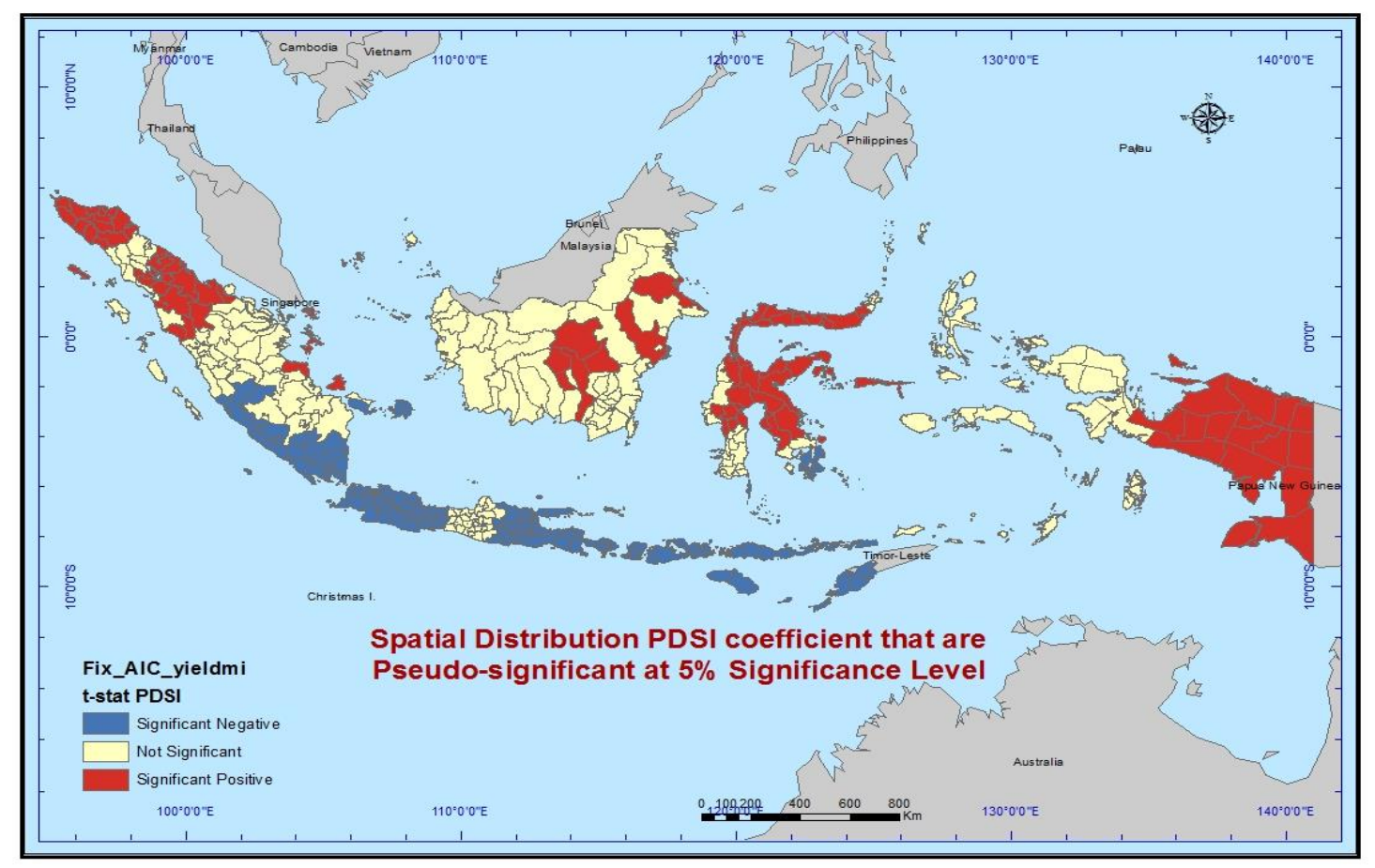


Annex 14 Parameters GWR panel for districts that are positively pseudo-significant at 5\% significance level

\begin{tabular}{|c|c|c|c|c|c|c|c|c|c|c|c|c|}
\hline No & District & Intercept & ß PDSI & ß ENSO & $ß \mathrm{ENSO}^{2}$ & ß DMI & Residual & SE & SE PDSI & SE ENSO & SE ENSO2 & SE PDSI \\
\hline 1 & Bangka Tengah & 2.814 & 0.123 & 0.229 & -0.099 & 0.437 & -0.532 & 0.499 & 0.021 & 0.076 & 0.056 & 0.110 \\
\hline 2 & Belitung Timur & 2.807 & 0.122 & 0.232 & -0.220 & 0.644 & 0.701 & 0.470 & 0.045 & 0.148 & 0.092 & 0.176 \\
\hline 3 & Belitung & 2.761 & 0.118 & 0.224 & -0.208 & 0.621 & -0.666 & 0.476 & 0.040 & 0.133 & 0.083 & 0.160 \\
\hline 4 & Bangka Selatan & 2.882 & 0.118 & 0.215 & -0.106 & 0.444 & -0.292 & 0.500 & 0.020 & 0.071 & 0.053 & 0.105 \\
\hline 5 & Bangka Barat & 3.078 & 0.118 & 0.193 & -0.076 & 0.461 & 0.221 & 0.502 & 0.018 & 0.063 & 0.048 & 0.094 \\
\hline 6 & Mimika & 2.853 & 0.103 & 0.213 & -0.010 & 0.166 & -0.705 & 0.489 & 0.025 & 0.101 & 0.079 & 0.159 \\
\hline 7 & Paniai & 2.857 & 0.086 & 0.172 & -0.006 & 0.123 & -0.754 & 0.501 & 0.021 & 0.088 & 0.068 & 0.138 \\
\hline 8 & Puncak Jaya & 2.869 & 0.086 & 0.119 & 0.020 & 0.093 & 0.377 & 0.502 & 0.019 & 0.075 & 0.063 & 0.125 \\
\hline 9 & Jayapura & 2.949 & 0.086 & 0.018 & 0.032 & 0.172 & -0.673 & 0.505 & 0.022 & 0.070 & 0.071 & 0.137 \\
\hline 10 & Tolikara & 2.928 & 0.081 & 0.048 & 0.032 & 0.134 & 0.334 & 0.507 & 0.019 & 0.069 & 0.064 & 0.125 \\
\hline 11 & Jayapura Kota & 2.997 & 0.081 & 0.011 & 0.018 & 0.183 & 0.248 & 0.495 & 0.029 & 0.089 & 0.092 & 0.175 \\
\hline 12 & Keerom & 2.955 & 0.079 & 0.012 & 0.025 & 0.169 & -0.134 & 0.505 & 0.025 & 0.077 & 0.079 & 0.151 \\
\hline 13 & Jayawijaya & 2.962 & 0.079 & 0.039 & 0.027 & 0.164 & -0.986 & 0.506 & 0.019 & 0.068 & 0.063 & 0.124 \\
\hline 14 & Sarmi & 2.908 & 0.078 & 0.040 & 0.031 & 0.127 & -0.096 & 0.495 & 0.022 & 0.075 & 0.072 & 0.140 \\
\hline 15 & Dompu & 4.250 & 0.075 & 0.011 & -0.053 & 0.232 & -0.424 & 0.501 & 0.038 & 0.071 & 0.058 & 0.109 \\
\hline 16 & Yahukimo & 2.960 & 0.072 & 0.028 & 0.023 & 0.157 & 0.243 & 0.501 & 0.019 & 0.068 & 0.065 & 0.126 \\
\hline 17 & Peg. Bintang & 2.875 & 0.070 & 0.024 & 0.027 & 0.125 & -0.262 & 0.494 & 0.022 & 0.076 & 0.077 & 0.146 \\
\hline 18 & Timor Tengah Selatan & 2.789 & 0.069 & 0.042 & -0.079 & 0.190 & -0.599 & 0.500 & 0.033 & 0.067 & 0.060 & 0.117 \\
\hline 19 & Waropen & 2.831 & 0.066 & 0.130 & -0.003 & 0.047 & -0.274 & 0.495 & 0.021 & 0.080 & 0.067 & 0.135 \\
\hline 20 & Buton & 3.419 & 0.066 & 0.030 & -0.066 & 0.297 & -0.557 & 0.509 & 0.020 & 0.075 & 0.057 & 0.109 \\
\hline 21 & $\mathrm{Bau}$ & 3.421 & 0.066 & 0.030 & -0.065 & 0.299 & 0.028 & 0.507 & 0.020 & 0.075 & 0.057 & 0.109 \\
\hline 22 & Banggai & 3.882 & 0.065 & 0.090 & -0.073 & 0.373 & -0.809 & 0.505 & 0.015 & 0.073 & 0.055 & 0.105 \\
\hline 23 & Timor Tengah Utara & 2.694 & 0.065 & 0.041 & -0.085 & 0.195 & -0.554 & 0.500 & 0.033 & 0.066 & 0.059 & 0.116 \\
\hline
\end{tabular}




\begin{tabular}{|c|c|c|c|c|c|c|c|c|c|c|c|c|}
\hline 24 & Seruyan & 2.301 & 0.064 & 0.021 & -0.044 & 0.307 & -0.156 & 0.507 & 0.028 & 0.066 & 0.054 & 0.102 \\
\hline 25 & Kupang & 2.861 & 0.064 & 0.027 & -0.058 & 0.172 & -0.213 & 0.499 & 0.032 & 0.066 & 0.058 & 0.114 \\
\hline 26 & Kota Waringin Timur & 2.368 & 0.063 & 0.008 & -0.042 & 0.336 & -0.050 & 0.514 & 0.023 & 0.057 & 0.048 & 0.092 \\
\hline 27 & Banyu Asin & 3.411 & 0.063 & 0.050 & -0.088 & 0.443 & 0.663 & 0.517 & 0.013 & 0.047 & 0.036 & 0.071 \\
\hline 28 & Muna & 3.489 & 0.063 & 0.027 & -0.068 & 0.296 & -1.707 & 0.512 & 0.019 & 0.072 & 0.053 & 0.101 \\
\hline 29 & Asmat & 3.090 & 0.063 & 0.042 & 0.017 & 0.193 & 0.386 & 0.484 & 0.023 & 0.086 & 0.082 & 0.159 \\
\hline 30 & Kota Waringin Barat & 2.265 & 0.062 & 0.028 & -0.048 & 0.286 & -0.522 & 0.512 & 0.030 & 0.072 & 0.058 & 0.111 \\
\hline 31 & Tojo Una-una & 3.921 & 0.062 & 0.086 & -0.074 & 0.345 & -0.481 & 0.510 & 0.013 & 0.062 & 0.047 & 0.088 \\
\hline 32 & Wakatobi & 3.291 & 0.062 & 0.031 & -0.053 & 0.262 & 0.098 & 0.478 & 0.024 & 0.090 & 0.073 & 0.138 \\
\hline 33 & Lamandau & 2.276 & 0.059 & 0.038 & -0.044 & 0.252 & -0.267 & 0.505 & 0.024 & 0.065 & 0.054 & 0.103 \\
\hline 34 & Katingan & 2.387 & 0.059 & -0.002 & -0.045 & 0.353 & 0.745 & 0.515 & 0.020 & 0.054 & 0.046 & 0.087 \\
\hline 35 & Kutai Barat & 2.808 & 0.059 & 0.071 & -0.025 & 0.296 & 0.093 & 0.500 & 0.020 & 0.076 & 0.057 & 0.107 \\
\hline 36 & Nabire & 3.059 & 0.058 & 0.099 & -0.010 & 0.180 & 0.647 & 0.486 & 0.023 & 0.101 & 0.070 & 0.141 \\
\hline 37 & Ketapang & 2.380 & 0.057 & 0.056 & -0.048 & 0.226 & 0.029 & 0.492 & 0.023 & 0.071 & 0.060 & 0.114 \\
\hline 38 & Murung Raya & 2.301 & 0.056 & 0.047 & -0.032 & 0.276 & -0.289 & 0.496 & 0.020 & 0.070 & 0.056 & 0.105 \\
\hline 39 & Majene & 4.520 & 0.056 & 0.072 & -0.071 & 0.224 & -0.351 & 0.520 & 0.014 & 0.054 & 0.039 & 0.073 \\
\hline 40 & Polewali Mandar & 4.546 & 0.056 & 0.075 & -0.074 & 0.225 & 0.400 & 0.523 & 0.013 & 0.051 & 0.037 & 0.069 \\
\hline 41 & Gorontalo Kota & 4.399 & 0.056 & 0.061 & -0.033 & 0.310 & 0.428 & 0.517 & 0.015 & 0.072 & 0.052 & 0.099 \\
\hline 42 & Rokan Hilir & 3.668 & 0.055 & 0.020 & -0.017 & 0.252 & -0.391 & 0.519 & 0.010 & 0.040 & 0.038 & 0.069 \\
\hline 43 & Kapuas Hulu & 2.195 & 0.055 & 0.050 & -0.060 & 0.196 & -0.378 & 0.481 & 0.027 & 0.084 & 0.078 & 0.146 \\
\hline 44 & Bone Bolango & 4.385 & 0.055 & 0.063 & -0.032 & 0.299 & 0.257 & 0.517 & 0.015 & 0.071 & 0.051 & 0.097 \\
\hline 45 & Palangkaraya & 2.597 & 0.055 & 0.003 & -0.069 & 0.392 & -1.448 & 0.519 & 0.015 & 0.049 & 0.040 & 0.075 \\
\hline 46 & Mamasa & 4.494 & 0.055 & 0.072 & -0.069 & 0.229 & -0.225 & 0.523 & 0.013 & 0.051 & 0.037 & 0.070 \\
\hline 47 & Poso & 4.136 & 0.054 & 0.068 & -0.070 & 0.306 & -1.332 & 0.519 & 0.013 & 0.055 & 0.040 & 0.076 \\
\hline 48 & Boven Digoel & 2.673 & 0.054 & 0.001 & -0.038 & 0.311 & -0.272 & 0.460 & 0.025 & 0.104 & 0.100 & 0.190 \\
\hline
\end{tabular}




\begin{tabular}{|c|c|c|c|c|c|c|c|c|c|c|c|c|}
\hline 49 & Siak & 3.501 & 0.054 & 0.003 & -0.035 & 0.320 & 0.059 & 0.522 & 0.010 & 0.041 & 0.038 & 0.069 \\
\hline 50 & Pekanbaru & 3.732 & 0.054 & 0.003 & -0.037 & 0.329 & -2.161 & 0.525 & 0.009 & 0.036 & 0.033 & 0.060 \\
\hline 51 & Tana Toraja & 4.479 & 0.053 & 0.072 & -0.069 & 0.236 & -0.776 & 0.525 & 0.012 & 0.048 & 0.035 & 0.066 \\
\hline 52 & Dumai & 3.392 & 0.053 & 0.023 & -0.022 & 0.236 & -0.815 & 0.516 & 0.012 & 0.047 & 0.045 & 0.081 \\
\hline 53 & Pinrang & 4.572 & 0.053 & 0.072 & -0.076 & 0.232 & 0.025 & 0.526 & 0.012 & 0.047 & 0.034 & 0.064 \\
\hline 54 & Melawi & 2.225 & 0.053 & 0.020 & -0.044 & 0.223 & -0.162 & 0.500 & 0.020 & 0.059 & 0.052 & 0.099 \\
\hline 55 & Palu & 4.145 & 0.053 & 0.067 & -0.087 & 0.339 & 0.082 & 0.511 & 0.015 & 0.069 & 0.052 & 0.098 \\
\hline 56 & Donggala & 4.148 & 0.053 & 0.066 & -0.086 & 0.336 & -0.755 & 0.512 & 0.015 & 0.068 & 0.051 & 0.097 \\
\hline 57 & Konawe Selatan & 3.651 & 0.053 & 0.017 & -0.065 & 0.303 & 0.223 & 0.513 & 0.017 & 0.066 & 0.049 & 0.093 \\
\hline 58 & Gunung Mas & 2.322 & 0.052 & 0.003 & -0.049 & 0.336 & -0.361 & 0.512 & 0.019 & 0.057 & 0.045 & 0.085 \\
\hline 59 & Barito Utara & 2.819 & 0.052 & 0.032 & -0.077 & 0.400 & -1.091 & 0.519 & 0.014 & 0.051 & 0.039 & 0.073 \\
\hline 60 & Luwu Utara & 4.328 & 0.052 & 0.065 & -0.063 & 0.259 & 0.159 & 0.524 & 0.012 & 0.049 & 0.036 & 0.067 \\
\hline 61 & Bolaang Mongondow & 4.283 & 0.052 & 0.069 & -0.024 & 0.247 & -0.833 & 0.517 & 0.015 & 0.069 & 0.049 & 0.094 \\
\hline 62 & Enrekang & 4.537 & 0.052 & 0.069 & -0.073 & 0.236 & -0.838 & 0.526 & 0.012 & 0.046 & 0.033 & 0.063 \\
\hline 63 & Palopo & 4.432 & 0.051 & 0.067 & -0.066 & 0.243 & 0.409 & 0.523 & 0.012 & 0.047 & 0.034 & 0.064 \\
\hline 64 & Konawe & 3.654 & 0.051 & 0.017 & -0.065 & 0.305 & 0.595 & 0.510 & 0.017 & 0.067 & 0.050 & 0.096 \\
\hline 65 & Mamuju & 4.393 & 0.051 & 0.060 & -0.061 & 0.244 & 0.378 & 0.520 & 0.013 & 0.053 & 0.038 & 0.072 \\
\hline 66 & Morowali & 3.966 & 0.051 & 0.054 & -0.062 & 0.307 & -0.109 & 0.515 & 0.014 & 0.059 & 0.044 & 0.083 \\
\hline 67 & Pare-pare & 4.611 & 0.051 & 0.067 & -0.078 & 0.235 & -0.819 & 0.526 & 0.012 & 0.045 & 0.033 & 0.062 \\
\hline 68 & Pulang Pisau & 2.888 & 0.050 & -0.004 & -0.083 & 0.416 & -0.002 & 0.521 & 0.015 & 0.047 & 0.036 & 0.069 \\
\hline 69 & Luwu & 4.457 & 0.050 & 0.065 & -0.068 & 0.242 & -0.931 & 0.523 & 0.012 & 0.046 & 0.033 & 0.063 \\
\hline 70 & Tanjung Jabung Timur & 3.660 & 0.050 & -0.009 & -0.175 & 0.566 & -0.360 & 0.516 & 0.015 & 0.053 & 0.043 & 0.081 \\
\hline 71 & Kapuas & 2.786 & 0.050 & 0.003 & -0.085 & 0.418 & -0.544 & 0.522 & 0.014 & 0.046 & 0.036 & 0.069 \\
\hline 72 & Bengkalis & 3.320 & 0.050 & 0.004 & -0.033 & 0.285 & -0.323 & 0.517 & 0.012 & 0.049 & 0.045 & 0.082 \\
\hline 73 & Sidenreng Rappang & 4.562 & 0.049 & 0.063 & -0.074 & 0.238 & 1.047 & 0.525 & 0.012 & 0.045 & 0.032 & 0.061 \\
\hline
\end{tabular}




\begin{tabular}{|c|c|c|c|c|c|c|c|c|c|c|c|c|}
\hline 74 & Luwu Timur & 4.143 & 0.049 & 0.054 & -0.059 & 0.279 & 0.554 & 0.515 & 0.012 & 0.051 & 0.038 & 0.071 \\
\hline 75 & Pelalawan & 3.616 & 0.049 & -0.018 & -0.057 & 0.382 & -0.432 & 0.522 & 0.010 & 0.039 & 0.036 & 0.065 \\
\hline 76 & Barru & 4.636 & 0.048 & 0.061 & -0.081 & 0.241 & 0.623 & 0.527 & 0.012 & 0.046 & 0.034 & 0.063 \\
\hline 77 & Gorontalo & 4.412 & 0.048 & 0.036 & -0.032 & 0.320 & 0.175 & 0.516 & 0.015 & 0.074 & 0.053 & 0.101 \\
\hline 78 & Kampar & 3.955 & 0.048 & -0.006 & -0.043 & 0.337 & -1.314 & 0.527 & 0.008 & 0.033 & 0.031 & 0.057 \\
\hline 79 & Minahasa Selatan & 4.121 & 0.048 & 0.081 & -0.005 & 0.163 & 0.428 & 0.521 & 0.016 & 0.076 & 0.053 & 0.102 \\
\hline 80 & Pangkajene \& Kepulauan & 4.649 & 0.048 & 0.060 & -0.084 & 0.245 & 0.448 & 0.526 & 0.013 & 0.049 & 0.035 & 0.067 \\
\hline 81 & Mamuju Utara & 4.232 & 0.048 & 0.049 & -0.070 & 0.297 & 0.446 & 0.514 & 0.015 & 0.061 & 0.045 & 0.085 \\
\hline 82 & Takalar & 4.626 & 0.048 & 0.059 & -0.091 & 0.263 & 0.475 & 0.524 & 0.015 & 0.055 & 0.040 & 0.076 \\
\hline 83 & Jeneponto & 4.592 & 0.047 & 0.055 & -0.092 & 0.278 & -1.085 & 0.524 & 0.015 & 0.056 & 0.041 & 0.077 \\
\hline 84 & Makassar & 4.646 & 0.047 & 0.060 & -0.088 & 0.253 & -0.589 & 0.525 & 0.014 & 0.053 & 0.038 & 0.072 \\
\hline 85 & Muaro Jambi & 3.700 & 0.047 & -0.022 & -0.180 & 0.586 & 0.382 & 0.519 & 0.014 & 0.049 & 0.039 & 0.076 \\
\hline 86 & Soppeng & 4.614 & 0.047 & 0.058 & -0.078 & 0.243 & -0.115 & 0.527 & 0.012 & 0.045 & 0.033 & 0.062 \\
\hline 87 & Penajam Paser Utara & 3.389 & 0.047 & 0.043 & -0.095 & 0.428 & 1.423 & 0.516 & 0.016 & 0.065 & 0.046 & 0.088 \\
\hline 88 & Boalemo & 4.376 & 0.047 & 0.033 & -0.039 & 0.334 & 0.160 & 0.516 & 0.015 & 0.073 & 0.053 & 0.101 \\
\hline 89 & Gowa & 4.617 & 0.047 & 0.056 & -0.088 & 0.266 & 0.194 & 0.526 & 0.014 & 0.054 & 0.039 & 0.074 \\
\hline 90 & Palembang & 3.470 & 0.047 & 0.021 & -0.075 & 0.382 & -0.372 & 0.520 & 0.013 & 0.045 & 0.035 & 0.069 \\
\hline 91 & Maros & 4.635 & 0.047 & 0.057 & -0.085 & 0.255 & 0.469 & 0.526 & 0.013 & 0.051 & 0.037 & 0.069 \\
\hline 92 & Bantaeng & 4.574 & 0.047 & 0.051 & -0.089 & 0.283 & 0.406 & 0.523 & 0.014 & 0.054 & 0.040 & 0.075 \\
\hline 93 & Minahasa & 4.060 & 0.046 & 0.085 & 0.002 & 0.133 & -0.414 & 0.521 & 0.017 & 0.079 & 0.055 & 0.107 \\
\hline 94 & Wajo & 4.554 & 0.046 & 0.054 & -0.073 & 0.244 & -0.446 & 0.524 & 0.011 & 0.044 & 0.032 & 0.060 \\
\hline 95 & Tomohon & 4.054 & 0.046 & 0.085 & 0.003 & 0.130 & 0.433 & 0.521 & 0.017 & 0.079 & 0.055 & 0.107 \\
\hline 96 & Ogan Komering Ilir & 3.497 & 0.046 & 0.023 & -0.078 & 0.359 & -0.291 & 0.518 & 0.013 & 0.046 & 0.036 & 0.072 \\
\hline 97 & Parigi Moutong & 4.163 & 0.046 & 0.048 & -0.089 & 0.362 & 0.711 & 0.508 & 0.016 & 0.072 & 0.053 & 0.101 \\
\hline 98 & Rokan Hulu & 3.931 & 0.046 & 0.000 & -0.037 & 0.292 & -1.482 & 0.525 & 0.008 & 0.034 & 0.032 & 0.058 \\
\hline
\end{tabular}




\begin{tabular}{|c|c|c|c|c|c|c|c|c|c|c|c|c|}
\hline 99 & Barito Selatan & 2.956 & 0.046 & -0.002 & -0.094 & 0.437 & -0.962 & 0.524 & 0.014 & 0.047 & 0.036 & 0.068 \\
\hline 100 & Balikpapan & 3.455 & 0.046 & 0.045 & -0.100 & 0.426 & -1.118 & 0.513 & 0.018 & 0.073 & 0.051 & 0.095 \\
\hline 101 & Bulukumba & 4.544 & 0.045 & 0.046 & -0.087 & 0.289 & -0.598 & 0.522 & 0.014 & 0.053 & 0.039 & 0.073 \\
\hline 102 & Sumenep & 5.141 & 0.045 & -0.059 & -0.122 & 0.458 & -0.955 & 0.517 & 0.020 & 0.041 & 0.033 & 0.065 \\
\hline 103 & Manado & 4.026 & 0.045 & 0.085 & 0.007 & 0.116 & -0.497 & 0.521 & 0.017 & 0.080 & 0.056 & 0.108 \\
\hline 104 & Sinjai & 4.579 & 0.045 & 0.048 & -0.084 & 0.274 & -0.961 & 0.524 & 0.013 & 0.051 & 0.037 & 0.070 \\
\hline 105 & Sintang & 2.310 & 0.045 & 0.029 & -0.064 & 0.170 & -0.812 & 0.506 & 0.021 & 0.070 & 0.061 & 0.114 \\
\hline 106 & Minahasa Utara & 4.003 & 0.045 & 0.087 & 0.009 & 0.107 & 0.013 & 0.518 & 0.017 & 0.081 & 0.057 & 0.110 \\
\hline 107 & Bitung & 3.993 & 0.045 & 0.088 & 0.009 & 0.104 & -1.433 & 0.517 & 0.017 & 0.082 & 0.057 & 0.111 \\
\hline 108 & Bombana & 3.826 & 0.044 & 0.001 & -0.064 & 0.304 & 0.217 & 0.511 & 0.015 & 0.056 & 0.041 & 0.078 \\
\hline 109 & Musi Banyu Asin & 3.600 & 0.044 & -0.012 & -0.129 & 0.504 & -0.403 & 0.520 & 0.012 & 0.041 & 0.032 & 0.064 \\
\hline 110 & Bone & 4.596 & 0.044 & 0.049 & -0.078 & 0.255 & 0.293 & 0.525 & 0.012 & 0.047 & 0.034 & 0.064 \\
\hline 111 & Banjarmasin & 3.173 & 0.044 & -0.019 & -0.089 & 0.431 & 0.153 & 0.522 & 0.016 & 0.052 & 0.040 & 0.076 \\
\hline 112 & Labuhan Batu & 4.010 & 0.044 & -0.032 & -0.037 & 0.353 & -0.174 & 0.522 & 0.009 & 0.037 & 0.035 & 0.064 \\
\hline 113 & Barito Timur & 3.043 & 0.044 & -0.007 & -0.096 & 0.445 & -0.246 & 0.526 & 0.014 & 0.048 & 0.036 & 0.068 \\
\hline 114 & Barito Kuala & 3.152 & 0.043 & -0.020 & -0.091 & 0.436 & -0.785 & 0.524 & 0.015 & 0.049 & 0.038 & 0.072 \\
\hline 115 & Paser & 3.249 & 0.043 & 0.006 & -0.097 & 0.451 & -1.110 & 0.522 & 0.014 & 0.053 & 0.039 & 0.074 \\
\hline 116 & Tanah Laut & 3.240 & 0.043 & -0.022 & -0.085 & 0.427 & -0.387 & 0.518 & 0.017 & 0.057 & 0.044 & 0.084 \\
\hline 117 & Jambi & 3.737 & 0.043 & -0.034 & -0.184 & 0.588 & -1.180 & 0.520 & 0.013 & 0.047 & 0.039 & 0.074 \\
\hline 118 & Banjarbaru & 3.213 & 0.043 & -0.022 & -0.088 & 0.433 & 0.111 & 0.522 & 0.016 & 0.053 & 0.041 & 0.078 \\
\hline 119 & Tabalong & 3.128 & 0.042 & -0.007 & -0.097 & 0.451 & -0.420 & 0.525 & 0.014 & 0.049 & 0.037 & 0.069 \\
\hline 120 & Banjar & 3.237 & 0.041 & -0.028 & -0.090 & 0.441 & -0.777 & 0.523 & 0.015 & 0.051 & 0.040 & 0.075 \\
\hline 121 & Hulu Sungai Utara & 3.149 & 0.041 & -0.021 & -0.097 & 0.451 & 0.736 & 0.527 & 0.014 & 0.047 & 0.036 & 0.068 \\
\hline 122 & Tapin & 3.198 & 0.041 & -0.026 & -0.094 & 0.447 & 0.109 & 0.525 & 0.014 & 0.048 & 0.037 & 0.070 \\
\hline 123 & Kendari & 3.859 & 0.041 & 0.009 & -0.059 & 0.305 & -0.605 & 0.509 & 0.015 & 0.059 & 0.044 & 0.084 \\
\hline
\end{tabular}




\begin{tabular}{|c|c|c|c|c|c|c|c|c|c|c|c|c|}
\hline 124 & Kolaka Utara & 4.227 & 0.040 & 0.030 & -0.059 & 0.270 & -0.377 & 0.517 & 0.012 & 0.047 & 0.034 & 0.065 \\
\hline 125 & Lebong & 3.741 & 0.040 & -0.033 & -0.107 & 0.498 & 0.575 & 0.524 & 0.012 & 0.042 & 0.035 & 0.069 \\
\hline 126 & Hulu Sungai Selatan & 3.200 & 0.040 & -0.027 & -0.095 & 0.452 & -1.286 & 0.525 & 0.014 & 0.048 & 0.037 & 0.069 \\
\hline 127 & Bengkulu Utara & 3.747 & 0.040 & -0.031 & -0.101 & 0.488 & -0.846 & 0.522 & 0.013 & 0.045 & 0.037 & 0.074 \\
\hline 128 & Musi Rawas & 3.684 & 0.039 & -0.024 & -0.108 & 0.479 & -0.294 & 0.524 & 0.011 & 0.039 & 0.032 & 0.063 \\
\hline 129 & Balangan & 3.216 & 0.039 & -0.023 & -0.097 & 0.457 & 0.569 & 0.526 & 0.014 & 0.049 & 0.037 & 0.070 \\
\hline 130 & Indragiri Hulu & 3.860 & 0.039 & -0.046 & -0.099 & 0.447 & -0.535 & 0.522 & 0.009 & 0.036 & 0.032 & 0.060 \\
\hline 131 & Hulu Sungai Tengah & 3.219 & 0.039 & -0.028 & -0.096 & 0.455 & 0.478 & 0.524 & 0.014 & 0.048 & 0.037 & 0.070 \\
\hline 132 & Lubuk Linggau & 3.677 & 0.039 & -0.016 & -0.094 & 0.447 & -0.084 & 0.525 & 0.012 & 0.041 & 0.033 & 0.066 \\
\hline 133 & Rejang Lebong & 3.687 & 0.038 & -0.013 & -0.088 & 0.436 & -0.039 & 0.525 & 0.012 & 0.043 & 0.035 & 0.069 \\
\hline 134 & Batang Hari & 3.805 & 0.038 & -0.056 & -0.181 & 0.590 & 1.951 & 0.522 & 0.012 & 0.042 & 0.035 & 0.067 \\
\hline 135 & Tanjung Jabung Barat & 3.802 & 0.038 & -0.051 & -0.173 & 0.557 & -0.578 & 0.521 & 0.012 & 0.044 & 0.038 & 0.071 \\
\hline 136 & Sarolangun & 3.803 & 0.038 & -0.058 & -0.151 & 0.565 & -0.959 & 0.523 & 0.011 & 0.038 & 0.032 & 0.062 \\
\hline 137 & Tanah Bumbu & 3.295 & 0.038 & -0.037 & -0.087 & 0.447 & 0.441 & 0.520 & 0.016 & 0.054 & 0.041 & 0.077 \\
\hline 138 & Kuantan Singingi & 4.077 & 0.038 & -0.038 & -0.066 & 0.387 & -0.493 & 0.527 & 0.008 & 0.033 & 0.030 & 0.056 \\
\hline 139 & Bengkulu & 3.704 & 0.038 & -0.008 & -0.083 & 0.422 & -0.525 & 0.522 & 0.014 & 0.047 & 0.038 & 0.077 \\
\hline 140 & Lima Puluh & 4.193 & 0.038 & -0.021 & -0.051 & 0.334 & 0.723 & 0.528 & 0.008 & 0.034 & 0.032 & 0.059 \\
\hline 141 & Pasaman & 4.174 & 0.037 & -0.015 & -0.047 & 0.306 & 0.071 & 0.527 & 0.009 & 0.034 & 0.033 & 0.060 \\
\hline 142 & Kepahiang & 3.688 & 0.037 & -0.009 & -0.082 & 0.415 & 0.414 & 0.524 & 0.013 & 0.044 & 0.036 & 0.071 \\
\hline 143 & Pohuwato & 4.291 & 0.037 & 0.007 & -0.053 & 0.354 & 0.354 & 0.514 & 0.016 & 0.076 & 0.055 & 0.104 \\
\hline 144 & Kepulauan Mentawai & 4.322 & 0.037 & 0.022 & -0.046 & 0.234 & -0.778 & 0.496 & 0.012 & 0.051 & 0.047 & 0.088 \\
\hline 145 & Indragiri Hilir & 3.642 & 0.036 & -0.039 & -0.116 & 0.447 & 0.834 & 0.517 & 0.012 & 0.046 & 0.040 & 0.073 \\
\hline 146 & Kepulauan Selayar & 4.024 & 0.036 & -0.026 & -0.105 & 0.443 & -2.114 & 0.490 & 0.017 & 0.063 & 0.049 & 0.093 \\
\hline 147 & Kota Baru & 3.314 & 0.036 & -0.040 & -0.090 & 0.455 & 0.069 & 0.517 & 0.015 & 0.053 & 0.040 & 0.076 \\
\hline 148 & Kolaka & 4.030 & 0.035 & 0.001 & -0.058 & 0.291 & -0.444 & 0.514 & 0.013 & 0.050 & 0.037 & 0.070 \\
\hline
\end{tabular}




\begin{tabular}{|c|c|c|c|c|c|c|c|c|c|c|c|c|}
\hline 149 & Payakumbuh & 4.242 & 0.034 & -0.029 & -0.056 & 0.341 & 0.307 & 0.529 & 0.009 & 0.034 & 0.032 & 0.059 \\
\hline 150 & Tebo & 3.961 & 0.034 & -0.071 & -0.143 & 0.527 & 0.084 & 0.522 & 0.010 & 0.037 & 0.032 & 0.061 \\
\hline 151 & Merangin & 3.942 & 0.034 & -0.075 & -0.141 & 0.542 & -0.127 & 0.525 & 0.011 & 0.038 & 0.033 & 0.063 \\
\hline 152 & Pasaman Barat & 4.259 & 0.033 & -0.020 & -0.049 & 0.300 & -0.151 & 0.524 & 0.009 & 0.036 & 0.034 & 0.062 \\
\hline 153 & Tapanuli Selatan & 4.050 & 0.032 & -0.037 & -0.051 & 0.329 & 0.010 & 0.523 & 0.009 & 0.036 & 0.034 & 0.062 \\
\hline 154 & Seluma & 3.701 & 0.032 & -0.006 & -0.075 & 0.371 & -0.104 & 0.523 & 0.013 & 0.046 & 0.037 & 0.075 \\
\hline 155 & Nias & 3.767 & 0.032 & -0.041 & -0.074 & 0.347 & -0.766 & 0.501 & 0.013 & 0.050 & 0.048 & 0.087 \\
\hline 156 & Sijunjung & 4.229 & 0.031 & -0.045 & -0.066 & 0.369 & -0.058 & 0.528 & 0.008 & 0.034 & 0.031 & 0.057 \\
\hline 157 & Bukit Tinggi & 4.296 & 0.031 & -0.031 & -0.057 & 0.333 & -0.167 & 0.529 & 0.009 & 0.035 & 0.033 & 0.061 \\
\hline 158 & Bungo & 4.052 & 0.031 & -0.077 & -0.129 & 0.504 & -1.611 & 0.524 & 0.010 & 0.036 & 0.032 & 0.060 \\
\hline 159 & Tanjung Balai & 4.125 & 0.031 & -0.069 & -0.049 & 0.399 & -0.182 & 0.525 & 0.009 & 0.038 & 0.036 & 0.067 \\
\hline 160 & Tanah Datar & 4.298 & 0.030 & -0.037 & -0.060 & 0.342 & 0.545 & 0.529 & 0.009 & 0.035 & 0.032 & 0.060 \\
\hline 161 & Agam & 4.320 & 0.030 & -0.031 & -0.057 & 0.326 & -0.062 & 0.528 & 0.009 & 0.036 & 0.033 & 0.062 \\
\hline 162 & Dharmas Raya & 4.164 & 0.030 & -0.063 & -0.091 & 0.419 & 0.336 & 0.526 & 0.009 & 0.034 & 0.031 & 0.058 \\
\hline 163 & Mandailing Natal & 4.151 & 0.029 & -0.029 & -0.051 & 0.300 & 0.310 & 0.522 & 0.009 & 0.037 & 0.034 & 0.063 \\
\hline 164 & Padang Panjang & 4.324 & 0.029 & -0.036 & -0.059 & 0.337 & 0.594 & 0.529 & 0.009 & 0.035 & 0.033 & 0.061 \\
\hline 165 & Sawah Lunto & 4.295 & 0.029 & -0.044 & -0.063 & 0.353 & 0.994 & 0.529 & 0.009 & 0.035 & 0.032 & 0.059 \\
\hline 166 & Nias Selatan & 3.967 & 0.029 & -0.025 & -0.058 & 0.287 & -0.237 & 0.496 & 0.014 & 0.054 & 0.051 & 0.094 \\
\hline 167 & Muko & 3.994 & 0.029 & -0.072 & -0.115 & 0.479 & -0.347 & 0.521 & 0.011 & 0.042 & 0.037 & 0.070 \\
\hline 168 & Lahat & 3.673 & 0.028 & -0.012 & -0.070 & 0.344 & 0.016 & 0.525 & 0.012 & 0.041 & 0.033 & 0.066 \\
\hline 169 & Kerinci & 4.121 & 0.027 & -0.079 & -0.116 & 0.467 & 0.802 & 0.523 & 0.010 & 0.038 & 0.034 & 0.064 \\
\hline 170 & Asahan & 4.127 & 0.027 & -0.078 & -0.057 & 0.412 & -0.253 & 0.527 & 0.009 & 0.037 & 0.035 & 0.064 \\
\hline 171 & Solok Kota & 4.341 & 0.027 & -0.045 & -0.064 & 0.346 & -0.376 & 0.529 & 0.009 & 0.035 & 0.033 & 0.060 \\
\hline 172 & Padang Pariaman & 4.368 & 0.026 & -0.039 & -0.061 & 0.332 & 0.450 & 0.528 & 0.009 & 0.036 & 0.034 & 0.062 \\
\hline 173 & Ogan Ilir & 3.582 & 0.026 & -0.019 & -0.071 & 0.328 & 0.182 & 0.522 & 0.012 & 0.042 & 0.033 & 0.066 \\
\hline
\end{tabular}




\begin{tabular}{|c|c|c|c|c|c|c|c|c|c|c|c|c|}
\hline 174 & Toba Samosir & 4.084 & 0.026 & -0.076 & -0.064 & 0.417 & 0.548 & 0.526 & 0.009 & 0.035 & 0.033 & 0.061 \\
\hline 175 & Padang Sidimpuan & 4.076 & 0.026 & -0.050 & -0.061 & 0.347 & 0.338 & 0.523 & 0.009 & 0.037 & 0.034 & 0.063 \\
\hline 176 & Solok & 4.327 & 0.026 & -0.051 & -0.069 & 0.357 & 0.505 & 0.528 & 0.009 & 0.035 & 0.032 & 0.060 \\
\hline 177 & Pariaman & 4.380 & 0.026 & -0.038 & -0.061 & 0.328 & 0.154 & 0.528 & 0.009 & 0.036 & 0.034 & 0.063 \\
\hline 178 & Solok Selatan & 4.242 & 0.026 & -0.067 & -0.090 & 0.405 & 0.754 & 0.526 & 0.009 & 0.035 & 0.032 & 0.059 \\
\hline 179 & Tapanuli Utara & 4.056 & 0.025 & -0.073 & -0.069 & 0.410 & -0.185 & 0.525 & 0.009 & 0.035 & 0.033 & 0.061 \\
\hline 180 & Prabumulih & 3.607 & 0.024 & -0.023 & -0.069 & 0.324 & -1.000 & 0.524 & 0.011 & 0.040 & 0.032 & 0.063 \\
\hline 181 & Muara Enim & 3.623 & 0.024 & -0.022 & -0.068 & 0.323 & -0.873 & 0.524 & 0.011 & 0.039 & 0.031 & 0.062 \\
\hline 182 & Padang & 4.386 & 0.024 & -0.048 & -0.066 & 0.339 & 0.441 & 0.528 & 0.009 & 0.036 & 0.033 & 0.062 \\
\hline 183 & Pesisir Selatan & 4.300 & 0.023 & -0.065 & -0.086 & 0.382 & 0.565 & 0.524 & 0.010 & 0.036 & 0.033 & 0.062 \\
\hline 184 & Aceh Tamiang & 4.088 & 0.022 & -0.073 & -0.049 & 0.308 & -0.081 & 0.527 & 0.009 & 0.034 & 0.033 & 0.061 \\
\hline 185 & Pematang Siantar & 4.113 & 0.021 & -0.090 & -0.067 & 0.426 & -1.319 & 0.529 & 0.009 & 0.035 & 0.033 & 0.061 \\
\hline 186 & Aceh Singkil & 3.936 & 0.021 & -0.083 & -0.077 & 0.410 & -0.510 & 0.526 & 0.009 & 0.034 & 0.033 & 0.061 \\
\hline 187 & Samosir & 4.044 & 0.021 & -0.089 & -0.074 & 0.433 & 0.532 & 0.529 & 0.009 & 0.034 & 0.033 & 0.060 \\
\hline 188 & Langsa & 4.092 & 0.021 & -0.070 & -0.046 & 0.289 & -0.171 & 0.526 & 0.010 & 0.035 & 0.034 & 0.064 \\
\hline 189 & Simalungun & 4.112 & 0.021 & -0.090 & -0.067 & 0.426 & -0.206 & 0.529 & 0.009 & 0.035 & 0.033 & 0.061 \\
\hline 190 & Tapanuli Tengah & 4.014 & 0.021 & -0.078 & -0.078 & 0.414 & -0.316 & 0.524 & 0.009 & 0.035 & 0.034 & 0.062 \\
\hline 191 & Humbang Hasundutan & 4.011 & 0.021 & -0.086 & -0.078 & 0.431 & -1.336 & 0.526 & 0.009 & 0.034 & 0.033 & 0.061 \\
\hline 192 & Langkat & 4.081 & 0.021 & -0.082 & -0.059 & 0.363 & -0.171 & 0.528 & 0.009 & 0.033 & 0.032 & 0.059 \\
\hline 193 & Pakpak Bharat & 3.990 & 0.021 & -0.088 & -0.076 & 0.425 & -0.815 & 0.528 & 0.009 & 0.034 & 0.033 & 0.060 \\
\hline 194 & Binjai & 4.099 & 0.021 & -0.086 & -0.061 & 0.384 & -0.267 & 0.528 & 0.009 & 0.034 & 0.033 & 0.060 \\
\hline 195 & Dairi & 4.018 & 0.020 & -0.089 & -0.073 & 0.418 & -1.025 & 0.529 & 0.009 & 0.033 & 0.032 & 0.059 \\
\hline 196 & Karo & 4.045 & 0.020 & -0.088 & -0.069 & 0.407 & -0.971 & 0.529 & 0.009 & 0.033 & 0.032 & 0.059 \\
\hline 197 & Medan & 4.120 & 0.020 & -0.088 & -0.061 & 0.390 & 0.229 & 0.528 & 0.009 & 0.035 & 0.033 & 0.061 \\
\hline 198 & Tebing Tinggi & 4.145 & 0.020 & -0.092 & -0.064 & 0.417 & 0.201 & 0.528 & 0.009 & 0.036 & 0.034 & 0.063 \\
\hline
\end{tabular}




\begin{tabular}{lllllllllllll}
199 & Deli Serdang & 4.112 & 0.020 & -0.090 & -0.064 & 0.401 & 0.119 & 0.529 & 0.009 & 0.034 & 0.033 & 0.061 \\
200 & Serdang Bedagai & 4.141 & 0.020 & -0.092 & -0.064 & 0.414 & 0.620 & 0.528 & 0.009 & 0.036 & 0.034 & 0.063 \\
201 & Aceh Tenggara & 4.019 & 0.019 & -0.081 & -0.064 & 0.357 & -0.596 & 0.528 & 0.009 & 0.032 & 0.032 & 0.058 \\
202 & Aceh Selatan & 3.985 & 0.018 & -0.079 & -0.066 & 0.350 & -0.605 & 0.526 & 0.009 & 0.033 & 0.032 & 0.060 \\
\hline
\end{tabular}

Annex 15 Revenue without and with insurance for districts that are positively pseudo-significant at 5\% significance level

\begin{tabular}{|c|c|c|c|c|c|c|c|c|c|c|c|c|}
\hline No & District & Island & $\begin{array}{l}\text { Premium } \\
\text { (IDR) }\end{array}$ & $\begin{array}{l}\text { PDSI } \\
\text { Trigger }\end{array}$ & $\begin{array}{l}\text { PDSI } \\
\text { Exit }\end{array}$ & $\begin{array}{l}\text { Tick } \\
\text { (IDR) }\end{array}$ & $\begin{array}{l}\text { Revenue } \\
\text { without } \\
\text { Insurance } \\
\text { (IDR) }\end{array}$ & $\begin{array}{l}\text { SD without } \\
\text { Insurance } \\
\text { (IDR) }\end{array}$ & $\begin{array}{l}\text { Revenue } \\
\text { with } \\
\text { Insurance } \\
\text { (IDR) }\end{array}$ & $\begin{array}{l}\text { SD without } \\
\text { Insurance (IDR) }\end{array}$ & $\begin{array}{l}\text { Change } \\
\text { Revenue }\end{array}$ & $\begin{array}{l}\text { Change } \\
\text { Standard } \\
\text { Deviation }\end{array}$ \\
\hline 1 & Sumatera & Aceh Selatan & 21,000 & -2.057 & -6.907 & 412,371 & $15,975,042$ & $1,044,283$ & $15,973,870$ & $1,044,927$ & $-0.16 \%$ & $0.06 \%$ \\
\hline 2 & Sumatera & Aceh Singkil & 21,000 & -2.057 & -6.907 & 412,371 & $13,884,558$ & 532,715 & $13,946,410$ & 429,230 & $-0.15 \%$ & $-19.43 \%$ \\
\hline 3 & Sumatera & Aceh Tamiang & 21,000 & -2.057 & -6.907 & 412,371 & $16,782,905$ & 669,706 & $16,844,757$ & 575,172 & $-0.13 \%$ & $-14.12 \%$ \\
\hline 4 & Sumatera & Aceh Tenggara & 21,000 & -2.057 & -6.907 & 412,371 & $16,549,998$ & $1,009,462$ & $16,611,851$ & 932,553 & $-0.15 \%$ & $-7.62 \%$ \\
\hline 5 & Sumatera & Agam & 21,000 & -2.057 & -6.907 & 412,371 & $19,166,665$ & $1,155,302$ & $19,359,397$ & $1,294,800$ & $-0.13 \%$ & $12.07 \%$ \\
\hline 6 & Sumatera & Asahan & 21,000 & -2.057 & -6.907 & 412,371 & $17,016,666$ & $1,760,234$ & $17,078,518$ & $1,691,660$ & $-0.13 \%$ & $-3.90 \%$ \\
\hline 7 & Sumatera & Bangka Barat & 21,000 & -2.057 & -6.907 & 412,371 & $11,822,636$ & 958,432 & $11,966,661$ & 943,638 & $-0.18 \%$ & $-1.54 \%$ \\
\hline 8 & Sumatera & Bangka Selatan & 21,000 & -2.057 & -6.907 & 412,371 & $9,016,920$ & $1,020,481$ & $9,160,946$ & $1,003,897$ & $-0.24 \%$ & $-1.63 \%$ \\
\hline 9 & Sumatera & Bangka Tengah & 21,000 & -2.057 & -6.907 & 412,371 & $7,887,603$ & 459,963 & $8,031,629$ & 499,787 & $-0.28 \%$ & $8.66 \%$ \\
\hline 10 & Sumatera & Banyu Asin & 21,000 & -2.057 & -6.907 & 412,371 & $15,539,123$ & 868,504 & $15,648,913$ & 898,075 & $-0.14 \%$ & $3.40 \%$ \\
\hline 11 & Sumatera & Belitung & 21,000 & -2.057 & -6.907 & 412,371 & $10,216,665$ & $4,603,828$ & $10,360,691$ & $4,521,924$ & $-0.34 \%$ & $-1.78 \%$ \\
\hline 12 & Sumatera & Belitung Timur & 21,000 & -2.057 & -6.907 & 412,371 & $12,527,316$ & 569,928 & $12,671,341$ & 617,018 & $-0.18 \%$ & $8.26 \%$ \\
\hline 13 & Sumatera & Bengkalis & 21,000 & -2.057 & -6.907 & 412,371 & $12,483,332$ & 704,416 & $12,834,895$ & 661,416 & $-0.18 \%$ & $-6.10 \%$ \\
\hline 14 & Sumatera & Bengkulu & 21,000 & -2.057 & -6.907 & 412,371 & $15,523,936$ & $1,545,988$ & $15,671,837$ & $1,573,193$ & $-0.17 \%$ & $1.76 \%$ \\
\hline 15 & Sumatera & Bengkulu Utara & 21,000 & -2.057 & -6.907 & 412,371 & $13,033,332$ & $1,423,804$ & $13,181,233$ & $1,417,611$ & $-0.19 \%$ & $-0.43 \%$ \\
\hline 16 & Sumatera & Binjai & 21,000 & -2.057 & -6.907 & 412,371 & $17,194,506$ & $1,285,039$ & $17,256,358$ & $1,209,724$ & $-0.14 \%$ & $-5.86 \%$ \\
\hline 17 & Sumatera & Bukit Tinggi & 21,000 & -2.057 & -6.907 & 412,371 & $19,575,997$ & $2,417,954$ & $19,768,728$ & $2,398,831$ & $-0.13 \%$ & $-0.79 \%$ \\
\hline 18 & Sumatera & Bungo & 21,000 & -2.057 & -6.907 & 412,371 & $14,116,666$ & $4,710,159$ & $14,309,397$ & $4,589,324$ & $-0.23 \%$ & $-2.57 \%$ \\
\hline 19 & Sumatera & Dairi & 21,000 & -2.057 & -6.907 & 412,371 & $14,016,666$ & $2,182,700$ & $14,078,518$ & $2,114,854$ & $-0.18 \%$ & $-3.11 \%$ \\
\hline
\end{tabular}




\begin{tabular}{|c|c|c|c|c|c|c|c|c|c|c|c|c|}
\hline 20 & Sumatera & Deli Serdang & 21,000 & -2.057 & -6.907 & 412,371 & $18,516,666$ & $1,478,040$ & $18,578,518$ & $1,422,588$ & $-0.12 \%$ & $-3.75 \%$ \\
\hline 21 & Sumatera & Dharmas Raya & 21,000 & -2.057 & -6.907 & 412,371 & $16,931,906$ & 444,464 & $17,124,637$ & 551,658 & $-0.12 \%$ & $24.12 \%$ \\
\hline 22 & Sumatera & Dumai & 21,000 & -2.057 & -6.907 & 412,371 & $10,440,623$ & 869,281 & $10,792,186$ & 812,292 & $-0.21 \%$ & $-6.56 \%$ \\
\hline 23 & Sumatera & Indragiri Hilir & 21,000 & -2.057 & -6.907 & 412,371 & $17,535,882$ & 505,962 & $17,687,820$ & 646,262 & $-0.12 \%$ & $27.73 \%$ \\
\hline 24 & Sumatera & Indragiri Hulu & 21,000 & -2.057 & -6.907 & 412,371 & $13,933,332$ & 981,891 & $14,047,504$ & 966,330 & $-0.17 \%$ & $-1.58 \%$ \\
\hline 25 & Sumatera & Jambi & 21,000 & -2.057 & -6.907 & 412,371 & $14,059,664$ & $4,510,326$ & $14,173,835$ & $4,458,660$ & $-0.23 \%$ & $-1.15 \%$ \\
\hline 26 & Sumatera & Kampar & 21,000 & -2.057 & -6.907 & 412,371 & $11,366,666$ & $1,307,924$ & $11,718,228$ & $1,104,831$ & $-0.21 \%$ & $-15.53 \%$ \\
\hline 27 & Sumatera & Karo & 21,000 & -2.057 & -6.907 & 412,371 & $14,299,999$ & $2,122,675$ & $14,361,851$ & $2,062,452$ & $-0.17 \%$ & $-2.84 \%$ \\
\hline 28 & Sumatera & Kepahiang & 21,000 & -2.057 & -6.907 & 412,371 & $16,498,359$ & $1,179,338$ & $16,608,149$ & $1,172,966$ & $-0.13 \%$ & $-0.54 \%$ \\
\hline 29 & Sumatera & Kepulauan Mentawai & 21,000 & -2.057 & -6.907 & 412,371 & $14,198,289$ & $1,361,247$ & $14,354,703$ & $1,380,604$ & $0.65 \%$ & $1.42 \%$ \\
\hline 30 & Sumatera & Kerinci & 21,000 & -2.057 & -6.907 & 412,371 & $19,549,998$ & $3,564,468$ & $19,742,729$ & $3,383,357$ & $-0.11 \%$ & $-5.08 \%$ \\
\hline 31 & Sumatera & Kuantan Singingi & 21,000 & -2.057 & -6.907 & 412,371 & $14,475,965$ & $1,497,680$ & $14,668,696$ & $1,547,204$ & $-0.15 \%$ & $3.31 \%$ \\
\hline 32 & Sumatera & Labuhan Batu & 21,000 & -2.057 & -6.907 & 412,371 & $16,049,999$ & $1,213,495$ & $16,206,413$ & $1,246,369$ & $0.58 \%$ & $2.71 \%$ \\
\hline 33 & Sumatera & Lahat & 21,000 & -2.057 & -6.907 & 412,371 & $15,373,761$ & 516,689 & $15,483,551$ & 580,982 & $-0.15 \%$ & $12.44 \%$ \\
\hline 34 & Sumatera & Langkat & 21,000 & -2.057 & -6.907 & 412,371 & $16,999,999$ & $1,808,883$ & $17,061,851$ & $1,744,611$ & $-0.13 \%$ & $-3.55 \%$ \\
\hline 35 & Sumatera & Langsa & 21,000 & -2.057 & -6.907 & 412,371 & $15,662,664$ & 565,035 & $15,724,516$ & 473,475 & $-0.13 \%$ & $-16.20 \%$ \\
\hline 36 & Sumatera & Lebong & 21,000 & -2.057 & -6.907 & 412,371 & $16,837,328$ & 898,142 & $16,985,228$ & 964,526 & $-0.13 \%$ & $7.39 \%$ \\
\hline 37 & Sumatera & Lima Puluh & 21,000 & -2.057 & -6.907 & 412,371 & $18,333,332$ & 606,128 & $18,526,063$ & 773,437 & $-0.11 \%$ & $27.60 \%$ \\
\hline 38 & Sumatera & Lubuk Linggau & 21,000 & -2.057 & -6.907 & 412,371 & $14,581,613$ & 731,662 & $14,691,403$ & 761,301 & $-0.15 \%$ & $4.05 \%$ \\
\hline 39 & Sumatera & Mandailing Natal & 21,000 & -2.057 & -6.907 & 412,371 & $17,689,154$ & 429,903 & $17,845,568$ & 578,743 & $0.50 \%$ & $34.62 \%$ \\
\hline 40 & Sumatera & Medan & 21,000 & -2.057 & -6.907 & 412,371 & $17,736,826$ & 559,880 & $17,798,678$ & 469,351 & $-0.12 \%$ & $-16.17 \%$ \\
\hline 41 & Sumatera & Merangin & 21,000 & -2.057 & -6.907 & 412,371 & $15,223,585$ & $3,345,204$ & $15,371,485$ & $3,385,062$ & $-0.14 \%$ & $1.19 \%$ \\
\hline 42 & Sumatera & Muara Enim & 21,000 & -2.057 & -6.907 & 412,371 & $12,214,032$ & $1,349,892$ & $12,323,822$ & $1,297,702$ & $-0.20 \%$ & $-3.87 \%$ \\
\hline 43 & Sumatera & Muaro Jambi & 21,000 & -2.057 & -6.907 & 412,371 & $15,855,088$ & $3,618,006$ & $15,969,260$ & $3,609,241$ & $-0.14 \%$ & $-0.24 \%$ \\
\hline 44 & Sumatera & Muko & 21,000 & -2.057 & -6.907 & 412,371 & $14,428,977$ & 575,880 & $14,576,877$ & 664,347 & $-0.15 \%$ & $15.36 \%$ \\
\hline 45 & Sumatera & Musi Banyu Asin & 21,000 & -2.057 & -6.907 & 412,371 & $13,799,999$ & $1,725,401$ & $13,914,170$ & $1,679,022$ & $-0.18 \%$ & $-2.69 \%$ \\
\hline 46 & Sumatera & Musi Rawas & 21,000 & -2.057 & -6.907 & 412,371 & $14,348,954$ & $1,529,845$ & $14,458,745$ & $1,481,512$ & $-0.16 \%$ & $-3.16 \%$ \\
\hline 47 & Sumatera & Nias & 21,000 & -2.057 & -6.907 & 412,371 & $13,433,332$ & $1,226,671$ & $13,589,746$ & $1,240,549$ & $0.76 \%$ & $1.13 \%$ \\
\hline 48 & Sumatera & Nias Selatan & 21,000 & -2.057 & -6.907 & 412,371 & $14,982,986$ & 442,979 & $15,139,400$ & 613,187 & $0.60 \%$ & $38.42 \%$ \\
\hline 49 & Sumatera & Ogan Ilir & 21,000 & -2.057 & -6.907 & 412,371 & $15,220,044$ & 507,519 & $15,329,834$ & 560,802 & $-0.14 \%$ & $10.50 \%$ \\
\hline
\end{tabular}




\begin{tabular}{|c|c|c|c|c|c|c|c|c|c|c|c|c|}
\hline 50 & Sumatera & Ogan Komering Ilir & 21,000 & -2.057 & -6.907 & 412,371 & $13,949,999$ & $1,635,384$ & $14,094,024$ & $1,547,582$ & $-0.17 \%$ & $-5.37 \%$ \\
\hline 51 & Sumatera & Padang & 21,000 & -2.057 & -6.907 & 412,371 & $19,275,073$ & $1,570,274$ & $19,467,805$ & $1,648,101$ & $-0.11 \%$ & $4.96 \%$ \\
\hline 52 & Sumatera & Padang Panjang & 21,000 & -2.057 & -6.907 & 412,371 & $18,883,575$ & $1,125,068$ & $19,076,307$ & $1,137,188$ & $-0.11 \%$ & $1.08 \%$ \\
\hline 53 & Sumatera & Padang Pariaman & 21,000 & -2.057 & -6.907 & 412,371 & $17,916,666$ & $1,015,250$ & $18,109,397$ & $1,045,103$ & $-0.11 \%$ & $2.94 \%$ \\
\hline 54 & Sumatera & Padang Sidimpuan & 21,000 & -2.057 & -6.907 & 412,371 & $18,239,402$ & 679,254 & $18,395,816$ & 849,309 & $0.50 \%$ & $25.04 \%$ \\
\hline 55 & Sumatera & Pakpak Bharat & 21,000 & -2.057 & -6.907 & 412,371 & $13,356,875$ & 658,978 & $13,418,727$ & 576,132 & $-0.17 \%$ & $-12.57 \%$ \\
\hline 56 & Sumatera & Palembang & 21,000 & -2.057 & -6.907 & 412,371 & $12,217,111$ & $1,823,522$ & $12,326,901$ & $1,833,627$ & $-0.18 \%$ & $0.55 \%$ \\
\hline 57 & Sumatera & Pariaman & 21,000 & -2.057 & -6.907 & 412,371 & $18,223,615$ & 787,470 & $18,416,346$ & 912,410 & $-0.12 \%$ & $15.87 \%$ \\
\hline 58 & Sumatera & Pasaman & 21,000 & -2.057 & -6.907 & 412,371 & $17,083,332$ & 463,333 & $17,434,895$ & 749,852 & $-0.13 \%$ & $61.84 \%$ \\
\hline 59 & Sumatera & Pasaman Barat & 21,000 & -2.057 & -6.907 & 412,371 & $16,128,985$ & 566,319 & $16,285,398$ & 745,585 & $0.54 \%$ & $31.65 \%$ \\
\hline 60 & Sumatera & Payakumbuh & 21,000 & -2.057 & -6.907 & 412,371 & $18,225,397$ & 974,049 & $18,418,128$ & 999,246 & $-0.12 \%$ & $2.59 \%$ \\
\hline 61 & Sumatera & Pekanbaru & 21,000 & -2.057 & -6.907 & 412,371 & $10,816,084$ & $2,510,589$ & $11,167,646$ & $2,311,947$ & $-0.36 \%$ & $-7.91 \%$ \\
\hline 62 & Sumatera & Pelalawan & 21,000 & -2.057 & -6.907 & 412,371 & $12,791,654$ & $1,071,155$ & $12,877,811$ & $1,072,678$ & $-0.17 \%$ & $0.14 \%$ \\
\hline 63 & Sumatera & Pematang Siantar & 21,000 & -2.057 & -6.907 & 412,371 & $18,585,127$ & $2,265,800$ & $18,646,979$ & $2,221,197$ & $-0.19 \%$ & $-1.97 \%$ \\
\hline 64 & Sumatera & Pesisir Selatan & 21,000 & -2.057 & -6.907 & 412,371 & $18,816,666$ & 918,484 & $19,009,397$ & $1,161,622$ & $-0.11 \%$ & $26.47 \%$ \\
\hline 65 & Sumatera & Prabumulih & 21,000 & -2.057 & -6.907 & 412,371 & $10,758,216$ & 661,432 & $10,868,006$ & 648,237 & $-0.21 \%$ & $-1.99 \%$ \\
\hline 66 & Sumatera & Rejang Lebong & 21,000 & -2.057 & -6.907 & 412,371 & $15,216,666$ & $1,707,343$ & $15,326,456$ & $1,653,995$ & $-0.15 \%$ & $-3.12 \%$ \\
\hline 67 & Sumatera & Rokan Hilir & 21,000 & -2.057 & -6.907 & 412,371 & $13,637,068$ & 761,430 & $13,988,630$ & 814,161 & $-0.17 \%$ & $6.93 \%$ \\
\hline 68 & Sumatera & Rokan Hulu & 21,000 & -2.057 & -6.907 & 412,371 & $9,981,502$ & 726,126 & $10,333,064$ & 736,475 & $-0.22 \%$ & $1.43 \%$ \\
\hline 69 & Sumatera & Samosir & 21,000 & -2.057 & -6.907 & 412,371 & $18,345,984$ & 827,752 & $18,407,836$ & 769,207 & $-0.11 \%$ & $-7.07 \%$ \\
\hline 70 & Sumatera & Sarolangun & 21,000 & -2.057 & -6.907 & 412,371 & $13,366,666$ & $4,961,001$ & $13,480,838$ & $4,873,397$ & $-0.20 \%$ & $-1.77 \%$ \\
\hline 71 & Sumatera & Sawah Lunto & 21,000 & -2.057 & -6.907 & 412,371 & $17,977,788$ & $1,256,214$ & $18,170,519$ & $1,237,778$ & $-0.10 \%$ & $-1.47 \%$ \\
\hline 72 & Sumatera & Seluma & 21,000 & -2.057 & -6.907 & 412,371 & $14,994,634$ & 495,556 & $15,104,424$ & 535,016 & $-0.15 \%$ & $7.96 \%$ \\
\hline 73 & Sumatera & Serdang Bedagai & 21,000 & -2.057 & -6.907 & 412,371 & $19,461,819$ & $1,022,295$ & $19,523,671$ & 982,864 & $-0.11 \%$ & $-3.86 \%$ \\
\hline 74 & Sumatera & Siak & 21,000 & -2.057 & -6.907 & 412,371 & $13,975,691$ & $1,296,498$ & $14,327,254$ & $1,327,556$ & $-0.15 \%$ & $2.40 \%$ \\
\hline 75 & Sumatera & Sijunjung & 21,000 & -2.057 & -6.907 & 412,371 & $15,983,332$ & $1,266,782$ & $16,176,063$ & $1,113,963$ & $-0.13 \%$ & $-12.06 \%$ \\
\hline 76 & Sumatera & Simalungun & 21,000 & -2.057 & -6.907 & 412,371 & $17,533,332$ & $1,839,471$ & $17,595,184$ & $1,787,298$ & $-0.13 \%$ & $-2.84 \%$ \\
\hline 77 & Sumatera & Solok & 21,000 & -2.057 & -6.907 & 412,371 & $18,616,666$ & $1,072,224$ & $18,809,397$ & $1,029,575$ & $-0.11 \%$ & $-3.98 \%$ \\
\hline 78 & Sumatera & Solok Kota & 21,000 & -2.057 & -6.907 & 412,371 & $19,023,007$ & $2,113,726$ & $19,215,738$ & $2,110,521$ & $-0.14 \%$ & $-0.15 \%$ \\
\hline 79 & Sumatera & Solok Selatan & 21,000 & -2.057 & -6.907 & 412,371 & $19,187,356$ & 743,128 & $19,380,088$ & 836,229 & $-0.11 \%$ & $12.53 \%$ \\
\hline
\end{tabular}




\begin{tabular}{|c|c|c|c|c|c|c|c|c|c|c|c|c|}
\hline 80 & Sumatera & Tanah Datar & 21,000 & -2.057 & -6.907 & 412,371 & $20,766,666$ & $1,230,784$ & $20,959,397$ & $1,358,375$ & $-0.11 \%$ & $10.37 \%$ \\
\hline 81 & Sumatera & Tanjung Balai & 21,000 & -2.057 & -6.907 & 412,371 & $16,463,937$ & 851,421 & $16,525,789$ & 812,773 & $-0.13 \%$ & $-4.54 \%$ \\
\hline 82 & Sumatera & Tanjung Jabung Barat & 21,000 & -2.057 & -6.907 & 412,371 & $14,299,999$ & $2,771,595$ & $14,414,171$ & $2,745,132$ & $-0.18 \%$ & $-0.95 \%$ \\
\hline 83 & Sumatera & Tanjung Jabung Timur & 21,000 & -2.057 & -6.907 & 412,371 & $14,283,333$ & $2,298,028$ & $14,397,504$ & $2,259,091$ & $-0.18 \%$ & $-1.69 \%$ \\
\hline 84 & Sumatera & Tapanuli Selatan & 21,000 & -2.057 & -6.907 & 412,371 & $17,733,332$ & $1,030,885$ & $17,889,746$ & $1,119,541$ & $0.55 \%$ & $8.60 \%$ \\
\hline 85 & Sumatera & Tapanuli Tengah & 21,000 & -2.057 & -6.907 & 412,371 & $15,416,666$ & $1,112,859$ & $15,573,079$ & $1,140,789$ & $0.60 \%$ & $2.51 \%$ \\
\hline 86 & Sumatera & Tapanuli Utara & 21,000 & -2.057 & -6.907 & 412,371 & $15,999,999$ & $1,162,069$ & $16,156,413$ & $1,175,984$ & $0.57 \%$ & $1.20 \%$ \\
\hline 87 & Sumatera & Tebing Tinggi & 21,000 & -2.057 & -6.907 & 412,371 & $17,853,947$ & 600,030 & $17,915,799$ & 534,725 & $-0.12 \%$ & $-10.88 \%$ \\
\hline 88 & Sumatera & Tebo & 21,000 & -2.057 & -6.907 & 412,371 & $15,283,325$ & $2,938,534$ & $15,476,056$ & $2,966,734$ & $-0.14 \%$ & $0.96 \%$ \\
\hline 89 & Sumatera & Toba Samosir & 21,000 & -2.057 & -6.907 & 412,371 & $18,269,399$ & $1,592,297$ & $18,425,813$ & $1,726,747$ & $0.47 \%$ & $8.44 \%$ \\
\hline 90 & Sulawesi & Banggai & 24,000 & -2.51 & -7.583 & 394,244 & $14,264,358$ & $2,127,583$ & $14,637,353$ & $2,263,057$ & $-0.20 \%$ & $6.37 \%$ \\
\hline 91 & Sulawesi & Bantaeng & 24,000 & -2.51 & -7.583 & 394,244 & $19,456,369$ & $1,164,339$ & $19,504,318$ & $1,135,820$ & $-0.13 \%$ & $-2.45 \%$ \\
\hline 92 & Sulawesi & Barru & 24,000 & -2.51 & -7.583 & 394,244 & $19,031,385$ & $1,602,672$ & $19,174,405$ & $1,488,288$ & $-0.12 \%$ & $-7.14 \%$ \\
\hline 93 & Sulawesi & Bau & 24,000 & -2.51 & -7.583 & 394,244 & $14,148,132$ & $1,236,788$ & $14,196,081$ & $1,202,309$ & $-0.18 \%$ & $-2.79 \%$ \\
\hline 94 & Sulawesi & Bitung & 24,000 & -2.51 & -7.583 & 394,244 & $15,340,647$ & $2,195,366$ & $15,554,753$ & $2,118,250$ & $-0.24 \%$ & $-3.51 \%$ \\
\hline 95 & Sulawesi & Boalemo & 24,000 & -2.51 & -7.583 & 394,244 & $18,146,242$ & 849,684 & $18,325,934$ & 831,662 & $-0.13 \%$ & $-2.12 \%$ \\
\hline 96 & Sulawesi & Bolaang Mongondow & 24,000 & -2.51 & -7.583 & 394,244 & $17,403,181$ & $2,048,265$ & $17,524,969$ & $1,947,607$ & $-0.17 \%$ & $-4.91 \%$ \\
\hline 97 & Sulawesi & Bombana & 24,000 & -2.51 & -7.583 & 394,244 & $16,024,598$ & 656,037 & $16,072,547$ & 635,785 & $-0.15 \%$ & $-3.09 \%$ \\
\hline 98 & Sulawesi & Bone & 24,000 & -2.51 & -7.583 & 394,244 & $17,813,515$ & $1,064,630$ & $17,861,464$ & $1,060,521$ & $-0.13 \%$ & $-0.39 \%$ \\
\hline 99 & Sulawesi & Bone Bolango & 24,000 & -2.51 & -7.583 & 394,244 & $18,564,463$ & $1,118,271$ & $18,686,251$ & $1,089,108$ & $-0.13 \%$ & $-2.61 \%$ \\
\hline 100 & Sulawesi & Bulukumba & 24,000 & -2.51 & -7.583 & 394,244 & $17,855,238$ & $1,303,082$ & $17,903,187$ & $1,282,406$ & $-0.16 \%$ & $-1.59 \%$ \\
\hline 101 & Sulawesi & Buton & 24,000 & -2.51 & -7.583 & 394,244 & $12,675,154$ & $1,625,738$ & $12,723,103$ & $1,591,724$ & $-0.22 \%$ & $-2.09 \%$ \\
\hline 102 & Sulawesi & Donggala & 24,000 & -2.51 & -7.583 & 394,244 & $16,035,362$ & $2,178,270$ & $16,078,876$ & $2,185,921$ & $-0.19 \%$ & $0.35 \%$ \\
\hline 103 & Sulawesi & Enrekang & 24,000 & -2.51 & -7.583 & 394,244 & $16,890,030$ & 977,144 & $17,033,050$ & $1,026,643$ & $-0.17 \%$ & $5.07 \%$ \\
\hline 104 & Sulawesi & Gorontalo & 24,000 & -2.51 & -7.583 & 394,244 & $18,222,235$ & 782,569 & $18,344,023$ & 703,740 & $-0.13 \%$ & $-10.07 \%$ \\
\hline 105 & Sulawesi & Gorontalo Kota & 24,000 & -2.51 & -7.583 & 394,244 & $19,274,403$ & $1,091,768$ & $19,396,191$ & $1,042,789$ & $-0.12 \%$ & $-4.49 \%$ \\
\hline 106 & Sulawesi & Gowa & 24,000 & -2.51 & -7.583 & 394,244 & $18,836,622$ & 484,996 & $18,979,641$ & 584,309 & $-0.13 \%$ & $20.48 \%$ \\
\hline 107 & Sulawesi & Jeneponto & 24,000 & -2.51 & -7.583 & 394,244 & $17,497,796$ & $2,900,956$ & $17,640,816$ & $2,772,085$ & $-0.18 \%$ & $-4.44 \%$ \\
\hline 108 & Sulawesi & Kendari & 24,000 & -2.51 & -7.583 & 394,244 & $12,901,236$ & $2,567,841$ & $12,949,185$ & $2,554,822$ & $-0.19 \%$ & $-0.51 \%$ \\
\hline 109 & Sulawesi & Kepulauan Selayar & 24,000 & -2.51 & -7.583 & 394,244 & $12,611,479$ & $3,195,565$ & $12,659,428$ & $3,155,312$ & $-0.34 \%$ & $-1.26 \%$ \\
\hline
\end{tabular}




\begin{tabular}{|c|c|c|c|c|c|c|c|c|c|c|c|c|}
\hline 110 & Sulawesi & Kolaka & 24,000 & -2.51 & -7.583 & 394,244 & $15,569,543$ & $1,313,832$ & $15,617,492$ & $1,282,503$ & $-0.17 \%$ & $-2.38 \%$ \\
\hline 111 & Sulawesi & Kolaka Utara & 24,000 & -2.51 & -7.583 & 394,244 & $15,169,765$ & 475,452 & $15,217,715$ & 458,703 & $-0.16 \%$ & $-3.52 \%$ \\
\hline 112 & Sulawesi & Konawe & 24,000 & -2.51 & -7.583 & 394,244 & $16,444,987$ & 447,284 & $16,492,936$ & 429,212 & $-0.15 \%$ & $-4.04 \%$ \\
\hline 113 & Sulawesi & Konawe Selatan & 24,000 & -2.51 & -7.583 & 394,244 & $15,518,853$ & 659,739 & $15,566,802$ & 619,834 & $-0.16 \%$ & $-6.05 \%$ \\
\hline 114 & Sulawesi & Luwu & 24,000 & -2.51 & -7.583 & 394,244 & $18,006,069$ & $1,548,137$ & $18,054,018$ & $1,532,712$ & $-0.18 \%$ & $-1.00 \%$ \\
\hline 115 & Sulawesi & Luwu Timur & 24,000 & -2.51 & -7.583 & 394,244 & $18,384,025$ & 499,981 & $18,431,975$ & 472,450 & $-0.13 \%$ & $-5.51 \%$ \\
\hline 116 & Sulawesi & Luwu Utara & 24,000 & -2.51 & -7.583 & 394,244 & $17,419,092$ & 583,933 & $17,792,087$ & 598,924 & $-0.14 \%$ & $2.57 \%$ \\
\hline 117 & Sulawesi & Majene & 24,000 & -2.51 & -7.583 & 394,244 & $16,189,911$ & $1,325,787$ & $16,332,931$ & $1,292,376$ & $-0.15 \%$ & $-2.52 \%$ \\
\hline 118 & Sulawesi & Makassar & 24,000 & -2.51 & -7.583 & 394,244 & $16,487,068$ & 827,969 & $16,630,087$ & 784,254 & $-0.16 \%$ & $-5.28 \%$ \\
\hline 119 & Sulawesi & Mamasa & 24,000 & -2.51 & -7.583 & 394,244 & $16,444,813$ & 625,883 & $16,587,833$ & 477,555 & $-0.15 \%$ & $-23.70 \%$ \\
\hline 120 & Sulawesi & Mamuju & 24,000 & -2.51 & -7.583 & 394,244 & $18,622,716$ & 580,111 & $18,666,230$ & 587,835 & $-0.13 \%$ & $1.33 \%$ \\
\hline 121 & Sulawesi & Mamuju Utara & 24,000 & -2.51 & -7.583 & 394,244 & $18,171,261$ & 574,474 & $18,214,775$ & 593,448 & $-0.13 \%$ & $3.30 \%$ \\
\hline 122 & Sulawesi & Manado & 24,000 & -2.51 & -7.583 & 394,244 & $11,644,474$ & $1,976,166$ & $11,766,262$ & $2,001,135$ & $-0.17 \%$ & $1.26 \%$ \\
\hline 123 & Sulawesi & Maros & 24,000 & -2.51 & -7.583 & 394,244 & $21,201,451$ & 713,122 & $21,344,470$ & 685,181 & $-0.12 \%$ & $-3.92 \%$ \\
\hline 124 & Sulawesi & Minahasa & 24,000 & -2.51 & -7.583 & 394,244 & $17,420,882$ & $1,673,913$ & $17,542,670$ & $1,594,608$ & $-0.16 \%$ & $-4.74 \%$ \\
\hline 125 & Sulawesi & Minahasa Selatan & 24,000 & -2.51 & -7.583 & 394,244 & $18,204,829$ & 806,928 & $18,326,617$ & 786,211 & $-0.13 \%$ & $-2.57 \%$ \\
\hline 126 & Sulawesi & Minahasa Utara & 24,000 & -2.51 & -7.583 & 394,244 & $15,753,270$ & $1,040,196$ & $15,967,377$ & $1,001,541$ & $-0.15 \%$ & $-3.72 \%$ \\
\hline 127 & Sulawesi & Morowali & 24,000 & -2.51 & -7.583 & 394,244 & $14,842,060$ & 727,979 & $15,215,055$ & 720,703 & $-0.16 \%$ & $-1.00 \%$ \\
\hline 128 & Sulawesi & Muna & 24,000 & -2.51 & -7.583 & 394,244 & $9,914,569$ & $3,089,183$ & $9,962,518$ & $3,051,417$ & $-0.37 \%$ & $-1.22 \%$ \\
\hline 129 & Sulawesi & Palopo & 24,000 & -2.51 & -7.583 & 394,244 & $18,847,962$ & 923,767 & $18,895,912$ & 909,190 & $-0.13 \%$ & $-1.58 \%$ \\
\hline 130 & Sulawesi & Palu & 24,000 & -2.51 & -7.583 & 394,244 & $16,240,927$ & $1,731,866$ & $16,284,441$ & $1,708,103$ & $-0.15 \%$ & $-1.37 \%$ \\
\hline 131 & Sulawesi & Pangkajene \& Kepulauan & 24,000 & -2.51 & -7.583 & 394,244 & $20,096,725$ & $1,118,762$ & $20,239,745$ & $1,118,421$ & $-0.12 \%$ & $-0.03 \%$ \\
\hline 132 & Sulawesi & Pare-pare & 24,000 & -2.51 & -7.583 & 394,244 & $17,325,549$ & $1,349,259$ & $17,468,569$ & $1,243,637$ & $-0.17 \%$ & $-7.83 \%$ \\
\hline 133 & Sulawesi & Parigi Moutong & 24,000 & -2.51 & -7.583 & 394,244 & $19,477,705$ & 794,327 & $19,657,397$ & 679,451 & $-0.12 \%$ & $-14.46 \%$ \\
\hline 134 & Sulawesi & Pinrang & 24,000 & -2.51 & -7.583 & 394,244 & $19,791,177$ & $1,473,276$ & $19,934,196$ & $1,439,267$ & $-0.14 \%$ & $-2.31 \%$ \\
\hline 135 & Sulawesi & Pohuwato & 24,000 & -2.51 & -7.583 & 394,244 & $18,964,103$ & $1,044,433$ & $19,143,795$ & 942,510 & $-0.13 \%$ & $-9.76 \%$ \\
\hline 136 & Sulawesi & Polewali Mandar & 24,000 & -2.51 & -7.583 & 394,244 & $19,402,589$ & 726,142 & $19,545,609$ & 599,403 & $-0.13 \%$ & $-17.45 \%$ \\
\hline 137 & Sulawesi & Poso & 24,000 & -2.51 & -7.583 & 394,244 & $13,650,446$ & $2,039,591$ & $14,023,441$ & $2,260,543$ & $-0.22 \%$ & $10.83 \%$ \\
\hline 138 & Sulawesi & Sidenreng Rappang & 24,000 & -2.51 & -7.583 & 394,244 & $20,281,165$ & 830,564 & $20,329,114$ & 833,914 & $-0.11 \%$ & $0.40 \%$ \\
\hline 139 & Sulawesi & Sinjai & 24,000 & -2.51 & -7.583 & 394,244 & $17,036,349$ & $1,964,801$ & $17,084,299$ & $1,940,939$ & $-0.18 \%$ & $-1.21 \%$ \\
\hline
\end{tabular}




\begin{tabular}{|c|c|c|c|c|c|c|c|c|c|c|c|c|}
\hline 140 & Sulawesi & Soppeng & 24,000 & -2.51 & -7.583 & 394,244 & $21,129,111$ & $2,458,493$ & $21,272,130$ & $2,272,883$ & $-0.14 \%$ & $-7.55 \%$ \\
\hline 141 & Sulawesi & Takalar & 24,000 & -2.51 & -7.583 & 394,244 & $18,277,242$ & 849,684 & $18,420,262$ & 873,761 & $-0.12 \%$ & $2.83 \%$ \\
\hline 142 & Sulawesi & Tana Toraja & 24,000 & -2.51 & -7.583 & 394,244 & $16,452,478$ & $1,142,655$ & $16,595,497$ & $1,090,847$ & $-0.17 \%$ & $-4.53 \%$ \\
\hline 143 & Sulawesi & Tojo Una-una & 24,000 & -2.51 & -7.583 & 394,244 & $13,696,161$ & 669,350 & $14,069,157$ & 671,672 & $-0.18 \%$ & $0.35 \%$ \\
\hline 144 & Sulawesi & Tomohon & 24,000 & -2.51 & -7.583 & 394,244 & $18,306,001$ & $1,052,451$ & $18,427,789$ & $1,049,552$ & $-0.13 \%$ & $-0.28 \%$ \\
\hline 145 & Sulawesi & Wajo & 24,000 & -2.51 & -7.583 & 394,244 & $17,483,248$ & $1,099,882$ & $17,531,197$ & $1,086,618$ & $-0.15 \%$ & $-1.21 \%$ \\
\hline 146 & Sulawesi & Wakatobi & 24,000 & -2.51 & -7.583 & 394,244 & $13,541,773$ & 509,688 & $13,589,723$ & 480,299 & $-0.19 \%$ & $-5.77 \%$ \\
\hline 147 & Papua & Asmat & 36,000 & -1.675 & -5.808 & 483,910 & $13,856,663$ & 749,909 & $14,135,607$ & 655,924 & $-0.25 \%$ & $-12.53 \%$ \\
\hline 148 & Papua & Boven Digoel & 36,000 & -1.675 & -5.808 & 483,910 & $9,687,622$ & $1,532,851$ & $10,025,140$ & $1,534,048$ & $-0.38 \%$ & $0.08 \%$ \\
\hline 149 & Papua & Jayapura & 36,000 & -1.675 & -5.808 & 483,910 & $12,256,242$ & $2,110,004$ & $12,260,366$ & $2,105,806$ & $-0.41 \%$ & $-0.20 \%$ \\
\hline 150 & Papua & Jayapura Kota & 36,000 & -1.675 & -5.808 & 483,910 & $13,241,554$ & $1,058,780$ & $13,245,678$ & $1,037,998$ & $-0.29 \%$ & $-1.96 \%$ \\
\hline 151 & Papua & Jayawijaya & 36,000 & -1.675 & -5.808 & 483,910 & $11,603,483$ & $2,293,249$ & $11,607,607$ & $2,292,503$ & $-0.49 \%$ & $-0.03 \%$ \\
\hline 152 & Papua & Keerom & 36,000 & -1.675 & -5.808 & 483,910 & $12,036,266$ & 938,646 & $12,070,130$ & 903,418 & $-0.32 \%$ & $-3.75 \%$ \\
\hline 153 & Papua & Mimika & 36,000 & -1.675 & -5.808 & 483,910 & $11,310,570$ & $2,116,356$ & $11,579,383$ & $1,966,135$ & $-0.48 \%$ & $-7.10 \%$ \\
\hline 154 & Papua & Nabire & 36,000 & -1.675 & -5.808 & 483,910 & $14,232,895$ & 700,882 & $14,501,708$ & 753,213 & $-0.25 \%$ & $7.47 \%$ \\
\hline 155 & Papua & Paniai & 36,000 & -1.675 & -5.808 & 483,910 & $9,965,989$ & $1,516,537$ & $10,234,802$ & $1,383,853$ & $-0.48 \%$ & $-8.75 \%$ \\
\hline 156 & Papua & Peg. Bintang & 36,000 & -1.675 & -5.808 & 483,910 & $11,230,588$ & 574,247 & $11,264,452$ & 589,073 & $-0.35 \%$ & $2.58 \%$ \\
\hline 157 & Papua & Puncak Jaya & 36,000 & -1.675 & -5.808 & 483,910 & $12,775,783$ & 667,706 & $12,779,907$ & 663,024 & $-0.30 \%$ & $-0.70 \%$ \\
\hline 158 & Papua & Sarmi & 36,000 & -1.675 & -5.808 & 483,910 & $11,852,975$ & 897,959 & $11,857,099$ & 893,196 & $-0.33 \%$ & $-0.53 \%$ \\
\hline 159 & Papua & Tolikara & 36,000 & -1.675 & -5.808 & 483,910 & $12,909,712$ & 639,083 & $12,913,836$ & 650,516 & $-0.29 \%$ & $1.79 \%$ \\
\hline 160 & Papua & Waropen & 36,000 & -1.675 & -5.808 & 483,910 & $9,849,958$ & $1,359,504$ & $10,118,771$ & $1,385,524$ & $-0.38 \%$ & $1.91 \%$ \\
\hline 161 & Papua & Yahukimo & 36,000 & -1.675 & -5.808 & 483,910 & $12,826,809$ & 647,154 & $12,830,933$ & 621,055 & $-0.29 \%$ & $-4.03 \%$ \\
\hline 162 & Kalimantan & Balangan & 107,000 & -1.092 & -6.265 & 386,623 & $14,525,719$ & 495,646 & $14,782,386$ & 627,629 & $-0.72 \%$ & $26.63 \%$ \\
\hline 163 & Kalimantan & Balikpapan & 107,000 & -1.092 & -6.265 & 386,623 & $11,613,153$ & $1,875,191$ & $11,869,821$ & $1,918,924$ & $-1.21 \%$ & $2.33 \%$ \\
\hline 164 & Kalimantan & Banjar & 107,000 & -1.092 & -6.265 & 386,623 & $12,478,384$ & $1,860,516$ & $12,660,643$ & $1,866,097$ & $-1.15 \%$ & $0.30 \%$ \\
\hline 165 & Kalimantan & Banjarbaru & 107,000 & -1.092 & -6.265 & 386,623 & $13,118,474$ & 479,085 & $13,177,673$ & 512,675 & $-0.84 \%$ & $7.01 \%$ \\
\hline 166 & Kalimantan & Banjarmasin & 107,000 & -1.092 & -6.265 & 386,623 & $12,976,796$ & 534,561 & $13,035,996$ & 559,535 & $-0.84 \%$ & $4.67 \%$ \\
\hline 167 & Kalimantan & Barito Kuala & 107,000 & -1.092 & -6.265 & 386,623 & $12,344,977$ & $1,923,840$ & $12,404,176$ & $1,944,078$ & $-1.21 \%$ & $1.05 \%$ \\
\hline 168 & Kalimantan & Barito Selatan & 107,000 & -1.092 & -6.265 & 386,623 & $9,118,881$ & $1,489,605$ & $9,172,281$ & $1,462,122$ & $-1.47 \%$ & $-1.84 \%$ \\
\hline 169 & Kalimantan & Barito Timur & 107,000 & -1.092 & -6.265 & 386,623 & $11,047,153$ & 682,586 & $11,303,820$ & 743,939 & $-0.98 \%$ & $8.99 \%$ \\
\hline
\end{tabular}




\begin{tabular}{|c|c|c|c|c|c|c|c|c|c|c|c|c|}
\hline 170 & Kalimantan & Barito Utara & 107,000 & -1.092 & -6.265 & 386,623 & $7,565,495$ & $1,434,672$ & $7,822,162$ & $1,349,714$ & $-1.60 \%$ & $-5.92 \%$ \\
\hline 171 & Kalimantan & Gunung Mas & 107,000 & -1.092 & -6.265 & 386,623 & $7,670,313$ & 628,377 & $7,723,713$ & 676,722 & $-1.44 \%$ & $7.69 \%$ \\
\hline 172 & Kalimantan & Hulu Sungai Selatan & 107,000 & -1.092 & -6.265 & 386,623 & $12,234,485$ & $3,539,719$ & $12,543,113$ & $3,372,991$ & $-1.52 \%$ & $-4.71 \%$ \\
\hline 173 & Kalimantan & Hulu Sungai Tengah & 107,000 & -1.092 & -6.265 & 386,623 & $15,561,308$ & $2,306,878$ & $15,743,567$ & $2,389,584$ & $-0.75 \%$ & $3.59 \%$ \\
\hline 174 & Kalimantan & Hulu Sungai Utara & 107,000 & -1.092 & -6.265 & 386,623 & $15,728,872$ & $2,059,153$ & $15,911,131$ & $2,174,460$ & $-0.70 \%$ & $5.60 \%$ \\
\hline 175 & Kalimantan & Humbang Hasundutan & 107,000 & -1.092 & -6.265 & 386,623 & $15,513,800$ & $4,557,828$ & $15,790,209$ & $4,450,463$ & $3.52 \%$ & $-2.36 \%$ \\
\hline 176 & Kalimantan & Kapuas & 107,000 & -1.092 & -6.265 & 386,623 & $10,404,955$ & $1,527,395$ & $10,458,355$ & $1,488,318$ & $-1.29 \%$ & $-2.56 \%$ \\
\hline 177 & Kalimantan & Kapuas Hulu & 107,000 & -1.092 & -6.265 & 386,623 & $8,756,288$ & $1,068,516$ & $9,002,540$ & $1,078,285$ & $-1.63 \%$ & $0.91 \%$ \\
\hline 178 & Kalimantan & Katingan & 107,000 & -1.092 & -6.265 & 386,623 & $12,196,113$ & $2,640,766$ & $12,249,513$ & $2,612,641$ & $-0.88 \%$ & $-1.07 \%$ \\
\hline 179 & Kalimantan & Ketapang & 107,000 & -1.092 & -6.265 & 386,623 & $10,847,259$ & $1,109,216$ & $11,005,264$ & $1,020,521$ & $-1.22 \%$ & $-8.00 \%$ \\
\hline 180 & Kalimantan & Kota Baru & 107,000 & -1.092 & -6.265 & 386,623 & $12,684,016$ & $1,649,746$ & $12,866,274$ & $1,711,832$ & $-0.82 \%$ & $3.76 \%$ \\
\hline 181 & Kalimantan & Kota Waringin Barat & 107,000 & -1.092 & -6.265 & 386,623 & $8,646,050$ & $1,407,538$ & $8,625,621$ & $1,406,381$ & $-1.65 \%$ & $-0.08 \%$ \\
\hline 182 & Kalimantan & Kota Waringin Timur & 107,000 & -1.092 & -6.265 & 386,623 & $9,561,776$ & 820,016 & $9,615,176$ & 830,877 & $-1.20 \%$ & $1.32 \%$ \\
\hline 183 & Kalimantan & Kutai Barat & 107,000 & -1.092 & -6.265 & 386,623 & $10,880,447$ & 839,122 & $11,137,114$ & 877,729 & $-0.92 \%$ & $4.60 \%$ \\
\hline 184 & Kalimantan & Lamandau & 107,000 & -1.092 & -6.265 & 386,623 & $7,812,116$ & 669,463 & $7,970,122$ & 685,386 & $-1.47 \%$ & $2.38 \%$ \\
\hline 185 & Kalimantan & Melawi & 107,000 & -1.092 & -6.265 & 386,623 & $8,475,414$ & 503,873 & $8,633,420$ & 508,876 & $-1.40 \%$ & $0.99 \%$ \\
\hline 186 & Kalimantan & Murung Raya & 107,000 & -1.092 & -6.265 & 386,623 & $7,840,018$ & 517,565 & $7,893,418$ & 564,952 & $-1.42 \%$ & $9.16 \%$ \\
\hline 187 & Kalimantan & Palangkaraya & 107,000 & -1.092 & -6.265 & 386,623 & $6,879,779$ & $1,153,346$ & $6,933,179$ & $1,173,032$ & $-2.63 \%$ & $1.71 \%$ \\
\hline 188 & Kalimantan & Paser & 107,000 & -1.092 & -6.265 & 386,623 & $11,926,276$ & $1,867,563$ & $12,182,943$ & $1,795,895$ & $-1.29 \%$ & $-3.84 \%$ \\
\hline 189 & Kalimantan & Penajam Paser Utara & 107,000 & -1.092 & -6.265 & 386,623 & $18,644,831$ & $1,011,702$ & $18,901,498$ & $1,059,522$ & $-0.57 \%$ & $4.73 \%$ \\
\hline 190 & Kalimantan & Pulang Pisau & 107,000 & -1.092 & -6.265 & 386,623 & $11,375,288$ & 896,419 & $11,428,688$ & 904,178 & $-0.98 \%$ & $0.87 \%$ \\
\hline 191 & Kalimantan & Seruyan & 107,000 & -1.092 & -6.265 & 386,623 & $8,393,266$ & 506,419 & $8,551,272$ & 548,645 & $-1.35 \%$ & $8.34 \%$ \\
\hline 192 & Kalimantan & Sintang & 107,000 & -1.092 & -6.265 & 386,623 & $8,711,842$ & $1,645,188$ & $8,845,249$ & $1,628,752$ & $-1.93 \%$ & $-1.00 \%$ \\
\hline 193 & Kalimantan & Tabalong & 107,000 & -1.092 & -6.265 & 386,623 & $14,353,537$ & $2,390,117$ & $14,610,204$ & $2,317,033$ & $-1.01 \%$ & $-3.06 \%$ \\
\hline 194 & Kalimantan & Tanah Bumbu & 107,000 & -1.092 & -6.265 & 386,623 & $15,223,862$ & $1,107,378$ & $15,406,120$ & $1,185,327$ & $-0.74 \%$ & $7.04 \%$ \\
\hline 195 & Kalimantan & Tanah Laut & 107,000 & -1.092 & -6.265 & 386,623 & $12,461,802$ & $1,481,453$ & $12,521,001$ & $1,486,668$ & $-0.99 \%$ & $0.35 \%$ \\
\hline 196 & Kalimantan & Tapin & 107,000 & -1.092 & -6.265 & 386,623 & $14,450,365$ & $1,822,706$ & $14,632,623$ & $1,876,816$ & $-0.85 \%$ & $2.97 \%$ \\
\hline 197 & Java & Batang Hari & 187,000 & -0.56 & -2.795 & 894,855 & $22,132,113$ & 653,507 & $22,397,200$ & 934,646 & $-0.85 \%$ & $43.02 \%$ \\
\hline 198 & Java & Sumenep & 187,000 & -0.56 & -2.795 & 894,855 & $18,999,999$ & $2,550,463$ & $19,265,087$ & $2,580,680$ & $-1.15 \%$ & $1.18 \%$ \\
\hline 199 & Balinusa & Dompu & 51,000 & 0.0255 & -2.263 & 873,935 & $15,951,989$ & $2,108,151$ & $16,420,853$ & $2,127,512$ & $4.93 \%$ & $0.92 \%$ \\
\hline
\end{tabular}




\begin{tabular}{|c|c|c|c|c|c|c|c|c|c|c|c|c|}
\hline 200 & Balinusa & Kupang & 51,000 & 0.0255 & -2.263 & 873,935 & $11,928,596$ & 839,982 & $12,124,246$ & 713,874 & $8.56 \%$ & $-15.01 \%$ \\
\hline 201 & Balinusa & Timor Tengah Selatan & 51,000 & 0.0255 & -2.263 & 873,935 & $11,484,020$ & $1,667,470$ & $11,679,670$ & $1,581,056$ & $10.87 \%$ & $-5.18 \%$ \\
\hline 202 & Balinusa & Timor Tengah Utara & 51,000 & 0.0255 & -2.263 & 873,935 & $10,754,316$ & $1,221,259$ & $10,949,966$ & $1,113,083$ & $11.19 \%$ & $-8.86 \%$ \\
\hline
\end{tabular}

Annex 16 Summary report on regression estimates using different bandwidth and GWR models

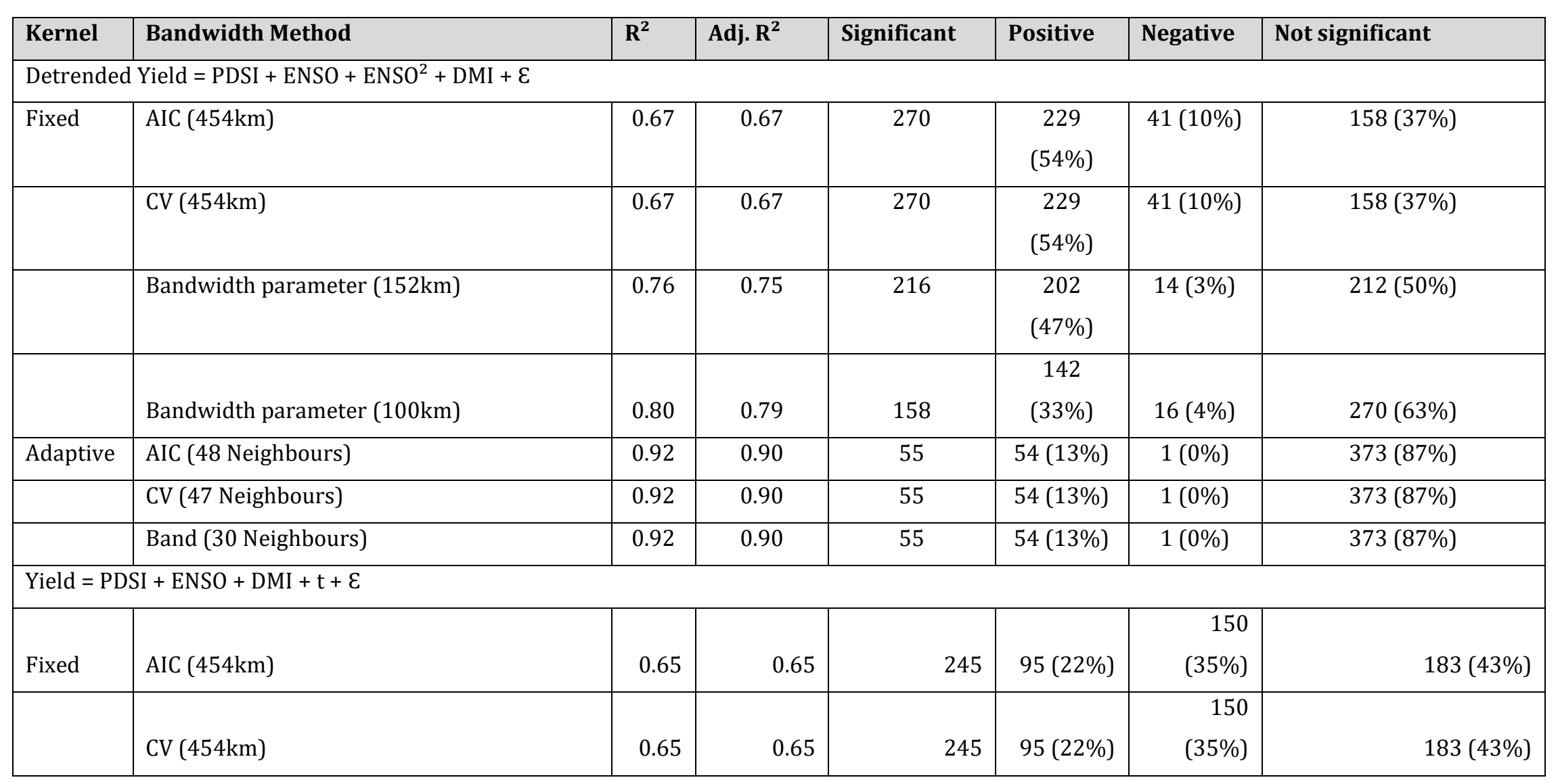




\begin{tabular}{|c|c|c|c|c|c|c|c|}
\hline & Bandwidth para $(152 \mathrm{~km})$ & 0.75 & 0.74 & 162 & $52(12 \%)$ & $\begin{array}{r}110 \\
(26 \%)\end{array}$ & $266(62 \%)$ \\
\hline & Bandwidth para $(100 \mathrm{~km})$ & 0.79 & 0.77 & 127 & $27(6 \%)$ & $\begin{array}{r}100 \\
(23 \%)\end{array}$ & $301(70 \%)$ \\
\hline \multirow[t]{3}{*}{ Adaptive } & AIC (48 Neighbours) & 0.91 & 0.89 & 30 & $8(2 \%)$ & $22(5 \%)$ & $398(93 \%)$ \\
\hline & CV (47 Neighbours) & 0.91 & 0.89 & 30 & $8(2 \%)$ & $22(5 \%)$ & $398(93 \%)$ \\
\hline & Band (30 Neighbours) & 0.92 & 0.90 & 30 & $8(2 \%)$ & $22(5 \%)$ & $398(93 \%)$ \\
\hline
\end{tabular}


Annex 17 Spatial Distribution of t-statistic Results
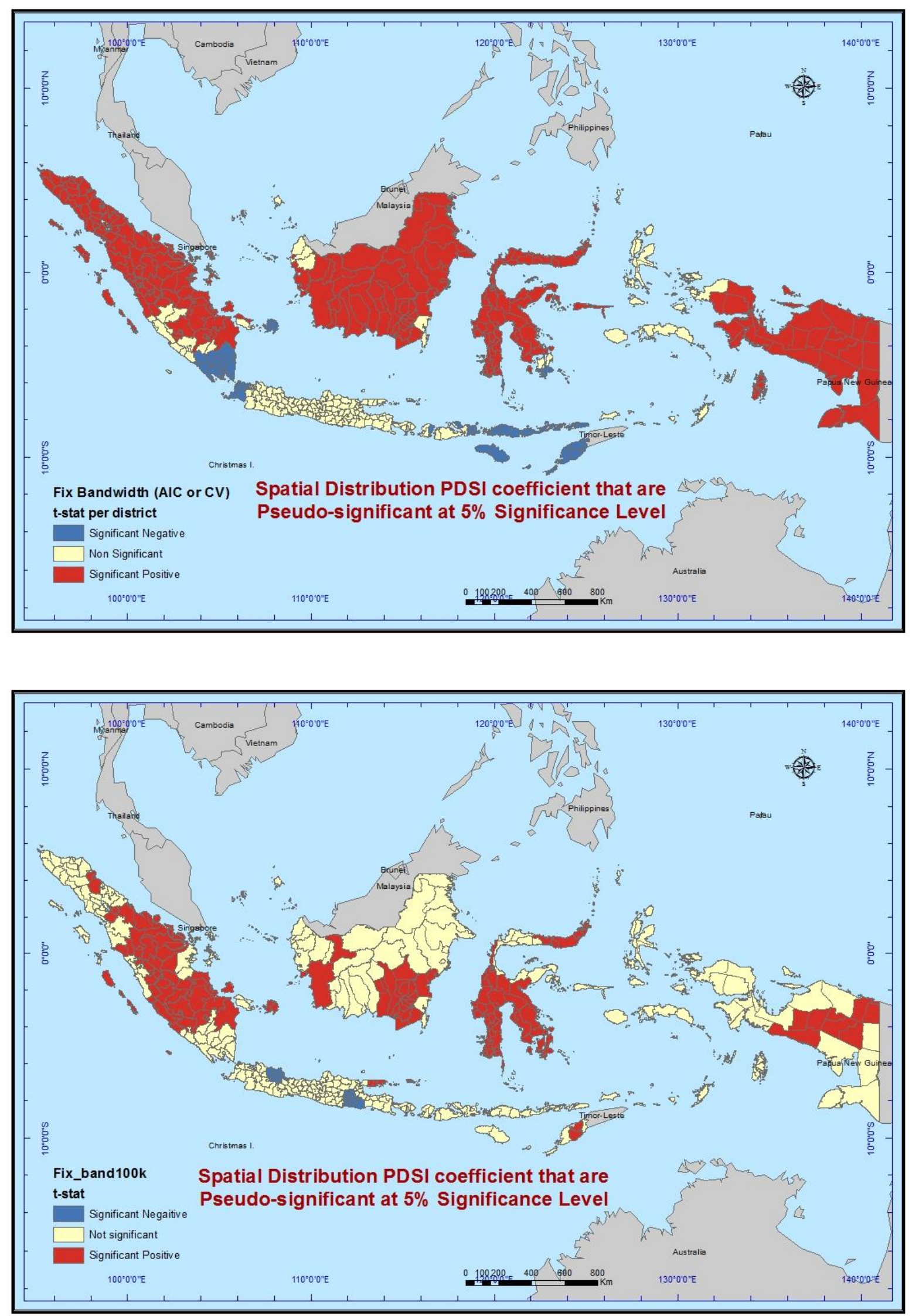

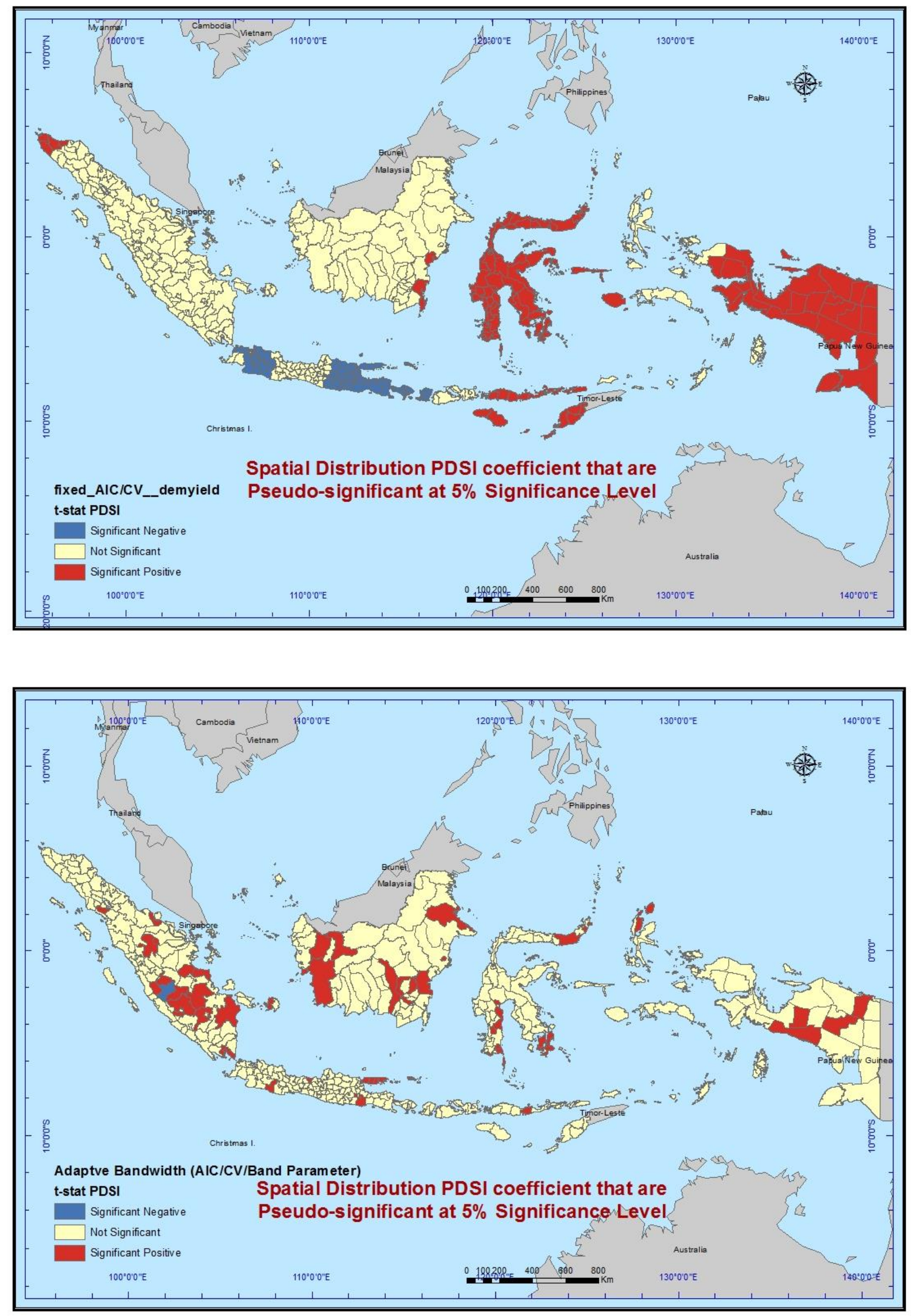


\title{
Chapter THREE
}

\section{DROUGHT CONSEQUENCES ON HOUSEHOLDS: EXPLORING EVIDENCE FROM LONGITUDINAL DATA}

\begin{abstract}
:
This paper examines the short-term impacts of recurring droughts on households using a panel survey. Our identification strategy exploits variations across space and time of historical monthly weather data at the sub-district level and changes in household incomes and expenditures. We use a monthly gridded dataset $\left(0.5^{\circ} \times 0.5^{\circ}\right)$ of the Standardized Precipitation Evapotranspiration Index (SPEI) to construct a measure of drought events from 1990 to 2014. The impacts of these annual exogenous weather shocks on various household economic indicators are then investigated. The household data are gathered from five waves of the Indonesian Family Life Survey (IFLS) - a longitudinal household survey. The results suggest robust evidence of the effects of drought on household's well-being. The scale and significance of the impact depend on several characteristics, especially drought's timing. The findings indicate that a drought occurred last year is associated the most with drops in incomes and expenditures. Generally, impacts on household incomes (between 7\% and 44\%) are larger than impacts on household consumption expenditure (between 1\% and 9\% percentage change). We also find that the drought effects evidently vary between one household group and another, where selling assets is apparently the preferred coping mechanism to respond to adverse drought shock for rich households.
\end{abstract}




\subsection{Introduction}

The adverse impacts of extreme weather events on people and environment may be obvious, as empirical evidence suggests (Devereux, 2007; Cavallo et al., 2013; Dell et al., 2014; Felbermayr \& Gröschl, 2014). In the developing world where infrastructure and social safety nets are not well established, and dependency on the agriculture sector is high, weather-related disasters such as floods, droughts, storms, typhoons, and hurricanes can potentially cause significant losses and long-lasting effects (Noy \& Vu, 2010; Strobl, 2012; Lohmann \& Lechtenfeld, 2015; Karim, 2018; Yonson, 2018). Bastos et al. (2013) show the negative effects of long-term drought on regional labour market outcomes. They also find that a delayed rainy season can reduce consumption of rural households, especially the lowest income ones that mostly depend on agricultural activities (Korkeala et al., 2009; Skoufias et al., 2012). ${ }^{18}$ Lower rainfall than normal at early life or birth has disadvantageous impacts on individual's socio-economic status later on in adulthood (Maccini \& Yang, 2009), and negatively impacts health outcomes (Skoufias \& Vinha, 2012; Lohmann \& Lechtenfeld, 2015; Groppo \& Kraehnert, 2016).

Outside of Indonesia, weather extremes were also found to lead to social unrest due to tougher competition on already depleting livelihood resources, for example in the case of poor countries (Miguel et al., 2004; Hodler \& Raschky, 2014; Maystadt \& Ecker, 2014; Caruso et al., 2016). In the United States, variations of temperature are found to be positively associated with crimes (Ranson, 2014). On the positive side, a study found that rainfall deficits induce more investment in education as the family chooses to send children for schooling as working for agricultural production is not possible nor efficient during a drought period (Shah \& Steinberg, 2017).

Existing literature has observed evidence of weather effects on the economy, but more needs to be done to identify the exact timing of the shocks that potentially impact the local economy. While findings in the current literature suggest a weather-economy nexus, there are a number of methodological flaws that potentially cast doubt on identification of the impact of weather shocks. This occurs, especially, when the study focuses on contemporaneous (short-term) effects and uses only cross-sectional data. Here, we analyse a panel that is used for an empirical investigation of the relationship between the

\footnotetext{
${ }^{18}$ Although Skoufias et al. (2012) found little evidence that weather shocks have generally caused welfare losses to rural households but only to rice farm households located in areas exposed to low rainfall.
} 
timing of drought events and the changes in household outcomes (income and expenditure) over several decades.

In this chapter, we consider the importance of linking historical variation of local climate data and the fluctuation of household outcomes surveyed over several decades to investigate the impact of weather extremes. Our focused weather shock in this study is drought. Palmer (1965) gives his principal definition of drought as "a prolonged and abnormal moisture deficiency". The measurement of dryness is based on recent precipitation and temperature. Here, following Palmer's work, we define drought as "a chronic environmental condition resulting from negative water balance at a particular location within an impactful period“. We determine periodic drought events using the Standardized Precipitation Evapotranspiration Index (SPEI). This drought measure is constructed at the sub-district level based on SPEI global gridded dataset at $0.5 \times 0.5^{\circ}$ spatial resolution (or about $3,099 \mathrm{~km}^{2}$ per grid) and a 12 -monthly time resolution ${ }^{19}$. To this end, we construct drought events into 3 lagged periods (one, two and three years prior to household surveys) and then link these with variations in household economic outcomes, which are observable in the survey.

With this data, we aim to determine whether the variations of weather shocks are associated with the variations of household incomes and welfare. The household income variables we examine include total income, farm income, non-farm income and sales of assets (farm, non-farm, and other assets) in the past 12 months. Lastly, for the household welfare variables, we include per capita monthly expenses for food, non-food, education and for the total consumption.

Indonesia being an archipelagic nation is an interesting case because it has a complex variation of local climates (about 38\% of the labour force in Indonesia works in the agricultural sector). This study uses information from 5 (five) waves of the Indonesian Family Life Survey (IFLS 1993, 1997, 2000, 2007 and 2015). The availability of socioeconomic data at the household level in IFLS is exceptionally instrumental for the empirical analysis conducted in this study. Data in IFLS is known to be high-quality longitudinal household data that have unusually low attrition rates (Mani, 2012; Thomas

\footnotetext{
${ }^{19}$ The global gridded dataset SPEI is a multi-scale (space and time) drought index as elaborated in SPEI master papers (Vicente-Serrano, Beguera, \& Lpez-Moreno, 2010; Vicente-Serrano, Beguera, Lpez-Moreno, et al., 2010). Complete dataset is available online at: http://hdl.handle.net/10261/153475.
} 
et al., 2012; Gignoux \& Menéndez, 2016). This enables our empirical analysis to examine the varying effects of weather shocks on the household economy over the survey periods. This article focuses on drought shock as existing literature suggests it has been a major natural hazard that cause huge losses in low-middle income countries like Indonesia where agricultural production is the main source of income (D'Arrigo \& Wilson, 2008; Lassa, 2012; Solh \& van Ginkel, 2014; FAO, 2015; Surmaini et al., 2015; Baudoin et al., 2017).

Our empirical results generally reveal negative associations between drought and household incomes, except for income from selling assets where we find a positive correlation with droughts. This probably explains why we do not find evidence of the same magnitude of drought-related impact on household expenditure as we do on household income because there is consumption smoothing through selling assets during the shocks. The magnitude and significance of the drought effect are also dependent on the timing of the drought. The potentially negative short-term effects of drought are observable over all 5 waves and we find drought shocks hit the most households that receive income from own business (farm or non-farm).

Section 2 describes the chosen study area and existing works about weather effects on economic outcomes. We describe the data collection and report the descriptive statistics in Section 3. More importantly, in this section, we discuss the conceptual issues and chosen empirical strategy of this paper. In Section 4, we present the main empirical results. Where applicable, we relate findings of the current article with the earlier literature. Finally, Section 5 concludes the paper.

\subsection{Macroeconomic and weather conditions in Indonesia}

Indonesia is a typical developing country where primary sectors like farming and fishing are important livelihoods of a big fraction of the population, especially in rural area. According to the World Bank, more than 38\% of total labour force in Indonesia is involved in the agricultural-related production in which it has a high dependency on nature conditions, such as weather. Indonesia's geographic position in the tropics and between 2 large oceans, Pacific and Indian, provide the archipelagic nation with relatively stable temperature conditions year-round but with high spatial diversity of rainfall across regions. 
Figure 7 presents Indonesia's macroeconomic figures, which in general show stable growth. Total GDP has steadily increased (except during economic crisis 1997 - 1999) from US\$ 88.64 Billion (1970) to US\$1.03 Trillion (2016) with 5-6\% growth rate in the last 15 years. The poverty rate 20 declined sharply from $71.4 \%$ (1984) to $6.5 \%$ (2016) and life expectancy increased from 63 years (1990) to 68 years (2016). The steady increase of GDP agriculture indicates the growing size of the agricultural economy, although its relative share has been decreasing as other sectors like manufacturing and services have grown more rapidly in the last decades. Total cultivated land for crops and animal husbandry is about 57 million ha. Smallholders with farmland less than 1 ha are the majority of the food crop cultivation and livestock production. Most of the agricultural households are concentrated in Java (including Madura), Bali - West Nusa Tenggara and Sumatera islands, being the most fertile areas in Indonesia.

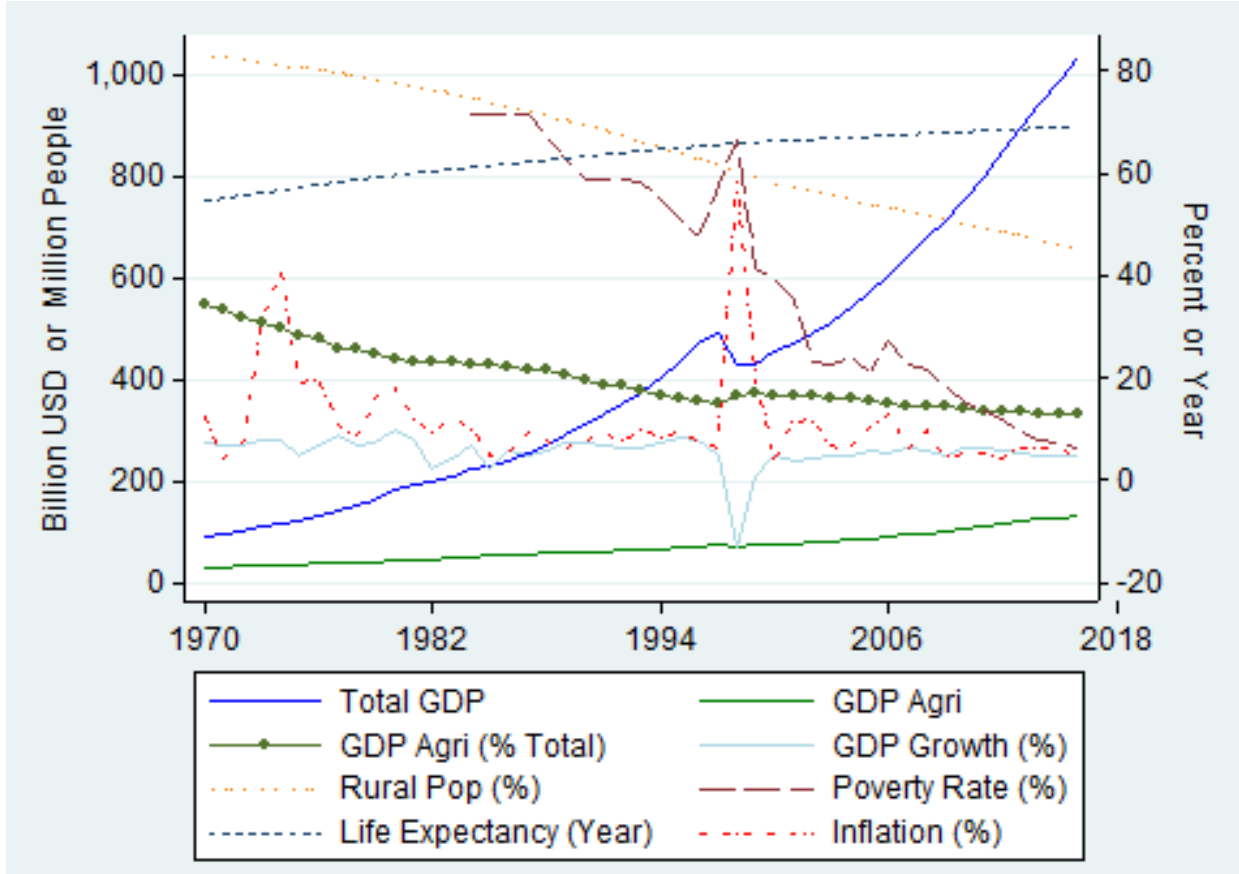

Source: Author's calculation from the World Development Indicator, accessed in May 2018

Figure 7 Macroeconomic Statistics Indonesia 1970 - 2016

Indonesia has a diverse climate, especially with respect to precipitation (see Annex 1, a map of agro-climatic conditions that illustrates regional differences in rainfall). The temperature is constant between $21-25^{\circ} \mathrm{C}$ in the mountain areas and $28-30^{\circ} \mathrm{C}$ in the

\footnotetext{
${ }^{20}$ Poverty headcount ratio at $\$ 1.90$ a day (\% of population) using PPP 2011 . It is defined as the percentage of the population living on less than $\$ 1.90$ a day at 2011 international prices.
} 
coastal areas and not much different between seasons, but with high humidity throughout the year (between 70-90\%). There are only two seasons: the dry and rainy seasons. There are significant differences between coastal areas that have less rain and mountainous areas that have much more rain, up to $6000 \mathrm{~mm}$ per year. At the sub-district level, there are high spatial differences across islands regarding seasonal rainfall. For example, the amount of rainfall in the dry and wet season is not much different in Kalimantan and Sumatera regions, while in Nusa Tenggara there are more distinct variations of rainfall between seasons.

Indonesia is prone to weather-related extreme events. The most extreme example is probably the massive drought in 1997, which was thought to be caused by a strong El Niño, failing hundreds of thousands of hectares of crop harvest (in most areas of Sulawesi, and some parts of Kalimantan and Papua), triggering millions of hectares of forest fires and affecting 1.065 million people. While in the global scene there has been a decrease of fatal casualties from climatic disasters as emergency preparedness and response improved (supported by technological improvements such as satellite monitoring and forecasts), economic losses seem to show an increasing trend. These global phenomena seem to be true also for Indonesia when we observe data from EM-DAT21 (see the graph of Annex 3).

A recent study from FAO (2015) found that the agricultural sector absorbs $84 \%$ of drought's total economic impact, signalling it as the most damaging disaster for developing countries. Many cultivated areas in developing countries are non-irrigated; therefore, production is very sensitive to drought. In Indonesia, for example, irrigation networks only cover $17 \%$ of total cultivated areas (these are mostly in the form of sawah, a wetland for rice cultivation) ${ }^{22}$. Using Indonesia official statistics, Lassa (2012) confirms that drought has the highest impact on crop losses compared to other natural catastrophes, where crop damage probability reaches the rate of 1,182 ha per drought event. This indicates a high degree of susceptibility to drought risks for a majority of households, especially to those whose crop production is their primary livelihood.

\footnotetext{
21 EM-DAT: The Emergency Events Database - Université catholique de Louvain (UCL) - CRED, www.emdat.be, Brussels, Belgium.

${ }^{22}$ A detailed figure of irrigation networks in main islands of Indonesia is presented in Annex 5 (Irrigated Areas in Main Islands of Indonesia in 2016). Online database is available at Indonesia Ministry of Agriculture: http://prasarana.pertanian.go.id/lahanmy/
} 
Analysing the EM-DAT database, we also find a similar conclusion with regards to damaging power of droughts relative to other weather hazards. According to EMDAT, droughts occurred only 3 times in Indonesia during the 1990-2015 period ${ }^{23}$. However, the monetary damages of these events were very high. Each event, on average, cost 890 million USD or 296.67 million USD per event. We present in Annex 4 details of the impact of each type of weather shocks that occurred in the period of $1990-2017$ in Indonesia. Total economic losses from droughts, floods, landslides, and storms (excluding tsunami) during that period have almost topped US\$7.7 billion or US\$285.2 million every year (or $0.32 \%$ of average GDP of the same period).

Looking more closely at the micro level, Korkeala et al. (2009) find that delayed onset of the rainy season causes a 13 percent decline in per capita consumption of poor households ${ }^{24}$, but this reduction does not persist to the following rainy season. Maccini and Yang (2009) find that a weather shock during the first year of life could significantly affect an adult's outcomes in the future. One of their main findings is that more than normal rainfall in the very early years of women (before school entry) has a strong relationship with better health, schooling, and socioeconomic status when they are adults.

\subsection{Data and Methods}

This chapter investigates the effect of droughts on households. Since a complete historical drought disaster data at lower administrative areas (such as village or sub-district level) doesn't exit, or at least is not known publicly, we construct a drought events measure based on monthly historical weather data. In so doing, we measure the drought conditions using the Standardized Precipitation Evapotranspiration Index or SPEI (Vicente-Serrano, Beguera, \& Lpez-Moreno, 2010; Vicente-Serrano, Beguera, LpezMoreno, et al., 2010; Beguería et al., 2014; Yu et al., 2014; Stagge et al., 2015). For the household data, we use the Indonesian Family Life Surveys that record social and economic outcomes of sample households and individuals throughout Indonesia. The surveys have been conducted from 1992 (the $1^{\text {st }}$ wave) to the most recent one at the time

\footnotetext{
23 EMDAT consider severe droughts at country level, while this paper analyses presumably drought events sub-nationally disaggregated at sub-district level.

${ }^{24}$ Defined as those with average per capita consumption in the lowest quintile
} 
of writing this thesis, in 2014 (the $5^{\text {th }}$ wave). We match the location of the IFLS households, available at the sub-district level, with the drought events indictor we create.

\subsubsection{Data}

\subsubsection{Weather data and constructions of drought events}

Since it is not possible to get a complete historical weather station data covering Indonesian areas in a spatially detailed resolution, we use an index obtained from satellite observations. There are several methods for drought assessments where temperature and rainfall are commonly used weather variables for calculating the drought index. Here, we use the Standard Precipitation Evapotranspiration Index (SPEI) to determine droughts ${ }^{25}$. It is calculated using the monthly precipitation and potential evapotranspiration from the Climatic Research Unit (CRU) of the University of East Anglia (version 3.23 time series) ${ }^{26}$. We use SPEIbase v.2.5 Global 12-month gridded datasets that are constructed into a panel at the sub-district level in Indonesia.

SPEI is a relatively new drought index, following its widely used predecessor, the SPI (Standardized Precipitation Index). The new element in the SPEI is the inclusion of temperature data in addition to precipitation (Vicente-Serrano, Beguera, \& Lpez-Moreno, 2010; Stagge et al., 2015). In comparison with the Palmer Drought Severity Index (PDSI), SPEI has the advantage of being multi-scalar, which means it has time scale (between 1 and 48 months) in addition to being spatially specific (Vicente-Serrano, Beguera, LpezMoreno, et al., 2010).

Drought is one of the most difficult weather extremes to define as the occurrence of a drought event depends on the spatial and temporal situation, which makes it difficult to quantify drought severity. In this paper, we define a drought event as a condition where SPEI values ( -3 to 3 ) at the study area are below negative 1 in 6 consecutive months (within a year). Accordingly, we assign 1 for each sub-district when we considered there was a drought event in that year. We refer to SPEI data from 1990 to 2013. The theoretical basis for our assumption is based on previous works that suggest SPEI values at negative 1 spell moderate drought conditions (Yu et al., 2014; Wang et al., 2015). Motivated by the

\footnotetext{
${ }^{25}$ Besides SPEI, alternative indices use other weather measurements for examining the economic impact of drought shocks. Examples include using more weather station data or other satellites-based methods constructed indices such as the Standardized Precipitation Index (SPI), or the Palmer Drought Severity Index (PDSI). The use of these alternative indices should be considered for future research.

${ }^{26}$ See global dataset at: http://hdl.handle.net/10261/153475 (we use SPEIbase v.2.5 Global 12-month).
} 
findings of Spinoni et al. (2015) and Homdee et al. (2016), we assume only prolonged drought conditions, which in this case we chose 6 consecutive months, have a meaningful economic impact 27.

Further, we argue that adverse effects of drought may differ according to their intensity. To test that, we classify drought severity level into 3 categories: moderate, severe and extreme. Moderate drought refers to a condition where monthly drought index (SPEI values) are between -1.49 and -1 in 6 consecutive months in a year or between -1.49 and -1 in 4 consecutive rainy season months. Severe drought is defined as a condition where monthly drought index (SPEI values) are between -1.99 and -1.5 in 6 consecutive months in a year or between -1.99 and -1.5 in 4 consecutive rainy season months.

\section{Drought Index at Sub-District level Across Main Islands}

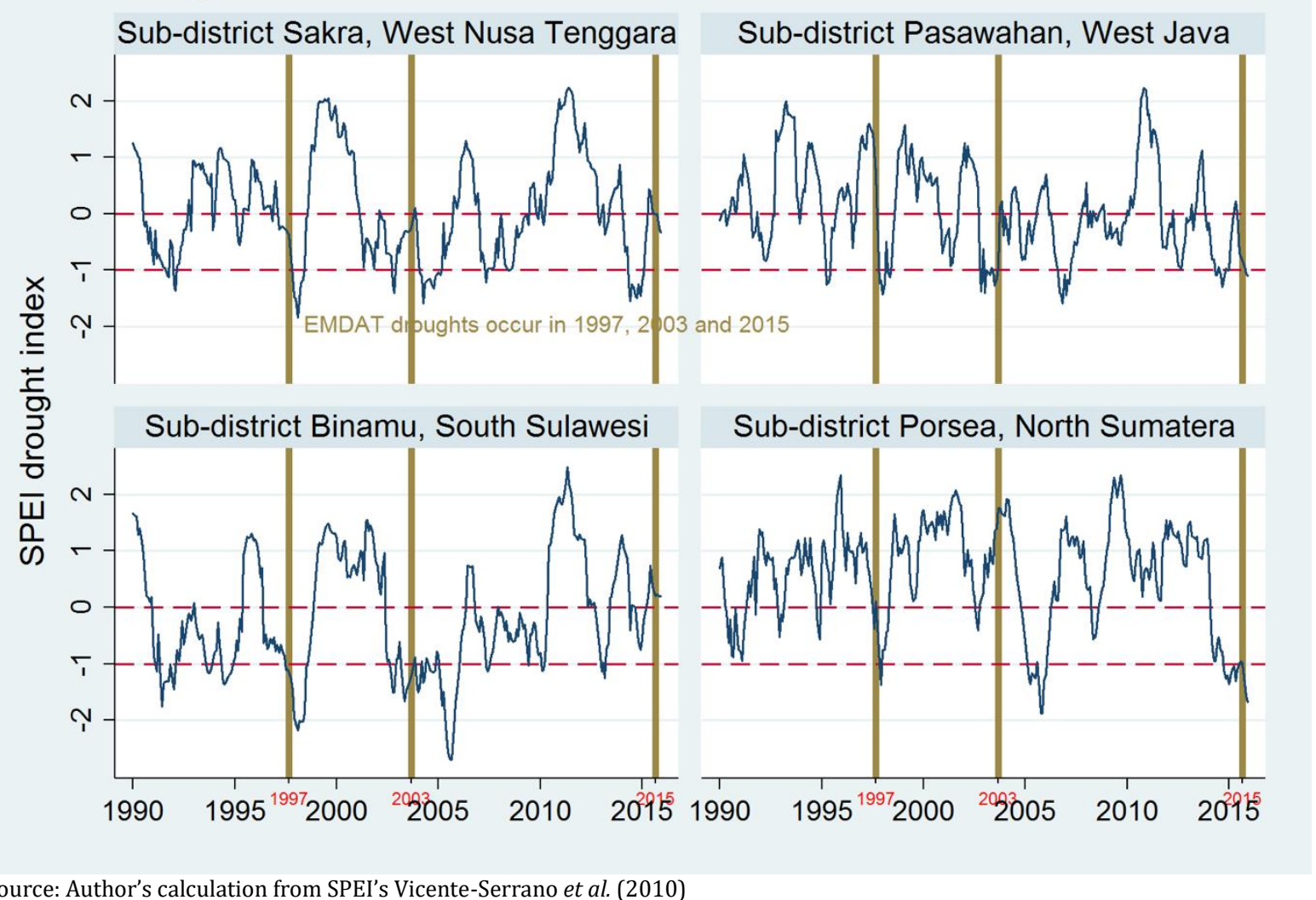

Source: Author's calculation from SPEI's Vicente-Serrano et al. (2010)

Figure 8 Drought indices based on SPEI-12 month at sub-district level

Lastly, we consider extreme drought; where monthly drought index (SPEI values) are below -2 in 6 consecutive months in a year or below -2 in 4 consecutive rainy season

\footnotetext{
27 This is an arbitrary choice, which refers to several references. Spinoni et al. (2015) assume a drought event in Europe starts when drought indices below -1 for at least 3 consecutive months. Homdee et al. (2016) found that the prolonged droughts, indicated by continuous negative values of drought indices, had adverse effects on the agricultural and water resource sectors across Thailand.
} 
months. The results are presented in Section 4.3. These threshold values are commonly used for SPI and SPEI.

We present in Figure 8 graphs of historical SPEI values at sub-districts across the main islands in Indonesia (Sumatra, Java, Nusa Tenggara, and Sulawesi). A sample of selected districts in the different islands shows the variations of the SPEI in some periods. As might have been expected, some neighbouring sub-districts have a similar pattern of drought indices as weather shock incidents can occur in large spatial areas, especially those that have similar geographical features such as altitude, proximity to water sources or oceans, and land use. We compare our SPEI values at sub-districts level with EMDAT's droughts in 1997, 2003 and 2015 in Figure 8. Our drought methodology identifies droughts at the local level. We present an example of drought history based on this paper's method in Annex 4 where the table shows droughts occurred 5 times in Binamu sub-districts (1991, 1994, 1997, 1998 and 2005), 3 times in Sakra, never in Pesawahan and 1 time in Porsea 28. These shocks may be typically local and not be reported nationally.

\subsubsection{Household longitudinal data (Indonesian Family Life Surveys/IFLS)}

The Indonesia Family Life Survey (IFLS) consists of very rich information on social and economic indicators that are collected at household and individual level such as marital status, income, expenditures, education, assets, health outcomes, labour market outcomes, etc. The first wave samples reside in 13 provinces (out of country's total of 27 provinces) representing $83 \%$ of the Indonesian population. IFLS traces the same individuals and households since the first wave and so far has achieved a high re-contact rate (92\%-94\% for households and $87 \%$ - 90\% for individual respondents). This high rate is exceptional. The high rate provides assurance for consistent analysis of the household panel data as it reduces the potential bias due to non-random attrition. Thomas et al. (2012) describe IFLS survey design and field strategies that contributed to reducing attrition rates over four waves of the survey. The IFLS5 report explains that

\footnotetext{
${ }^{28}$ We present drought events, based on our drought method, that occur in selected district areas above in Annex 5.We observe that SPEI values in 1997 in Nusa Tenggara and South Sulawesi are below -1 for several months (more than 6 months). According to our method, these indicate prolonged droughts have occurred in those areas. On the other hand, although there is a downward trend in 1997, areas in Java and Sumatera remain in neutral to mild drought condition (SPEI values are between 0 and -1 within only 2 consecutive months). As SPEI values below minus 1 were only recorded in 2 months (less than threshold 6 consecutive months), there was no drought in the sub-district of Pasawahan and Porsea in 1997. This may be related with FAO report in late April 1998 regarding the impact of 1997 drought on Indonesia. The report suggests severe drought in 1997 was widespread in some eastern regions. Total damage is 230,000 hectares of production areas of the total 14 million hectares planted areas, where most of central productions of rice in Java and Sumatera were not severely affected (see FAO's map in the report http://www.fao.org/NEWS/GLOBAL/GW9810-e.htm)
} 
there have been adjustments and corrections on sample attrition of the previous waves in order to improve the quality of IFLS longitudinal data (Strauss et al., 2016). ${ }^{29}$

To this end, we develop a household panel data consisting of demographic and economic information from each IFLS wave. As we are interested in the impact of droughts on households, we estimate the effect of drought on income and consumption. We examine total income per capita, which is a sum of profit from farm business as well as from nonfarm business, wages from the labour market and revenue from assets (selling and renting). ${ }^{30}$ Farm profit income refers to the sum of revenues minus expenses from farm business, including rental revenues from farm assets. Variable non-farm income is a sum of revenues minus expenses generated from households' own businesses, such as restaurants, real estate, construction, and services like hairdressing, taxi driving, or tailoring. In addition, we also look at the impact on incomes from selling assets. Two dependent variables are included accordingly: income from total selling of all type of assets and income from selling farm assets. We estimate the effects on the selling of farm assets to look more specifically at one potential drought effect on agricultural activity.

In the IFLS, household expenditures are categorized into 5 items; 1) food expenses 2) non-food frequently purchased goods and services 3) non-food less frequent expenses 4) education expenses and 5) housing expenditures. Food expenditure is the sum of all foodrelated consumption, such as the purchase of staples, meats, fishes, dairy products, snacks etc. Non-food frequent consumption is the sum of frequently purchased goods and services such as utilities, personal toiletries, household items, recreation and entertainment, transportation, and sweepstakes. Less frequently consumed goods and services include clothing, household supplies, furniture, medical costs, taxes and expenses for ritual/religious ceremonies. Lastly, we estimate potential drought effects on education by looking at the relationship between droughts and education expenses, which is of the sum total expenses for tuition, uniform, transportation, and boarding fees.

The unit of incomes is in Rupiah per capita in the past 12 months and the unit of expenditures is in Rupiah per capita in the month. The panel also contains household characteristics that vary for every IFLS period such as farmland, total assets, labour

\footnotetext{
${ }^{29}$ In this analysis, the sample is not split by urban and rural area. This split will be considered in future work. It is plausible that rural and urban households are affected differently, but the rural-urban distinction is not so clear-cut.

${ }^{30}$ Here, we do not specifically test drought impact on farm/rural households.
} 
income, migration (move or not from original location - at sub-district level) and household head's education background. The outcome variables are inflation-adjusted using Consumer Price Index deflators, as detailed in Strauss et al. (2002). The temporal deflators are 1996-base price (for IFLS 1 and 2 where surveys were taken before 1997/1998 economic crisis) and 2000-based price ((for IFLS 3-5 where surveys were taken after the economic crisis). To compare the values of outcome variables (in rural and non-rural or Java and non-Java), we adjust the value of the rupiah of outcome variables using spatial deflator variable that is based on Jakarta prices ${ }^{31}$.

The data capture Indonesian household's conditions between 1992 and 2013. We track and match households that are interviewed in all 5 waves. The empirical analysis in this study uses extended household based on 'original' households interviewed in IFLS 1993 that are traceable in all waves ${ }^{32}$. We also take out 54 households that we suspect as outliers. For example, we drop (observations of) households whose incomes data are significantly different with its economic profile such as its expenditures and total assets (high income but monthly expenditure very low). Eventually, we get 5,592 households as our panel entities (out of 7,224 original households that are interviewed in IFLS1) in 5 IFLS waves. The IFLS households in our panel reside in 282 sub-districts (kecamatan), 139 districts (kabupaten and kota) and 13 provinces (propinsi).

\subsubsection{Descriptive statistics}

We present descriptive statistics of all variables used in the empirical analysis in Table 6. The outcome variables are continuous and converted into logs. The main independent variables are discrete data (binary). We see similar trends in total income per capita and farm income per capita where average incomes drop in 1997/1998 survey and gradually increase in the subsequent surveys in 2000, 2007 and a very significant rise in the latest survey in 2014 .

\footnotetext{
${ }^{31}$ This is the ratio of the household location's poverty line to the Jakarta poverty line

${ }^{32}$ Kathleen Beegle et al. (2008) and Angelucci et al. (2009) suggest some empirical benefits of using extended household, that is constructed through family or kindship relations, as unit of analysis in a household study. More recently, Witoelar (2013) investigated consumption risk-sharing using IFLS data and found that extended family may be the one that decides resource allocation against consumption shock. He further suggests a panel of extended families may be better than using only a panel of "original" households when examining household consumption or income variations. Estimating the impact of weather shocks using 'original' households (surveyed in 1993) may result less robust conclusion and precision than using extended family households.
} 
In constructing the drought data for each wave, we refer to particular years that relate to each wave of the IFLS. For example, droughts in the year 1990, 1991 and 1992, 1993 are used for determining variable droughtL3, droughtL2, droughtL1, and drought in IFLS 1993 while droughts in the year 2011, 2012 and 2013 are used to determine droughtL3, droughtL2, droughtL1, and drought in IFLS 2014. Table 6 shows that all period droughts (that occurred in the same year of survey, a year ago, 2 years ago or 3 years ago) for each of the survey waves. We do not see a drought had occurred at the same year for the 1993 survey in any sub-districts, and neither did we find a drought occurred in the previous year in any sub-districts in IFLS 2000, 2007 and 2014.

Table 6 Descriptive statistics main variables of all sample IFLS households

\begin{tabular}{|c|c|c|c|c|c|}
\hline VARIABLES & $\begin{array}{l}\text { IFLS } 1 \\
\text { Mean } \\
(\mathrm{SD}) \\
\end{array}$ & $\begin{array}{l}\text { IFLS } 2 \\
\text { Mean } \\
\text { (SD) } \\
\end{array}$ & $\begin{array}{l}\text { IFLS } 3 \\
\text { Mean } \\
\text { (SD) } \\
\end{array}$ & $\begin{array}{l}\text { IFLS } 4 \\
\text { Mean } \\
(\mathrm{SD}) \\
\end{array}$ & $\begin{array}{l}\text { IFLS } 5 \\
\text { Mean } \\
(\mathrm{SD}) \\
\end{array}$ \\
\hline $\begin{array}{l}\text { OUTCOME VARIABLES: } \\
\text { pci (X1000 IDR) } \\
\text { Income per capita in the past } 12 \text { month }\end{array}$ & $\begin{array}{c}2,283 \\
(34,498)\end{array}$ & $\begin{array}{c}1,764 \\
(13,779)\end{array}$ & $\begin{array}{l}2,052 \\
(9,454)\end{array}$ & $\begin{array}{c}3,729 \\
(10,700)\end{array}$ & $\begin{array}{c}8,733 \\
(21,585)\end{array}$ \\
\hline $\begin{array}{l}\text { fipc (x1000 IDR) } \\
\text { Farm Income Per capita in the past } 12 \text { month }\end{array}$ & $\begin{array}{c}416 \\
(15,854)\end{array}$ & $\begin{array}{c}201 \\
(1,218)\end{array}$ & $\begin{array}{c}291 \\
(1,856)\end{array}$ & $\begin{array}{c}637 \\
(2,006)\end{array}$ & $\begin{array}{c}1,121 \\
(4,139)\end{array}$ \\
\hline $\begin{array}{l}\text { nfipc (x1000 IDR) } \\
\text { Non-Farm Income per capita in the past } 12 \text { month }\end{array}$ & $\begin{array}{c}123 \\
(2,787)\end{array}$ & $\begin{array}{c}412 \\
(7,960)\end{array}$ & $\begin{array}{c}146 \\
(851)\end{array}$ & $\begin{array}{c}974 \\
(4,536)\end{array}$ & $\begin{array}{c}1,889 \\
(6,825)\end{array}$ \\
\hline $\begin{array}{l}\text { farmasset (x1000 IDR) } \\
\text { Income from selling farm assets per capita in the past } 12 \text { month }\end{array}$ & $\begin{array}{c}42 \\
(456)\end{array}$ & $\begin{array}{c}36 \\
(375)\end{array}$ & $\begin{array}{c}55 \\
(675)\end{array}$ & $\begin{array}{c}72 \\
(575)\end{array}$ & $\begin{array}{c}311 \\
(2,042)\end{array}$ \\
\hline $\begin{array}{l}\text { assets_sold ( } \mathbf{x} 1000 \text { IDR) } \\
\text { Income from selling all assets per capita in the past } 12 \text { month }\end{array}$ & $\begin{array}{c}285 \\
(1,895)\end{array}$ & $\begin{array}{c}580 \\
(6,493)\end{array}$ & $\begin{array}{c}211 \\
(2,610)\end{array}$ & $\begin{array}{c}411 \\
(3,227)\end{array}$ & $\begin{array}{c}1,373 \\
(8,743)\end{array}$ \\
\hline $\begin{array}{l}\text { total expenditure (x1000 IDR) } \\
\text { Monthly total expenditure per capita }\end{array}$ & $\begin{array}{c}1,962 \\
(3,635)\end{array}$ & $\begin{array}{c}1,972 \\
(3,093)\end{array}$ & $\begin{array}{l}2,159 \\
(4,440)\end{array}$ & $\begin{array}{l}2,314 \\
(3,562)\end{array}$ & $\begin{array}{l}2,349 \\
(2,827)\end{array}$ \\
\hline $\begin{array}{l}\text { food (x1000 IDR) } \\
\text { Monthly food expenditure per capita }\end{array}$ & $\begin{array}{c}972 \\
(1,193)\end{array}$ & $\begin{array}{c}1,002 \\
(1,179)\end{array}$ & $\begin{array}{c}1,083 \\
(1,257)\end{array}$ & $\begin{array}{l}1,188 \\
(1,500)\end{array}$ & $\begin{array}{c}1,199 \\
(1,330)\end{array}$ \\
\hline $\begin{array}{l}\text { non-food (x1000 IDR) } \\
\text { Monthly non-food expenditure per capita }\end{array}$ & $\begin{array}{c}958 \\
(3,046)\end{array}$ & $\begin{array}{c}957 \\
(2,382)\end{array}$ & $\begin{array}{c}1,071 \\
(4,008)\end{array}$ & $\begin{array}{l}1,130 \\
(2,818)\end{array}$ & $\begin{array}{c}1,152 \\
(1,919)\end{array}$ \\
\hline $\begin{array}{l}\text { education (x1000 IDR) } \\
\text { Monthly education expenditure per capita }\end{array}$ & $\begin{array}{c}311 \\
(1,052)\end{array}$ & $\begin{array}{c}286 \\
(817)\end{array}$ & $\begin{array}{c}167 \\
(505)\end{array}$ & $\begin{array}{c}182 \\
(500)\end{array}$ & $\begin{array}{c}187 \\
(519)\end{array}$ \\
\hline $\begin{array}{l}\text { non-food frequent (x1000 IDR) } \\
\text { Monthly non-food frequent expenditure per capita }\end{array}$ & $\begin{array}{c}543 \\
(1,349)\end{array}$ & $\begin{array}{c}559 \\
(1,210)\end{array}$ & $\begin{array}{c}310 \\
(646)\end{array}$ & $\begin{array}{c}371 \\
(1,542)\end{array}$ & $\begin{array}{c}369 \\
(727)\end{array}$ \\
\hline $\begin{array}{l}\text { non-food less frequent (x1000 IDR) } \\
\text { Monthly non-food less frequent expenditure per capita }\end{array}$ & $\begin{array}{c}437 \\
(1,108)\end{array}$ & $\begin{array}{c}458 \\
(1,131)\end{array}$ & $\begin{array}{c}257 \\
(631)\end{array}$ & $\begin{array}{c}278 \\
(673)\end{array}$ & $\begin{array}{c}292 \\
(697)\end{array}$ \\
\hline \multicolumn{6}{|l|}{ MAIN COVARIATES; } \\
\hline $\begin{array}{l}\text { drought } \\
\text { Drought occurred at the same year (0/1) }\end{array}$ & $\begin{array}{c}0.00 \\
(0.00)\end{array}$ & $\begin{array}{c}0.13 \\
(0.34)\end{array}$ & $\begin{array}{c}0.32 \\
(0.47)\end{array}$ & $\begin{array}{c}0.02 \\
(0.15)\end{array}$ & $\begin{array}{c}0.41 \\
(0.49)\end{array}$ \\
\hline $\begin{array}{l}\text { droughtL1 } \\
\text { Drought one year before survey (0/1) }\end{array}$ & $\begin{array}{l}0.24 \\
(0.42)\end{array}$ & $\begin{array}{c}0.01 \\
(0.10)\end{array}$ & $\begin{array}{c}0.00 \\
(0.00)\end{array}$ & $\begin{array}{c}0.00 \\
(0.00)\end{array}$ & $\begin{array}{c}0.00 \\
(0.00)\end{array}$ \\
\hline $\begin{array}{l}\text { droughtL2 } \\
\text { Drought occurred two year before survey (0/1) }\end{array}$ & $\begin{array}{c}0.24 \\
(0.43)\end{array}$ & $\begin{array}{c}0.19 \\
(0.39)\end{array}$ & $\begin{array}{c}0.57 \\
(0.50)\end{array}$ & $\begin{array}{c}0.15 \\
(0.36)\end{array}$ & $\begin{array}{c}0.04 \\
(0.18)\end{array}$ \\
\hline $\begin{array}{l}\text { droughtL3 } \\
\text { Drought occurred three year before survey (0/1) }\end{array}$ & $\begin{array}{c}0.16 \\
(0.37)\end{array}$ & $\begin{array}{c}0.20 \\
(0.40)\end{array}$ & $\begin{array}{c}0.32 \\
(0.47)\end{array}$ & $\begin{array}{c}0.08 \\
(0.27)\end{array}$ & $\begin{array}{c}0.00 \\
(0.00)\end{array}$ \\
\hline \multicolumn{6}{|l|}{ CONTROL VARIABLES: } \\
\hline $\begin{array}{l}\text { hhsize } \\
\text { Number of household's member (person) }\end{array}$ & $\begin{array}{c}4.80 \\
(2.09)\end{array}$ & $\begin{array}{c}5.21 \\
(2.54)\end{array}$ & $\begin{array}{c}6.67 \\
(3.90)\end{array}$ & $\begin{array}{c}7.97 \\
(4.85)\end{array}$ & $\begin{array}{c}9.52 \\
(5.99)\end{array}$ \\
\hline $\begin{array}{l}\text { farmland } \\
\text { Own land for farming }(0 / 1)\end{array}$ & $(0.50)$ & $\begin{array}{c}0.50 \\
(0.50)\end{array}$ & $\begin{array}{c}0.48 \\
(0.50)\end{array}$ & $\begin{array}{c}0.45 \\
(0.50)\end{array}$ & $\begin{array}{c}0.48 \\
(0.50)\end{array}$ \\
\hline ownfarm & 0.15 & 0.19 & 0.29 & 0.39 & 0.47 \\
\hline
\end{tabular}




\begin{tabular}{lccccc} 
Own farm asset>= IDR 10 million (0/1) & $(0.35)$ & $(0.39)$ & $(0.46)$ & $(0.49)$ & $(0.50)$ \\
richasset & 0.07 & 0.17 & 0.08 & 0.13 & 0.30 \\
Total all assets $>=100$ Million (0/1) & $(0.25)$ & $(0.37)$ & $(0.27)$ & $(0.33)$ & $(0.46)$ \\
labour & 0.13 & 0.20 & 0.14 & 0.25 & 0.41 \\
HH receives income from employment $>$ 1million/year $(0 / 1)$ & $(0.33)$ & $(0.40)$ & $(0.34)$ & $(0.43)$ & $(0.49)$ \\
ageHead & 44.86 & 47.87 & 50.52 & 53.28 & 55.62 \\
Age of Household's head (year) & $(13.48)$ & $(12.88)$ & $(12.43)$ & $(11.39)$ & $(10.84)$ \\
head_man & 0.86 & 0.84 & 0.84 & 0.80 & 0.78 \\
Household head is male (0/1) & $0.34)$ & $(0.36)$ & $(0.37)$ & $(0.40)$ & $(0.41)$ \\
univ & 0.03 & 0.02 & 0.05 & 0.10 & 0.13 \\
Household head attended university (0/1) & $(0.17)$ & $(0.15)$ & $(0.22)$ & $(0.30)$ & $(0.34)$ \\
hischool & 0.12 & 0.05 & 0.11 & 0.20 & 0.23 \\
Household head attended highs school (0/1) & $(0.33)$ & $(0.21)$ & $(0.32)$ & $(0.40)$ & $(0.42)$ \\
juniorhi & 0.20 & 0.07 & 0.15 & 0.24 & 0.28 \\
Household head attended junior high $(0 / 1)$ & $(0.40)$ & $(0.25)$ & $(0.35)$ & $(0.43)$ & $(0.45)$ \\
element & 0.52 & 0.12 & 0.21 & 0.30 & 0.31 \\
Household head attended elementary $(0 / 1)$ & $(0.50)$ & $(0.32)$ & $(0.41)$ & $(0.46)$ & $(0.46)$ \\
literate & 0.70 & 0.68 & 0.79 & 0.87 & 0.91 \\
Household head can read and write $(0 / 1)$ & $(0.46)$ & $(0.47)$ & $(0.40)$ & $(0.34)$ & $(0.29)$ \\
\hline Source: Author's calculation from the Indonesian Family Life Survey $(I F L S) 1-5(1993,1997,2000,2007,2015)$. & &
\end{tabular}

\subsubsection{Empirical methods}

We link the spatiotemporal drought indices with household longitudinal survey data. As we are interested in investigating the effect of that risk on a group of inhabitants (a household) that experience the same shocks, we exploit the panel dimension of our data using household-effect to avoid a problem of selection in the treatment (due to unobservable household characteristics that are constant over time). Further, we control for observable household characteristics such as if the household has ever moved before the survey outside the sub-district, if it owns farm assets, and if a household member receives income from labour wages, etc.

\subsubsection{Households and time fixed-effects}

We employ Hausman tests to choose between fixed-effect (FE) and random-effect (RE) for our model specification. The results are statistically significant, which suggest the FE model is more appropriate than the RE model because unobserved household characteristics (error terms) appear to be correlated with the independent variables. More descriptions regarding the Hausman tests and variance decompositions of the main explanatory variables are presented in the Results section. Finally, we consider time fixed-effect in our model to eliminate the effect of other non-drought events in our investigation on the variability of household outcomes. To test whether a time effect control is needed, we perform the Wald test (as well as the F-test) on all income and expenditure models. The results indicate that the coefficients for all waves of the survey 
are jointly not equal to zero, which suggests we should include time fixed-effects in the regression.

Hence, our fixed-effect model can be written as follows:

$$
\log \left(Y_{i, s, t)}=\sum_{l=0}^{3} \beta_{l} \text { Drought }_{s, t-l}+\sum_{k} \theta_{k} X_{i, k, t}+\alpha_{i}+\delta_{t}+\varepsilon_{i t}\right.
$$

where $\boldsymbol{Y}_{\boldsymbol{i}, \boldsymbol{s}, \boldsymbol{t}}$ represents a set of household outcomes per capita of household $\boldsymbol{i}$ at subdistrict $\boldsymbol{s}$ at IFLS time $\boldsymbol{t}$. We use natural log of the outcomes for easy interpretation of the effects. The $\boldsymbol{\beta}_{\boldsymbol{L}}$ denotes the effect of Drought that occurred in sub-district $\boldsymbol{S}$ at $\boldsymbol{l}$ year prior to the $\boldsymbol{t}$ survey, where $\boldsymbol{l} \in$ the $(0,1,2,3)$ and $t \in(1993,1997,2000,2007,2014)$. Vector $\boldsymbol{X}_{\boldsymbol{i}, \boldsymbol{k}, \boldsymbol{t}}$ represents time-variant observable characteristics such as whether household own farm land or not, receive labour wage, age and education background of the head of households, etc. We control for these time-varying observable characteristics to get more precise estimation and control for missing variables bias. The $\boldsymbol{\alpha}$ denotes household fixed effects and $\boldsymbol{\delta}$ represent time-effect and $\boldsymbol{\varepsilon}$ denotes time-varying residuals across panel entities. We apply robust standard error methods for better precision, in which we elaborate the chosen method in the next section.

\subsubsection{Robust standard errors for cross-sectional dependence and heteroscedasticity}

Panel data analysis obviously offers several advantages relative to cross-sectional, such as more accurate inference of model parameters, control the impact of omitted variable and uncover dynamics relationships over time (Hsiao, 2014). However, panel data has intrinsically a major issue; that is the individual entities may be correlated or interdependent, which potentially bias parameter estimations and thus casual inference. There are several propositions in the econometric literature when we suspect errors in our panel models are not i.i.d as they correlate with each other across entities or serially in some unknown way. Many recommend to apply robust standard errors techniques (Driscoll \& Kraay, 1998; Kezdi, 2003; Baltagi \& Hashem Pesaran, 2007; Sarafidis \& Wansbeek, 2012; Pesaran, 2015; Born \& Breitung, 2016), while some others suggest more complex methods using spatial regression techniques, which allow both spatial dependence and heterogeneity.

Our panel data consist of drought events, which is constructed from weather data at the sub-district level, and household economic outcomes data from IFLS. As a result, there 
are some IFLS household respondents reside at the same sub-district so they have similar drought experience and these cases are more common in the densely populated IFLS areas, like Java. This data construction features problem of cross-sectional dependencies that may potentially bias the estimation. Further, our statistical checks show the likelihood of correlation of the residuals across panel entities. To address these issues, we apply the robust standard error method of Driscoll and Kraay (D\&K SE) to improve the precision of the estimation. The D\&K SE is suitable when cross-sectional dependence is detected and specifically useful for panel regressions (Driscoll \& Kraay, 1998; Hoechle, 2007). An additional feature of this technique, similar to other robust standard error techniques, is that it also deals with heteroscedasticity in the outcomes and predictors.

\subsection{Results and discussion}

We perform statistical checks to test the unbiasedness of our model. Further, we also check in the models whether there are enough variations in the dependent variable explained by the changes in the predictors of interest using variance decomposition. The statistical check tests whether, in fitted models, variations of the predictors (in this case droughts) across wave of the survey (time-series) are higher than across panel entity (household), which is true in this case. The test helps to determine whether drought episodes as the main independent variables of interest specified in the models are good predictors for estimating changes in the outcomes. If the results are not convincing (fewer variations in time series droughts as opposed to cross-section ones), the models may not be meaningful to infer a causal relationship between droughts and the outcomes (incomes and expenditures). The summary of the tests is presented in Table 7 (a detailed version can be obtained upon request). Annex 6 and 7 present graphs of the statistical tests.

Table 7 Summary of statistical checks

\begin{tabular}{|c|c|c|c|c|}
\hline & Test type & Result & Benchmark & Concluding notes \\
\hline \multirow[t]{2}{*}{$\begin{array}{l}\text { Multicollinearity } \\
\text { test }\end{array}$} & VIF test & $\begin{array}{l}\text { Mean VIF } \\
=2.8\end{array}$ & $\begin{array}{l}\text { No } \\
\text { collinearity } \\
\text { if value }<10 \\
\end{array}$ & no collinearity between predictors \\
\hline & Pearson Corr. & $\begin{array}{l}\text { Coef. range } \\
=0.04- \\
0.46\end{array}$ & $\begin{array}{l}\text { No } \\
\text { collinearity } \\
\text { if value }<0.9 \\
\end{array}$ & $\begin{array}{l}\text { all predictors are hardly related to } \\
\text { each other }\end{array}$ \\
\hline $\begin{array}{l}\text { Heteroskedasticity } \\
\text { test }\end{array}$ & Wald test & $\begin{array}{l}\text { Prob }>\text { chi2 } \\
=0.0000\end{array}$ & $\begin{array}{l}\text { Significant } \\
\text { if value }< \\
0.05 \\
\end{array}$ & $\begin{array}{l}\text { heteroskedasticity is present in the } \\
\text { model }\end{array}$ \\
\hline
\end{tabular}




\begin{tabular}{|c|c|c|c|c|}
\hline & $\begin{array}{l}\text { Breusch-Pagan } \\
\text { test }\end{array}$ & $\begin{array}{l}\text { Prob }>\text { chi2 } \\
=0.0000\end{array}$ & $\begin{array}{l}\text { Significant } \\
\text { if value < } \\
0.05\end{array}$ & presence of heteroscedasticity \\
\hline Autocorrelation & $\begin{array}{l}\text { Wooldridge } \\
\text { test for } \\
\text { autocorrelation } \\
\text { in panel data }\end{array}$ & $\begin{array}{l}\text { Prob }>\text { F = } \\
0.0000\end{array}$ & $\begin{array}{l}\text { Significant } \\
\text { if value < } \\
0.05\end{array}$ & $\begin{array}{l}\text { Results suggest to rejects null } \\
\text { hyphotesis no autocorrelation, } \\
\text { Therefore, we conclude the data have } \\
\text { first-order autocorrelation. Higher } \\
\text { orders may have autocorrelation as } \\
\text { well, but here we didn't test for them. }\end{array}$ \\
\hline $\begin{array}{l}\text { Fixed-Effect or } \\
\text { Random-Effect }\end{array}$ & Hausman test & $\begin{array}{l}\text { Prob }>\text { chi2 } \\
=0.0000\end{array}$ & $\begin{array}{l}\text { Significant } \\
\text { if value }< \\
0.05\end{array}$ & $\begin{array}{l}\text { All model specifications (SPEI and } \\
\text { PDSI droughts) reject random } \\
\text { assumption. Therefore, fixed-effect is } \\
\text { more consistent than random-effect }\end{array}$ \\
\hline $\begin{array}{l}\text { Time fixed-effect } \\
\text { test }\end{array}$ & $\begin{array}{l}\text { Wald test } \\
\text { (testparm) }\end{array}$ & $\begin{array}{l}\text { All FE } \\
\text { models } \\
\text { have } \\
\text { results: } \\
\text { Prob>F = } \\
0.0000\end{array}$ & $\begin{array}{l}\text { Significant } \\
\text { if value }< \\
0.05\end{array}$ & $\begin{array}{l}\text { We need to control time fixed-effect } \\
\text { as there might be variations in } \\
\text { variables that are affected by other } \\
\text { special events than droughts across } \\
\text { IFLS waves }\end{array}$ \\
\hline $\begin{array}{l}\text { Variance } \\
\text { decomposition test }\end{array}$ & $\begin{array}{l}\text { Observe } \\
\text { standard } \\
\text { deviation } \\
\text { values within } \\
\text { and between }\end{array}$ & $\begin{array}{l}\text { all mean SD } \\
\text { drought } \\
\text { variables } \\
\text { across time } \\
\text { is higher } \\
\text { than across } \\
\text { households }\end{array}$ & $\begin{array}{l}\text { variances } \\
\text { of } \\
\text { explanatory } \\
\text { variables } \\
\text { within > } \\
\text { between }\end{array}$ & $\begin{array}{l}\text { drought variations across IFLS waves } \\
\text { (time-series: within) are higher than } \\
\text { variations across households (cross- } \\
\text { section: between), therefore we } \\
\text { conclude the analysis is meaningful } \\
\text { as there are enough variations to } \\
\text { identify the parameters associated } \\
\text { with the observed explanatory } \\
\text { variables (droughts) }\end{array}$ \\
\hline
\end{tabular}

\subsubsection{The Effect of weather shocks on the household economy}

\subsubsection{Impact on household incomes in the past 12 months}

The regression estimates in Table 8 indicate that there are statistically significant relationships between drought episodes and variations in household's incomes. The drought effects vary on one income type and another, and the significances depend on the drought's timing. For example, the impacts on incomes from the farm and non-farm businesses are evidently observable only in the year following a drought occurs while on incomes from selling farm assets, significant effects are observable from the present year to two years a drought occurs (see column Farm Income, Non-Farm Income and Selling Farm Assets). Moreover, a significant impact on total income is apparent only in 3 years after a drought occurred (see column Total Income, row Drought $t_{3}$ ).

Here, the adverse effects of drought are shown in different signs of the relationships with respect to income's type. The negative coefficients in total, farm, and non-farm income clearly reflect detrimental impacts of drought that cut these annual profits (see column Farm Income, Non-Farm Income, and Total Income). On the other hand, we interpret the 
positive coefficients in incomes from selling assets as negative effects because the increased incomes suggest surviving mechanisms to respond to weather shock (see column Selling Farm Assets and Selling All Type of Assets). To this end, the results suggest large and lasting effects on farm-related assets (from $11.4 \%$ to $42.1 \%$, see column Selling Farm Assets). This is probably not surprising because selling direct farm assets like livestock, plants, equipment (including vehicles) and farm land may be the easiest way of getting cash during economic hardship that affects incomes from the production activities.

Table 8 Effects of drought shocks on household incomes

\begin{tabular}{|c|c|c|c|c|c|}
\hline & \multicolumn{5}{|c|}{ DEPENDENT VARIABLE: HOUSEHOLD INCOMES (log) } \\
\hline & $\begin{array}{l}\text { Total } \\
\text { Income }\end{array}$ & $\begin{array}{l}\text { Farm } \\
\text { Income }\end{array}$ & $\begin{array}{l}\text { Non-Farm } \\
\text { Income }\end{array}$ & $\begin{array}{l}\text { Selling Farm } \\
\text { Assets }\end{array}$ & $\begin{array}{c}\text { Selling All } \\
\text { Type of } \\
\text { Assets } \\
\end{array}$ \\
\hline Drought $t_{0}(1 / 0)$ & $\begin{array}{c}0.0307 \\
(0.0297)\end{array}$ & $\begin{array}{c}0.0122 \\
(0.0226)\end{array}$ & $\begin{array}{l}-0.0094 \\
(0.0240)\end{array}$ & $\begin{array}{l}0.1139^{* *} \\
(0.0346)\end{array}$ & $\begin{array}{c}0.0243 \\
(0.0415)\end{array}$ \\
\hline Drought $t_{1}(1 / 0)$ & $\begin{array}{c}-0.0403 \\
(0.0153)\end{array}$ & $\begin{array}{c}-0.0711^{* *} \\
(0.0116)\end{array}$ & $\begin{array}{c}-0.2327^{* * *} \\
(0.0197)\end{array}$ & $\begin{array}{l}0.2962^{* * *} \\
(0.0469)\end{array}$ & $\begin{array}{c}-0.0515 \\
(0.0551)\end{array}$ \\
\hline Drought $t_{2}(1 / 0)$ & $\begin{array}{c}-0.0389 \\
(0.0192)\end{array}$ & $\begin{array}{c}0.0446 \\
(0.0512)\end{array}$ & $\begin{array}{l}-0.0906 \\
(0.0480)\end{array}$ & $\begin{array}{l}0.4231^{* * *} \\
(0.0864)\end{array}$ & $\begin{array}{l}0.2174^{* * *} \\
(0.0384)\end{array}$ \\
\hline Drought $t_{3}(1 / 0)$ & $\begin{array}{c}-0.0408^{* *} \\
(0.0079)\end{array}$ & $\begin{array}{l}-0.0206 \\
(0.0231)\end{array}$ & $\begin{array}{l}-0.1355 \\
(0.0527)\end{array}$ & $\begin{array}{c}-0.0543 \\
(0.0341)\end{array}$ & $\begin{array}{c}-0.1314^{* *} \\
(0.0399)\end{array}$ \\
\hline Household size & $\begin{array}{c}-0.0513^{* * *} \\
(0.0047)\end{array}$ & $\begin{array}{c}-0.0506^{* * *} \\
(0.0032)\end{array}$ & $\begin{array}{c}-0.0739^{* * *} \\
(0.0071)\end{array}$ & $\begin{array}{c}-0.0696^{* * *} \\
(0.0115)\end{array}$ & $\begin{array}{c}-0.0767^{* * *} \\
(0.0115)\end{array}$ \\
\hline Move out & $\begin{array}{c}0.0487 \\
(0.0229)\end{array}$ & $\begin{array}{l}-0.0479^{* *} \\
(0.0125)\end{array}$ & $\begin{array}{c}0.0444 \\
(0.0267)\end{array}$ & $\begin{array}{c}0.1974 \\
(0.1209)\end{array}$ & $\begin{array}{c}0.0496 \\
(0.0372)\end{array}$ \\
\hline Own a farmland & $\begin{array}{c}0.0544 \\
(0.0540)\end{array}$ & $\begin{array}{l}-0.0756 \\
(0.0453)\end{array}$ & $\begin{array}{c}0.0035 \\
(0.0081)\end{array}$ & $\begin{array}{c}-0.0956 \\
(0.0624)\end{array}$ & $\begin{array}{l}-0.0808^{*} \\
(0.0374)\end{array}$ \\
\hline Run a farm & $\begin{array}{l}0.3127^{* * *} \\
(0.0332)\end{array}$ & $\begin{array}{l}0.4959^{* * *} \\
(0.0393)\end{array}$ & $\begin{array}{l}-0.0198 \\
(0.0100)\end{array}$ & $\begin{array}{l}0.4784^{* * *} \\
(0.0405)\end{array}$ & $\begin{array}{l}0.2707^{* *} \\
(0.0627)\end{array}$ \\
\hline Rich Assets & $\begin{array}{l}0.3475^{* * *} \\
(0.0338)\end{array}$ & $\begin{array}{l}0.3239^{* * *} \\
(0.0245)\end{array}$ & $\begin{array}{l}0.2776^{* * *} \\
(0.0112)\end{array}$ & $\begin{array}{l}0.4189^{* *} \\
(0.0951)\end{array}$ & $\begin{array}{l}0.3470^{* * *} \\
(0.0527)\end{array}$ \\
\hline Receive wage & $\begin{array}{l}0.6734^{* * *} \\
(0.1215)\end{array}$ & $\begin{array}{l}0.4621^{* * *} \\
(0.0285)\end{array}$ & $\begin{array}{l}0.6783^{* * *} \\
(0.0775)\end{array}$ & $\begin{array}{l}0.5072^{* * *} \\
(0.0530)\end{array}$ & $\begin{array}{c}0.2484^{* *} \\
(0.0561)\end{array}$ \\
\hline $\begin{array}{l}\text { Economic conditions } \\
\text { (monthly expenditre) }\end{array}$ & $\begin{array}{l}0.0716^{* * *} \\
(0.0060)\end{array}$ & $\begin{array}{c}0.0305^{*} \\
(0.0140)\end{array}$ & $\begin{array}{c}0.0412^{*} \\
(0.0157)\end{array}$ & $\begin{array}{l}-0.2057^{* * *} \\
(0.0177)\end{array}$ & $\begin{array}{c}0.0850^{*} \\
(0.0354)\end{array}$ \\
\hline Age of head & $\begin{array}{l}0.0029^{* *} \\
(0.0007)\end{array}$ & $\begin{array}{l}-0.0012^{* *} \\
(0.0003)\end{array}$ & $\begin{array}{l}-0.0053^{* * *} \\
(0.0009)\end{array}$ & $\begin{array}{c}0.0043^{*} \\
(0.0019)\end{array}$ & $\begin{array}{l}-0.0011 \\
(0.0017)\end{array}$ \\
\hline Head's gender & $\begin{array}{c}0.0004 \\
(0.0412)\end{array}$ & $\begin{array}{c}0.0119 \\
(0.0156)\end{array}$ & $\begin{array}{l}-0.1238^{* *} \\
(0.0326)\end{array}$ & $\begin{array}{c}0.2485 \\
(0.1720)\end{array}$ & $\begin{array}{c}-0.0052 \\
(0.0789)\end{array}$ \\
\hline Educ (Uni) & $\begin{array}{l}-0.0354 \\
(0.0258)\end{array}$ & $\begin{array}{l}-0.1199^{* *} \\
(0.0267)\end{array}$ & $\begin{array}{c}0.0151 \\
(0.0151)\end{array}$ & $\begin{array}{c}0.0039 \\
(0.0999)\end{array}$ & $\begin{array}{l}-0.0918^{* *} \\
(0.0323)\end{array}$ \\
\hline Educ (High) & $\begin{array}{l}-0.0486 \\
(0.0277)\end{array}$ & $\begin{array}{c}0.0043 \\
(0.0272)\end{array}$ & $\begin{array}{l}-0.1160^{* *} \\
(0.0377)\end{array}$ & $\begin{array}{c}0.1685 \\
(0.0973)\end{array}$ & $\begin{array}{l}-0.0077 \\
(0.0477)\end{array}$ \\
\hline Educ (Junior) & $\begin{array}{l}0.0635^{* * *} \\
(0.0109)\end{array}$ & $\begin{array}{c}0.0072 \\
(0.0356)\end{array}$ & $\begin{array}{l}0.0846^{* *} \\
(0.0266)\end{array}$ & $\begin{array}{l}-0.0332 \\
(0.1714)\end{array}$ & $\begin{array}{c}0.0731 \\
(0.0709)\end{array}$ \\
\hline Educ (Elementary) & 0.0412 & -0.0087 & 0.0601 & -0.0868 & $-0.0752^{*}$ \\
\hline
\end{tabular}




\begin{tabular}{lccccc} 
& $(0.0356)$ & $(0.0403)$ & $(0.0510)$ & $(0.1531)$ & $(0.0350)$ \\
Can read and write & $0.1721^{* * *}$ & 0.0444 & 0.0479 & 0.0756 & 0.1413 \\
& $(0.0267)$ & $(0.0223)$ & $(0.0477)$ & $(0.1836)$ & $(0.0804)$ \\
\hline $\mathrm{N}$ & 23,626 & 12,511 & 10,929 & 2,638 & 7,443 \\
Adjusted $R^{2}$ & 0.5041 & 0.3657 & 0.2778 & 0.1363 & 0.1677 \\
\hline
\end{tabular}

Note: Driscoll and Kraay standard errors (in parentheses) are robust to heteroscedasticity and potential bias (due to error's spatial and temporal dependences). ${ }^{*} p<0.10,{ }^{* *} p<0.05,{ }^{* * *} p<0.01$ denote statistical significance for the relationship. Drought at IFLS year refers to droughts that occurred in 1993, 1997, 2000, 2007 and 2014. Drought (t-1) refers to droughts that occurred in 1992, 1996, 1999, 2006 and 2013. Drought (t-2) refers to droughts that occurred in 1991, 1995, 1998, 2005 and 2012. Drought (t-3) refers to droughts that occurred in 1990, 1994, 1997, 2004 and 2011. Models are panel household and time fixed-effects.

Overall, the results suggest the consequences of drought episodes seem to be very substantial on incomes from running own business (farm or non-farm). A drought occurred a year ago would predict a 7.11\% drop on this year's farm income and a large cut on non-farm income (up to $23.27 \%$ ). The estimated impact on income from non-farm business interestingly is much bigger than the impact on income from business beyond agriculture, which seems counter-intuitive as the primary sector depends highly on weather conditions. There could be several reasons behind this result, including a possible link between farm and non-farm business, especially in rural area contexts. In our IFLS sample, $58 \%$ of households live in rural areas, and the proportion of households receive non-farm income in rural areas is slightly higher than in urban areas $152 \%$ in rural and 48\% in urban). It shows how important non-farming livelihoods in rural areas. The linkage between farm and non-farm activities has been well documented in the previous studies, such as in Reardon et al. (1998) and in Lanjouw and Lanjouw (2001). Overall, these findings suggest entrepreneurship-style activities could be highly vulnerable in the event of drought shocks.

\subsubsection{Impact on household expenditure}

We report the summary of the regression results of expenditure models in Table 9. Our coefficient estimates show mixed relationships between drought and monthly household expenditure but the findings clearly support the short-term effects of drought on household consumption. The effects seem to fade away after 3 years.

Table 9 Effects of drought shocks on household expenditures

\begin{tabular}{lllllll}
\hline & \multicolumn{5}{c}{ DEPENDENT VARIABLE HOUSEHOLD EXPENDITURES (log) } \\
\cline { 2 - 7 } & $\begin{array}{c}\text { Total } \\
\text { Expenditure }\end{array}$ & $\begin{array}{c}\text { Food } \\
\text { Consumption }\end{array}$ & $\begin{array}{c}\text { Non Food } \\
\text { Consumption }\end{array}$ & $\begin{array}{c}\text { Education } \\
\text { Expense }\end{array}$ & $\begin{array}{c}\text { Non-Food } \\
\text { Frequent }\end{array}$ & $\begin{array}{c}\text { Non-Food } \\
\text { Less }\end{array}$ \\
\hline Drought $t_{0}(1 / 0)$ & $0.0146^{*}$ & $0.0143^{*}$ & $0.0173^{*}$ & 0.0023 & 0.0206 & $0.0351^{*}$ \\
& $(0.0051)$ & $(0.0050)$ & $(0.0052)$ & $(0.0049)$ & $(0.0192)$ & $(0.0103)$ \\
Drought $t_{1}(1 / 0)$ & $-0.0259^{*}$ & $-0.0181^{*}$ & -0.0256 & $-0.0873^{* *}$ & $-0.0474^{*}$ & 0.0110 \\
& $(0.0080)$ & $(0.0048)$ & $(0.0099)$ & $(0.0161)$ & $(0.0106)$ & $(0.0094)$ \\
Drought $t_{2}(1 / 0)$ & -0.0072 & $-0.0074^{*}$ & -0.0013 & 0.0186 & 0.0099 & 0.0228
\end{tabular}




\begin{tabular}{|c|c|c|c|c|c|c|}
\hline & $(0.0044)$ & $(0.0024)$ & $(0.0080)$ & $(0.0141)$ & $(0.0076)$ & $(0.0239)$ \\
\hline \multirow[t]{2}{*}{ Drought $t_{3}(1 / 0)$} & 0.0023 & 0.0006 & 0.0086 & -0.0318 & -0.0176 & -0.0090 \\
\hline & $(0.0027)$ & $(0.0018)$ & $(0.0056)$ & $(0.0158)$ & $(0.0143)$ & $(0.0069)$ \\
\hline \multirow[t]{2}{*}{ Household size } & $-0.0287^{* * *}$ & $-0.0362^{* * *}$ & $-0.0217^{* * *}$ & -0.0040 & 0.0011 & $-0.0051^{* * *}$ \\
\hline & $(0.0021)$ & 0.00 & $(0.0026)$ & $(0.0030)$ & $(0.0013)$ & $(0.0010)$ \\
\hline \multirow[t]{2}{*}{ Move out } & 0.0038 & 0.0060 & 0.0064 & 0.0449 & 0.0141 & -0.0033 \\
\hline & $(0.0041)$ & $(0.0093)$ & $(0.0047)$ & $(0.0279)$ & $(0.0075)$ & $(0.0116)$ \\
\hline \multirow[t]{2}{*}{ Own a farmland } & $-0.0068^{* * *}$ & -0.0045 & $-0.0164^{* *}$ & 0.0067 & 0.0028 & 0.0057 \\
\hline & $(0.0009)$ & $(0.0026)$ & $(0.0036)$ & $(0.0172)$ & $(0.0097)$ & $(0.0132)$ \\
\hline \multirow[t]{2}{*}{ Run a farm } & -0.0028 & -0.0070 & 0.0117 & 0.0253 & -0.0120 & -0.0103 \\
\hline & $(0.0050)$ & $(0.0075)$ & $(0.0058)$ & 89) & 17) & 098) \\
\hline \multirow[t]{2}{*}{ Rich Assets } & 0.0059 & 0.0023 & 0.0124 & $-0.0520^{*}$ & 0.0298 & 0.0128 \\
\hline & $(0.0083)$ & $(0.0105)$ & $(0.0091)$ & $(0.0202)$ & 195) & $(0.0233)$ \\
\hline \multirow[t]{2}{*}{ Receive wage } & -0.0039 & 0.0049 & $-0.0202^{* * *}$ & $-0.0130^{* *}$ & 0.0062 & -0.0142 \\
\hline & $(0.0068)$ & $(0.0095)$ & $(0.0031)$ & $(0.0040)$ & 086) & $(0.0150)$ \\
\hline \multirow[t]{2}{*}{ Economic conditions } & $0.0518^{* * *}$ & $0.0348^{* * *}$ & $0.0668^{* * *}$ & $0.0905^{* * *}$ & $0.1500^{* * *}$ & $0.0943^{* * *}$ \\
\hline & $(0.0122)$ & $(0.0133)$ & $(0.0123)$ & $(0.0368)$ & $(0.0385)$ & $(0.0322)$ \\
\hline \multirow[t]{2}{*}{ Age of head } & 0.0001 & 0.0003 & $-0.0003^{* *}$ & 0.0001 & $-0.0008^{* * *}$ & $-0.0011^{* * *}$ \\
\hline & $(0.0001)$ & $(0.0002)$ & $(0.0001)$ & $(0.0005)$ & $(0.0002)$ & $(0.0002)$ \\
\hline \multirow[t]{2}{*}{ Head's gender } & $-0.0372^{* * *}$ & $-0.0379^{* * *}$ & $-0.0305^{* * *}$ & $-0.0898^{* * *}$ & $-0.0206^{*}$ & -0.0077 \\
\hline & $(0.0047)$ & $(0.0041)$ & $(0.0033)$ & $(0.0152)$ & $(0.0097)$ & $(0.0113)$ \\
\hline \multirow[t]{2}{*}{ Educ (Uni) } & -0.0128 & -0.0085 & -0.0145 & 0.0020 & 0.0021 & -0.0104 \\
\hline & $(0.0098)$ & & & & $(0.0212)$ & $(0.0294)$ \\
\hline \multirow[t]{2}{*}{ Educ (High) } & 0.0099 & 0.0177 & -0.0018 & $-0.0609^{*}$ & -0.0332 & -0.0073 \\
\hline & $(0.0098)$ & $(0.0083)$ & $(0.0127)$ & $(0.0235)$ & $(0.0375)$ & $(0.0236)$ \\
\hline \multirow[t]{2}{*}{ Educ (Junior) } & $0.0222^{*}$ & 0.0184 & $0.0269^{*}$ & $0.1422^{* * *}$ & 0.0619 & 0.0083 \\
\hline & $(0.0081)$ & $(0.0096)$ & $(0.0123)$ & $(0.0224)$ & $(0.0413)$ & $(0.0156)$ \\
\hline \multirow[t]{2}{*}{ Educ (Elemen) } & -0.0109 & -0.0006 & -0.0206 & $-0.0820^{* *}$ & -0.0051 & -0.0126 \\
\hline & $(0.0071)$ & $(0.0091)$ & $(0.0118)$ & $(0.0183)$ & $(0.0128)$ & $(0.0189)$ \\
\hline \multirow[t]{2}{*}{ Can read and write } & $-0.0258^{* *}$ & $-0.0206^{* * *}$ & $-0.0293^{*}$ & -0.0136 & $-0.0362^{*}$ & -0.0303 \\
\hline & $(0.0060)$ & $(0.0041)$ & $(0.0132)$ & $(0.0152)$ & $(0.0132)$ & $(0.0158)$ \\
\hline$N$ & 27,945 & 27,666 & 27551 & 18,145 & 27,663 & 27,635 \\
\hline Adjusted $R^{2}$ & 0.7926 & 0.7164 & 0.7303 & 0.4973 & 0.6226 & 0.5823 \\
\hline
\end{tabular}

Note: Driscoll and Kraay standard errors (in parentheses) are robust to heteroscedasticity and potential bias (due to error's spatial and temporal dependences). ${ }^{*} p<0.10,{ }^{* *} p<0.05,{ }^{* * *} p<0.01$ denote statistical significance for the relationship. Drought at IFLS year refers to droughts that occurred in 1993, 1997, 2000, 2007 and 2014. Drought (t-1) refers to droughts that occurred in 1992, 1996, 1999, 2006 and 2013. Drought (t-2) refers to droughts that occurred in 1991, 1995, 1998, 2005 and 2012. Drought (t-3) refers to droughts that occurred in 1990, 1994, 1997, 2004 and 2011. Models are panel household and time fixed-effects.

Table 9 reports that a contemporaneous drought is associated with a small increase $(1.5 \%-3.5 \%)$ of monthly expenditures per capita. A year after a drought occurred, however, we find households that experience the drought reduce their expenditure compared to households that do not experience the drought. The significant results of this drought are shown in the impact on total monthly expenditure as well as on expenses for food, non-food, and education. The estimated declines range between $1.8 \%$ and $8.7 \%$, whereby spending on education is the one that is affected the most.

The estimated effects on food consumption start from the year a drought occurred until 2 years later, but it is positive at first, and only then turns negative for the following two 
years. Consumption drops by $1.8 \%$ after last year's drought and then slightly decreases on average $0.74 \%$ in the following year.

The regression estimates show mixed relationships between drought and expenditures for non-food goods and services; there is a $1.4 \%$ increase immediately after a drought occurred in that year. The increase on total consumption is probably contributed mostly by the expenditures for less frequent non-food as we observe a 3.5\% mean increase of this expenditure during the year of drought occurred. On the other hand, the results in Table 9 show a significant correlation between last year drought and frequently purchased non-food, suggesting that a drought indeed has adverse effects on non-food consumption, at least after a year it occurred. The estimated effect is a $5 \%$ drop in the expenditures for household's goods and services that are consumed on a regular basis.

Last but not least, Table 9 shows interesting results with regards to positive links between drought at IFLS year and expenditures. There may be several possible reasons leading to household monthly spending increasing directly after a drought occurs, including a possibility that it was a necessary reaction to the shock. For example, people have to buy clean water during a drought period when clean water is scarce (for drinking/food or sometimes also for secondary/ another purpose like for taking a bath, for farm/animal husbandry). A rise in spending for medical costs (included in less frequent consumption) may also happen during extreme weather as drought is associated with an increased risk of some diseases (Lohmann \& Lechtenfeld, 2015).

\subsubsection{The effect of droughts on the outcomes of different types of households}

Next, we look at the impact of droughts on different household groups. We classify households into 5 socio-economic status groups (5 quantiles), based on their monthly total expenditures. Recall that in the previous regression models, variable monthly expenditure is used as one of the controls and termed as "economic condition" variable (see Table 8 and 9). To quantify the effect of each economic group, we use only observations of the related socio-economic status. For brevity, we only report the results of 3 groups: "poor" (lowest quantile, below 20\%), "average" (3rd quantile, between $40^{\text {th }}-$ $60 \%$ ) and "rich" (the $5^{\text {th }}$ or the highest quantile). Table 10 a to $10 \mathrm{c}$ report the estimated effects for incomes and Table 11a to 11c are the summary for the expenditures of each group. 


\subsubsection{Drought impacts on the income of the poor, average and rich households}

A detailed summary in Table 10a reports the results of estimated effects of drought on the poor household group. For poor households, we do not find convincing evidence that drought episodes have impacts on incomes. There is only one significant result that shows a drought episode is associated with income (significance at $90 \%$ confidence level), which is a positive impact.

Table 10a Impact of droughts on the incomes of poor households

\begin{tabular}{|c|c|c|c|c|c|}
\hline & \multicolumn{5}{|c|}{ DEPENDENT VARIABLE: HOUSEHOLD INCOMES (log) } \\
\hline & $\begin{array}{c}\text { Total } \\
\text { Income }\end{array}$ & $\begin{array}{l}\text { Farm } \\
\text { Income }\end{array}$ & $\begin{array}{l}\text { Non-Farm } \\
\text { Income }\end{array}$ & $\begin{array}{c}\text { Selling } \\
\text { Farm Assets }\end{array}$ & $\begin{array}{l}\text { Selling All } \\
\text { Type of } \\
\text { Assets }\end{array}$ \\
\hline Drought $t_{0}(1 / 0)$ & $\begin{array}{l}-0.0137 \\
(0.0728)\end{array}$ & $\begin{array}{c}0.0513 \\
(0.0997)\end{array}$ & $\begin{array}{c}0.0014 \\
(0.1425)\end{array}$ & $\begin{array}{c}0.7062 \\
(0.5434)\end{array}$ & $\begin{array}{l}-0.2838 \\
(0.2770)\end{array}$ \\
\hline Drought $t_{1}(1 / 0)$ & $\begin{array}{c}0.0450 \\
(0.1930)\end{array}$ & $\begin{array}{c}0.3275^{*} \\
(0.1860)\end{array}$ & $\begin{array}{l}-0.2995 \\
(0.4607)\end{array}$ & $\begin{array}{c}-0.4591 \\
(1.1128)\end{array}$ & $\begin{array}{l}-0.0080 \\
(0.9178)\end{array}$ \\
\hline Drought $t_{2}(1 / 0)$ & $\begin{array}{c}-0.0098 \\
(0.0805)\end{array}$ & $\begin{array}{c}0.0412 \\
(0.1090)\end{array}$ & $\begin{array}{c}0.0774 \\
(0.1667)\end{array}$ & $\begin{array}{c}0.5869 \\
(0.6941)\end{array}$ & $\begin{array}{c}0.3178 \\
(0.2699)\end{array}$ \\
\hline Drought $t_{3}(1 / 0)$ & $\begin{array}{l}-0.1167 \\
(0.0836)\end{array}$ & $\begin{array}{l}-0.0727 \\
(0.1071)\end{array}$ & $\begin{array}{l}-0.0625 \\
(0.1824)\end{array}$ & $\begin{array}{l}-0.7638 \\
(0.7564)\end{array}$ & $\begin{array}{c}-0.2372 \\
(0.3429)\end{array}$ \\
\hline Household size & $\begin{array}{c}-0.0176^{* * *} \\
(0.0030)\end{array}$ & $\begin{array}{l}-0.0248^{* * *} \\
(0.0028)\end{array}$ & $\begin{array}{c}-0.0588^{* * *} \\
(0.0026)\end{array}$ & $\begin{array}{c}0.0280 \\
(0.0175)\end{array}$ & $\begin{array}{c}0.0003 \\
(0.0048)\end{array}$ \\
\hline Move out & $\begin{array}{l}0.1291^{* *} \\
(0.0449)\end{array}$ & $\begin{array}{c}0.0906 \\
(0.0540)\end{array}$ & $\begin{array}{l}-0.0749 \\
(0.0537)\end{array}$ & $\begin{array}{c}-0.1804 \\
(0.0969)\end{array}$ & $\begin{array}{c}0.0689 \\
(0.0420)\end{array}$ \\
\hline Own a farmland & $\begin{array}{l}0.1890^{* * *} \\
(0.0283)\end{array}$ & $\begin{array}{c}-0.1131 \\
(0.1274)\end{array}$ & $\begin{array}{c}-0.0291 \\
(0.0776)\end{array}$ & $\begin{array}{c}0.6369^{* *} \\
(0.2249)\end{array}$ & $\begin{array}{c}-0.4790^{* * *} \\
(0.0523)\end{array}$ \\
\hline Run a farm & $\begin{array}{l}0.1620^{* *} \\
(0.0393)\end{array}$ & $\begin{array}{c}0.4115^{* *} \\
(0.1023)\end{array}$ & $\begin{array}{c}0.1451^{*} \\
(0.0599)\end{array}$ & $\begin{array}{c}0.2571 \\
(0.3121)\end{array}$ & $\begin{array}{c}0.3630^{*} \\
(0.1363)\end{array}$ \\
\hline Total assets $>100 \mathrm{~m}$ & $\begin{array}{l}0.2360^{* * *} \\
(0.0437)\end{array}$ & $\begin{array}{c}0.1731^{*} \\
(0.0645)\end{array}$ & $\begin{array}{l}0.4687^{* * *} \\
(0.0803)\end{array}$ & $\begin{array}{l}-0.0218 \\
(0.3478)\end{array}$ & $\begin{array}{c}0.2549^{*} \\
(0.1090)\end{array}$ \\
\hline Receive wage & $\begin{array}{l}1.1494^{* * *} \\
(0.0484)\end{array}$ & $\begin{array}{l}0.5856^{* * *} \\
(0.0232)\end{array}$ & $\begin{array}{l}0.7320^{* * *} \\
(0.0248)\end{array}$ & $\begin{array}{c}-0.1441 \\
(0.1616)\end{array}$ & $\begin{array}{l}0.2704^{* * *} \\
(0.0382)\end{array}$ \\
\hline Age of head & $\begin{array}{c}0.0007 \\
(0.0009)\end{array}$ & $\begin{array}{l}0.0039^{* * *} \\
(0.0006)\end{array}$ & $\begin{array}{c}0.0002 \\
(0.0023)\end{array}$ & $\begin{array}{c}0.0190 \\
(0.0172)\end{array}$ & $\begin{array}{l}-0.0052 \\
(0.0070)\end{array}$ \\
\hline Head's gender & $\begin{array}{l}-0.0126 \\
(0.0314)\end{array}$ & $\begin{array}{l}0.1452^{* * *} \\
(0.0196)\end{array}$ & $\begin{array}{l}-0.1663^{*} \\
(0.0621)\end{array}$ & $\begin{array}{l}-1.3818^{* *} \\
(0.3198)\end{array}$ & $\begin{array}{c}-0.9292^{* * *} \\
(0.0643)\end{array}$ \\
\hline Educ (Uni) & $\begin{array}{l}-0.1285^{*} \\
(0.0533)\end{array}$ & $\begin{array}{l}-0.2370 \\
(0.1210)\end{array}$ & $\begin{array}{c}0.1489 \\
(0.1172)\end{array}$ & $\begin{array}{c}0.7132 \\
(0.7971)\end{array}$ & $\begin{array}{l}-0.2168 \\
(0.2387)\end{array}$ \\
\hline Educ (High) & $\begin{array}{c}0.0069 \\
(0.0615)\end{array}$ & $\begin{array}{c}0.0394 \\
(0.0946)\end{array}$ & $\begin{array}{l}-0.1964^{*} \\
(0.0730)\end{array}$ & $\begin{array}{l}0.3272^{* *} \\
(0.0823)\end{array}$ & $\begin{array}{l}-0.4136^{*} \\
(0.1512)\end{array}$ \\
\hline Educ (Junior) & $\begin{array}{l}-0.0850 \\
(0.1074)\end{array}$ & $\begin{array}{c}-0.0162 \\
(0.0962)\end{array}$ & $\begin{array}{l}-0.0827 \\
(0.0765)\end{array}$ & $\begin{array}{c}-0.4492 \\
(0.5665)\end{array}$ & $\begin{array}{l}-0.2506 \\
(0.2579)\end{array}$ \\
\hline Educ (Elemen) & $\begin{array}{c}0.1169 \\
(0.0831)\end{array}$ & $\begin{array}{c}-0.1001 \\
(0.0565)\end{array}$ & $\begin{array}{c}0.0547 \\
(0.0947)\end{array}$ & $\begin{array}{c}-0.4451 \\
(0.2944)\end{array}$ & $\begin{array}{c}0.0634 \\
(0.0403)\end{array}$ \\
\hline Can read and write & $\begin{array}{c}0.0503 \\
(0.0628)\end{array}$ & $\begin{array}{c}0.0568 \\
(0.0675)\end{array}$ & $\begin{array}{c}0.2595 \\
(0.1333)\end{array}$ & $\begin{array}{l}1.0199^{* *} \\
(0.3335)\end{array}$ & $\begin{array}{c}0.2856 \\
(0.1900)\end{array}$ \\
\hline$N$ & 4,877 & 3,109 & 2,457 & 729 & 1,769 \\
\hline Adj. R2 & 0.5961 & 0.4647 & 0.3209 & 0.2025 & 0.2333 \\
\hline
\end{tabular}


Note: Driscoll and Kraay standard errors (in parentheses) are robust to heteroscedasticity and potential bias (due to error's spatial and temporal dependences). ${ }^{*} p<0.10,{ }^{* *} p<0.05,{ }^{* * *} p<0.01$ denote statistical significance for the relationship. Drought at IFLS year refers to droughts that occurred in 1993, 1997, 2000, 2007 and 2014. Drought (t-1) refers to droughts that occurred in 1992, 1996, 1999, 2006 and 2013. Drought (t-2) refers to droughts that occurred in 1991, 1995, 1998, 2005 and 2012. Drought (t-3) refers to droughts that occurred in 1990, 1994, 1997, 2004 and 2011. Models are panel household and time fixed-effects. Poor is defined as a socio-economic condition when household monthly expenditure falls in the lowest quantile (the $1^{\text {st }}$ ).

The results in average households (Table 10b) show more evidence of drought-incomes nexus than in poor household's models. There are significant correlations between drought events and reductions in all type of incomes. The relationship's sign, however, changes in 3 years after the drought occurred (compare row Drought $t_{3}$ and its previous rows). The scale and significance of the impact depend on several factors, most notably are attributed to drought's timing. For example, a drought this year is associated with decreased changes in incomes from non-farm business and selling assets of average households. While a drought last year affects negatively incomes from farm and nonfarm, which in the case for non-farm the estimated effect is higher than the one caused by a drought this year. Overall, for average households, income affected most should there be drought is non-farm income. The predicted effect appeared to be last until 2 years after the drought occurred (see column Non-Farm Income). The negative impact seems to fade away in the $3^{\text {rd }}$ year after the drought occurred and becomes positive (despite less significant).

Table 10b Impact of droughts on the incomes of average households

\begin{tabular}{lccccc}
\hline & \multicolumn{3}{c}{ DEPENDENT VARIABLE: HOUSEHOLD INCOMES (log) } \\
\cline { 2 - 6 } & $\begin{array}{c}\text { Total } \\
\text { Income }\end{array}$ & $\begin{array}{c}\text { Farm } \\
\text { Income }\end{array}$ & $\begin{array}{c}\text { Non-Farm } \\
\text { Income }\end{array}$ & $\begin{array}{c}\text { Selling } \\
\text { Farm Assets }\end{array}$ & $\begin{array}{c}\text { Selling All } \\
\text { Type of } \\
\text { Assets }\end{array}$ \\
\hline Drought $t_{0}(1 / 0)$ & 0.0800 & -0.0365 & $-0.2820^{* * *}$ & $-0.6709^{* *}$ & $-0.5049^{* * *}$ \\
& $(0.0924)$ & $(0.0254)$ & $(0.0537)$ & $(0.2005)$ & $(0.0766)$ \\
Drought $t_{1}(1 / 0)$ & -0.0296 & $-0.5090^{* *}$ & $-0.5100^{* * *}$ & -1.5392 & -0.1419 \\
& $(0.0277)$ & $(0.1382)$ & $(0.0989)$ & $(0.8653)$ & $(0.1689)$ \\
Drought $t_{2}(1 / 0)$ & $-0.0965^{* *}$ & -0.0000 & $-0.1793^{* *}$ & 0.3680 & 0.0442 \\
& $(0.0286)$ & $(0.0479)$ & $(0.0489)$ & $(0.2826)$ & $(0.0496)$ \\
Drought $t_{3}(1 / 0)$ & 0.0310 & $0.1895^{* * *}$ & $0.2055^{*}$ & 0.1458 & -0.0037 \\
& $(0.0370)$ & $(0.0341)$ & $(0.0906)$ & $(0.2908)$ & $(0.1923)$ \\
Household size & $-0.0565^{* * *}$ & $-0.0452^{* * *}$ & $-0.0783^{* * *}$ & $-0.2019^{* * *}$ & $-0.0426^{* *}$ \\
& $(0.0072)$ & $(0.0075)$ & $(0.0069)$ & $(0.0278)$ & $(0.0139)$ \\
Move out & $-0.0591^{* *}$ & -0.1047 & 0.0680 & $1.5511^{* *}$ & 0.2106 \\
& $(0.0156)$ & $(0.0718)$ & $(0.0445)$ & $(0.3479)$ & $(0.1202)$ \\
Own a farmland & $0.3333^{* * *}$ & $0.2890^{*}$ & $0.2544^{*}$ & -0.4733 & $-0.8185^{* *}$ \\
& $(0.0361)$ & $(0.1091)$ & $(0.1121)$ & $(0.2298)$ & $(0.2484)$ \\
Run a farm & $0.1879^{* * *}$ & $0.3163^{* * *}$ & $-0.3979^{* *}$ & $1.3064^{* * *}$ & $0.5562^{*}$ \\
& $(0.0402)$ & $(0.0508)$ & $(0.1037)$ & $(0.2735)$ & $(0.2186)$ \\
Rich Assets & $0.3214^{* * *}$ & $0.4483^{* * *}$ & 0.1362 & $0.9429^{* * *}$ & $0.7635^{* * *}$ \\
& $(0.0207)$ & $(0.0727)$ & $(0.0668)$ & $(0.1223)$ & $(0.1319)$ \\
Receive wage & $1.1729^{* * *}$ & $0.3541^{* *}$ & $0.7322^{* * *}$ & -0.4322 & -0.1486
\end{tabular}




\begin{tabular}{lccccc} 
& $(0.1089)$ & $(0.1060)$ & $(0.1057)$ & $(0.3635)$ & $(0.2088)$ \\
Age of head & $0.0077^{* * *}$ & 0.0040 & $0.0109^{*}$ & $0.0420^{* * *}$ & $0.0343^{* * *}$ \\
& $(0.0016)$ & $(0.0019)$ & $(0.0043)$ & $(0.0058)$ & $(0.0014)$ \\
Head's gender & 0.0491 & -0.0363 & -0.0610 & $2.6019^{* *}$ & $0.3370^{* *}$ \\
& $(0.0482)$ & $(0.0616)$ & $(0.0989)$ & $(0.9107)$ & $(0.0761)$ \\
Educ (Uni) & $0.2759^{* *}$ & -0.1494 & 0.0427 & $-1.3708^{* *}$ & -0.1190 \\
& $(0.0946)$ & $(0.1787)$ & $(0.0563)$ & $(0.4025)$ & $(0.1719)$ \\
Educ (High) & $0.2307^{*}$ & 0.2471 & 0.0090 & $2.1054^{*}$ & $1.0842^{* * *}$ \\
& $(0.0926)$ & $(0.1294)$ & $(0.0962)$ & $(0.9674)$ & $(0.1490)$ \\
Educ (Junior) & $-0.1914^{* *}$ & $-0.3116^{* * *}$ & 0.0609 & $-1.9795^{* *}$ & $-0.9388^{* * *}$ \\
& $(0.0656)$ & $(0.0592)$ & $(0.1046)$ & $(0.5718)$ & $(0.1389)$ \\
Educ (Elemen) & 0.1278 & 0.0368 & 0.0862 & $-0.5885^{* *}$ & -0.0464 \\
& $(0.0817)$ & $(0.0366)$ & $(0.0512)$ & $(0.1858)$ & $(0.1121)$ \\
Can read and write & $0.1193^{* *}$ & $0.2008^{* * *}$ & 0.2328 & $1.2707^{* * *}$ & $0.8677^{* *}$ \\
& $(0.0312)$ & $(0.0306)$ & $(0.1172)$ & $(0.1669)$ & $(0.2133)$ \\
\hline$N$ & 4,712 & 2,453 & 2,105 & 492 & 1,449 \\
Adj. R2 & 0.5010 & 0.3771 & 0.3252 & 0.6802 & 0.2621 \\
\hline
\end{tabular}

Note: Driscoll and Kraay standard errors (in parentheses) are robust to heteroscedasticity and potential bias (due to error's spatial and temporal dependences). ${ }^{*} p<0.10,{ }^{* *} p<0.05, " * * 0<0.01$ denote statistical significance for the relationship. Drought at IFLS year refers to droughts that occurred in 1993, 1997, 2000, 2007 and 2014. Drought (t-1) refers to droughts that occurred in 1992, 1996, 1999, 2006 and 2013. Drought (t-2) refers to droughts that occurred in 1991, 1995, 1998, 2005 and 2012. Drought (t-3) refers to droughts that occurred in 1990, 1994, 1997, 2004 and 2011. Models are panel household and time fixed-effects. Average is defined as a socio-economic condition when household monthly expenditure falls in the midst quantile (the $3^{\text {rd }}$ ).

For rich households, there are mixed results with respect to income's type and drought's timing (Table 10c). The effect of drought is significantly negative in 3 years after it occurs (on all incomes but selling all assets), but before that, a drought is associated with positive changes in incomes (see row Drought t0, Drought t1, and Drought t2). Specifically, a drought occurred 3 years ago predicts a 28\% 23\%, and 32\% drop in total income, farm income, and non-farm income, respectively. On the other hand, a contemporaneous drought (Drought $t_{0}$ ) is significantly associated with a $9 \%$ increase in total income. A robust link between drought and variations in income from selling all type of assets is however obvious in the last two years after it occurs. Selling assets is likely a more preferred coping strategy for the rich than non-rich household groups (having compared the results regarding selling assets impacts in Table 10a, Table 10b and Table 10c).

Table 10c Impact of droughts on the incomes of rich households

\begin{tabular}{lccccc}
\hline & \multicolumn{4}{c}{ DEPENDENT VARIABLE: HOUSEHOLD INCOMES (log) } \\
\cline { 2 - 6 } & $\begin{array}{c}\text { Total } \\
\text { Income }\end{array}$ & $\begin{array}{c}\text { Farm } \\
\text { Income }\end{array}$ & $\begin{array}{c}\text { Non-Farm } \\
\text { Income }\end{array}$ & $\begin{array}{c}\text { Selling } \\
\text { Farm Assets }\end{array}$ & $\begin{array}{c}\text { Selling all } \\
\text { type assets }\end{array}$ \\
\hline Drought $t_{0}(1 / 0)$ & $0.0912^{*}$ & -0.0129 & 0.0864 & -0.2408 & $0.4674^{* *}$ \\
& $(0.0399)$ & $(0.0529)$ & $(0.1560)$ & $(0.5398)$ & $(0.1420)$ \\
Drought $t_{1}(1 / 0)$ & 0.0374 & 0.0091 & $0.5872^{* * *}$ & -0.2896 & $0.8057^{* *}$ \\
& $(0.1065)$ & $(0.1520)$ & $(0.0369)$ & $(0.4987)$ & $(0.1784)$ \\
Drought $t_{2}(1 / 0)$ & 0.0371 & $0.2001^{* *}$ & 0.1494 & -1.0331 & $0.5518^{* *}$ \\
& $(0.0442)$ & $(0.0709)$ & $(0.0896)$ & $(0.8033)$ & $(0.1397)$ \\
Drought $t_{3}(1 / 0)$ & $-0.2822^{* * *}$ & $-0.2280^{* *}$ & $-0.3247^{* * *}$ & $-5.6870^{* * *}$ & -0.1284
\end{tabular}




\begin{tabular}{|c|c|c|c|c|c|}
\hline \multirow{3}{*}{ Household size } & $(0.0343)$ & $(0.0633)$ & $(0.0345)$ & $(0.6501)$ & $(0.1771)$ \\
\hline & $-0.1164 * * *$ & $-0.1746^{* * *}$ & $-0.1366^{* * *}$ & $0.2954^{* *}$ & $-0.2041^{* * *}$ \\
\hline & $(0.0191)$ & $(0.0217)$ & $(0.0233)$ & $(0.0879)$ & $(0.0136)$ \\
\hline \multirow[t]{2}{*}{ Move out } & 0.0394 & $0.2364^{* *}$ & 0.0586 & -0.1704 & 0.3053 \\
\hline & $(0.0905)$ & $(0.0765)$ & $(0.1208)$ & $(0.1650)$ & $(0.2514)$ \\
\hline \multirow[t]{2}{*}{ Own a farmland } & $-0.0890 *$ & $-0.6696^{* * *}$ & $0.0970^{*}$ & 0.5923 & $0.5671^{* *}$ \\
\hline & $(0.0377)$ & $(0.1079)$ & $(0.0447)$ & $(0.4654)$ & $(0.1565)$ \\
\hline \multirow[t]{2}{*}{ Run a farm } & $0.3821^{* * *}$ & $0.4823^{* * *}$ & -0.0543 & $2.6602^{* *}$ & 0.2784 \\
\hline & $(0.0630)$ & $(0.0849)$ & $(0.0711)$ & $(0.8215)$ & $(0.2113)$ \\
\hline \multirow[t]{2}{*}{ Rich Assets } & $0.5334^{* * *}$ & $0.2961^{* * *}$ & $0.4498^{* *}$ & 0.5869 & 0.0676 \\
\hline & $(0.0545)$ & $(0.0427)$ & $(0.0996)$ & $(0.2872)$ & $(0.1471)$ \\
\hline \multirow[t]{2}{*}{ Receive wage } & $1.1556^{* * *}$ & $0.2845^{* *}$ & $0.9364^{* * *}$ & 0.2615 & 0.3274 \\
\hline & $(0.0905)$ & $(0.0796)$ & $(0.0657)$ & $(0.2012)$ & $(0.1633)$ \\
\hline \multirow[t]{2}{*}{ Age of head } & $0.0034^{*}$ & $-0.0071^{*}$ & 0.0001 & $-0.0657^{* * *}$ & $-0.0330^{* *}$ \\
\hline & $(0.0015)$ & $(0.0031)$ & $(0.0020)$ & $(0.0052)$ & $(0.0088)$ \\
\hline \multirow[t]{2}{*}{ Head's gender } & -0.0523 & -0.1370 & -0.2184 & -0.3882 & $1.0223^{*}$ \\
\hline & $(0.0658)$ & $(0.1145)$ & $(0.1757)$ & $(0.3551)$ & $(0.4124)$ \\
\hline \multirow[t]{2}{*}{ Educ (Uni) } & -0.1546 & -0.0712 & -0.0999 & -0.0352 & -0.5711 \\
\hline & $(0.1038)$ & $(0.0729)$ & $(0.0708)$ & $(0.3233)$ & $(0.3857)$ \\
\hline \multirow[t]{2}{*}{ Educ (High) } & 0.0667 & -0.0714 & $-0.3564^{*}$ & $-1.8814^{* * *}$ & $1.1353^{* *}$ \\
\hline & $(0.0572)$ & $(0.1650)$ & $(0.1530)$ & $(0.2613)$ & $(0.4038)$ \\
\hline \multirow{2}{*}{ Educ (Junior) } & 0.2575 & $0.3855^{* *}$ & 0.0688 & $5.7832^{* * *}$ & 0.1575 \\
\hline & $(0.1467)$ & $(0.1176)$ & $(0.3437)$ & $(0.6908)$ & $(0.1232)$ \\
\hline \multirow[t]{2}{*}{ Educ (Elemen) } & -0.1683 & $-0.3669^{* * *}$ & $0.4974^{* *}$ & $-6.1518^{* * *}$ & $-1.5051^{* * *}$ \\
\hline & $(0.1190)$ & $(0.0578)$ & $(0.1423)$ & $(0.9630)$ & $(0.1103)$ \\
\hline \multirow[t]{2}{*}{ Can read and write } & 0.0746 & $-0.2351^{* *}$ & $-0.7890^{* *}$ & $0.9269^{*}$ & 0.3823 \\
\hline & $(0.0482)$ & $(0.0774)$ & $(0.1747)$ & $(0.3952)$ & $(0.5476)$ \\
\hline$N$ & 4,620 & 2,003 & 2,101 & 407 & 1,327 \\
\hline Adj. R2 & 0.5007 & 0.3751 & 0.4436 & 0.6274 & 0.2004 \\
\hline
\end{tabular}

Note: Driscoll and Kraay standard errors (in parentheses) are robust to heteroscedasticity and potential bias (due to error's spatial and temporal dependences). ${ }^{*} p<0.10,{ }^{* *} p<0.05,{ }^{* * *} p<0.01$ denote statistical significance for the relationship. Drought at IFLS year refers to droughts that occurred in 1993, 1997, 2000, 2007 and 2014. Drought (t-1) refers to droughts that occurred in 1992, 1996, 1999, 2006 and 2013. Drought (t-2) refers to droughts that occurred in 1991, 1995, 1998, 2005 and 2012. Drought (t-3) refers to droughts that occurred in 1990,1994,1997, 2004 and 2011. Models are panel household and time fixed-effects Rich is defined as a socio-economic condition when household monthly expenditure falls in the highest quantile (the $5^{\text {th }}$ ).

\subsubsection{Drought impact on the monthly expenditure of the poor, average and rich households}

The impacts of drought on expenditure evidently vary between one economic group and another. The results also indicate heterogeneous effects with respect to expenditure types and drought's timing. For poor households (Table 11a), what seems to be apparent is a positive effect of a contemporaneous drought (significant in monthly total and nonfood expenditures). A year after the drought occurred, then there are significant reductions in spending on food, education and non-food, from as little as $2 \%$ to $11 \%$. While drought seems to negatively affect almost all type of expenses of poor (at different drought's timing), there is no significant result indicating that it has an adverse effect on total monthly expenditure. 
Table 11a Impact of droughts on the monthly expenditure of poor households

\begin{tabular}{|c|c|c|c|c|c|c|}
\hline & \multicolumn{6}{|c|}{ DEPENDENT VARIABLE HOUSEHOLD EXPENDITURES $(\log )$} \\
\hline & $\begin{array}{c}\text { Total } \\
\text { Expenditure }\end{array}$ & $\begin{array}{c}\text { Food } \\
\text { Consumption }\end{array}$ & $\begin{array}{c}\text { Non Food } \\
\text { Consumption }\end{array}$ & $\begin{array}{l}\text { Education } \\
\text { Expenses }\end{array}$ & $\begin{array}{l}\text { Non-Food } \\
\text { Frequent }\end{array}$ & $\begin{array}{l}\text { Non-Food } \\
\text { Less }\end{array}$ \\
\hline Drought $t_{0}(1 / 0)$ & $\begin{array}{c}0.0219^{*} \\
(0.0092)\end{array}$ & $\begin{array}{c}0.0111 \\
(0.0096)\end{array}$ & $\begin{array}{l}0.0642^{* * *} \\
(0.0109)\end{array}$ & $\begin{array}{c}0.0764 \\
(0.0401)\end{array}$ & $\begin{array}{c}0.0417 \\
(0.0332)\end{array}$ & $\begin{array}{c}0.0711^{*} \\
(0.0283)\end{array}$ \\
\hline Drought $t_{1}(1 / 0)$ & $\begin{array}{c}0.0095 \\
(0.0082)\end{array}$ & $\begin{array}{l}0.0572^{* * *} \\
(0.0110)\end{array}$ & $\begin{array}{l}-0.0446^{* *} \\
(0.0122)\end{array}$ & $\begin{array}{c}-0.1043 \\
(0.0495)\end{array}$ & $\begin{array}{c}-0.1105^{* * *} \\
(0.0053)\end{array}$ & $\begin{array}{c}-0.0383 \\
(0.0299)\end{array}$ \\
\hline Drought $t_{2}(1 / 0)$ & $\begin{array}{l}-0.0019 \\
(0.0052)\end{array}$ & $\begin{array}{l}-0.0204^{*} \\
(0.0092)\end{array}$ & $\begin{array}{c}0.0023 \\
(0.0082)\end{array}$ & $\begin{array}{c}-0.0442 \\
(0.0623)\end{array}$ & $\begin{array}{c}0.0056 \\
(0.0163)\end{array}$ & $\begin{array}{c}-0.0325 \\
(0.0305)\end{array}$ \\
\hline Drought $t_{3}(1 / 0)$ & $\begin{array}{c}-0.0198 \\
(0.0221)\end{array}$ & $\begin{array}{c}-0.0157 \\
(0.0168)\end{array}$ & $\begin{array}{l}-0.0337 \\
(0.0210)\end{array}$ & $\begin{array}{l}-0.1094^{*} \\
(0.0494)\end{array}$ & $\begin{array}{c}-0.0458 \\
(0.0294)\end{array}$ & $\begin{array}{l}-0.1120^{*} \\
(0.0497)\end{array}$ \\
\hline Household size & $\begin{array}{c}-0.0361^{* * *} \\
(0.0018)\end{array}$ & $\begin{array}{c}-0.0417^{* * *} \\
(0.0024)\end{array}$ & $\begin{array}{c}-0.0313^{* * *} \\
(0.0013)\end{array}$ & $\begin{array}{c}-0.0054 \\
(0.0044)\end{array}$ & $\begin{array}{c}-0.0257^{* * *} \\
(0.0022)\end{array}$ & $\begin{array}{c}-0.0285^{* * *} \\
(0.0035)\end{array}$ \\
\hline Move out & $\begin{array}{l}-0.0198^{*} \\
(0.0076)\end{array}$ & $\begin{array}{c}-0.0177 \\
(0.0162)\end{array}$ & $\begin{array}{c}-0.0571^{* *} \\
(0.0175)\end{array}$ & $\begin{array}{c}-0.0733 \\
(0.0504)\end{array}$ & $\begin{array}{c}-0.0052 \\
(0.0147)\end{array}$ & $\begin{array}{l}-0.1171^{* *} \\
(0.0310)\end{array}$ \\
\hline Own a farmland & $\begin{array}{c}0.0080 \\
(0.0129)\end{array}$ & $\begin{array}{c}-0.0095 \\
(0.0085)\end{array}$ & $\begin{array}{c}0.0382 \\
(0.0269)\end{array}$ & $\begin{array}{c}0.0502 \\
(0.0347)\end{array}$ & $\begin{array}{c}0.0633 \\
(0.0376)\end{array}$ & $\begin{array}{c}0.0463 \\
(0.0812)\end{array}$ \\
\hline Run a farm & $\begin{array}{c}-0.0270 \\
(0.0196)\end{array}$ & $\begin{array}{c}-0.0044 \\
(0.0210)\end{array}$ & $\begin{array}{l}-0.0736^{* *} \\
(0.0161)\end{array}$ & $\begin{array}{c}-0.0013 \\
(0.0967)\end{array}$ & $\begin{array}{l}-0.0734^{*} \\
(0.0326)\end{array}$ & $\begin{array}{c}-0.0958^{* *} \\
(0.0283)\end{array}$ \\
\hline Rich Assets & $\begin{array}{c}0.0296 \\
(0.0140)\end{array}$ & $\begin{array}{c}0.0381^{*} \\
(0.0141)\end{array}$ & $\begin{array}{l}0.0940^{* *} \\
(0.0217)\end{array}$ & $\begin{array}{c}0.0100 \\
(0.0386)\end{array}$ & $\begin{array}{c}0.0333 \\
(0.0303)\end{array}$ & $\begin{array}{c}0.0447 \\
(0.0346)\end{array}$ \\
\hline Receive wage & $\begin{array}{c}0.0127 \\
(0.0175)\end{array}$ & $\begin{array}{c}0.0457^{* *} \\
(0.0131)\end{array}$ & $\begin{array}{c}-0.0544^{* *} \\
(0.0133)\end{array}$ & $\begin{array}{c}0.0134 \\
(0.0397)\end{array}$ & $\begin{array}{c}0.0114 \\
(0.0134)\end{array}$ & $\begin{array}{c}-0.0816^{* * *} \\
(0.0165)\end{array}$ \\
\hline Age of head & $\begin{array}{l}-0.0008^{* *} \\
(0.0002)\end{array}$ & $\begin{array}{c}0.0007 \\
(0.0005)\end{array}$ & $\begin{array}{l}-0.0001 \\
(0.0005)\end{array}$ & $\begin{array}{l}-0.0083^{*} \\
(0.0032)\end{array}$ & $\begin{array}{c}0.0012 \\
(0.0010)\end{array}$ & $\begin{array}{c}0.0024 \\
(0.0014)\end{array}$ \\
\hline Head's gender & $\begin{array}{l}0.0418^{* * *} \\
(0.0057)\end{array}$ & $\begin{array}{c}0.0537^{* *} \\
(0.0185)\end{array}$ & $\begin{array}{c}0.0372^{* *} \\
(0.0104)\end{array}$ & $\begin{array}{c}-0.0764 \\
(0.0582)\end{array}$ & $\begin{array}{c}0.0075 \\
(0.0227)\end{array}$ & $\begin{array}{c}0.0140 \\
(0.0189)\end{array}$ \\
\hline Educ & $\begin{array}{c}0.0247 \\
(0.0175)\end{array}$ & $\begin{array}{c}0.0097 \\
(0.0360)\end{array}$ & $\begin{array}{c}0.0175 \\
(0.0160)\end{array}$ & $\begin{array}{c}0.1365^{*} \\
(0.0548)\end{array}$ & $\begin{array}{c}0.0358 \\
(0.0310)\end{array}$ & $\begin{array}{l}0.1472^{* *} \\
(0.0365)\end{array}$ \\
\hline Educ (High) & $\begin{array}{c}-0.0252 \\
(0.0203)\end{array}$ & $\begin{array}{c}-0.0212 \\
(0.0155)\end{array}$ & $\begin{array}{c}-0.0306 \\
(0.0271)\end{array}$ & $\begin{array}{l}-0.1366^{*} \\
(0.0546)\end{array}$ & $\begin{array}{l}0.1196^{* *} \\
(0.0326)\end{array}$ & $\begin{array}{c}0.0129 \\
(0.0636)\end{array}$ \\
\hline Educ (Junior) & $\begin{array}{c}0.0679^{* *} \\
(0.0150)\end{array}$ & $\begin{array}{l}0.0595^{* *} \\
(0.0177)\end{array}$ & $\begin{array}{c}0.0501^{*} \\
(0.0185)\end{array}$ & $\begin{array}{l}0.2865^{* *} \\
(0.0649)\end{array}$ & $\begin{array}{l}-0.0977^{*} \\
(0.0435)\end{array}$ & $\begin{array}{l}-0.1256^{* *} \\
(0.0393)\end{array}$ \\
\hline Educ (Elemen) & $\begin{array}{l}-0.0442^{* *} \\
(0.0133)\end{array}$ & $\begin{array}{c}-0.0353^{* *} \\
(0.0084)\end{array}$ & $\begin{array}{c}-0.0460 \\
(0.0289)\end{array}$ & $\begin{array}{l}-0.1909^{* *} \\
(0.0590)\end{array}$ & $\begin{array}{c}0.0523 \\
(0.0394)\end{array}$ & $\begin{array}{c}0.0019 \\
(0.0190)\end{array}$ \\
\hline Can read and write & $\begin{array}{c}-0.0333 \\
(0.0174)\end{array}$ & $\begin{array}{c}-0.0395^{* * *} \\
(0.0081)\end{array}$ & $\begin{array}{l}-0.0048 \\
(0.0318)\end{array}$ & $\begin{array}{c}0.0222 \\
(0.0538)\end{array}$ & $\begin{array}{c}-0.1267^{* * *} \\
(0.0203)\end{array}$ & $\begin{array}{c}-0.0800 \\
(0.0672)\end{array}$ \\
\hline$N$ & 5,577 & 5,495 & 5,514 & 3,349 & 5,486 & 5,495 \\
\hline $\mathrm{Ad}$ & 0.1160 & 0.1149 & 0.0694 & 0.1143 & 0.1282 & 0.1128 \\
\hline
\end{tabular}

Note: Driscoll and Kraay standard errors (in parentheses) are robust to heteroscedasticity and potential bias (due to error's spatial and temporal dependences). ${ }^{*} p<0.10,{ }^{* *} p<0.05,{ }^{* * *} p<0.01$ denote statistical significance for the relationship. Drought at IFLS year refers to droughts that occurred in 1993, 1997, 2000, 2007 and 2014. Drought (t-1) refers to droughts that occurred in 1992, 1996, 1999, 2006 and 2013. Drought (t-2) refers to droughts that occurred in 1991, 1995, 1998, 2005 and 2012. Drought (t-3) refers to droughts that occurred in 1990,1994, 1997, 2004 and 2011. Models are panel household and time fixed-effects with control variables such as household's time-varying characteristics: size, whether own farmland, farm value $>10 \mathrm{~m}$, receive wages, total assets $>100 \mathrm{~m}$, and head's profile (age, gender, education). Rich is defined as a socio-economic condition when household monthly expenditure falls in the highest quantile (the $5^{\text {th }}$ ).

There are interesting results for average households in Table 11b, for example, robust short-term effects on education expenditure. A contemporaneous drought is associated with a $17.5 \%$ drop in spending on education while the last year drought would predict a decrease by $13.6 \%$. On the other hand, it appears that average households do not 
experience any drought effect on non-food expenditure. However, when the non-food expense is broken down into frequently and less frequently purchased goods/services, there are positive relationships between drought episodes and the non-food outcomes. We find impacts on food and total expenditure show a changing pattern.

Table 11b Impact of droughts on the monthly expenditure of average households

\begin{tabular}{|c|c|c|c|c|c|c|}
\hline & \multicolumn{6}{|c|}{ DEPENDENT VARIABLE HOUSEHOLD EXPENDITURES $(\log )$} \\
\hline & $\begin{array}{c}\text { Total } \\
\text { Expenditure }\end{array}$ & $\begin{array}{c}\text { Food } \\
\text { Consumption }\end{array}$ & $\begin{array}{c}\text { Non Food } \\
\text { Consumption }\end{array}$ & $\begin{array}{c}\text { Education } \\
\text { Expenses }\end{array}$ & $\begin{array}{l}\text { Non-Food } \\
\text { Frequent }\end{array}$ & $\begin{array}{l}\text { Non-Food } \\
\text { Less }\end{array}$ \\
\hline Drought $t_{0}(1 / 0)$ & $\begin{array}{c}0.0156 \\
(0.0096)\end{array}$ & $\begin{array}{c}0.0158 \\
(0.0122)\end{array}$ & $\begin{array}{c}0.0258 \\
(0.0168)\end{array}$ & $\begin{array}{c}-0.1746^{* * *} \\
(0.0328)\end{array}$ & $\begin{array}{c}-0.0214 \\
(0.0266)\end{array}$ & $\begin{array}{c}-0.0092 \\
(0.0327)\end{array}$ \\
\hline Drought $t_{1}(1 / 0)$ & $\begin{array}{c}-0.0096 \\
(0.0139)\end{array}$ & $\begin{array}{c}0.0104 \\
(0.0056)\end{array}$ & $\begin{array}{c}-0.0198 \\
(0.0405)\end{array}$ & $\begin{array}{c}-0.1361^{* *} \\
(0.0410)\end{array}$ & $\begin{array}{c}-0.0178 \\
(0.0454)\end{array}$ & $\begin{array}{c}0.2014^{* * *} \\
(0.0406)\end{array}$ \\
\hline Drought $t_{2}(1 / 0)$ & $\begin{array}{l}0.0053^{* *} \\
(0.0016)\end{array}$ & $\begin{array}{l}0.0217^{* *} \\
(0.0054)\end{array}$ & $\begin{array}{c}0.0003 \\
(0.0116)\end{array}$ & $\begin{array}{c}-0.0099 \\
(0.0282)\end{array}$ & $\begin{array}{l}0.1031^{* *} \\
(0.0295)\end{array}$ & $\begin{array}{c}0.0618^{*} \\
(0.0230)\end{array}$ \\
\hline Drought $t_{3}(1 / 0)$ & $\begin{array}{l}-0.0235^{*} \\
(0.0089)\end{array}$ & $\begin{array}{c}-0.0334^{* * *} \\
(0.0051)\end{array}$ & $\begin{array}{c}-0.0023 \\
(0.0183)\end{array}$ & $\begin{array}{c}0.0355 \\
(0.0331)\end{array}$ & $\begin{array}{c}-0.0718 \\
(0.0349)\end{array}$ & $\begin{array}{c}-0.0391 \\
(0.0198)\end{array}$ \\
\hline Household size & $\begin{array}{l}-0.0015 \\
(0.0007)\end{array}$ & $\begin{array}{c}-0.0079 * * * \\
(0.0011)\end{array}$ & $\begin{array}{c}0.0064^{* * *} \\
(0.0013)\end{array}$ & $\begin{array}{c}0.0198 \\
(0.0112)\end{array}$ & $\begin{array}{c}0.0606^{* * *} \\
(0.0025)\end{array}$ & $\begin{array}{c}0.0358^{* * *} \\
(0.0014)\end{array}$ \\
\hline Move out & $\begin{array}{c}-0.0027 \\
(0.0036)\end{array}$ & $\begin{array}{c}0.0117 \\
(0.0138)\end{array}$ & $\begin{array}{c}0.0028 \\
(0.0167)\end{array}$ & $\begin{array}{c}0.0677 \\
(0.0500)\end{array}$ & $\begin{array}{c}0.0585^{*} \\
(0.0248)\end{array}$ & $\begin{array}{c}-0.0063 \\
(0.0243)\end{array}$ \\
\hline Own a farmland & $\begin{array}{l}-0.0066 \\
(0.0086)\end{array}$ & $\begin{array}{l}-0.0037 \\
(0.0074)\end{array}$ & $\begin{array}{l}-0.0268^{*} \\
(0.0107)\end{array}$ & $\begin{array}{l}0.1729 * * \\
(0.0404)\end{array}$ & $\begin{array}{c}-0.1157^{* *} \\
(0.0350)\end{array}$ & $\begin{array}{c}0.0178 \\
(0.0300)\end{array}$ \\
\hline Run a farm & $\begin{array}{c}-0.0058 \\
(0.0042)\end{array}$ & $\begin{array}{c}-0.0139 * * \\
(0.0045)\end{array}$ & $\begin{array}{l}0.0298^{* *} \\
(0.0099)\end{array}$ & $\begin{array}{c}0.2091^{* * *} \\
(0.0197)\end{array}$ & $\begin{array}{c}0.0257 \\
(0.0446)\end{array}$ & $\begin{array}{c}-0.1069^{* * *} \\
(0.0120)\end{array}$ \\
\hline Rich Assets & $\begin{array}{c}-0.0065^{* *} \\
(0.0018)\end{array}$ & $\begin{array}{c}-0.0128 \\
(0.0178)\end{array}$ & $\begin{array}{c}-0.0049 \\
(0.0246)\end{array}$ & $\begin{array}{c}-0.3540^{* * *} \\
(0.0292)\end{array}$ & $\begin{array}{c}0.0026 \\
(0.0279)\end{array}$ & $\begin{array}{c}0.1582^{* * *} \\
(0.0247)\end{array}$ \\
\hline Receive wage & $\begin{array}{c}0.0045 \\
(0.0062)\end{array}$ & $\begin{array}{c}0.0095 \\
(0.0109)\end{array}$ & $\begin{array}{c}-0.0023 \\
(0.0100)\end{array}$ & $\begin{array}{c}-0.1112 \\
(0.0569)\end{array}$ & $\begin{array}{c}0.0001 \\
(0.0186)\end{array}$ & $\begin{array}{l}0.0957^{* *} \\
(0.0246)\end{array}$ \\
\hline Age of head & $\begin{array}{c}0.0007^{* * *} \\
(0.0001)\end{array}$ & $\begin{array}{c}0.0023^{* * *} \\
(0.0002)\end{array}$ & $\begin{array}{l}-0.0002 \\
(0.0006)\end{array}$ & $\begin{array}{c}-0.0039^{* *} \\
(0.0009)\end{array}$ & $\begin{array}{c}-0.0034^{* *} \\
(0.0010)\end{array}$ & $\begin{array}{c}-0.0027 \\
(0.0017)\end{array}$ \\
\hline Head' & $\begin{array}{l}0.0138^{* *} \\
(0.0035)\end{array}$ & $\begin{array}{c}-0.0022 \\
(0.0105)\end{array}$ & $\begin{array}{l}0.0635^{* *} \\
(0.0171)\end{array}$ & $\begin{array}{c}0.0935 \\
(0.0699)\end{array}$ & $\begin{array}{l}0.1426^{* *} \\
(0.0410)\end{array}$ & $\begin{array}{l}0.1136^{* *} \\
(0.0316)\end{array}$ \\
\hline Educ (Uni) & $\begin{array}{c}0.0082 \\
(0.0134)\end{array}$ & $\begin{array}{c}-0.0102 \\
(0.0291)\end{array}$ & $\begin{array}{l}0.0513^{* *} \\
(0.0151)\end{array}$ & $\begin{array}{c}-0.0507 \\
(0.0653)\end{array}$ & $\begin{array}{c}-0.0638 \\
(0.0630)\end{array}$ & $\begin{array}{c}0.0901^{*} \\
(0.0372)\end{array}$ \\
\hline Educ (High) & $\begin{array}{c}0.0254 \\
(0.0137)\end{array}$ & $\begin{array}{l}-0.0110 \\
(0.0121)\end{array}$ & $\begin{array}{c}0.0505^{*} \\
(0.0188)\end{array}$ & $\begin{array}{c}0.1199 \\
(0.1562)\end{array}$ & $\begin{array}{l}-0.0031 \\
(0.0417)\end{array}$ & $\begin{array}{c}0.0172 \\
(0.0421)\end{array}$ \\
\hline Educ (Junior) & $\begin{array}{c}-0.0006 \\
(0.0086)\end{array}$ & $\begin{array}{c}0.0652^{* * *} \\
(0.0058)\end{array}$ & $\begin{array}{c}-0.0638^{* *} \\
(0.0188)\end{array}$ & $\begin{array}{c}0.1293 \\
(0.0927)\end{array}$ & $\begin{array}{c}0.0645 \\
(0.0800)\end{array}$ & $\begin{array}{c}-0.0781^{* *} \\
(0.0208)\end{array}$ \\
\hline Educ (Elemen) & $\begin{array}{c}-0.0046 \\
(0.0026)\end{array}$ & $\begin{array}{l}-0.0043 \\
(0.0147)\end{array}$ & $\begin{array}{c}0.0033 \\
(0.0204)\end{array}$ & $\begin{array}{c}-0.2973^{* * *} \\
(0.0294)\end{array}$ & $\begin{array}{c}-0.1127 \\
(0.0556)\end{array}$ & $\begin{array}{c}0.0459^{*} \\
(0.0196)\end{array}$ \\
\hline Can read and write & $\begin{array}{c}-0.0063 \\
(0.0075)\end{array}$ & $\begin{array}{c}0.0022 \\
(0.0110)\end{array}$ & $\begin{array}{c}-0.0232 \\
(0.0148)\end{array}$ & $\begin{array}{l}0.1505^{* *} \\
(0.0372)\end{array}$ & $\begin{array}{c}-0.0230 \\
(0.0365)\end{array}$ & $\begin{array}{c}0.0263 \\
(0.0446)\end{array}$ \\
\hline$N$ & 5,592 & 5,554 & 5,554 & 3,667 & 5,563 & 5,563 \\
\hline Adj. R2 & 0.0090 & 0.0157 & 0.0152 & 0.0975 & 0.1314 & 0.1277 \\
\hline
\end{tabular}

Note: Driscoll and Kraay standard errors (in parentheses) are robust to heteroscedasticity and potential bias (due to error's spatial and temporal dependences). ${ }^{*} p<0.10,{ }^{* *} p<0.05,{ }^{* * *} p<0.01$ denote statistical significance for the relationship. Drought at IFLS year refers to droughts that occurred in 1993, 1997, 2000, 2007 and 2014. Drought (t-1) refers to droughts that occurred in 1992, 1996, 1999, 2006 and 2013. Drought (t-2) refers to droughts that occurred in 1991, 1995, 1998, 2005 and 2012. Drought (t-3) refers to droughts that occurred in 1990, 1994, 1997, 2004 and 2011. Models are panel household and time fixed-effects with control variables such as household's time-varying characteristics: size, whether own farmland, farm value $>10 \mathrm{~m}$, receive wages, total assets $>100 \mathrm{~m}$, and head's profile (age, gender, education). Rich is defined as a socio-economic condition when household monthly expenditure falls in the highest quantile (the $\left.5^{\text {th }}\right)$. 
While results for poor and average households show varying drought timing that could adversely affect expenditures, results for rich households in Table 11c indicate that takes around three years for a drought to be associated with declines in expenditures. The positive correlations between a contemporaneous drought and several expenditure outcomes may suggest the resilience of rich group against weather shocks in the shortrun. Further, the estimates indicate that rich households do not experience any effect on education expenses, while there are mixed results for total monthly spending, food, and non-food expenditures.

Table 11c Impact of droughts on the monthly expenditure of rich households

\begin{tabular}{|c|c|c|c|c|c|c|}
\hline & \multicolumn{6}{|c|}{ DEPENDENT VARIABLE HOUSEHOLD EXPENDITURES (log) } \\
\hline & $\begin{array}{c}\text { Total } \\
\text { Expenditure }\end{array}$ & $\begin{array}{c}\text { Food } \\
\text { Consumption }\end{array}$ & $\begin{array}{c}\text { Non Food } \\
\text { Consumption }\end{array}$ & $\begin{array}{l}\text { Education } \\
\text { Expenses }\end{array}$ & $\begin{array}{l}\text { Non-Food } \\
\text { Frequent }\end{array}$ & $\begin{array}{c}\text { Non-Food } \\
\text { Less }\end{array}$ \\
\hline Drought $t_{0}(1 / 0)$ & $\begin{array}{c}0.0352^{* *} \\
(0.0097)\end{array}$ & $\begin{array}{c}0.1140^{* *} \\
(0.0248)\end{array}$ & $\begin{array}{c}-0.0073 \\
(0.0283)\end{array}$ & $\begin{array}{c}0.0132 \\
(0.0231)\end{array}$ & $\begin{array}{l}0.1560^{* * *} \\
(0.0290)\end{array}$ & $\begin{array}{l}0.1416^{* * *} \\
(0.0211)\end{array}$ \\
\hline Drought $t_{1}(1 / 0)$ & $\begin{array}{c}-0.0361 \\
(0.0267)\end{array}$ & $\begin{array}{c}-0.0280 \\
(0.0288)\end{array}$ & $\begin{array}{c}-0.0069 \\
(0.0253)\end{array}$ & $\begin{array}{c}0.0507 \\
(0.0571)\end{array}$ & $\begin{array}{c}-0.0181 \\
(0.0365)\end{array}$ & $\begin{array}{c}0.0250 \\
(0.0405)\end{array}$ \\
\hline Drought $t_{2}(1 / 0)$ & $\begin{array}{c}0.0445^{*} \\
(0.0182)\end{array}$ & $\begin{array}{l}0.0762^{* * *} \\
(0.0122)\end{array}$ & $\begin{array}{c}0.0528 \\
(0.0320)\end{array}$ & $\begin{array}{c}0.0193 \\
(0.0372)\end{array}$ & $\begin{array}{l}0.0963^{* *} \\
(0.0254)\end{array}$ & $\begin{array}{c}0.0888 \\
(0.0428)\end{array}$ \\
\hline Drought $t_{3}(1 / 0)$ & $\begin{array}{l}-0.0724^{* *} \\
(0.0250)\end{array}$ & $\begin{array}{c}-0.0746^{* * *} \\
(0.0145)\end{array}$ & $\begin{array}{c}-0.0746 \\
(0.0406)\end{array}$ & $\begin{array}{c}-0.0367 \\
(0.0716)\end{array}$ & $\begin{array}{l}-0.1123^{* *} \\
(0.0276)\end{array}$ & $\begin{array}{c}-0.1709^{* * *} \\
(0.0352)\end{array}$ \\
\hline Household size & $\begin{array}{c}-0.0541^{* * *} \\
(0.0009)\end{array}$ & $\begin{array}{c}-0.0700^{* * *} \\
(0.0026)\end{array}$ & $\begin{array}{c}-0.0414^{* * *} \\
(0.0007)\end{array}$ & $\begin{array}{l}-0.0329^{*} \\
(0.0142)\end{array}$ & $\begin{array}{c}-0.0200 \\
(0.0105)\end{array}$ & $\begin{array}{l}-0.0215^{* *} \\
(0.0062)\end{array}$ \\
\hline Move out & $\begin{array}{c}-0.0271 \\
(0.0164)\end{array}$ & $\begin{array}{c}-0.0506^{* *} \\
(0.0124)\end{array}$ & $\begin{array}{c}0.0123 \\
(0.0095)\end{array}$ & $\begin{array}{c}0.1351^{* *} \\
(0.0441)\end{array}$ & $\begin{array}{c}0.0758^{* *} \\
(0.0247)\end{array}$ & $\begin{array}{c}0.0501 \\
(0.0477)\end{array}$ \\
\hline Own a farmland & $\begin{array}{c}0.0083 \\
(0.0232)\end{array}$ & $\begin{array}{c}0.0468^{* *} \\
(0.0154)\end{array}$ & $\begin{array}{c}0.0114 \\
(0.0439)\end{array}$ & $\begin{array}{c}-0.0124 \\
(0.0458)\end{array}$ & $\begin{array}{c}0.0294 \\
(0.0450)\end{array}$ & $\begin{array}{c}0.1359^{*} \\
(0.0611)\end{array}$ \\
\hline Run a farm & $\begin{array}{l}-0.0271 \\
(0.0318)\end{array}$ & $\begin{array}{c}-0.0454 \\
(0.0258)\end{array}$ & $\begin{array}{c}-0.0054 \\
(0.0446)\end{array}$ & $\begin{array}{c}0.1546 \\
(0.0861)\end{array}$ & $\begin{array}{c}-0.0093 \\
(0.0520)\end{array}$ & $\begin{array}{l}-0.0630 \\
(0.0369)\end{array}$ \\
\hline Rich 1 & $\begin{array}{l}-0.0151 \\
(0.0140)\end{array}$ & $\begin{array}{c}-0.0350 \\
(0.0215)\end{array}$ & $\begin{array}{c}-0.0019 \\
(0.0158)\end{array}$ & $\begin{array}{c}-0.0356 \\
(0.0406)\end{array}$ & $\begin{array}{c}0.0147 \\
(0.0523)\end{array}$ & $\begin{array}{l}-0.0471 \\
(0.0275)\end{array}$ \\
\hline Receive wage & $\begin{array}{c}-0.0229 \\
(0.0111)\end{array}$ & $\begin{array}{c}-0.0088 \\
(0.0139)\end{array}$ & $\begin{array}{l}-0.0759^{* *} \\
(0.0211)\end{array}$ & $\begin{array}{c}-0.0776 \\
(0.0461)\end{array}$ & $\begin{array}{c}-0.1383^{* * *} \\
(0.0256)\end{array}$ & $\begin{array}{c}-0.0029 \\
(0.0420)\end{array}$ \\
\hline Age of head & $\begin{array}{c}-0.0015 \\
(0.0008)\end{array}$ & $\begin{array}{l}-0.0022^{*} \\
(0.0009)\end{array}$ & $\begin{array}{c}-0.0017 \\
(0.0015)\end{array}$ & $\begin{array}{c}-0.0008 \\
(0.0019)\end{array}$ & $\begin{array}{c}-0.0041 \\
(0.0022)\end{array}$ & $\begin{array}{c}-0.0068^{* *} \\
(0.0021)\end{array}$ \\
\hline Head's gender & $\begin{array}{c}-0.1389^{* * *} \\
(0.0143)\end{array}$ & $\begin{array}{c}-0.1182^{* * *} \\
(0.0041)\end{array}$ & $\begin{array}{c}-0.1403^{* * *} \\
(0.0285)\end{array}$ & $\begin{array}{c}-0.1177 \\
(0.1410)\end{array}$ & $\begin{array}{l}-0.1300^{* *} \\
(0.0331)\end{array}$ & $\begin{array}{c}-0.1856^{* * *} \\
(0.0378)\end{array}$ \\
\hline Educ (Uni) & $\begin{array}{c}0.0100 \\
(0.0169)\end{array}$ & $\begin{array}{c}0.0309^{*} \\
(0.0134)\end{array}$ & $\begin{array}{c}-0.0503 \\
(0.0297)\end{array}$ & $\begin{array}{c}0.0852 \\
(0.0481)\end{array}$ & $\begin{array}{c}-0.0040 \\
(0.0512)\end{array}$ & $\begin{array}{c}-0.0842 \\
(0.0689)\end{array}$ \\
\hline Educ (High) & $\begin{array}{c}0.0139 \\
(0.0246)\end{array}$ & $\begin{array}{c}0.0752^{*} \\
(0.0273)\end{array}$ & $\begin{array}{c}-0.0137 \\
(0.0186)\end{array}$ & $\begin{array}{c}-0.0676 \\
(0.0500)\end{array}$ & $\begin{array}{l}0.1794^{* *} \\
(0.0593)\end{array}$ & $\begin{array}{c}0.0697 \\
(0.0474)\end{array}$ \\
\hline Educ (Junior) & $\begin{array}{l}-0.0124 \\
(0.0316)\end{array}$ & $\begin{array}{c}-0.0565 \\
(0.0350)\end{array}$ & $\begin{array}{c}0.0611 \\
(0.0357)\end{array}$ & $\begin{array}{c}0.2267 \\
(0.1281)\end{array}$ & $\begin{array}{c}-0.0526 \\
(0.0735)\end{array}$ & $\begin{array}{l}-0.1378^{* *} \\
(0.0414)\end{array}$ \\
\hline Educ (Elemen) & $\begin{array}{l}-0.0168 \\
(0.0277)\end{array}$ & $\begin{array}{c}-0.0022 \\
(0.0180)\end{array}$ & $\begin{array}{l}-0.0585^{*} \\
(0.0258)\end{array}$ & $\begin{array}{l}-0.3438^{* *} \\
(0.0986)\end{array}$ & $\begin{array}{l}-0.1615^{* *} \\
(0.0576)\end{array}$ & $\begin{array}{c}0.0572 \\
(0.0703)\end{array}$ \\
\hline Can read and write & $\begin{array}{l}-0.0257^{*} \\
(0.0111)\end{array}$ & $\begin{array}{c}-0.0371 \\
(0.0275)\end{array}$ & $\begin{array}{c}-0.0050 \\
(0.0195)\end{array}$ & $\begin{array}{l}0.2386^{* * *} \\
(0.0203)\end{array}$ & $\begin{array}{l}-0.0100 \\
(0.0218)\end{array}$ & $\begin{array}{c}0.0070 \\
(0.0232)\end{array}$ \\
\hline$N$ & 5,592 & 5,515 & 5,405 & 7 & 5,515 & 5,465 \\
\hline
\end{tabular}


\begin{tabular}{lllllll} 
Adj. R2 & 0.0501 & 0.0529 & 0.0282 & 0.0825 & 0.1140 & 0.1199 \\
\hline
\end{tabular}

Note: Driscoll and Kraay standard errors (in parentheses) are robust to heteroscedasticity and potential bias (due to error's spatial and temporal dependences). ${ }^{*} p<0.10,{ }^{* *} p<0.05,{ }^{* * *} p<0.01$ denote statistical significance for the relationship. Drought at IFLS year refers to droughts that occurred in 1993, 1997, 2000, 2007 and 2014. Drought (t-1) refers to droughts that occurred in 1992, 1996, 1999, 2006 and 2013. Drought (t-2) refers to droughts that occurred in 1991, 1995, 1998, 2005 and 2012. Drought (t-3) refers to droughts that occurred in 1990, 1994, 1997, 2004 and 2011. Models are panel household and time fixed-effects. Rich is defined as a socio-economic condition when household monthly expenditure falls in the highest quantile (the $5^{\text {th }}$ ).

\subsubsection{Effects of drought based on its severity}

Finally, we estimate the effects of drought on households by classifying the exogenous shock based on three severity level (recall section 3.3.1 for moderate, severe and extreme drought definitions). The results in earlier models (Table 8 and 9) suggest significant drought-economic nexus, here we analyse further which type of drought affects most the outcomes. We include all intensity-based droughts as main "regressors" to predict changes in the economic outcomes.

\subsubsection{Consequences of moderate, severe and extreme droughts on income}

Table 12 provides detailed results of income models with respect to drought's severity level. It appears that extreme drought affecting incomes the most, shown by greater coefficients in all significant results than any other types of drought. A moderate drought, albeit it has a smaller effect than major droughts, is important for policy attention. Households who experienced a moderate drought would face significant consequences on their agricultural incomes (see column Farm Income and Selling Farm Assets). We don't find correlations between moderate drought and variations in other incomes. To a lower extent than extreme drought, severe drought episodes do affect all type of incomes (see row sev_droL0 to row sev_droL3). They are highly correlated with substantial decreases in predicted total income and non-farm income, where the estimated effects on total income last for a few years after the drought occurred. When it comes to the effects on non-farm income, it appears only severe and extreme drought show significant results.

Other findings indicate the instantaneous effects of drought, for example, on agriculturalrelated incomes. All type of droughts occurred at present year are negatively associated with changes in farm income. The more severe the drought, the greater the impact (see estimated coefficients of mod_droL0, sev_droL0, and ext_droL0 that increase as severity elevates). Further, there are strong correlations between drought episodes and predicted changes in income from selling farm assets. Very significant impacts seem to take effect 2 years after the drought occurred (except in severe drought that shows an immediate effect). The results provide further insights into the findings of earlier estimations 
(section 3.4.1.1) regarding the consequences of a drought that might affect farm activities.

Table 12 Effects of moderate, severe and extreme drought on annual income

\begin{tabular}{|c|c|c|c|c|c|}
\hline & \multicolumn{5}{|c|}{ DEPENDENT VARIABLE: HOUSEHOLD INCOMES (log) } \\
\hline & Total Income & Farm Income & $\begin{array}{l}\text { Non-Farm } \\
\text { Income }\end{array}$ & $\begin{array}{l}\text { Selling Farm } \\
\text { Assets }\end{array}$ & $\begin{array}{l}\text { Selling All } \\
\text { Type of } \\
\text { Assets }\end{array}$ \\
\hline \multirow[t]{2}{*}{ mod_droL0 } & -0.0446 & $-0.0354^{* *}$ & 0.0225 & 0.1321 & -0.1051 \\
\hline & $(0.0302)$ & $(0.0119)$ & $(0.0435)$ & $(0.0845)$ & $(0.1135)$ \\
\hline \multirow[t]{2}{*}{ mod_droL1 } & 0.0215 & $-0.1548^{*}$ & -0.1294 & -0.2298 & -0.0315 \\
\hline & $(0.0665)$ & $(0.0608)$ & $(0.0913)$ & $(0.1767)$ & $(0.2681)$ \\
\hline \multirow[t]{2}{*}{ mod_droL2 } & 0.0294 & 0.0928 & 0.0724 & $0.2417^{*}$ & -0.0409 \\
\hline & $(0.0701)$ & $(0.0875)$ & $(0.0479)$ & $(0.0875)$ & $(0.0511)$ \\
\hline \multirow[t]{2}{*}{ mod_droL3 } & 0.0341 & -0.0468 & 0.0436 & $0.3806^{* * *}$ & $-0.3350^{* *}$ \\
\hline & $(0.0455)$ & $(0.0780)$ & $(0.0369)$ & $(0.0724)$ & $(0.0936)$ \\
\hline \multirow[t]{2}{*}{ sev_droL0 } & $-0.0587^{* *}$ & $-0.0798^{*}$ & 0.1348 & $0.1922^{*}$ & 0.0127 \\
\hline & $(0.0196)$ & $(0.0365)$ & $(0.0711)$ & $(0.0890)$ & $(0.1059)$ \\
\hline \multirow[t]{2}{*}{ sev_droL1 } & $-0.0995^{* * *}$ & 0.0186 & $-0.3727^{* * *}$ & 0.2395 & -0.1003 \\
\hline & $(0.0158)$ & $(0.0447)$ & $(0.0787)$ & $(0.1668)$ & $(0.1239)$ \\
\hline \multirow[t]{2}{*}{ sev_droL2 } & -0.0474 & -0.0694 & -0.0646 & 0.0822 & 0.0583 \\
\hline & $(0.0339)$ & $(0.0751)$ & $(0.0648)$ & $(0.1093)$ & $(0.1609)$ \\
\hline \multirow[t]{2}{*}{ sev_droL3 } & $-0.1361^{* * *}$ & 0.0299 & $-0.3240^{* * *}$ & 0.0148 & $0.4239^{*}$ \\
\hline & $(0.0208)$ & $(0.0680)$ & $(0.0629)$ & $(0.1000)$ & $(0.1554)$ \\
\hline \multirow[t]{2}{*}{ ext_droL0 } & $-0.3769^{* *}$ & $-0.4122^{* *}$ & -0.0380 & -1.2389 & -0.3533 \\
\hline & $(0.0882)$ & $(0.1047)$ & $(0.1103)$ & $(0.9903)$ & $(0.2280)$ \\
\hline \multirow[t]{2}{*}{ ext_droL1 } & 0.0773 & 0.1444 & $-0.5408^{* * *}$ & -0.2478 & -0.2885 \\
\hline & $(0.1429)$ & $(0.1472)$ & $(0.0870)$ & $(0.1715)$ & $(0.3717)$ \\
\hline \multirow[t]{2}{*}{ ext_droL2 } & 0.0061 & 0.0858 & -0.0288 & $0.3026^{* * *}$ & -0.1298 \\
\hline & $(0.0077)$ & $(0.0790)$ & $(0.0169)$ & $(0.0217)$ & $(0.0703)$ \\
\hline \multirow[t]{2}{*}{ ext_droL3 } & $0.2519^{* *}$ & 0.1146 & -0.0185 & $2.1615^{* * *}$ & $0.7043^{* *}$ \\
\hline & $(0.0813)$ & $(0.1016)$ & $(0.1018)$ & $(0.1551)$ & $(0.1706)$ \\
\hline \multirow[t]{2}{*}{ hhsize } & $-0.0511^{* * *}$ & $-0.0498^{* * *}$ & $-0.0732^{* * *}$ & $-0.0727^{* * *}$ & $-0.0745^{* * *}$ \\
\hline & $(0.0046)$ & $(0.0029)$ & $(0.0069)$ & $(0.0108)$ & $(0.0112)$ \\
\hline \multirow[t]{2}{*}{ outsubmove } & 0.0470 & $-0.0491^{* *}$ & 0.0428 & 0.1627 & 0.0392 \\
\hline & $(0.0233)$ & $(0.0140)$ & $(0.0278)$ & $(0.1083)$ & $(0.0349)$ \\
\hline \multirow[t]{2}{*}{ farmland } & 0.0515 & -0.0704 & 0.0027 & -0.0409 & $-0.0853^{*}$ \\
\hline & $(0.0512)$ & $(0.0468)$ & $(0.0076)$ & $(0.0566)$ & $(0.0397)$ \\
\hline \multirow[t]{2}{*}{ ownfarm } & $0.3129^{* * *}$ & $0.4953^{* * *}$ & -0.0144 & $0.4597^{* * *}$ & $0.2626^{* * *}$ \\
\hline & $(0.0352)$ & $(0.0380)$ & $(0.0086)$ & $(0.0441)$ & $(0.0555)$ \\
\hline \multirow[t]{2}{*}{ richasset } & $0.3483^{* * *}$ & $0.3240^{* * *}$ & $0.2740^{* * *}$ & $0.4228^{* * *}$ & $0.3588^{* * *}$ \\
\hline & $(0.0363)$ & $(0.0269)$ & $(0.0109)$ & $(0.0790)$ & $(0.0489)$ \\
\hline \multirow[t]{2}{*}{ labour } & $1.1732^{* * *}$ & $0.4544^{* * *}$ & $0.6801^{* * *}$ & $0.5118^{* * *}$ & $0.2395^{* * *}$ \\
\hline & $(0.1221)$ & $(0.0287)$ & $(0.0788)$ & $(0.0562)$ & $(0.0519)$ \\
\hline \multirow[t]{2}{*}{ poor } & 0.0000 & 0.0000 & 0.0000 & 0.0000 & 0.0000 \\
\hline & (.) & (.) & (.) & (.) & $()$. \\
\hline \multirow[t]{2}{*}{ quantile 2} & -0.0014 & $0.0420^{*}$ & -0.0337 & $0.2278^{* * *}$ & -0.0599 \\
\hline & $(0.0128)$ & $(0.0189)$ & $(0.0458)$ & $(0.0469)$ & $(0.0549)$ \\
\hline \multirow[t]{2}{*}{ average } & -0.0112 & -0.0270 & -0.0048 & 0.1143 & $0.1039^{* *}$ \\
\hline & $(0.0054)$ & $(0.0138)$ & (0.0319) & $(0.0612)$ & $(0.0330)$ \\
\hline quantile 4 & 0.0450 & $0.0849^{* * *}$ & $0.0954^{*}$ & 0.1821 & $0.1184^{* *}$ \\
\hline & $(0.0224)$ & $(0.0136)$ & $(0.0368)$ & $(0.0922)$ & $(0.0337)$ \\
\hline ricl & $0.0962^{* * *}$ & $0.0902^{* * *}$ & $0.0800^{*}$ & 0.0248 & $0.1227^{* *}$ \\
\hline
\end{tabular}




\begin{tabular}{lccccc} 
& $(0.0198)$ & $(0.0138)$ & $(0.0292)$ & $(0.0598)$ & $(0.0299)$ \\
ageHead & $0.0028^{* *}$ & $-0.0012^{* * *}$ & $-0.0052^{* * *}$ & 0.0039 & -0.0010 \\
& $(0.0007)$ & $(0.0003)$ & $(0.0009)$ & $(0.0019)$ & $(0.0017)$ \\
head_man & 0.0013 & 0.0105 & $-0.1318^{* *}$ & 0.2331 & -0.0150 \\
& $(0.0406)$ & $(0.0154)$ & $(0.0340)$ & $(0.1765)$ & $(0.0795)$ \\
univ & -0.0326 & $-0.1190^{* *}$ & 0.0122 & -0.0033 & $-0.0776^{*}$ \\
& $(0.0261)$ & $(0.0263)$ & $(0.0159)$ & $(0.1037)$ & $(0.0315)$ \\
hischool & -0.0521 & 0.0020 & $-0.1172^{* *}$ & 0.1865 & -0.0035 \\
& $(0.0280)$ & $(0.0279)$ & $(0.0371)$ & $(0.0989)$ & $(0.0500)$ \\
juniorhi & $0.0687^{* * *}$ & 0.0165 & $0.0874^{* *}$ & -0.0110 & 0.0561 \\
& $(0.0125)$ & $(0.0327)$ & $(0.0259)$ & $(0.1734)$ & $(0.0796)$ \\
element & 0.0400 & -0.0125 & 0.0624 & -0.0808 & -0.0546 \\
& $(0.0344)$ & $(0.0364)$ & $(0.0511)$ & $(0.1561)$ & $(0.0389)$ \\
literate & $0.1753^{* * *}$ & 0.0448 & 0.0518 & 0.0420 & 0.1436 \\
& $(0.0270)$ & $(0.0216)$ & $(0.0458)$ & $(0.1694)$ & $(0.0811)$ \\
\hline$N$ & 23,626 & 12,511 & 10,929 & 2,638 & 7,443 \\
Adj. R2 & 0.5049 & 0.3672 & 0.2799 & 0.1385 & 0.1720 \\
\hline
\end{tabular}

Note: Driscoll and Kraay standard errors (in parentheses) are robust to heteroscedasticity and potential bias (due to error's spatial and temporal dependences). ${ }^{*} p<0.10,{ }^{* *} p<0.05,{ }^{* * *} p<0.01$ denote statistical significance for the relationship. Models include household and time fixed-effects. Moderate drought refers to spei values between -1.49 and -1 in 6 consecutive months in a year or between -1.49 and -1 in 4 consecutive rainy season months. Severe drought refers to spei values between -1.99 and -1.5 in 6 consecutive months in a year or between -1.99 and -1.5 in 4 consecutive rainy season months. And, extreme drought refers to spei values below -2 in 6 consecutive months in a year or below -2 in 4 consecutive rainy season months. Main predictors: mod_droL $0=$ moderate drought at present year, mod_droL1= moderate drought last year and so on. Further, sev_droL0 refers to severity drought at present year, ext_droL0 refers to extreme drought at present year, and so on.

\subsubsection{Impacts on household expenditures based on drought severity}

The results in Table 13 indicate higher associations between extreme drought and predicted changes in household spending, as opposed to moderate and severe droughts. These confirm earlier findings of income models. In the expenditure models, extreme drought has not only greater impacts (bigger coefficients than other droughts when they are statistically significant) but also more significant results than 2 other droughts. If there was an extreme drought this year, for example, households experienced this drought would have to cut non-food expenditures by $6.5 \%$ (see column Non-Food Expenditure and row ext_droL0). While, on the other hand, other types of drought (moderate and severe) do not show adverse effects on this spending (see column NonFood Expenditure). The estimated effects of extreme drought are also evident in spending on education (large effects, see Table 13 column education, row droughtL1, and L3) and food (to a lesser extent, see column food expenditure, row ext_droL3). An extreme drought occurred last year is associated with a large drop in education expenditure up to $21.5 \%$. Lastly, a lower severity drought (moderate or severe drought) seems to affect little monthly expenditures of households who experienced drought shocks. A severe drought 
last year, however, needs watchful attention because, based on our estimations, it predicts a $20 \%$ cut in households' education spending.

Table 13 Effects of drought on household expenditures relative to its severity

\begin{tabular}{|c|c|c|c|c|c|c|}
\hline & \multicolumn{6}{|c|}{ DEPENDENT VARIABLE HOUSEHOLD EXPENDITURES (log) } \\
\hline & $\begin{array}{c}\text { Total } \\
\text { Expenditure }\end{array}$ & $\begin{array}{c}\text { Food } \\
\text { Expenditure }\end{array}$ & $\begin{array}{c}\text { Non Food } \\
\text { Expenditure }\end{array}$ & $\begin{array}{l}\text { Education } \\
\text { Expenditure }\end{array}$ & $\begin{array}{l}\text { Non-Food } \\
\text { Frequent }\end{array}$ & $\begin{array}{l}\text { Non-Food } \\
\text { Less }\end{array}$ \\
\hline mod_droL0 & $\begin{array}{c}0.0008 \\
(0.0016)\end{array}$ & $\begin{array}{c}-0.0026 \\
(0.0089)\end{array}$ & $\begin{array}{c}0.0060 \\
(0.0057)\end{array}$ & $\begin{array}{c}-0.0067 \\
(0.0162)\end{array}$ & $\begin{array}{c}0.0018 \\
(0.0161)\end{array}$ & $\begin{array}{c}-0.0119 \\
(0.0251)\end{array}$ \\
\hline mod_droL1 & $\begin{array}{l}-0.0141^{* *} \\
(0.0031)\end{array}$ & $\begin{array}{c}0.0071 \\
(0.0161)\end{array}$ & $\begin{array}{c}0.0061 \\
(0.0274)\end{array}$ & $\begin{array}{c}-0.0423 \\
(0.0206)\end{array}$ & $\begin{array}{c}-0.0531 \\
(0.0259)\end{array}$ & $\begin{array}{c}0.1079^{*} \\
(0.0427)\end{array}$ \\
\hline mod_droL2 & $\begin{array}{c}0.0174 \\
(0.0112)\end{array}$ & $\begin{array}{l}0.0371^{* * *} \\
(0.0040)\end{array}$ & $\begin{array}{c}-0.0051 \\
(0.0103)\end{array}$ & $\begin{array}{c}0.0183 \\
(0.0210)\end{array}$ & $\begin{array}{c}0.0215 \\
(0.0224)\end{array}$ & $\begin{array}{c}0.0196 \\
(0.0135)\end{array}$ \\
\hline mod_droL3 & $\begin{array}{c}-0.0084 \\
(0.0059)\end{array}$ & $\begin{array}{c}-0.0070 \\
(0.0043)\end{array}$ & $\begin{array}{c}-0.0022 \\
(0.0077)\end{array}$ & $\begin{array}{c}0.0083 \\
(0.0357)\end{array}$ & $\begin{array}{c}-0.0151 \\
(0.0123)\end{array}$ & $\begin{array}{c}-0.0002 \\
(0.0101)\end{array}$ \\
\hline sev_droL0 & $\begin{array}{l}0.0414^{* * *} \\
(0.0086)\end{array}$ & $\begin{array}{l}0.0478^{* * *} \\
(0.0087)\end{array}$ & $\begin{array}{c}0.0248^{*} \\
(0.0102)\end{array}$ & $\begin{array}{c}0.0178 \\
(0.0366)\end{array}$ & $\begin{array}{c}0.0609 \\
(0.0446)\end{array}$ & $\begin{array}{c}0.0765 \\
(0.0494)\end{array}$ \\
\hline sev_droL1 & $\begin{array}{c}0.0048 \\
(0.0094)\end{array}$ & $\begin{array}{c}-0.0220 \\
(0.0117)\end{array}$ & $\begin{array}{c}0.0425^{* *} \\
(0.0145)\end{array}$ & $\begin{array}{c}-0.2000^{* * *} \\
(0.0372)\end{array}$ & $\begin{array}{c}-0.0280 \\
(0.0489)\end{array}$ & $\begin{array}{c}-0.0150 \\
(0.0167)\end{array}$ \\
\hline sev_droL2 & $\begin{array}{l}0.0166^{* *} \\
(0.0041)\end{array}$ & $\begin{array}{c}-0.0076 \\
(0.0120)\end{array}$ & $\begin{array}{l}0.0492^{* * *} \\
(0.0093)\end{array}$ & $\begin{array}{c}0.0450 \\
(0.0598)\end{array}$ & $\begin{array}{c}0.0189 \\
(0.0166)\end{array}$ & $\begin{array}{l}0.0777^{* *} \\
(0.0210)\end{array}$ \\
\hline sev_droL3 & $\begin{array}{l}-0.0233^{*} \\
(0.0125)\end{array}$ & $\begin{array}{l}-0.0271^{* *} \\
(0.0072)\end{array}$ & $\begin{array}{c}-0.0155 \\
(0.0206)\end{array}$ & $\begin{array}{c}0.0728 \\
(0.0347)\end{array}$ & $\begin{array}{l}-0.0328 \\
(0.0310)\end{array}$ & $\begin{array}{l}-0.0027 \\
(0.0385)\end{array}$ \\
\hline ext_droL0 & $\begin{array}{c}-0.0282 \\
(0.0140)\end{array}$ & $\begin{array}{c}-0.0181 \\
(0.0205)\end{array}$ & $\begin{array}{c}-0.0649^{* * *} \\
(0.0046)\end{array}$ & $\begin{array}{c}0.1634 \\
(0.0906)\end{array}$ & $\begin{array}{c}-0.1453^{* * *} \\
(0.0204)\end{array}$ & $\begin{array}{c}-0.1055^{* * *} \\
(0.0186)\end{array}$ \\
\hline ext_droL1 & $\begin{array}{c}0.0238 \\
(0.0205)\end{array}$ & $\begin{array}{c}0.0471 \\
(0.0295)\end{array}$ & $\begin{array}{l}-0.0141^{*} \\
(0.0066)\end{array}$ & $\begin{array}{c}-0.2146^{* * *} \\
(0.0293)\end{array}$ & $\begin{array}{c}0.0891^{* *} \\
(0.0209)\end{array}$ & $\begin{array}{c}0.0542^{* *} \\
(0.0173)\end{array}$ \\
\hline ext_droL2 & $\begin{array}{c}0.0060 \\
(0.0044)\end{array}$ & $\begin{array}{c}0.0055 \\
(0.0054)\end{array}$ & $\begin{array}{c}0.0109 \\
(0.0083)\end{array}$ & $\begin{array}{c}0.0100 \\
(0.0495)\end{array}$ & $\begin{array}{c}0.0383^{* *} \\
(0.0083)\end{array}$ & $\begin{array}{l}0.0939^{* * *} \\
(0.0088)\end{array}$ \\
\hline ext_droL3 & $\begin{array}{c}0.0102 \\
(0.0109)\end{array}$ & $\begin{array}{l}-0.0376^{*} \\
(0.0151)\end{array}$ & $\begin{array}{l}0.0754^{* * *} \\
(0.0026)\end{array}$ & $\begin{array}{l}-0.2067^{*} \\
(0.0872)\end{array}$ & $\begin{array}{c}0.0304 \\
(0.0295)\end{array}$ & $\begin{array}{c}0.0038 \\
(0.0258)\end{array}$ \\
\hline hhsize & $\begin{array}{c}-0.0186^{* * *} \\
(0.0008)\end{array}$ & $\begin{array}{c}-0.0271^{* * *} \\
(0.0006)\end{array}$ & $\begin{array}{c}-0.0105^{* * *} \\
(0.0012)\end{array}$ & $\begin{array}{l}0.0072^{* * *} \\
(0.0011)\end{array}$ & $\begin{array}{l}0.0161^{* * *} \\
(0.0018)\end{array}$ & $\begin{array}{l}0.0084^{* * *} \\
(0.0015)\end{array}$ \\
\hline outsubmove & $\begin{array}{c}0.0063^{* *} \\
(0.0016)\end{array}$ & $\begin{array}{c}0.0081 \\
(0.0076)\end{array}$ & $\begin{array}{c}0.0082 \\
(0.0043)\end{array}$ & $\begin{array}{c}0.0391 \\
(0.0274)\end{array}$ & $\begin{array}{c}0.0180^{* *} \\
(0.0046)\end{array}$ & $\begin{array}{c}-0.0011 \\
(0.0086)\end{array}$ \\
\hline farmland & $\begin{array}{c}-0.0005 \\
(0.0017)\end{array}$ & $\begin{array}{c}0.0012 \\
(0.0029)\end{array}$ & $\begin{array}{l}-0.0111^{* *} \\
(0.0031)\end{array}$ & $\begin{array}{c}0.0175 \\
(0.0160)\end{array}$ & $\begin{array}{c}0.0115 \\
(0.0090)\end{array}$ & $\begin{array}{c}0.0144 \\
(0.0116)\end{array}$ \\
\hline ownfarm & $\begin{array}{l}-0.0085^{*} \\
(0.0031)\end{array}$ & $\begin{array}{c}-0.0102 \\
(0.0070)\end{array}$ & $\begin{array}{c}0.0046 \\
(0.0028)\end{array}$ & $\begin{array}{c}0.0183 \\
(0.0184)\end{array}$ & $\begin{array}{c}-0.0199 \\
(0.0165)\end{array}$ & $\begin{array}{l}-0.0206 \\
(0.0135)\end{array}$ \\
\hline richasset & $\begin{array}{c}-0.0048 \\
(0.0036)\end{array}$ & $\begin{array}{c}-0.0077 \\
(0.0068)\end{array}$ & $\begin{array}{c}0.0007 \\
(0.0046)\end{array}$ & $\begin{array}{l}-0.0485^{* *} \\
(0.0134)\end{array}$ & $\begin{array}{c}0.0148 \\
(0.0145)\end{array}$ & $\begin{array}{c}-0.0003 \\
(0.0193)\end{array}$ \\
\hline labour & $\begin{array}{c}-0.0012 \\
(0.0046)\end{array}$ & $\begin{array}{c}0.0078 \\
(0.0073)\end{array}$ & $\begin{array}{c}-0.0170^{* * *} \\
(0.0025)\end{array}$ & $\begin{array}{c}0.0031 \\
(0.0072)\end{array}$ & $\begin{array}{c}0.0112^{*} \\
(0.0047)\end{array}$ & $\begin{array}{c}-0.0092 \\
(0.0164)\end{array}$ \\
\hline poor & $\begin{array}{c}0.0000 \\
\text { (.) }\end{array}$ & $\begin{array}{c}0.0000 \\
\text { (.) }\end{array}$ & $\begin{array}{c}0.0000 \\
(.)\end{array}$ & $\begin{array}{c}0.0000 \\
(.)\end{array}$ & $\begin{array}{c}0.0000 \\
(.)\end{array}$ & $\begin{array}{c}0.0000 \\
(.)\end{array}$ \\
\hline quantile 2 & $\begin{array}{l}0.7989^{* * *} \\
(0.0309)\end{array}$ & $\begin{array}{l}0.7635^{* * *} \\
(0.0265)\end{array}$ & $\begin{array}{l}0.8128^{* * *} \\
(0.0373)\end{array}$ & $\begin{array}{l}0.9990^{* * *} \\
(0.0473)\end{array}$ & $\begin{array}{l}1.1224^{* * *} \\
(0.0572)\end{array}$ & $\begin{array}{l}1.0608^{* * *} \\
(0.0463)\end{array}$ \\
\hline average & $\begin{array}{l}1.3367^{* * *} \\
(0.0329)\end{array}$ & $\begin{array}{l}1.2583^{* * *} \\
(0.0215)\end{array}$ & $\begin{array}{l}1.4025^{* * *} \\
(0.0488)\end{array}$ & $\begin{array}{l}1.7317^{* * *} \\
(0.0533)\end{array}$ & $\begin{array}{l}1.9243^{* * *} \\
(0.0748)\end{array}$ & $\begin{array}{l}1.7477^{* * *} \\
(0.0353)\end{array}$ \\
\hline quantile 4 & $\begin{array}{l}1.9300^{* * *} \\
(0.0324)\end{array}$ & $\begin{array}{l}1.7897^{* * *} \\
(0.0223)\end{array}$ & $\begin{array}{l}2.0699^{* * *} \\
(0.0483)\end{array}$ & $\begin{array}{l}2.5507^{* * *} \\
(0.0552)\end{array}$ & $\begin{array}{l}2.8235^{* * *} \\
(0.0578)\end{array}$ & $\begin{array}{l}2.5657^{* * *} \\
(0.0503)\end{array}$ \\
\hline
\end{tabular}




\begin{tabular}{lcccccc} 
rich & $2.9133^{* * *}$ & $2.6511^{* * *}$ & $3.1389^{* * *}$ & $3.7536^{* * *}$ & $4.0665^{* * *}$ & $3.7946^{* * *}$ \\
ageHead & $(0.0175)$ & $(0.0090)$ & $(0.0277)$ & $(0.0202)$ & $(0.0170)$ & $(0.0169)$ \\
& $0.0006^{* * *}$ & $0.0009^{* * *}$ & 0.0004 & 0.0002 & 0.0001 & -0.0004 \\
head_man & $(0.0001)$ & $(0.0001)$ & $(0.0002)$ & $(0.0006)$ & $(0.0003)$ & $(0.0003)$ \\
& $-0.0307^{* * *}$ & $-0.0319^{* * *}$ & $-0.0204^{*}$ & $-0.0737^{* *}$ & -0.0086 & 0.0047 \\
univ & $(0.0029)$ & $(0.0028)$ & $(0.0084)$ & $(0.0217)$ & $(0.0197)$ & $(0.0189)$ \\
& -0.0075 & -0.0044 & -0.0071 & 0.0340 & 0.0112 & 0.0025 \\
hischool & $(0.0049)$ & $(0.0035)$ & $(0.0054)$ & $(0.0337)$ & $(0.0137)$ & $(0.0249)$ \\
& 0.0013 & 0.0099 & -0.0088 & $-0.0865^{* * *}$ & -0.0439 & -0.0188 \\
juniorhi & $(0.0072)$ & $(0.0068)$ & $(0.0112)$ & $(0.0127)$ & $(0.0351)$ & $(0.0217)$ \\
& $0.0233^{* * *}$ & $0.0199^{*}$ & $0.0263^{* *}$ & $0.1635^{* * *}$ & 0.0641 & 0.0080 \\
element & $(0.0035)$ & $(0.0076)$ & $(0.0067)$ & $(0.0106)$ & $(0.0320)$ & $(0.0104)$ \\
& $-0.0107^{*}$ & 0.0001 & $-0.0206^{* *}$ & $-0.0850^{* * *}$ & -0.0054 & -0.0128 \\
literate & $(0.0045)$ & $(0.0062)$ & $(0.0069)$ & $(0.0184)$ & $(0.0143)$ & $(0.0123)$ \\
& $-0.0176^{* *}$ & $-0.0118^{* *}$ & -0.0205 & -0.0207 & -0.0230 & -0.0195 \\
& $(0.0048)$ & $(0.0033)$ & $(0.0114)$ & $(0.0128)$ & $(0.0115)$ & $(0.0137)$ \\
\hline$N$ & 27,945 & 27,666 & 27,551 & 18,145 & 27,663 & 27,635 \\
Adj. R2 & 0.8836 & 0.7930 & 0.7885 & 0.5250 & 0.6960 & 0.6269 \\
\hline
\end{tabular}

Note: Driscoll and Kraay standard errors (in parentheses) are robust to heteroscedasticity and potential bias (due to error's spatial and temporal dependences). ${ }^{*} p<0.10,{ }^{* *} p<0.05,{ }^{* * *} p<0.01$ denote statistical significance for the relationship. Models include household and time fixed-effects. Moderate drought refers to spei values between -1.49 and -1 in 6 consecutive months in a year or between -1.49 and -1 in 4 consecutive rainy season months. Severe drought refers to spei values between -1.99 and -1.5 in 6 consecutive months in a year or between -1.99 and -1.5 in 4 consecutive rainy season months. And, extreme drought refers to spei values below -2 in 6 consecutive months in a year or below -2 in 4 consecutive rainy season months. Main predictors: mod_droL0=moderate drought at present year, mod_droL1= moderate drought last year and so on. Further, sev_droL0 refers to severity drought at present year, ext_droL0 refers to extreme drought at present year, and so on.

Beside these negative effects, our results in expenditure models also show a number of positive relationships between drought and expenditures. These might reflect not only the impact factor of severity level (because most positive signs are caused by either moderate or severe drought) but also the effectivity of coping mechanisms during each drought's type 33 .

\subsection{Conclusion}

In this paper, we estimate the impact of drought in the immediate three years after it occurs. We find robust evidence of the adverse effects of drought on households, where the scale and significance of the impact depend on several characteristics. Our findings indicate that the impacts are significant on household incomes only after a drought occurred the year before. We find larger impacts on household incomes (between 7\% and $42 \%$ changes) relative to impacts on household consumption (between $1 \%$ and $9 \%$

\footnotetext{
${ }^{33}$ For example, with regards to helps and supports from family/friend/relatives as well as from community or government are quite common during weather shocks. The increased expenditures (positive signs of the coefficients) indicate external assistances might help reduce the effect of moderate and severe drought episodes (but probably not the extreme ones).
} 
percentage changes). Selling farm assets is apparently the preferred coping mechanism used to respond to the drought shock. Overall, the findings suggest entrepreneurshipstyle activities are likely the most highly vulnerable incomes affected by drought shocks.

The drought effects evidently vary between one household group and another (here we analysed for the poor, average and rich households). Interestingly, our findings suggest no income impact for poor although there are significant adverse effects on expenditures for food, non-food and education. Compared to other groups, average households experience larger effect on incomes from the farm and non-farm business. The statistically significant results come from contemporaneous droughts (drought this year and last year). The same droughts also predict substantial decreases in spending on education of average households, which are greater than any other groups. For rich households, there are mixed results with respect to income's type and drought's timing. But there is a clear line suggesting that the effects on this group are only significantly negative in 3 years after the drought occurred and the impacts are larger than on other groups -especially, on non-farm income and on non-food expenditure. Overall, it can be concluded that in the case of impact on non-farm income, the large negative effects reflect severe consequences to average and rich household groups.

The severity of drought seems to matter much in terms of its impact on incomes and monthly expenditures. As might be expected, extreme droughts have much higher significant associations with decreases in all income and expenditure models, than moderate and severe droughts do. However, the lower severity droughts still merit policy attention. Our results suggest that a severe drought affects all incomes and predicts a $20 \%$ cut in spending on education spending. Even moderate droughts still have significant consequences on agricultural-related incomes. 


\section{Annexes Chapter THREE}

Annex 1 Agro-climatic Oldeman 1901 - 1950, 1950 - 1980 and 1980 - 2015

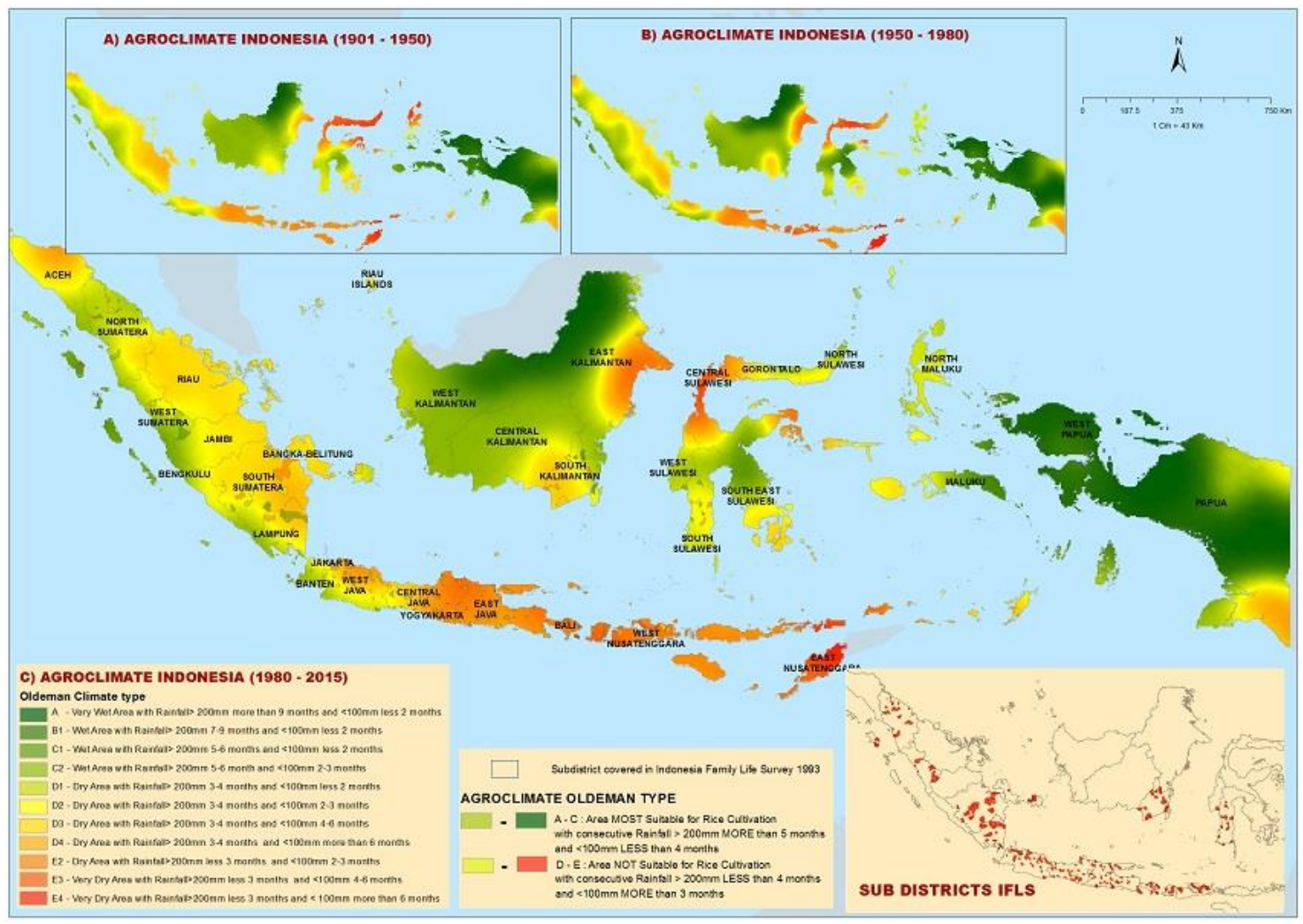

Sources: Author's calculation on the precipitation climatic data 1901 - 2015 obtained from the Climate Research Unit (CRU) of the University of East Anglia

Annex 2 Impact of Weather Shocks on Indonesia (1990 - 2017)

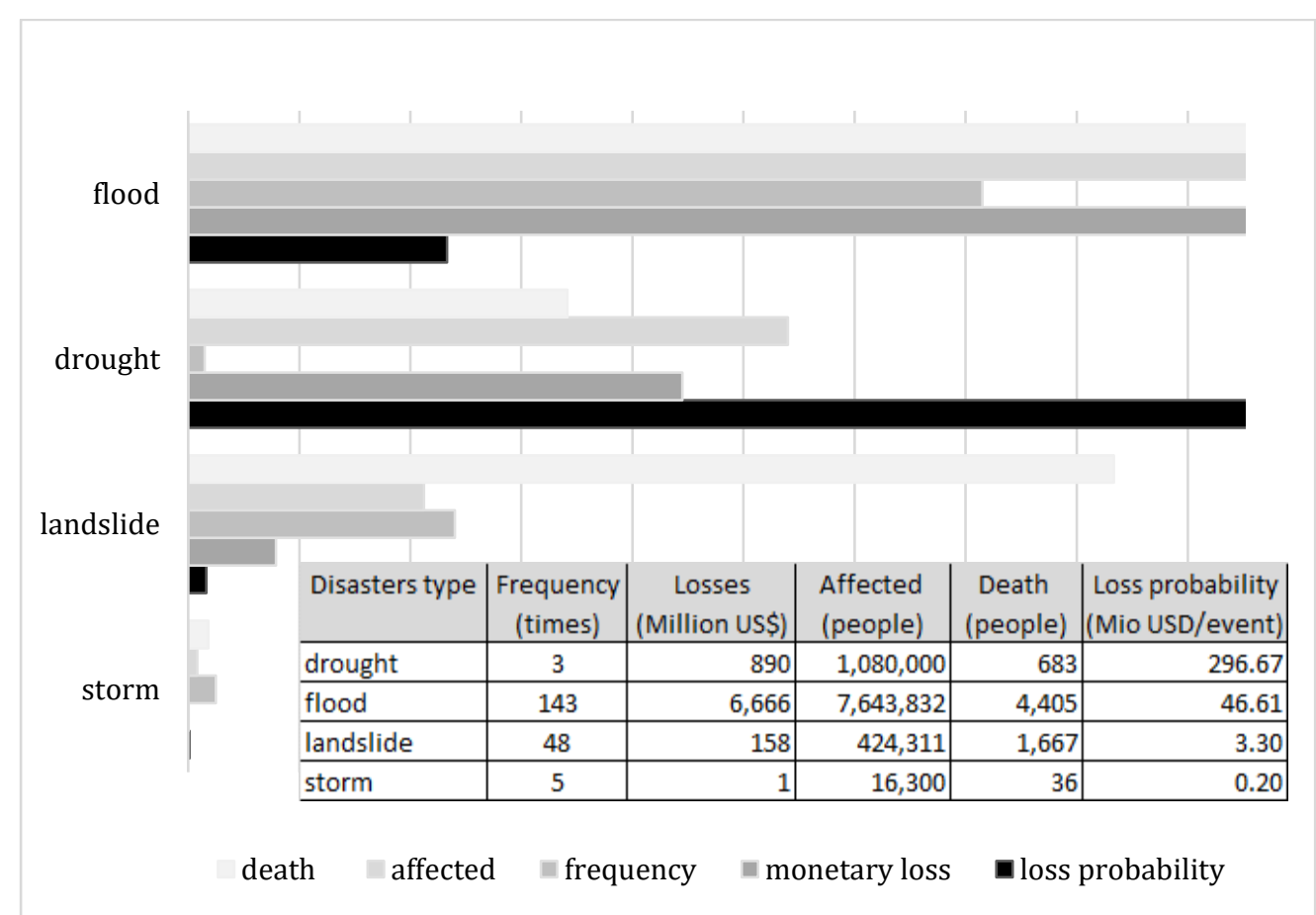

Source: Author's calculation from EM-DAT. Access date: 5 June 2018 
Annex 3 Total Monetary Damages and Number of People Affected or Killed due to Weather Shocks in Indonesia (1990 - 2007)

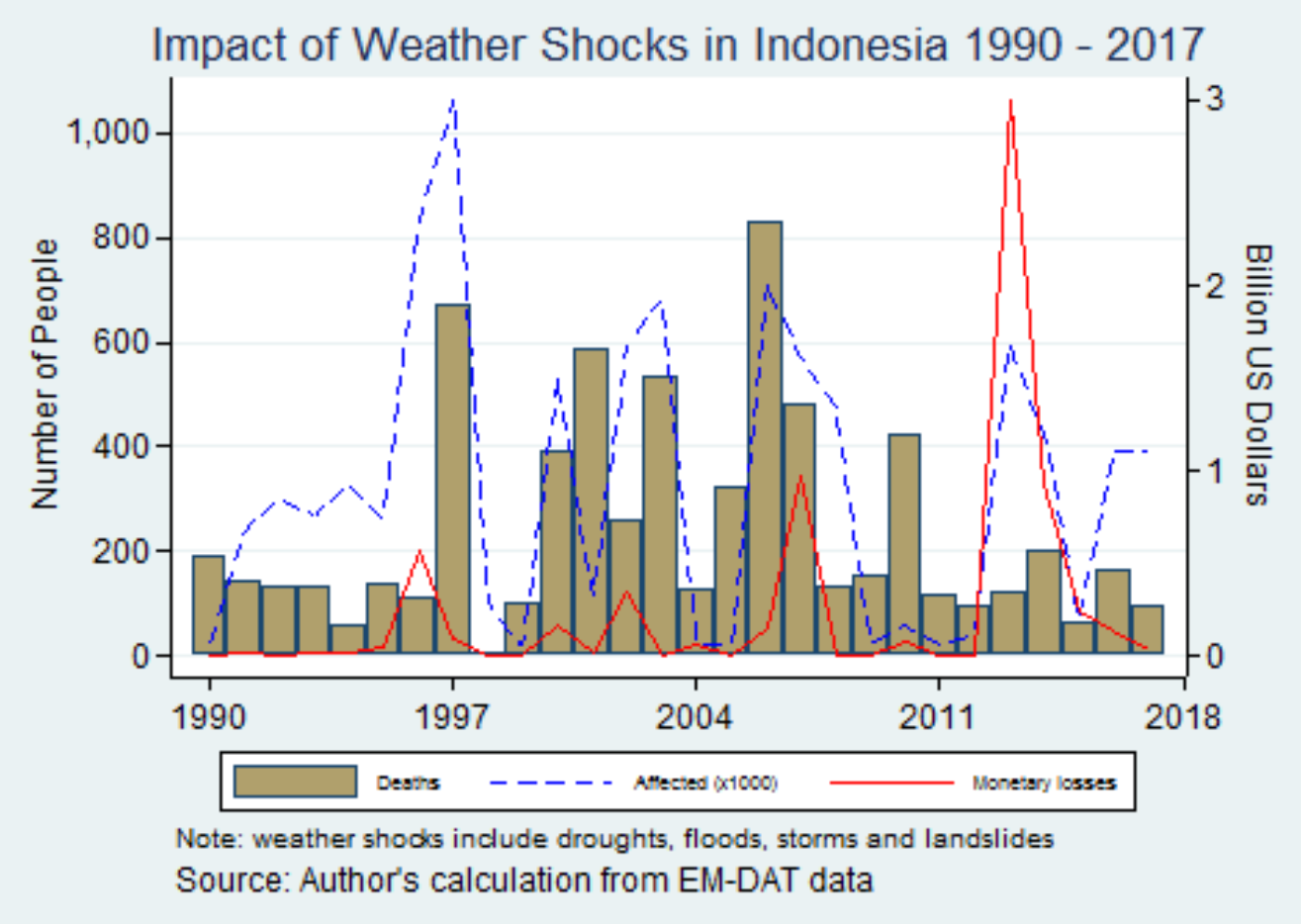

Source: Author's calculation from EM-DAT

Annex 4 Drought events in selected areas based on the author's drought method

\begin{tabular}{|c|c|c|c|c|c|}
\hline YEAR & BINAMU $^{1}$ & SAKRA $^{2}$ & PASAWAHAN $^{3}$ & PORSEA $^{4}$ & NOTES \\
\hline 1990 & - & - & - & - & \multirow{6}{*}{$\begin{array}{l}\text { 1. Sub-district } \\
\text { Binamu, district } \\
\text { Jeneponto, South } \\
\text { Sulawesi }\end{array}$} \\
\hline 1991 & Drought & - & - & - & \\
\hline 1992 & - & - & - & - & \\
\hline 1994 & Drought & - & - & - & \\
\hline 1995 & - & - & - & - & \\
\hline 1996 & - & - & - & - & \\
\hline 1997 & Drought & Drought & - & - & \multirow{9}{*}{$\begin{array}{l}\text { 2. Sub-district Sakra, } \\
\text { district Lombok } \\
\text { Timur, West Nusa } \\
\text { Tenggara }\end{array}$} \\
\hline 1998 & Drought & Drought & - & - & \\
\hline 1999 & - & - & - & - & \\
\hline 2004 & - & Drought & - & - & \\
\hline 2005 & Drought & - & - & Drought & \\
\hline 2006 & - & - & - & - & \\
\hline 2011 & - & - & - & - & \\
\hline 2012 & - & - & - & - & \\
\hline 2013 & - & - & - & - & \\
\hline
\end{tabular}


Annex 5 Irrigated Areas in Main Islands Indonesia in 2016

\begin{tabular}{|c|c|c|c|c|c|c|c|c|}
\hline Island & Sumatera & Java & $\begin{array}{c}\text { Bali and } \\
\text { Nusatenggara }\end{array}$ & Kalimantan & Sulawesi & Maluku & Papua & Total \\
\hline Irrigated Sawah (Ha) & $1,061,105$ & $2,417,185$ & 390,944 & 166,266 & 711,360 & 22,277 & 12,358 & $4,781,495$ \\
\hline Total Sawah (Ha) & $2,209,673$ & $3,222,347$ & 533,304 & $1,082,669$ & $1,048,515$ & 27,402 & 62,560 & $8,186,470$ \\
\hline Total Non Sawah (Ha) & $7,437,744$ & $3,049,153$ & $2,163,968$ & $5,460,762$ & $3,345,902$ & $2,173,626$ & $4,946,694$ & $28,577,849$ \\
\hline Total Agricultural areas (Ha) & $9,647,418$ & $6,271,500$ & $2,697,272$ & $6,543,431$ & $4,394,417$ & $2,201,028$ & $5,009,254$ & $36,764,319$ \\
\hline Percentage irrigated areas of national irrigated (\%) & $22.19 \%$ & $50.55 \%$ & $8.18 \%$ & $3.48 \%$ & $14.88 \%$ & $0.47 \%$ & $0.26 \%$ & $100 \%$ \\
\hline Percentage irrigated areas of total sawah (\%) & $48.02 \%$ & $75.01 \%$ & $73.31 \%$ & $15.36 \%$ & $67.84 \%$ & $81.30 \%$ & $19.75 \%$ & $58 \%$ \\
\hline Percentage irrigated areas of total planted (\%) & $14.58 \%$ & $38.91 \%$ & $20.96 \%$ & $5.11 \%$ & $20.51 \%$ & $1.79 \%$ & $0.83 \%$ & $17 \%$ \\
\hline Percentage irrigated areas of total agri areas (\%) & $11.00 \%$ & $38.54 \%$ & $14.49 \%$ & $2.54 \%$ & $16.19 \%$ & $1.01 \%$ & $0.25 \%$ & $13 \%$ \\
\hline
\end{tabular}

Source: Author's calculation from Indonesia's Ministry of Agriculture (http://prasarana.pertanian.go.id/lahanmy/). Access date 3 June 2018

Annex 6 Statistical checks on the total income model specifications using graphs

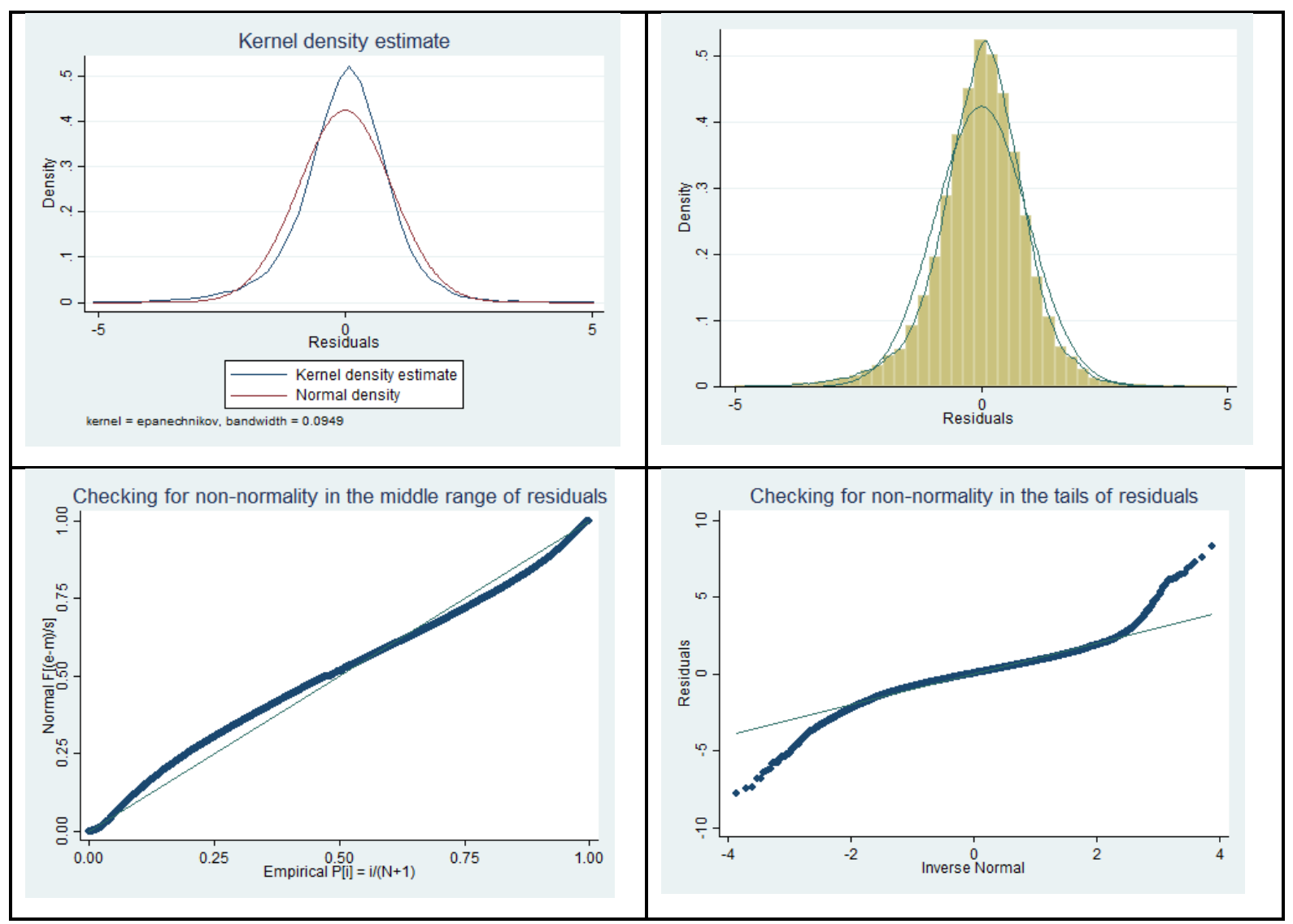




\section{Annex 7 Scatter plots fitted and observed outcome variables}
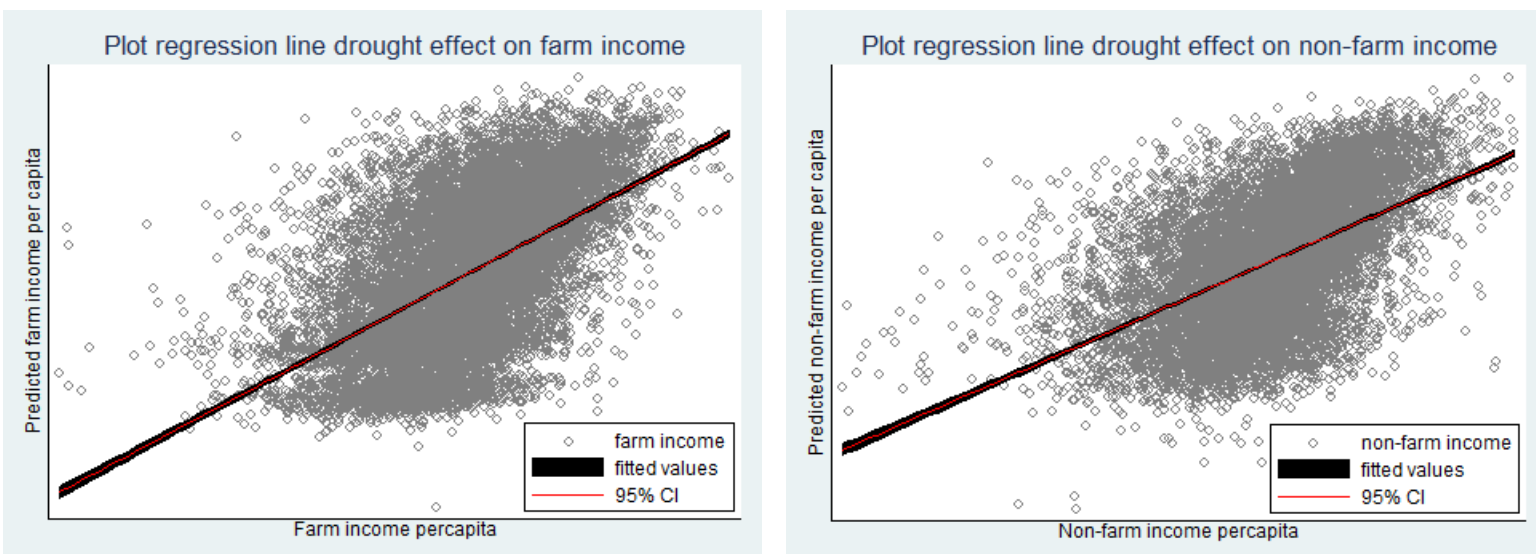

Plot regression line drought effect on total income

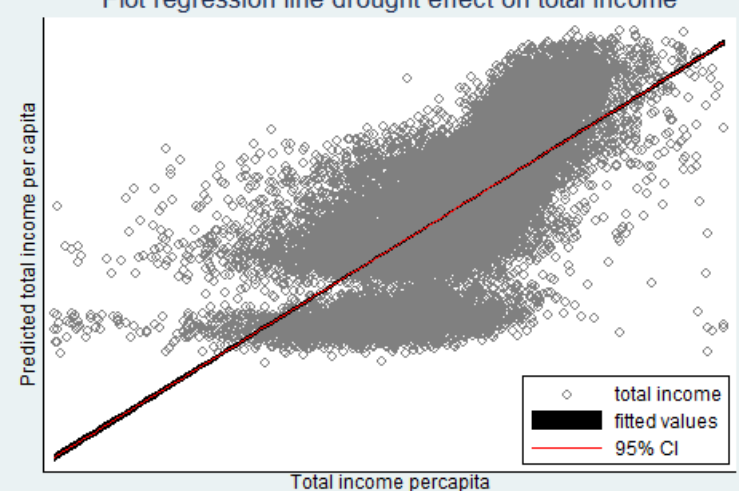

Plot regression line drought effect on total expenditure

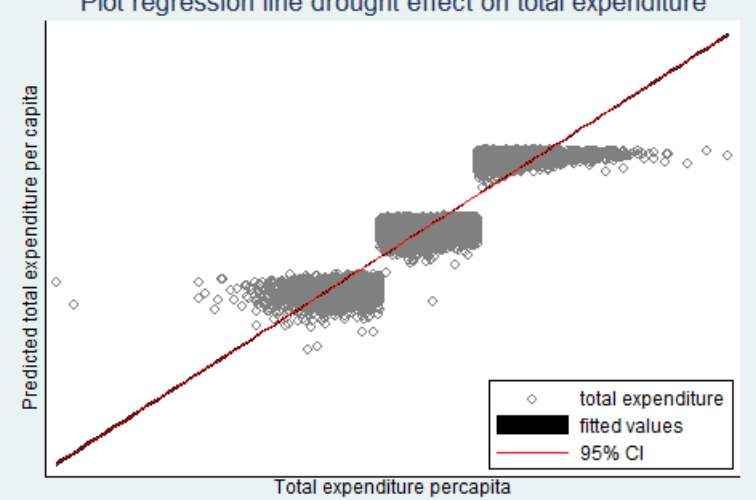




\title{
Chapter FOUR
}

\section{THE EFFECTS OF EXTREME WEATHER ON ADULTS' HEALTH AND WELL-BEING}

\begin{abstract}
:
This study quantifies the effects of experiencing extreme precipitation on health outcomes and well-being of adult individuals in Indonesia. Specifically, it identifies episodes of extreme low and high monthly rainfall, using the historical (1993-2014) rainfall record at sub-district level. The exogenous rainfall shocks are then linked with social, health and economic indicators of a longitudinal sample working age individuals from the 5 waves of the Indonesian Family Life Survey (1993, 1997, 2000, 2007 and 2014). This paper also examines the coping mechanisms through which households attempt to lessen their susceptibility to extreme weather-associated impacts. Our findings suggest that both wet and dry months are significantly correlated with an increased likelihood of having digestive problems. An additional one wet month increases the predicted probability by 1.4 percentage point (pp) and an additional one dry month rises the chance to $0.7 \mathrm{pp}$. Further, the results indicate that there are marginal effects of dry months on health outcomes. Every additional one dry month increases the likelihood of respiratory and mild illnesses (by 0.1 and 0.13 pp respectively). We find mixed results on outpatient health expenditures, a number of days bed-ridden due to illness, visit frequency for outpatient care, self-reported health status, and life satisfaction. The results on coping mechanisms suggest heterogeneous effects with respect to, for example, the role of health insurance.
\end{abstract}




\subsection{Introduction}

Irregular patterns of weather realizations in the last few decades signify a changing climate, which is predicted to exacerbate the frequency and severity of weather events. This motivates a growing number of empirical works that are aimed to improve our understanding of climate-economy impacts, especially in developing countries where weather-related mortality, morbidity, health costs, and overall losses are higher (McIver et al., 2016; Burgess et al., 2017; Karim, 2018; Letta et al., 2018; Yonson, 2018). Researchers consider the tangible effects of weather events on GDP, agricultural losses, variations of household income or expenditure, damage to infrastructure (including buildings and houses). On the other hand, recent empirical works have also examined less easily-monetised effects like loss of human life and health impact, and lately life satisfaction. The latter focus is endorsed in the 2015 Sendai Framework which mandates the inclusion of health as a key indicator, together with sustainable development and climate change issue, in global policy development for disaster risk reduction (DRR) 2015 - 2030 (Aitsi-Selmi et al., 2015).

This paper attempts to make progress in understanding short-term and direct effects of recurring weather shocks on adult's health and well-being using panel household survey data from Indonesia. Reviews about weather-health nexus in economics literature (Dell et al., 2014) and epidemiology (Lo Iacono et al., 2017) suggest that key areas for further advancement in the literature include better identification of causal impacts (because of potential problems of spatial heterogeneity and/or autocorrelations), and connecting longitudinally weather-individual outcomes.

The present study exploits the deviations of monthly rainfall from historic averages and uses these to identify extreme events (above the $90^{\text {th }}$ percentile and below the $10^{\text {th }}$ percentile). The empirical analysis is done at the sub-district level. It then combines these identified weather shocks with a variety of health and wellbeing outcomes of working age individuals. It uses $0.5^{0}$ x $0.5^{\circ}$ gridded weather data of the Climate Research Unit (CRU) of the University of East Anglia and, for the social, health and economic indicators, it uses information gathered from five waves of the Indonesian Family Life Survey (IFLS 1993, 1997, 2000, 2007 and 2014). The outcomes of interests examined include symptoms of illness (mild, digestive, respiratory and infectious), number of days in bed due to illness, number of visits in outpatient care facilities, outpatient costs, self-reported health status, 
and life satisfaction. We further test whether having insurance (through the government scheme or private insurance) and other economic characteristics matter for the health impacts of weather- shock, as earlier work has suggested (see e.g. Lohmann and Lechtenfeld (2015)).

The results suggest that both wet and dry extreme months are leading to changes in the frequency of mild illness symptoms such as a headache, runny nose, sore eyes, cough, cold, and nausea as well as to having digestive problems. There is also a statistically significant correlation between dry months and symptoms of respiratory problems such as difficult breathing, while we don't find causal relations between extreme weather and symptoms of infectious illnesses.

We present less robust results with regards to extreme weather impacts on outpatient expenditures, number of days bed-ridden, number of visits to outpatient care clinics, selfreported health status, and reported happiness. For all of these, intriguingly, the overall picture shows little weather effects.

The contribution of this chapter to the literature is mostly because it connects a long period of spatially detailed weather data and rich longitudinal household survey panel spanning more than 2 decades. This allows the chapter to examine a large selection of health outcomes and well-being while existing studies are typically restricted to a narrow set of outcome variables.

The chapter is further organized as follows. Section two is the discussion of existing literature, while section three explains the methodology and empirical strategy. Then, data and summary statistics are reviewed in section four, section five describes the findings, and section six concludes.

\subsection{Literature review}

Weather is an important part of the daily life of people and its impacts on social and economic activities have long been recognized. Review by Dell et al. (2014) suggests that a negative association between temperature and income has been noted at least as early as the fourteenth century, in Ibn Khaldun's Muqaddimah. Much later works revealed adverse consequences on agriculture, aggregate outputs (i.e. income, expenditure, GDP, GDP growth, industrial and services), energy, conflicts, and health outcomes (see also 
Hsiang et al. (2013), Deschenes (2014), Auffhammer and Schlenker (2014), and FreireGonzález et al. (2017) for more appraisals). While income and expenditure are commonly studied as economic indicators of interest, there is a growing body of literature in economics measuring weather/climate effects using subjective well-being (SWB) variable such as life satisfaction -- which is quite common in psychology literature. Rehdanz and Maddison (2005) was an early study of the weather-SWB link and they found a sound signal that extreme precipitation and temperature can determine to SWB in the observed study area (67 countries).

The increasing evidence of health impacts from environmental change was initially reviewed in the 2007 report of the International Panel on Climate Change Working Group II (see Deschenes (2014) and Haines et al. (2009) for detailed discussions) and further endorsed in the 2015 Sendai Framework for Disaster Risk Reduction (UNISDR, 2015b).

\subsubsection{Extreme Weather and Health}

Literature about weather influence on human health can be traced back in the $17^{\text {th }}$ century when outbreaks of epidemic disease occurred in Europe. It has been argued by many, including (Bull \& Morton, 1975) and Galloway (1985), that environmental conditions, specifically weather variability, increase mortality rates during the preindustrial time in Europe (1600-1800). In an attempt to reveal major determinants of death in the city of London recorded in the Bill of Mortality from 1670 to 1830, Galloway (1985) studied annual variations in prices, temperature, and rainfall on annual fluctuations in age-specific and disease-specific mortality. He found a positive linear relationship between grain prices and deaths of middle and older age groups while abnormally cold winters and unusually warm summers are associated with increased mortality among the elderly.

Galloway's empirical findings are parallel to conclusions of earlier studies (Bull \& Morton, 1975; Bull \& Morton, 1978; Post, 1984). Post (1984) found increased mortality 17401742 in British Isles, France, the Low Countries, the German states, Switzerland, Italy, and Scandinavia corresponding with extreme weather patterns, high food prices and outbreaks of epidemic diseases. The evidence offers two possible channels linking climatic shocks and deaths/health outcomes. First, soaring food prices -- due to bad harvest caused by extreme weather -- increased famine episodes, which affect nutrition intake as well as hygiene and sanitation. Secondly, extreme weather conditions and 
subsequent distress facilitated the transmission of specific pathogens and disease vectors, thereby increasing the morbidity rates of several infectious diseases.

Subsequent works maintain findings that extreme weather has direct implications on health and even death (in case of a hurricane, extreme drought or flash floods) or other physical distress (i.e. thermal stress has a deleterious effect to those with pre-existing respiratory or cardiac disease). The adverse effects are also mediated by indirect mechanisms, such as through infectious agents/vectors or ecological disruption as well as via changes in economic and socio-demographic (see the Patz et al. (2000) and Dell et al. (2014) reviews). Studies also point out that extreme weather effects on individual health outcomes and well-being can be contemporary (Hajat et al., 2005) and long-run (Maccini \& Yang, 2009). Finally, it is important to note that the literature highlights that often it is the vulnerability that determines the severity of weather-disaster impacts.

\subsubsection{The research design in the weather-health literature}

Earlier studies use cross-sectional designs to quantify weather effects on health. Causative inference using this approach is however often challenged by difficulties in identifying true weather effects from other characteristics that might be correlated with the outcomes (Dell et al., 2014) or potential endogeneity problem because other spatially varying unobservable factors may likely be correlated with the observed weather variables (Auffhammer et al., 2013). From the conceptual and methodological point of view, rising use of panel approaches that control for space and time fixed effects in the weather-health link is encouraging as the methods offer straightforward causative inference while requiring relatively few identification assumptions. Fixed-effects estimators offer insights on whether extreme weather deviations in a spatially-specific study area do change the outcome of interest (see Dell et al. (2014) and Auffhammer et al. (2013) for reviews on panel weather-economy models).

Research examining extreme weather and health relationships are essentially focused on either analyzing anomalous variations of weather/climatic variables (i.e. precipitation, temperature, relative humidity, solar radiation, wind speed and direction, etc.) or measuring changes of outcome of interest before and after major weather events such as floods, droughts, heatwaves and storms. While both approaches contribute to further understanding of weather-effects, analyzing rich variations of 'generic' weather variables 
enable the researcher to experiment with presumably exogenous weather shocks on the outcomes. For example, Rehdanz and Maddison (2005) construct various long average weather indices to define climate shocks such as, annually averaged precipitation, annually averaged temperature, mean temperature of the coldest month, mean temperature of the hottest month, mean precipitation of the driest month, mean precipitation of the wettest month, and the number of cold/hot/dry/wet months. Further, 'generic' weather variables such as temperature and precipitation are useful for weather and climate change prediction because most climate models consider these variables as the main inputs (Auffhammer et al., 2013).

The choice of weather variables as appropriate "regressors" for explaining variations of the outcome of interest has been constantly a source of debate. Nevertheless, a general consensus suggests that weather variables may be closely correlated (i.e. precipitation and temperature in the case of extreme events causing deaths and diseases, wind speed and precipitation for storm effects, etc.) -- see reviews of Auffhammer et al. (2013) and Dell et al. (2014). Recent papers use typically more than one variable, be that as main explanatory variable or as the control -- although adoption of panel models may reduce the effect of unbiased estimates of one single weather variable. Overall, research focused on temperature find a high correlation with mortality (Deschenes, 2014; Otrachshenko et al., 2018). While those focused on precipitation find a significant impact on morbidity and other health outcomes such as height (Eisenberg et al., 2013; Groppo \& Kraehnert, 2016).

To this end, review on weather-health studies suggests there are several methodological and conceptual issues in the current literature. Deschenes (2014) points out the issues that may become pitfalls if not addressed appropriately are the measurements of health outcomes, modelling the exposure of weather shock, research design, external validity and the inclusion of adaptation strategies. Many studies, for example, are focused on mortality and hospitalization, as measurements of health outcomes. This has limited present knowledge on possibly considerable other effects weather shock, such as, on the quality of life. The current article attempts to fill the gap by extending the outcomes being studied to several health-related outcomes (morbidity, outpatient visit, bedrest days, etc.) as well as life satisfaction. 
4.2.3 Literature progress and gaps in low and low to middle-income countries

The weather-associated health risks and impacts in developing countries are disproportionally high relative to their size of the economy, livelihood structures, and institutional capability to prepare for and respond to the shocks. These conditions have been reported in many policy notes, including in a Health and Disaster Risk report of the World Health Organization Health (WHO, 2014) and the Global Assessment Report on Disaster Risk Reduction of the United Nations Office for Disaster Risk Reduction (UNISDR, 2015a). In the recent economics literature, Yonson (2018) found that floods increase the probability of urban population in the Philippines to suffer from various diseases. Ultimately, these flood-associated morbidities become very costly for the government and the affected poor families. Further, De Alwis and Noy (2017) estimated that the economic costs of health impacts due to frequently occurring floods and droughts in Sri Lanka reach 19 million USD per year, equally borne by the government and households. Most relevant work to the present analysis is Lohmann and Lechtenfeld (2015)'s panel study which examined extreme rainfall-associated health outcomes and expenditures in Vietnam using panel fixed-effect models. Its findings suggest that drought increases the probability of infectious, non-communicable and long-term illnesses, which in turn affected household health expenditures. Further, it finds that formal and informal insurance mechanisms reduce the effect of drought on health care spending. Its identification strategy, assuming rainfall deficit affects health expenditure only through its link with health shock in the household (drought as an instrument variable), is however different than the current article that examines directly the effect of rainfall shock on a number of health outcomes.

Most research using Subjective Well-Being (SWB) variable, such as life satisfaction or happiness, as a proxy of individual outcomes in measuring weather effects are done in those countries with well-developed capacities to maintain economic and health statistics, and weather data. Rehdanz and Maddison (2005) and Maddison and Rehdanz (2011) looked at the impact of climatic variables on SWB in several countries, including some developing economies. They relate temperature anomalies with SWB relying on global datasets, such as the World Values Survey (WVS) for SWB data which are coupled with World Bank and CIA Factbook socio-economic data. Since this research focusses on cross-country results using aggregated data at the national level, it provides a very 
different approach that is largely unable to account for intra-country heterogeneity (associated with income, location or any other source of within-country difference).

Brereton et al. (2008) is one of the earliest attempts to examine the influence of extreme weather on happiness which considers location-specific factors. Using a cross-sectional approach, it observed annual variations of average precipitation, temperature, sunshine hours and wind speed and found that exogenous weather variables are significantly associated with life satisfaction of 1500 adult individuals living in Ireland in 2000. A recent work of Barrington-Leigh and Behzadnejad (2017) in Canada found that daily variations in weather conditions (temperature, rainfall, snow, and cloud) are associated with changes in SWB, although SWB impact is small relative to socio-economic variables such as income. Its specifications that attach the subject unit being studied with nearby station weather data while also control individual characteristics to account for individual fixed-effects is somewhat related to the present paper that studies the Indonesian setting. However, the present paper does not include control over geographic and seasonal effects.

Empirical studies in economics that examine the influence of weather and/or climate effects in Indonesia are mostly focused on adverse consequences on agricultural, income, welfare and other economic outcomes. There are still limited published research looking at the health impacts. From this narrow reference, much literature suggests that the extremes in weather, especially rainfall variability, are highly correlated with disease incidents, such as vector-borne Malaria and Dengue (Arcari et al., 2007; Rejeki et al., 2018). Maccini and Yang (2009) examined the socio-economic impacts of extreme rainfall in early life for an individual's later life. They found that higher than normal rainfall during infancy has long-term beneficial consequences on individual health status, height, and economic outcomes.

Using a different method than the present paper, Hanandita and Tampubolon (2014) found that rainfall shocks in Indonesia are associated with changes in common mental disorders through its effect on individual welfare. This study basically tests whether economic condition causes poor mental health, by instrumenting the seasonal anomalies in rainfall which evidently determines the poverty status in Indonesia. Interesting to note that a $1 \%$ decrease in consumption expenditures, which in this case due to rainfall shock, 
increase the risk of an individual to have symptoms of common mental disorders by $0.62 \%$.

These findings confirm significant weather-health links in the Indonesian setting, but there remain several gaps. For example, little is known about short-term and direct effects of recurring weather shocks on an individual because none of the published papers using panel method in Indonesia study. Further, there is no empirical work examined potentially large "low effect" health-related outcomes such as mild symptoms of illness or bedrest's days due to weather extreme, which may be possibly related to someone's productivity. Lastly, the SWB (life satisfaction) effects from weather shock have not been studied much in Indonesia. These gaps motivate the present article to advance current understanding through new features, including the application of panel research design using fixed-effects specifications and estimating the effects on a number of health outcomes such as symptoms of illness, outpatient expenditures, number of visit to medical facilities for outpatient care, etc.

\subsection{Methodology and empirical strategy}

This paper is looking specifically at the influence of extreme weather conditions on an individual's health and subjective well-being. Since the weather has spatial and temporal characteristics, it observes the extremes in local weather obtained at the nearby weather station of the adult individuals being surveyed. Specifically, it considers variations in weather conditions in the last three months, including the present month, compared to the extreme rainfall history in the last 50 years. Here, we examine the effect of two types of exogenous precipitation shocks; a wet month and dry month. A wet month episode is identified when actual monthly precipitation exceeds the high historic threshold (calculated at $90^{\text {th }}$ percentile of the monthly record), and a dry month is when actual monthly precipitation falls at or below low historic threshold (calculated at the $10^{\text {th }}$ percentile) ${ }^{34}$. To control potential bias due to omitted weather variable, the hot month

\footnotetext{
34 The historic thresholds (90th and $10^{\text {th }}$ percentile) are based on the same month across year and space
} 
episode is also identified (at the high threshold $90^{\text {th }}$ percentile) ${ }^{35}$. These wet month, dry month and hot month are then used as binary indicators.

The panel approach in the current paper relates the changes of individual health-related indicators in 5 waves of IFLS with local weather variations at the sub-district level, having controlled the time-constant variables. The empirical analysis follows a general model specification below:

$Y_{i h s t}=\alpha_{i}+\beta W_{i s t}+\delta X_{i h t}+\theta_{t}+\varepsilon_{i t}$

where $Y_{i t}$ is a set of dependent variables health and well-being that being examined. It includes symptoms of illness, a number of bedrest day, health expenditure (outpatient care), outpatient visit, health status and happiness (life satisfaction). The symptoms of illness is a set of dichotomous variable health outcomes including mild, digestive, respiratory problems and infectious diseases that an individual $i$ experience in relation to the occurrence of weather extreme in the last three months. $W_{i s}$ is a vector that measures extreme weather episodes that individual $i$ experience in sub-district $s$ at the $t$ th wave of IFLS. $X_{i}$ denotes a vector of an individual and household characteristics, where a number of variables are considered, including age, household size, marriage status, gender, education, house ownership status, has electricity at home, and total monthly household expenditure. We also control for heat, with a binary variable indicating when the maximum monthly temperature is higher than the $90^{\text {th }}$ percentile (based on historical temperature data). $\alpha_{i}$ denotes the panel fixed effects (for individual $i$ ), a set of time fixedeffects, $\theta_{t}$ is also included. Finally, $\varepsilon_{i t}$ refers to error terms which are clustered by subdistrict to allow for arbitrary heteroscedasticity and autocorrelation within sub-districts. The vector of coefficients $\beta$ is the main parameter of interest to be estimated while $\delta$ is the estimated parameters of other independent variables that are associated with the outcomes.

To examine whether some variables interact with exposure to extreme precipitation events, we also include, in some specifications, interaction effects. These include health

\footnotetext{
35 The use of rainfall and temperature as weather shocks are widely applied in climatic-economic study. As they are likely related, excluding one of them may bias the 'true' weather effect (Auffhammer et al., 2013; Dell et al., 2014)
} 
insurance participation (public and private), wealth (total assets), and farm income. A general interaction model is specified as follows:

$$
Y_{i h s t}=\alpha_{i}+\beta W_{i s t}+\gamma M_{i h t}+\varphi\left(W_{i s t} * M_{i h t}\right)+\delta X_{i h t}+\theta_{t}+\varepsilon_{i s t}
$$

where $Y_{i t}$ is a set of dependent variables, which now is limited to only those that can be predicted linearly (health expenditure, outpatient visit, and bedrest). $M$ indexes a vector of control variables that reflects mitigating features of an individual for which these may differ the intensity of weather effects between one group of individual and another. Therefore, the parameter of interest is $\varphi$ which represents the interacting effect of weather's vector $W$ and characteristic $M$ or $\left(W_{i s t} * M_{i h t}\right)$. In this specification, there are 5 (five) variables being tested; such that variables epitomize risk coping strategies in the form of formal and informal insurance. Variables represent formal mechanism are enrolment in government health insurance scheme or in private insurance scheme. Non formal ones include individual's household economic condition (based on monthly expenditure), total assets and farm income. The latter one is included in the coping analysis to check whether households that have low (or none) farm income are less vulnerable to rainfall shocks than the ones that have high farm income. To avoid the direct effect of assets and income on health, the estimations consider the previous survey values (lagged 1 wave).

The outcome variables being measured have different types of data distributions, therefore we use several regression methods. Probit models are fitted for binary symptoms of illness and marginal effects are reported in the result section to provide a more intuitive causal inference. Tobit regressions are used for estimating outpatient cost (semi-continuous), the number of the days for bedrest (discrete), and outpatient visits (discrete). These variables have a large proportion of zero and, specifically for discrete variables, smaller means that are skewed (see descriptive statistics, Table 14 and histograms of these variables in Annex 4-6). For estimating the effects of rainfall shocks on categorical variables (health status and life satisfaction), ordinal regressions (probit) are fitted to model the relationships. 


\subsection{Weather Data and Socioeconomic statistics}

This study uses two main sources of data for the panel analysis. The weather data are obtained from the Climate Research Unit (CRU) of the University of East Anglia and the socioeconomic indicators, including health data, are gathered from 5 waves of the Indonesian Family Life Survey (IFLS 1993, 1997, 2000, 2007 and 2014).

\subsubsection{Gridded weather data}

Classical problems using historical weather data from weather stations are inconsistent measurements, either spatially or temporally, and many missing values in the data. The other challenge to obtain weather station data observed in a long period time in Indonesia is the limited access to this information. Therefore, this study uses freely accessible gridded datasets of CRU's University of East Anglia, United Kingdom. The data are derived through interpolation methods and advanced climate models of weather data collected by the global network of weather stations. Specifically, the current version of CRU TS v. 4.01 (Harris et al., 2014) is used to make a complete panel weather dataset measured at 1,012 climate data points throughout Indonesia. The climate data points are obtained from the global weather data CRU gridded at $0.5^{\circ} \times 0.5^{\circ}$ spatial resolution, which corresponds roughly to $56 \mathrm{~km}$ across every grid cell at the equator. The final dataset consists of 139 climate data point that covers 602 sub-districts (kecamatan) in Indonesia. The climate data points are matched with IFLS areas to link these weather data with every individual survey using his/her known location (sub-district) ${ }^{36}$.

A wet month is defined when that month's rainfall is equal to or exceeds the historic upper extreme ( $90^{\text {th }}$ percentile) and a dry month is, equivalently, when rainfall is equal to or below the historic lower extreme (10 th percentile). The historic extreme thresholds are calculated from the 50 years weather data (each month across the year) at subdistrict level and for the purpose of this study, wet and dry month variables are binary. Further, the actual rainfall variable considers weather realizations in the month of IFLS interview date and the two months before. To this end, the main weather predictors (total wet months and total dry months) used in the empirical analysis are constructed by summing up wet and dry month variables in all three month period of observations. The identification strategy follows a common assumption that the three-month window

\footnotetext{
36 The nearest climate point to the centroid of the sub-districts is used to match the weather record to each household
} 
method may give the best approximation for moisture conditions of the soil as suggested in some studies (McKee, Doesken, \& Kleist, 1995; Sims, Nigoyi, \& Raman, 2002; VicenteSerrano, 2006 in Lohmann and Lechtenfeld (2015)).

The rainfall data vary quite significantly between one region and another in the study areas while temperature differences are not so significant. For example, during the $5^{\text {th }}$ wave of IFLS (surveyed in 2014-2015), a dry month in Sumatera is wetter (with an average rainfall $101 \mathrm{~mm} /$ month at each sub-district) compared to a dry month in East Nusa Tenggara which on average in each sub-district has rainfall $3 \mathrm{~mm} / \mathrm{month}$.
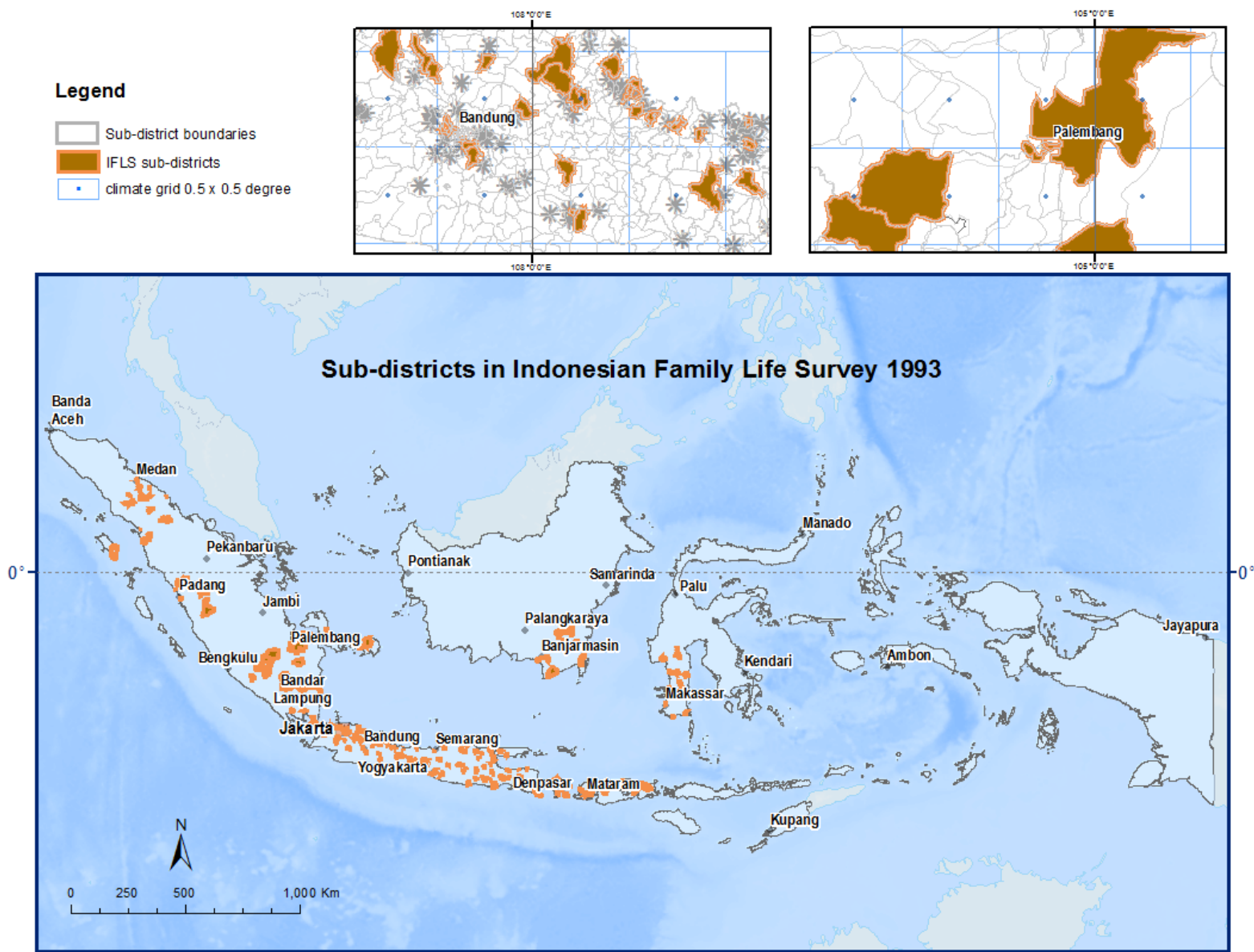

Source: gridded climate data is obtained from the Climate Research Unit of the University of Anglia and IFLS (Indonesian Family Life Survey) sub-districts are located from the first wave (1993).

Figure 9 Study area and gridded weather data

With regards to temperature, the maximum temperature in Sumatera during what we term as a hot month is $33.1^{\circ}$ Celsius, compared to maximum temperature in East Nusa Tenggara that reaches $32.4^{0}$ Celsius. Figure 9 illustrates the coverage area of gridded weather data across sub-districts represented in the IFLS. 


\subsubsection{Socioeconomic indicators of individuals in the IFLS}

The data collection for this study uses a considerable amount of socioeconomic and health information at the individual level from five rounds of the Indonesian Family Life Survey (IFLS). IFLS is an on-going longitudinal survey that has been recording various indicators at individual and households as well as community level since 1993 until the recent wave in 2014. IFLS is claimed to represent $83 \%$ of Indonesian heterogeneous population in the first survey in 1993 (encompassed 13 provinces) and it consistently traces individuals and households involved in the survey. Until the last wave in 2014, IFLS (including IFLS EAST in 2012) have interviewed 16,204 households and 50,148 individuals where the respondents are spread around almost all provinces of Indonesia (28 provinces).

The dependent variables in this study consist of several self-reported health outcomes, including four binary variables that indicate a symptom of illness and three quantifiable variables that inform expenditure for outpatient care (continuous), the number of days of bedrest (discrete) and the number of outpatient visits to medical facilities (discrete). All of these outcomes reflect information for the last 4 weeks before the survey was taken. Additionally, this study also examines the impact of extreme weather on self-reported health status and life satisfaction. The SWB variable, life satisfaction, is only available from IFLS 2007 (the $4^{\text {th }}$ wave). The health status and life satisfaction variables are categorized from 1 to 4,1 being very unhealthy or very unsatisfied and 4 being very healthy or very satisfied with the current situation.

\subsubsection{Health insurance in Indonesia}

Understanding the health insurance sector is required if we want to inquire to what extent health insurance participation may, or not, have a role in reducing the impact of weather-related health shock. The social health insurance schemes in Indonesia have started back in the 1960s when they were exclusively offered to civil servants (Askes Asuransi Kesehatan). The services started to develop further only after the 1990s when the government encourages three health insurance schemes, in addition to Askes. The 3 schemes are 1) Jamsostek (Social Insurance for Private Sector Workers), which is mandatory for private employees 2) Private Insurance scheme, which is voluntarily offered by the employer and 3) community health maintenance insurance or known as JPKM-Jaminan Pemeliharaan Kesehatan Masyarakat (Hidayat et al., 2004). In 2005 (which 
later was expanded in 2008), a heavily subsidized health insurance under the Askeskin program (health insurance for the poor) was introduced. This was the first step towards a massive National Social Health Insurance (NSHI) scheme in Indonesia and Sparrow et al. (2013) conclude it has been successful to improve health care services to the poor while those insured who live in urban areas experience an increase in out-of-pocket spending. It may be congruent with our current findings that total health expenditure in Indonesia per person has tripled since 2000 (Mboi et al., 2018).

The Indonesian NSHI scheme or JKN has officially started in 2014 and until November 2017, it enrolled 111 million people in the program (Mboi et al., 2018). National and local government subsidize the premiums of the overall schemes (about 80:20 ratio). The government pays the premiums for the poor and near poor. An ambitious target is set to reach $95 \%$ of the total population by 2019 . According to a recent study, about US $\$ 4 \cdot 8$ billion have been spent to pay 100 million claims for outpatient care and 40 million inpatients (Mboi et al., 2018).

Referring to the statistics of the population sample interviewed in 5 waves of IFLS, participation levels in health insurance schemes steadily increased. Notably, there have been sharp increases in the last 2 rounds compared the first 3 ones. In IFLS 1993, only $10.9 \%$ of adults (age 15 years old and above) reported that they have health insurance. It increases steadily to $12.3 \%$ in IFLS 1997 and then doubled to $27.8 \%$ in IFLS 4 and in the last survey in 2014 jumped to $47.4 \%$ enrollment.

Here, to observe whether having health insurance or not, and different types of insurance matter in reducing weather effects, 3 clusters of enrollment are distinguished: 1 ) without insurance 2) enrolled in a government insurance scheme and 3) enrolled in a private insurance scheme or other types of health insurance. Most of the people who said they have insurance are enrolled in government scheme or later known as NSHI. The proportion of NSHI type with premium subsidy and NSHI without premium subsidy are comparable in the western regions while more dominant for subsidy types in the eastern regions. The fraction of people having private insurance schemes is very small, about 1.5 $\%$ of the total insured sample individuals (except in IFLS 2000 that reached 5\%). More detail information is provided in the descriptive statistics (Table 14). 


\subsubsection{Descriptive Statistics}

Table 14 reports the descriptive statistics of all variables used in the regression models.

Table 14 Summary statistics weather events and individual characteristics

\begin{tabular}{|c|c|c|c|c|c|}
\hline & $\begin{array}{c}\text { IFLS } 1 \\
1993 \\
\end{array}$ & $\begin{array}{c}\text { IFLS } 2 \\
1997 / 1998 \\
\end{array}$ & $\begin{array}{c}\text { IFLS } 3 \\
2000 \\
\end{array}$ & $\begin{array}{c}\text { IFLS } 4 \\
2007 / 2008 \\
\end{array}$ & $\begin{array}{c}\text { IFLS } 5 \\
2014 / 2015 \\
\end{array}$ \\
\hline $\begin{array}{l}\text { Extreme wet months } \\
\text { frequency }(0-3)\end{array}$ & $\begin{array}{c}0.199 \\
(0.461)\end{array}$ & $\begin{array}{l}0.0369 \\
(0.201)\end{array}$ & $\begin{array}{c}0.112 \\
(0.332)\end{array}$ & $\begin{array}{c}0.636 \\
(0.721)\end{array}$ & $\begin{array}{c}0.395 \\
(0.590)\end{array}$ \\
\hline Extreme dry months & 0.191 & 1.605 & 0.204 & 0.0383 & 0.613 \\
\hline frequency $(0-3)$ & $(0.450)$ & $(1.065)$ & $(0.488)$ & $(0.192)$ & $(0.827)$ \\
\hline Extreme hot months & 0.495 & 1.399 & 0.961 & 0.204 & 0.906 \\
\hline frequency (0-3) & $(0.638)$ & $(0.948)$ & $(0.731)$ & $(0.403)$ & $(0.975)$ \\
\hline \multicolumn{6}{|l|}{ Health insurance $(1 / 0)$ : } \\
\hline \multirow[t]{2}{*}{ No insurance } & 0.891 & 0.877 & 0.879 & 0.722 & 0.526 \\
\hline & $(0.312)$ & $(0.328)$ & $(0.326)$ & $(0.448)$ & $(0.499)$ \\
\hline \multirow[t]{2}{*}{ Government scheme } & 0.093 & 0.109 & 0.073 & 0.264 & 0.459 \\
\hline & $(0.291)$ & $(0.312)$ & $(0.260)$ & $(0.441)$ & $(0.498)$ \\
\hline \multirow[t]{2}{*}{ Private insurance } & 0.016 & 0.014 & 0.048 & 0.014 & 0.015 \\
\hline & $(0.125)$ & $(0.118)$ & $(0.213)$ & $(0.118)$ & $(0.121)$ \\
\hline \multirow[t]{2}{*}{ Own a house $(1 / 0)$} & 0.812 & 0.850 & 0.887 & 0.900 & 0.899 \\
\hline & $(0.391)$ & $(0.357)$ & $(0.317)$ & $(0.299)$ & $(0.302)$ \\
\hline \multirow{2}{*}{ Have electricity $(1 / 0)$} & 0.676 & 0.847 & 0.904 & 0.964 & 0.992 \\
\hline & $(0.468)$ & $(0.360)$ & $(0.295)$ & $(0.186)$ & $(0.0864)$ \\
\hline \multirow[t]{2}{*}{ Married dummy $(1 / 0)$} & 0.916 & 0.902 & 0.893 & 0.837 & 0.755 \\
\hline & $(0.278)$ & $(0.297)$ & $(0.309)$ & $(0.370)$ & $(0.430)$ \\
\hline \multirow[t]{2}{*}{ Male dummy $(1 / 0)$} & 0.406 & 0.406 & 0.406 & 0.406 & 0.406 \\
\hline & $(0.491)$ & $(0.491)$ & $(0.491)$ & $(0.491)$ & $(0.491)$ \\
\hline \multirow[t]{2}{*}{ Household size } & 4.895 & 4.843 & 4.743 & 4.269 & 3.999 \\
\hline & $(1.912)$ & $(1.858)$ & $(1.853)$ & $(1.877)$ & $(1.973)$ \\
\hline \multicolumn{6}{|l|}{ Age level (1/0): } \\
\hline \multirow[t]{2}{*}{ Age $15-30$} & 0.300 & 0.151 & 0.078 & 0.008 & 0.000 \\
\hline & $(0.458)$ & $(0.358)$ & $(0.268)$ & $(0.089)$ & $(0.000)$ \\
\hline \multirow[t]{2}{*}{ Age $31-45$} & 0.471 & 0.524 & 0.503 & 0.317 & 0.0952 \\
\hline & $(0.499)$ & $(0.499)$ & $(0.500)$ & $(0.465)$ & $(0.294)$ \\
\hline \multirow[t]{2}{*}{ Age $46-60$} & 0.204 & 0.268 & 0.327 & 0.454 & 0.508 \\
\hline & $(0.403)$ & $(0.443)$ & $(0.469)$ & $(0.498)$ & $(0.500)$ \\
\hline \multirow[t]{2}{*}{ Age $60+$} & 0.0247 & 0.0573 & 0.0925 & 0.221 & 0.397 \\
\hline & $(0.155)$ & $(0.232)$ & $(0.290)$ & $(0.415)$ & $(0.489)$ \\
\hline \multicolumn{6}{|l|}{ Education level (1/0): } \\
\hline \multirow[t]{2}{*}{ No schooling } & 0.195 & 0.196 & 0.201 & 0.202 & 0.200 \\
\hline & $(0.397)$ & $(0.397)$ & $(0.401)$ & $(0.401)$ & $(0.400)$ \\
\hline Elementary & 0.536 & 0.536 & 0.538 & 0.536 & 0.533 \\
\hline (1-6 year) & $(0.499)$ & $(0.499)$ & $(0.499)$ & $(0.499)$ & $(0.499)$ \\
\hline Junior-Senior high & 0.239 & 0.232 & 0.223 & 0.215 & 0.217 \\
\hline (7-12 year) & $(0.426)$ & $(0.420)$ & $(0.416)$ & $(0.411)$ & $(0.412)$ \\
\hline University & 0.0297 & 0.0185 & 0.0383 & 0.0473 & 0.0499 \\
\hline$(+12$ year $)$ & $(0.170)$ & $(0.135)$ & $(0.192)$ & $(0.212)$ & $(0.218)$ \\
\hline \multirow[t]{2}{*}{ Total Assets HH (US\$) } & $2,764.67$ & $2,364.56$ & $4,249.80$ & $7,428.45$ & $11,320.57$ \\
\hline & $(17,641.22)$ & $(10,822.96)$ & $(9,833.31)$ & $(14,702.38)$ & $(22,596.33)$ \\
\hline \multirow[t]{2}{*}{ Farm income HH (US\$) } & 29.01 & 28.88 & 67.93 & 118.10 & 175.54 \\
\hline & (227.09) & $(86.99)$ & $(272.83)$ & $(334.26)$ & $(434.35)$ \\
\hline \multirow[t]{2}{*}{ Monthly expenditure (US\$) } & 39.38 & 59.32 & 53.17 & 63.34 & 91.03 \\
\hline & $(42.83)$ & $(159.42)$ & (77.41) & (57.28) & $(80.90)$ \\
\hline \multicolumn{6}{|l|}{ Symptoms of illness: } \\
\hline Mild illness $(1 / 0)$ & 0.566 & 0.693 & 0.712 & 0.675 & 0.741 \\
\hline & $(0.496)$ & $(0.461)$ & $(0.453)$ & $(0.468)$ & $(0.438)$ \\
\hline Digestive illness $(1 / 0)$ & 0.0336 & 0.206 & 0.206 & 0.184 & 0.277 \\
\hline & $(0.180)$ & $(0.405)$ & $(0.405)$ & $(0.387)$ & $(0.448)$ \\
\hline
\end{tabular}




\begin{tabular}{|c|c|c|c|c|c|}
\hline Respiratory illness $(1 / 0)$ & $\begin{array}{c}0.0369 \\
(0.189)\end{array}$ & $\begin{array}{c}0.388 \\
(0.487)\end{array}$ & $\begin{array}{c}0.0693 \\
(0.254)\end{array}$ & $\begin{array}{c}0.0648 \\
(0.246)\end{array}$ & $\begin{array}{c}0.102 \\
(0.303)\end{array}$ \\
\hline \multirow[t]{2}{*}{ Minor infections $(1 / 0)$} & 0.0456 & 0.132 & 0.158 & 0.141 & 0.233 \\
\hline & $(0.209)$ & $(0.339)$ & $(0.365)$ & $(0.348)$ & $(0.422)$ \\
\hline \multirow[t]{2}{*}{ Outpatient cost } & 1.21 & 1.11 & 0.92 & 0.95 & 1.25 \\
\hline & $(20.03)$ & $(13.69)$ & $(5.83)$ & $(6.64)$ & $(7.27)$ \\
\hline \multirow[t]{2}{*}{ Bedrest } & (2) & 0.337 & 0.325 & 0.319 & 0.849 \\
\hline & (.) & $(1.701)$ & $(1.728)$ & $(1.724)$ & $(3.513)$ \\
\hline \multirow[t]{2}{*}{ Outpatient visit } & 0.253 & 0.234 & 0.220 & 0.251 & 0.393 \\
\hline & $(0.697)$ & $(0.646)$ & $(0.620)$ & $(0.905)$ & (1.104) \\
\hline \multirow[t]{2}{*}{ Health status ( 1 to 4 ) } & 3.068 & 2.986 & 2.952 & 2.910 & 2.776 \\
\hline & $(0.513)$ & $(0.440)$ & $(0.437)$ & $(0.505)$ & $(0.731)$ \\
\hline \multirow[t]{2}{*}{ Life satisfaction (1 to 4 ) } & 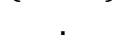 & . & 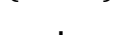 & 2.930 & 2.938 \\
\hline & (.) & (.) & (.) & (0.397) & $(0.499)$ \\
\hline
\end{tabular}

Note: the mean coefficient statistics are calculated from 5 waves of the Indonesian Family Life Survey; standard deviations are in parentheses. Variables with monetary units (incomes, assets, monthly expenditures and outpatient cost) are inflation adjusted at 2010 price. The original values in Indonesian rupiah (IDR) are converted into US Dollar, based on Indonesian Central Bank yearly average in 2010: https://finance.detik.com/bursa-dan-valas/d1536232/rupiah-menguat-44-selama-2010 (1 USD= IDR 9,081). These variables, except outpatient cost, are measured at a household level per capita. Outcome variables illness symptoms, outpatient cost, bedrest, outpatient visit are measured in the last 4 weeks. Outcome variables health status and life satisfaction indicate present own-observation of the individual at the time of the survey. The values range from 1 to 4 , which indicate 1 being very unhealthy or very unsatisfied, 2 is unhealthy and unsatisfied, 3 is healthy or satisfied and 4 is very healthy or very unsatisfied.

\subsection{Findings}

\subsubsection{Marginal effects of extreme weather and symptoms of illness}

The relationship between extreme rainfall and symptom of illness is examined using probit regression. Accordingly, Table 15 reports the marginal effects of experiencing wet and dry months in the three months observation on self-reported symptoms. The estimated coefficients are the predicted probability of changes in outcomes when the frequency of extreme rainfall increases by 1 month, holding other predictors constant at their means. The results suggest that dry months are more associated with illness symptoms than wet month episodes. Indications that drought matters more for healthrelated outcomes of an individual than experiencing excessive rains may be related to weather effects on the agricultural sector. Findings of Lohmann and Lechtenfeld (2015) in Vietnam suggest that rural households exposed to drought measured in the last 3 months have a higher probability to be affected by the disease, particularly when there are negative impacts of drought on the agricultural outputs.

For the purpose of this paper, information from self-reported health problems in IFLS is grouped into 4 symptoms: mild, digestive, respiratory and infectious. The first includes symptoms such as a headache, runny nose, sore eyes, cough, cold, or nausea. The regression results show that there is a statistically significant positive relationship between the variations in experiencing dry months and changes in mild symptoms (Table 
15, column mild and row Extreme dry month frequency). The marginal effect is 0.01 , which means the expected difference in probability of having mild symptoms associated with a one month difference in extreme dry month frequency is a 1 percentage point (pp) decrease. It appears that experiencing wet month(s) is not associated with having mild symptoms (as the result is not statistically significant).

There is statistically significant evidence that the more often rainfall-related shocks occurred, the greater the probability of having digestive-tract-related problems. Findings in Table 15 (column digestive) suggest that when the frequency of wet months increases by 1 unit ( 1 month with excessive rainfall), the predicted probability of having digestive problem increases by $1.4 \mathrm{pp}$. On the other hand, dry months are also partially associated with the likelihood of having digestive symptoms. For each one dry month difference, the average marginal effect on probability to have a digestive problem increased by $0.7 \mathrm{pp}$. For respiratory problems, the estimated results suggest a statistically significant association with dry month episodes. An additional one dry month leads to a $1.3 \mathrm{pp}$ rise in the probability of experiencing a respiratory problem ${ }^{37}$.

Table 15 Average marginal effects on symptoms of illness

\begin{tabular}{lcccc}
\hline & \multicolumn{3}{c}{ Dependent Variable: symptoms of illness } \\
\cline { 2 - 5 } & mild & digestive & respiratory & infectious \\
\hline Extreme wet month frequency (0-3) & -0.004 & $0.014^{* * *}$ & -0.003 & -0.005 \\
Extreme dry month frequency (0-3) & $(0.006)$ & $(0.004)$ & $(0.005)$ & $(0.005)$ \\
& $0.010^{* *}$ & $0.007^{* *}$ & $0.013^{* * *}$ & 0.002 \\
Extreme hot month frequency (0-3) & $(0.004)$ & $(0.003)$ & $(0.003)$ & $(0.003)$ \\
Age & -0.005 & 0.003 & -0.001 & -0.005 \\
Household size & $(0.004)$ & $(0.003)$ & $(0.003)$ & $(0.003)$ \\
Years of schooling & $-0.001^{*}$ & $-0.001^{* *}$ & $0.002^{* * *}$ & $0.003^{* * *}$ \\
& $(0.000)$ & $(0.000)$ & $(0.000)$ & $(0.000)$ \\
Male (1/0) & $0.005^{* * *}$ & 0.002 & 0.001 & -0.001 \\
& $(0.002)$ & $(0.001)$ & $(0.001)$ & $(0.001)$ \\
& $0.003^{* * *}$ & 0.000 & $-0.004^{* * *}$ & $-0.002^{* * *}$
\end{tabular}

37 Historically, many areas in Indonesia experienced very extreme dry months in 1997-1998 which triggered massive forest fires and harvest failures. The drought's effects are partially worsened by the severe El Nino as well as 1997/1998 economic crisis, which may also affect the health conditions of individuals, which in the case may be related to a respiratory problem (see, for example, Kim et al. (2017) that examine the health effect of air pollution due to forest fire). To account for potential bias resulted from these time-specific events, the empirical analysis has controlled the time fixed-effects (the estimated partial effect without wave-effect control would have been statistically significant 7.2 pp. at 99 confidence level as opposed to $1.3 \mathrm{pp}$ with time-fixed-effects). 


\begin{tabular}{lcccc} 
& $(0.007)$ & $(0.005)$ & $(0.005)$ & $(0.006)$ \\
Married (1/0) & $-0.033^{* * *}$ & -0.009 & -0.007 & -0.003 \\
Own house & $(0.010)$ & $(0.007)$ & $(0.007)$ & $(0.008)$ \\
& $-0.036^{* * *}$ & -0.010 & -0.001 & -0.006 \\
Have electricity & $(0.009)$ & $(0.007)$ & $(0.007)$ & $(0.008)$ \\
& 0.013 & -0.008 & 0.002 & $-0.030^{* * *}$ \\
Government health insurance $(1 / 0)$ & $(0.010)$ & $(0.008)$ & $(0.008)$ & $(0.010)$ \\
& 0.008 & $0.013^{* *}$ & 0.007 & $0.012^{*}$ \\
Private health insurance $(1 / 0)$ & $(0.008)$ & $(0.006)$ & $(0.007)$ & $(0.007)$ \\
& 0.009 & 0.009 & -0.014 & -0.022 \\
Monthly expenditure & $(0.019)$ & $(0.015)$ & $(0.015)$ & $(0.016)$ \\
(100 USD) & 0.010 & $0.004^{*}$ & 0.003 & $0.004^{*}$ \\
Total Assets per capita last survey & $(0.006)$ & $(0.002)$ & $(0.002)$ & $(0.003)$ \\
(100 USD) & 0.000 & 0.000 & -0.006 & $-0.044^{* *}$ \\
Farm income per capita last survey & $(0.000)$ & $(0.000)$ & $(0.018)$ & $(0.022)$ \\
(100 USD) & -0.002 & -0.001 & 0.001 & -0.000 \\
$N$ & $(0.001)$ & $(0.001)$ & $(0.001)$ & $(0.001)$ \\
\hline
\end{tabular}

Note: Standard errors in parentheses, clustered at sub-district. ${ }^{*} p<0.10,{ }^{* *} p<0.05,{ }^{* * *} p<0.01$. Model specifications account for individual and wave fixed-effect using probit regression. Outcomes are dichotomous (dummy) variables illness symptoms in the last 4 weeks and the predicted results are marginal effects. Mild illness symptoms include a headache, runny nose, sore eye, cough, cold, and nausea. Symptoms of digestive problems include stomach ache and diarrhea. Symptoms of respiratory problems include difficult breathing. Symptoms of infectious illness include skin infection/scabies/ulcers and eye infection. Extreme wet month frequency is the total number of the month when actual monthly precipitation exceeds the 50 years historic extreme upper threshold (p90) of cumulative rainfall in a month. Extreme wet month frequency is the total number of the month when monthly precipitation below the 50 years historic extreme lower threshold (p10) of cumulative rainfall in a month.

Last, findings in Table 15 does not show significant estimates on the influence of wet month frequency over the probability of having respiratory problems nor there are significant associations between rainfall-related conditions and probability of having symptoms of infectious illness, such as skin infection/scabies/ulcers and eye infection. The variable measuring the frequency of months with extremely hot temperature does not show any estimated partial effects on all the health outcomes being considered. Overall, the findings suggest that there is a robust link between extreme rainfall events and the probability of having digestive problems. However, partial effects on other illness symptoms are less clear.

\subsubsection{Extreme rainfall effects on other health-related outcomes}

Table 16 reports predicted changes in several health-related outcomes due to experiencing extreme weather episodes. The outcomes of interest being examined are outpatient expenditures, stay in bed due to illness (bedrest), a number of doctor/ medical facility visits for outpatient care and self-reported health status. The first three dependent variables indicate realizations in the last 4 weeks while the health status 
refers to a self-assessment condition in general. According to the results, dry months are significantly associated with two observable outcomes: bed-rest and outpatient visits. The findings, however, do not show any statistically significant results that indicate extreme rainfall episodes in the last 3 months have an effect on outpatient costs and selfreported health status. The insignificance results in individual's outpatient expenditure may be reflections of adaptation or mitigating strategies, such as for example, health insurance.

Table 16 Health-related outcome impacts due to extreme weather

\begin{tabular}{|c|c|c|c|c|}
\hline & \multicolumn{4}{|c|}{ Dependent Variable: health-related outcomes } \\
\hline & $\begin{array}{l}\text { Outpatient } \\
\text { cost (USD) }\end{array}$ & $\begin{array}{c}\text { Bedrest } \\
\text { (days) }\end{array}$ & $\begin{array}{l}\text { Outpatient } \\
\text { visit (\#) }\end{array}$ & $\begin{array}{c}\text { Health } \\
\text { status }(1-4)\end{array}$ \\
\hline Extreme wet month frequency $(0-$ & $\begin{array}{l}-0.897 \\
(0.666)\end{array}$ & $\begin{array}{c}0.357 \\
(0.258)\end{array}$ & $\begin{array}{c}-0.030 \\
(0.049)\end{array}$ & $\begin{array}{c}0.006 \\
(0.015)\end{array}$ \\
\hline Extreme dry month frequency $(0-3)$ & $\begin{array}{c}0.027 \\
(0.524)\end{array}$ & $\begin{array}{l}0.437^{* *} \\
(0.179)\end{array}$ & $\begin{array}{c}0.066^{*} \\
(0.036)\end{array}$ & $\begin{array}{c}0.001 \\
(0.010)\end{array}$ \\
\hline Extreme hot month frequency $(0-3)$ & $\begin{array}{l}-0.106 \\
(0.432)\end{array}$ & $\begin{array}{l}-0.307^{*} \\
(0.164)\end{array}$ & $\begin{array}{l}-0.076^{* *} \\
(0.033)\end{array}$ & $\begin{array}{l}0.040^{* * *} \\
(0.009)\end{array}$ \\
\hline Age & $\begin{array}{c}0.037 \\
(0.040)\end{array}$ & $\begin{array}{l}0.122^{* * *} \\
(0.017)\end{array}$ & $\begin{array}{l}0.017^{* * *} \\
(0.003)\end{array}$ & $\begin{array}{l}-0.016^{* * *} \\
(0.001)\end{array}$ \\
\hline Household size & $\begin{array}{c}0.106 \\
(0.184)\end{array}$ & $\begin{array}{l}0.198^{* * *} \\
(0.072)\end{array}$ & $\begin{array}{c}0.016 \\
(0.015)\end{array}$ & $\begin{array}{l}-0.012^{* * *} \\
(0.004)\end{array}$ \\
\hline Years of schooling & $\begin{array}{l}0.423^{* * *} \\
(0.106)\end{array}$ & $\begin{array}{l}-0.245^{* * *} \\
(0.039)\end{array}$ & $\begin{array}{l}0.036^{* * *} \\
(0.008)\end{array}$ & $\begin{array}{c}0.002 \\
(0.002)\end{array}$ \\
\hline Male $(1 / 0)$ & $\begin{array}{l}-7.814^{* * *} \\
(1.334)\end{array}$ & $\begin{array}{l}-1.060^{* * *} \\
(0.317)\end{array}$ & $\begin{array}{l}-0.788^{* * *} \\
(0.065)\end{array}$ & $\begin{array}{l}0.104^{* * *} \\
(0.018)\end{array}$ \\
\hline Married (1/0) & $\begin{array}{c}1.715 \\
(1.176)\end{array}$ & $\begin{array}{l}-1.191^{* * *} \\
(0.404)\end{array}$ & $\begin{array}{c}0.124 \\
(0.080)\end{array}$ & $\begin{array}{l}0.077^{* * *} \\
(0.025)\end{array}$ \\
\hline Own house & $\begin{array}{l}-0.388 \\
(1.230)\end{array}$ & $\begin{array}{l}-0.852^{*} \\
(0.439)\end{array}$ & $\begin{array}{c}0.007 \\
(0.082)\end{array}$ & $\begin{array}{l}0.066^{* * *} \\
(0.022)\end{array}$ \\
\hline Have electricity & $\begin{array}{l}5.969^{* * *} \\
(1.438)\end{array}$ & $\begin{array}{l}-1.513^{* * *} \\
(0.522)\end{array}$ & $\begin{array}{l}0.380^{* * *} \\
(0.095)\end{array}$ & $\begin{array}{l}0.107^{* * *} \\
(0.023)\end{array}$ \\
\hline Government health insurance $(1 / 0)$ & $\begin{array}{l}-1.836^{*} \\
(1.033)\end{array}$ & $\begin{array}{c}0.618^{*} \\
(0.345)\end{array}$ & $\begin{array}{l}0.512^{* * *} \\
(0.070)\end{array}$ & $\begin{array}{c}0.006 \\
(0.022)\end{array}$ \\
\hline Private health insurance $(1 / 0)$ & $\begin{array}{l}4.265^{* *} \\
(1.979)\end{array}$ & $\begin{array}{c}0.190 \\
(0.929)\end{array}$ & $\begin{array}{l}0.817^{* * *} \\
(0.148)\end{array}$ & $\begin{array}{c}0.011 \\
(0.045)\end{array}$ \\
\hline $\begin{array}{l}\text { Monthly expenditure } \\
\text { (100 USD) }\end{array}$ & $\begin{array}{l}1.550^{* * *} \\
(0.516)\end{array}$ & $\begin{array}{c}0.107 \\
(0.100)\end{array}$ & $\begin{array}{l}0.113^{* * *} \\
(0.038)\end{array}$ & $\begin{array}{l}-0.018^{* *} \\
(0.008)\end{array}$ \\
\hline $\begin{array}{l}\text { Total Assets per capita last survey } \\
\text { (100 USD) }\end{array}$ & $\begin{array}{l}0.005^{* * *} \\
(0.002)\end{array}$ & $\begin{array}{c}0.000 \\
(0.001)\end{array}$ & $\begin{array}{l}0.000^{* * *} \\
(0.000)\end{array}$ & $\begin{array}{c}0.000 \\
(0.000)\end{array}$ \\
\hline $\begin{array}{l}\text { Farm income per capita last survey } \\
\text { (100 USD) }\end{array}$ & $\begin{array}{c}0.127 \\
(0.155)\end{array}$ & $\begin{array}{l}-0.087^{*} \\
(0.045)\end{array}$ & $\begin{array}{l}-0.007 \\
(0.009)\end{array}$ & $\begin{array}{c}0.001 \\
(0.003)\end{array}$ \\
\hline$N$ & 29,930 & 29,930 & 29,930 & 29,930 \\
\hline
\end{tabular}

Note: Clustered at sub-district standard errors in parentheses. ${ }^{*} p<0.10,{ }^{* *} p<0.05,{ }^{* * *} p<0.01$. Model specifications account for individual and wave fixed-effect using tobit (cost, bedrest, and visit) and ordered 
probit regression (health status). Dependent variables include outpatient cost, bedrest and visit for outpatient care in the last 4 weeks as well as current self-reported heath status (" 1 " unhealthy to " 4 " very healthy). Extreme wet month frequency is the total number of the month when actual monthly precipitation exceeds the 50 years historic extreme upper threshold (p90) of cumulative rainfall in a month. Extreme dry month frequency is the total number of the month when monthly precipitation below the 50 years historic extreme lower threshold ( $\mathrm{p} 10)$ of monthly cumulative rainfall.

Referring to Table 16, there is a statistically significant correlation between dry month conditions and bedrest that measures a number of days staying bed-ridden because of illness. The estimated rainfall effect equals to an expectation of a 0.437-day increase in bedrest when dry month occurrence increase by 1 unit $(1$ month with rainfall exceeding the upper extreme threshold) - holding other variables in the model constant. Or equally, it suggests that if weather conditions in the last 3 months are all extremely dry, an adult individual would, on average, be bed-ridden for an additional 1.31 days. Individual characteristics are controlled for in the model specifications, and many of them signal robust relationships with the outcome of interest. For example, age, gender, and household size are positively correlated with illness bedrest (significance at a 99\% confidence level).

The magnitude of extreme weather impact on the outpatient visit, although the coefficient is statistically significant, is very small. The estimates indicate that 1 dry month increase is associated with an additional 0.066-time visit for outpatient care. The small effect of dry month and insignificance result of wet month suggest that adverse weather conditions may not the influential factor for the outpatient visit. If we look at the control variables' coefficients, individual factors such as age, education level, having health insurance, are those that significantly affect the number of outpatient visit.

\subsubsection{Adverse weather conditions and subjective well-being}

Next, we quantify the effects of extreme weather on life satisfaction using ordered probit regression. Table 17 reports the ordered prob-odds (probit) regression coefficients (not the marginal effect). The column IFLS 2007 is for cross-section (CS) using the 2007 survey (and same for IFLS 2014). The column pooled CS is for pooled cross-section model using 2007 and 2014 survey and panel 07-14 column is for panel model (recall that life satisfaction data are available in the last 2 waves of IFLS). The results suggest significant negative correlations between extreme rainfall conditions and life satisfaction are observable in all models, suggesting a negative impact on life satisfaction after experiencing wet and dry month. In panel model, for example, if an individual were to 
experience an additional wet month by one month, his ordered log-odds of being in higher life satisfaction category would drop by 0.03 while the other variables are held constant.

Table 17 Life satisfaction impacts due to extreme weather

\begin{tabular}{|c|c|c|c|c|}
\hline & \multicolumn{4}{|c|}{ Dependent Variable: Life Satisfaction } \\
\hline & IFLS 2007 & IFLS 2014 & Pooled CS & Panel 07-14 \\
\hline Extreme wet month frequency $(0-3)$ & $\begin{array}{l}-0.021^{*} \\
(0.012)\end{array}$ & $\begin{array}{l}-0.037^{* *} \\
(0.016)\end{array}$ & $\begin{array}{l}-0.021^{* *} \\
(0.010)\end{array}$ & $\begin{array}{l}-0.032^{* * *} \\
(0.011)\end{array}$ \\
\hline Extreme dry month frequency $(0-3)$ & $\begin{array}{l}-0.088^{* *} \\
(0.039)\end{array}$ & $\begin{array}{l}-0.016 \\
(0.011)\end{array}$ & $\begin{array}{l}-0.023^{* *} \\
(0.010)\end{array}$ & $\begin{array}{l}-0.010 \\
(0.011)\end{array}$ \\
\hline Extreme hot month frequency $(0-3)$ & $\begin{array}{l}0.098^{* * *} \\
(0.020)\end{array}$ & $\begin{array}{l}-0.008 \\
(0.010)\end{array}$ & $\begin{array}{l}0.032^{* * *} \\
(0.009)\end{array}$ & $\begin{array}{l}0.019^{* *} \\
(0.010)\end{array}$ \\
\hline Age & $\begin{array}{l}-0.008^{* * *} \\
(0.001)\end{array}$ & $\begin{array}{l}-0.009^{* * *} \\
(0.001)\end{array}$ & $\begin{array}{l}-0.009^{* * *} \\
(0.000)\end{array}$ & $\begin{array}{l}-0.009^{* * *} \\
(0.000)\end{array}$ \\
\hline Household size & $\begin{array}{l}-0.005 \\
(0.005)\end{array}$ & $\begin{array}{c}0.007 \\
(0.004)\end{array}$ & $\begin{array}{c}0.003 \\
(0.003)\end{array}$ & $\begin{array}{c}0.003 \\
(0.003)\end{array}$ \\
\hline Years of schooling & $\begin{array}{l}0.030^{* * *} \\
(0.002)\end{array}$ & $\begin{array}{l}0.029^{* * *} \\
(0.002)\end{array}$ & $\begin{array}{l}0.030^{* * *} \\
(0.002)\end{array}$ & $\begin{array}{l}0.030^{* * *} \\
(0.002)\end{array}$ \\
\hline Male $(1 / 0)$ & $\begin{array}{l}-0.098^{* * *} \\
(0.017)\end{array}$ & $\begin{array}{l}-0.051^{* * *} \\
(0.014)\end{array}$ & $\begin{array}{l}-0.071^{* * *} \\
(0.011)\end{array}$ & $\begin{array}{l}-0.075^{* * *} \\
(0.011)\end{array}$ \\
\hline Married (1/0) & $\begin{array}{l}0.284^{* * *} \\
(0.019)\end{array}$ & $\begin{array}{l}0.250^{* * *} \\
(0.016)\end{array}$ & $\begin{array}{l}0.267^{* * *} \\
(0.012)\end{array}$ & $\begin{array}{l}0.258^{* * *} \\
(0.013)\end{array}$ \\
\hline Own house & $\begin{array}{c}0.040^{*} \\
(0.021)\end{array}$ & $\begin{array}{l}0.080^{* * *} \\
(0.018)\end{array}$ & $\begin{array}{l}0.051^{* * *} \\
(0.014)\end{array}$ & $\begin{array}{l}0.042^{* * *} \\
(0.014)\end{array}$ \\
\hline Have electricity & $\begin{array}{l}0.162^{* * *} \\
(0.048)\end{array}$ & $\begin{array}{l}0.346^{* * *} \\
(0.086)\end{array}$ & $\begin{array}{l}0.209^{* * *} \\
(0.040)\end{array}$ & $\begin{array}{l}0.190^{* * *} \\
(0.041)\end{array}$ \\
\hline Government health insurance $(1 / 0)$ & $\begin{array}{l}0.050^{* *} \\
(0.020)\end{array}$ & $\begin{array}{c}0.025^{*} \\
(0.015)\end{array}$ & $\begin{array}{l}0.036^{* * *} \\
(0.012)\end{array}$ & $\begin{array}{l}0.041^{* * *} \\
(0.013)\end{array}$ \\
\hline Private health insurance $(1 / 0)$ & $\begin{array}{l}0.206^{* * *} \\
(0.050)\end{array}$ & $\begin{array}{l}0.211^{* * *} \\
(0.042)\end{array}$ & $\begin{array}{l}0.221^{* * *} \\
(0.032)\end{array}$ & $\begin{array}{l}0.212^{* * *} \\
(0.033)\end{array}$ \\
\hline $\begin{array}{l}\text { Monthly expenditure } \\
\text { (100 USD) }\end{array}$ & $\begin{array}{l}0.131^{* * *} \\
(0.015)\end{array}$ & $\begin{array}{l}0.069^{* * *} \\
(0.010)\end{array}$ & $\begin{array}{l}0.092^{* * *} \\
(0.009)\end{array}$ & $\begin{array}{l}0.092^{* * *} \\
(0.009)\end{array}$ \\
\hline $\begin{array}{l}\text { Total Assets per capita last survey } \\
\text { (100 USD) }\end{array}$ & $\begin{array}{l}0.000^{* * *} \\
(0.000)\end{array}$ & $\begin{array}{l}0.000^{* * *} \\
(0.000)\end{array}$ & $\begin{array}{l}0.000^{* * *} \\
(0.000)\end{array}$ & $\begin{array}{l}0.000^{* * *} \\
(0.000)\end{array}$ \\
\hline Farm income per capita last survey & $\begin{array}{l}0.004^{* * *} \\
(0.001)\end{array}$ & $\begin{array}{l}0.004^{* * *} \\
(0.001)\end{array}$ & $\begin{array}{l}0.003^{* * *} \\
(0.001)\end{array}$ & $\begin{array}{l}0.004^{* * *} \\
(0.001)\end{array}$ \\
\hline Individual fixed-effect & NO & NO & NO & YES \\
\hline Sub-district fixed-effect & YES & YES & YES & YES \\
\hline Wave fixed-effect & NO & NO & YES & YES \\
\hline$N$ & 28,610 & 33,394 & 62,178 & 57,220 \\
\hline
\end{tabular}

Note: Clustered at sub-district standard errors in parentheses. ${ }^{*} p<0.10,{ }^{* *} p<0.05,{ }^{* * *} p<0.01$. Model specifications use ordered probit regression accounting for individual and time fixed-effects. Life satisfaction has a value of "1" unhappy to "4" very happy. Extreme wet month frequency is the total number of the month when actual monthly precipitation exceeds the 50 years historic extreme upper threshold (p90) of cumulative rainfall in a month. Extreme dry month frequency is the total number of the month when monthly precipitation below the 50 years historic extreme lower threshold (p10) of monthly cumulative rainfall. 
The effect on the odds of getting a higher or lower value of life satisfaction is robust to varying model specifications, but the panel model is the relevant one. The results suggest that wet months are more associated with predicted changes in life satisfaction than dry month episodes. The empirical specifications consider socioeconomic, including demographic, control variable as well as sub-district fixed-effects in CS and pooled CS models, and obviously individual fixed-effect in panel model (as panel entity is individual), to account for time-invariant characteristics in the drought-SWB nexus.

\subsubsection{Individual and household coping strategies to reduce impact}

Next, we attempt to identify risk mitigating strategies that an individual, or household, takes to reduce the consequence of extreme weather impacts on health outcomes. This section focuses on the impact of having formal and informal insurance mechanisms. The negative sign of the coefficients indicates the role of these factors meets the expectation of reducing weather impacts.

\subsubsection{Reviews on all coping strategies}

Table 18 reports the effects of coping strategies, all considered, in reducing extreme weather impacts on health-related outcomes. The findings show mixed results between the effects of coping factors, where it appears they are more effective to lessen the impact during dry months than wet months. For example, having insurance does not have an effect that reduces the impact of wet months on outpatient expenditure, but it does have a mitigating effect during dry month episodes. In the first column (cost) and interaction effect's row (Extreme dry X private health insurance), it shows that an individual enrolled in a private insurance scheme would experience 81 cents US dollar less impact on his/her outpatient spending in one month than someone without insurance.

Table 18 The vulnerability of weather effects on health-related outcomes

\begin{tabular}{lccc}
\hline & \multicolumn{3}{c}{ Dependent Variable: health-related outcomes } \\
\cline { 2 - 4 } & cost & bedrest & visit \\
\hline Extreme wet months frequency $(0-3)$ & $-0.2541^{* *}$ & 0.1306 & $-0.1516^{* *}$ \\
Extreme dry months frequency $(0-3)$ & $(0.1235)$ & $(0.3962)$ & $(0.0770)$ \\
& -0.1189 & $0.6539^{* * *}$ & $0.1113^{* *}$ \\
Government health insurance & $(0.1358)$ & $(0.2186)$ & $(0.0457)$ \\
Private health insurance & 0.1727 & $0.9530^{*}$ & $0.4786^{* * *}$ \\
& $(0.2764)$ & $(0.4892)$ & $(0.0928)$ \\
HH monthly expenditure (HH welfare) & 0.1965 & -0.2699 & $0.7465^{* * *}$ \\
& $(0.2261)$ & $(1.3063)$ & $(0.1874)$ \\
& $0.1295^{* * *}$ & 0.2807 & $0.2371^{* * *}$
\end{tabular}


HH Total Assets per capita

HH Farm Income per capita

Extreme wet X govt. health insurance

Extreme wet X private health insurance

Extreme wet X HH monthly expenditure

Extreme wet X HH Total Assets per capita

Extreme wet X HH Farm Income per capita

Extreme dry X govt. health insurance

Extreme dry X private health insurance

Extreme dry X HH monthly expenditure

Extreme dry X HH Total Assets per capita

Extreme dry X HH Farm Income per capita

Extreme hot month frequency (0-3)

Age

Years of schooling

Household size

Male (1/0)

Married (1/0)

Own house

Have electricity
$(0.0502)$

$0.0005^{* * *}$

(0.0001)

0.0055

(0.0261)

$-0.3074$

(0.2495)

$-0.2782$

(0.3216)

$0.1377^{* *}$

(0.0560)

$-0.0004^{*}$

(0.0002)

0.0031

(0.0113)

$-0.1545$

(0.1679)

$-0.8114^{* * *}$

(0.2868)

$-0.0055$

$(0.0231)$

$-0.0001^{*}$

(0.0001)

$-0.0063$

(0.0177)

0.0171

(0.0774)

$-0.0096$

(0.0089)

$0.0271^{* *}$

(0.0135)

$-0.0066$

(0.0242)

0.2521

(0.1690)

$-0.0984$

(0.2473)

$-0.5013^{* *}$

(0.2157)

0.5202

(0.4216)
(0.1903)

$-0.0037^{*}$

(0.0022)

0.1155

(0.0777)

$-0.0223$

(0.5587)

2.6263*

(1.4890)

0.1978

(0.3507)

0.0029

(0.0024)

$-0.1272$

(0.0891)

$-0.4958$

(0.3816)

$-0.6939$

(1.1875)

$-0.1125$

(0.0968)

0.0008

(0.0010)

$-0.0739$

(0.0638)

$-0.2742^{*}$

(0.1645)

$0.1245^{* * *}$

(0.0169)

$-0.2415^{* * *}$

(0.0385)

$0.2198^{* * *}$

(0.0706)

$-1.0699^{* * *}$

(0.3165)

$-1.2345^{* * *}$

(0.4038)

$-0.8526^{*}$

(0.4395)

$-1.4483^{* * *}$

(0.5219)
(0.0739)

$0.0004^{* *}$

(0.0002)

$-0.0238$

(0.0150)

0.0243

(0.1103)

0.0105

(0.3068)

$0.1666^{* *}$

(0.0754)

$-0.0004$

(0.0003)

0.0221

(0.0135)

0.0527

(0.0734)

0.1570

(0.2091)

$-0.0960^{* * *}$

(0.0340)

$-0.0002$

(0.0002)

$0.0307^{\text {** }}$

(0.0127)

$-0.0821^{* *}$

(0.0329)

$0.0173^{* * *}$

(0.0032)

$0.0323^{* * *}$

(0.0077)

0.0218

(0.0151)

$-0.7839^{* * *}$

(0.0645)

0.1220

(0.0798)

0.0153

(0.0813)

$0.3585^{\text {*** }}$

(0.0951)

29,930

29,930

29,930

Note: Clustered at sub-district standard errors in parentheses. ${ }^{*} \mathrm{p}<0.10,{ }^{* *} \mathrm{p}<0.05,{ }^{* * *} \mathrm{p}<0.01$. Model specifications account for individual and wave fixed-effect. Dependent variables include outpatient cost, bedrest and visit for outpatient care in the last 4 weeks as well as current self-reported health status (" 1 " unhealthy to "4" very healthy). Interaction effects are reported in rows with interacting signs, for example, extreme wet X govt. health insurance and Extreme dry X private health insurance. Extreme wet month frequency is the total number of the month when actual monthly precipitation exceeds the 50 years historic extreme upper threshold (p90) of cumulative rainfall in a month. Extreme dry month frequency is the total number of the month when monthly precipitation below the 50 years historic extreme lower threshold (p10) of monthly cumulative rainfall. 
Another coping factor that seems to be effectively reducing the effect of dry month episodes is the economic condition (based on household monthly expenditure). An individual from a household that has high monthly expenditure (thus considered as coming from a good economic background) seems to experience less effect on the outpatient visit ( 0.1 fewer times). Perhaps when someone is better off in terms of economic condition, he or she has more capability to do adaptation measures during a dry month, such as buying necessary medicines when feeling unwell without having to go to a medical facility for an outpatient care. Another way to interpret this economic condition effect is through a marginal effect plot. First, we calculate the values of margins of the interacted regression (dry month X economic condition). The results in Table 19 show how much changes in an outpatient visit with a one additional dry month, holding monthly expenditure constant at different values(here we calculate at two extreme values 100 dollars and 8000 dollars). The estimated coefficients are thus simple slopes. The results in Table 19 suggest that the simple slopes for the coping characteristic economic condition (continuous variable economic condition) are significant when there was only one month of dry months (see insignificant results for the dry month $>=2$ in row 7 and 8, column margin and z-score). Next, we plot these simple slopes for each of the values of monthly expenditure (economic condition) - see Figure 10.

Table 19 Marginal effect of the economic condition against dry month episodes

Expression: Linear prediction, predict(0)

$\begin{array}{rlr}\text { 1._at: exp100_usd } & = & 1 \\ \text { tot_dry } & = & 0 \\ \text { 2._at: exp100_usd } & = & 1 \\ \text { tot_dry } & = & 1 \\ \text { 3._at: exp100_usd } & = & 1 \\ \text { tot_dry } & = & 2 \\ \text { 4_at: exp100_usd } & = & 1 \\ \text { tot_dry } & = & 3 \\ \text { 5._at: exp100_usd } & = & 80 \\ \text { tot_dry } & = & 0 \\ \text { 6._at: exp100_usd } & = & 80 \\ \text { tot_dry } & = & 1 \\ \text { 7._at: exp100_usd } & = & 80 \\ \text { tot_dry } & = & 2 \\ \text { 8._at: exp100_usd } & = & 80 \\ \text { tot_dry } & = & 3\end{array}$

\begin{tabular}{cc} 
& Margin \\
\hline 1._at & $-5.7913^{* * *}$ \\
& $(0.3489)$ \\
2._at & $-5.7444^{* * *}$ \\
& $(0.3423)$ \\
3._at & $-5.6975^{* * *}$ \\
& $(0.3517)$ \\
4._at & $-5.6506^{* * *}$ \\
& $(0.3758)$ \\
5._at & $26.9293^{* * *}$ \\
& $(7.6612)$ \\
6._at & $15.7557^{* * *}$ \\
& $(4.2471)$ \\
7._at & 4.5822 \\
& $(4.1786)$ \\
8._at & -6.5913 \\
& $(7.5474)$ \\
\hline$N$ & 29929 \\
\hline Standard errors in parentheses
\end{tabular}




$$
{ }^{*} p<0.10,{ }^{* *} p<0.05,{ }^{* * *} p<0.01
$$

The graph in Figure 10 shows the slopes of the number of time for an outpatient visit during dry month episodes when the economic condition variable (a coping factor being examined) is held constant at different combinations of values from very low to very high (100 and 8000 dollars per month).

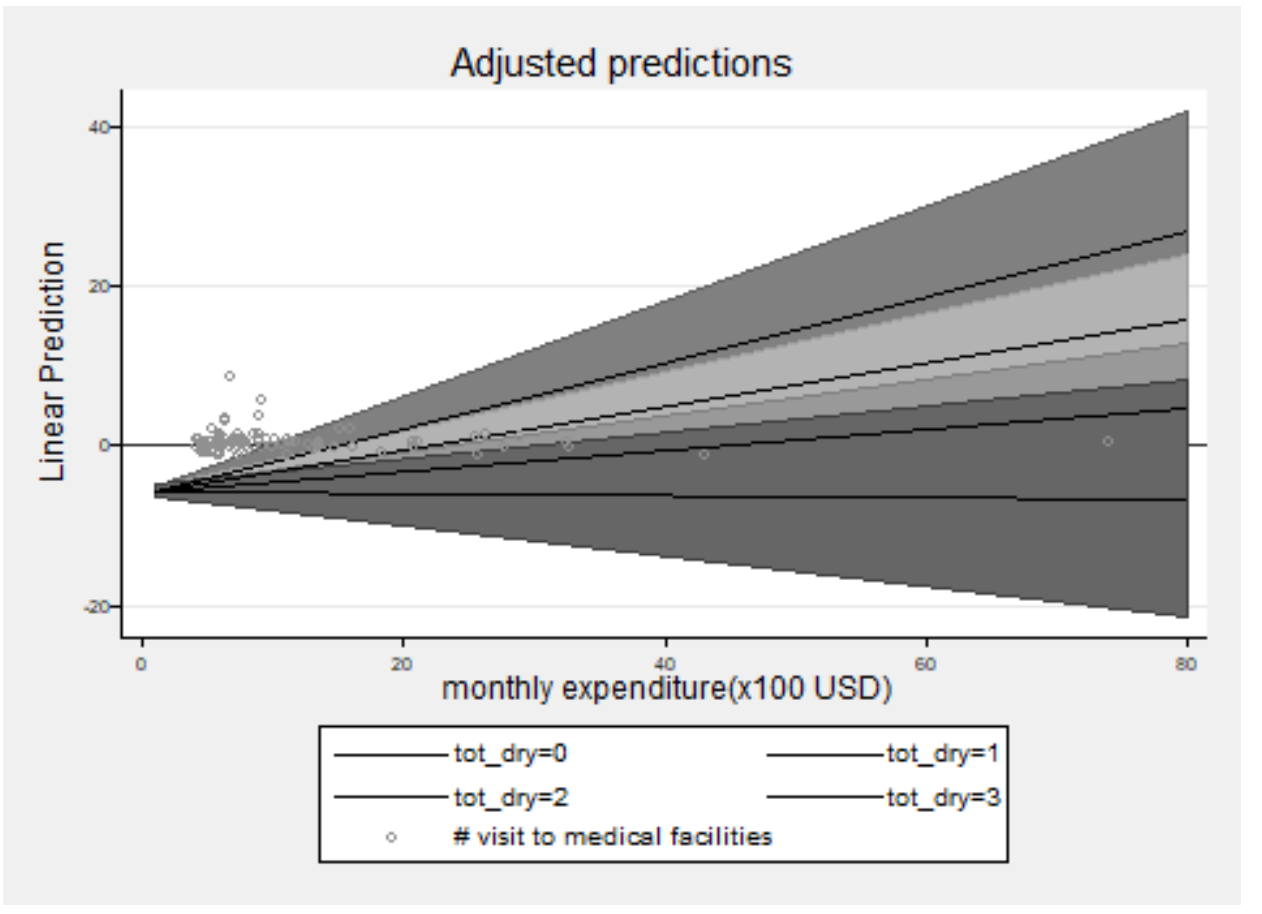

Figure 10 Effect of economic condition to reduce dry month episodes

Overall, there is little evidence that the coping strategies being examined are effective to alleviate the adverse impact of wet month episodes. There is a significant result though that suggest someone with more assets experiences less effect on outpatient cost (Table 18 , column cost and row interaction effect's Extreme dry X private health insurance). The negative 0.0004 coefficient regression suggests that each one hundred 100 dollar change in monthly expenditure, the effect of the wet month becomes 0.04 cents US dollar less on outpatient cost.

\subsubsection{Reviews on each coping strategies}

Next, having examined all coping strategies in one regression, this section further scrutinizes the effects of coping factors by specifying each factor in a regression model. 
The analysis estimates the interaction effects between each coping factor and extreme rainfall episode in the impacts on each outcome variable. Heterogeneous effects of the coping mechanism are shown when the estimations separately account each strategy on each outcome impact (see Annex 1-8). For example, monthly health expenditure of individuals with health insurance (either government scheme or private) is less affected during extreme weather than individuals without insurance. An individual enrolled in a government health insurance scheme has 66 cents US dollar less predicted impact than the one without insurance during wet months, and during dry month an individual who has private health insurance is impacted 69 cents US dollar less than the one without insurance. The welfare status also indicates the same mitigating effect during dry month episodes.

The effects of each coping strategies in reducing impacts of extreme weather in other outcomes are less robust and in many cases show non-reducing effects (indicated by positive interaction coefficients which mean someone with the coping factors would experience more severe impact, or more vulnerable than the other who does not have the coping factor). On the bedrest impact, for example, the only significant result is the effect of having private health insurance during dry months (see Annex 1) and other significant results show that coping factors (higher economic condition and higher farm income) escalate the impact levels than conditions without these coping factors.

\subsection{Conclusion}

The Sendai framework has renewed global attention on natural disaster's health impacts, especially for a coherent and effective climate adaptation and disaster reduction (DRR) strategy. Health focus has a solid provision of science and technology advancement to understand better hazard risks that help increase people's awareness and formulation of policy measure to reduce vulnerability being exposed by a disaster.

This paper examines the impacts of extreme rainfall conditions on several health outcomes and life satisfaction using panel methods. Specifically, it is focused on the effect of experiencing recurring excessive (wet month) and shortage rainfall (dry month) episodes in the last 3 months in a developing economy setting, which due to its socioeconomic characteristics and geographical location is prone to weather risk. The empirical specifications model the relationship that accounts for individual and time 
fixed-effects. The results indicate that there are statistically significant correlations between a combined wet extreme and dry extreme weather and mild symptoms such as a headache, runny nose, sore eyes, cough, cold, and nausea, as well as with digestive symptoms such as stomachache and diarrhea. The estimated contemporaneous effects on health also suggest that adult individuals exposed to dry extreme weather have a higher probability to suffer from respiratory symptom problems such as difficulty in breathing. Nevertheless, the regression results indicate no links between extreme rainfall and symptoms of infectious illness such as eye and skin infection.

Assessments on other health-related outcomes such as outpatient expenditure, bedrest, outpatient visits, and general health status indicate that it is only dry month episodes affect the health-related outcomes; such that excessive rainfall conditions do not have significant effects. This study finds that the frequencies of dry month episodes are significantly associated (at 95\% confidence level) with the changes in bedrest (number of days that one has to stay in bed due to illness). The estimates suggest that if there were 3 dry months in a row, someone who requires bedrest would have a 1.31 days rise in bed rest compared to days of bed rest in a normal weather condition. The rainfall effect on outpatient visits for outpatient care, although the coefficient is statistically significant, is very small ( 1 unit increase in dry month frequency predicts 0.066 times more of outpatient visits). The tiny effect of dry month and insignificance result of wet month episodes suggest that adverse weather conditions may not the influential factor for this health outcome. The other variables being controlled such as age, education level, having health insurance, seem to have a more significant influence on the number of outpatient visits than the weather variables.

The regression results of this article do not find a significant effect of extreme rainfall on health expenditure (for outpatient care) as well as on self-reported general health status. The insignificance estimates in individual's outpatient expenditure may be reflections of multitude determinants in economic decisions of an individual so that measuring at this level is difficult. Resource allocations in the family may be decided by and for other members in the household and in this case, may be allocated for other higher priority than health purposes. For example, during and after bad weather due to economic hardship because of crop losses, individual or household may have so that to choose between spending money on food or health care. 
One of the new features this study offers to the literature is the assessment of weather effects to life satisfaction, which is a proxy to an individual's well-being measured subjectively (SWB). Empirical analysis on SWB impact specifies several cross-sections (CS), pooled crossed section (pooled CS) and panel models - as life satisfaction data is available only after the last 2 waves of the survey (IFLS 2007 and 2014). The results suggest negative correlations in all models, but we think the results in panel model are more appropriate. The findings also indicate some positive relationships between rainfall anomalies and SWB. The positive results may be reflections of the agricultural-type economic driver in Indonesia which mostly find benefits from the conditions of abundant rain.

Finally, this study examines various coping mechanisms in the individual, and household, characteristics that may determine the vulnerability of the population against weatherrelated events. From the policy perspective, understanding these coping factors effects help formulate effective intervention measures to reduce the consequences of weather events. The findings suggest that there are heterogeneous effects with respect to, for example, the role of insurance. Importantly, the overall findings indicate mixed results between the effects of strategies during excessive rainfall conditions (wet months) and during rainfall shortage conditions (dry months). For example, having insurance does not have an effect that reduces the impact of wet months on outpatient expenditure, but it does have a mitigating effect during dry month episodes. This study sheds new lights on the potential consequences of recurring extreme weather conditions on the population's health and well-being being geographically and economically prone to weather risk. 


\section{Annexes Chapter FOUR}

Annex 1 Formal and informal insurance on outpatient expenditures' impact

\begin{tabular}{|c|c|c|c|c|c|}
\hline & \multicolumn{5}{|c|}{ Dependent Variable: outpatient cost (in USD) } \\
\hline & Govt HI & Prive HI & Welfare & Assets & Farm Inc \\
\hline Extreme wet months frequency $(0-3)$ & $\begin{array}{c}-0.1873 \\
(0.1204)\end{array}$ & $\begin{array}{c}-0.3226^{* * *} \\
(0.1044)\end{array}$ & $\begin{array}{c}-0.5383^{* * * *} \\
(0.1709)\end{array}$ & $\begin{array}{c}-0.2335^{* *} \\
(0.1012)\end{array}$ & $\begin{array}{c}-0.3565^{* * *} \\
(0.1074)\end{array}$ \\
\hline Extreme dry months frequency $(0-3)$ & $\begin{array}{c}-0.1921 \\
(0.1328)\end{array}$ & $\begin{array}{c}-0.1921 \\
(0.1253)\end{array}$ & $\begin{array}{l}-0.0846 \\
(0.1343)\end{array}$ & $\begin{array}{c}-0.1595 \\
(0.1271)\end{array}$ & $\begin{array}{l}-0.2121 \\
(0.1311)\end{array}$ \\
\hline Government health insurance & $\begin{array}{c}0.2321 \\
(0.3272)\end{array}$ & & & & \\
\hline Private health insurance & & $\begin{array}{c}0.4783 \\
(0.4768)\end{array}$ & & & \\
\hline HH monthly expenditure (HH welfare) & & & $\begin{array}{c}0.4885^{* * *} \\
(0.1653)\end{array}$ & & \\
\hline HH Total Assets per capita & & & & $\begin{array}{c}0.0018^{*} \\
(0.0010)\end{array}$ & \\
\hline HH Farm Income per capita & & & & & $\begin{array}{l}-0.0209 \\
(0.0372)\end{array}$ \\
\hline Extreme wet $\mathrm{X}$ govt. health insurance & $\begin{array}{l}-0.6595^{*} \\
(0.3783)\end{array}$ & & & & \\
\hline Extreme wet $\mathrm{X}$ private health insurance & & $\begin{array}{c}-0.0420 \\
(0.5540)\end{array}$ & & & \\
\hline Extreme wet X HH monthly expenditure & & & $\begin{array}{c}0.3071 \\
(0.2714)\end{array}$ & & \\
\hline Extreme wet X HH Total Assets per capita & & & & $\begin{array}{c}-0.0013^{*} \\
(0.0008)\end{array}$ & \\
\hline Extreme wet X HH Farm Income per capita & & & & & $\begin{array}{c}0.0301 \\
(0.0302)\end{array}$ \\
\hline Extreme dry X govt. health insurance & $\begin{array}{c}-0.3631 \\
(0.2217)\end{array}$ & & & & \\
\hline Extreme dry X private health insurance & & $\begin{array}{c}-0.6922^{* *} \\
(0.3156)\end{array}$ & & & \\
\hline Extreme dry X HH monthly expenditure & & & $\begin{array}{c}-0.1605^{* *} \\
(0.0660)\end{array}$ & & \\
\hline Extreme dry X HH Total Assets per capita & & & & $\begin{array}{c}-0.0008^{*} \\
(0.0005)\end{array}$ & \\
\hline Extreme dry X HH Farm Income per capita & & & & & $\begin{array}{c}0.0116 \\
(0.0276)\end{array}$ \\
\hline$N$ & 29,930 & 29,930 & 29,930 & 29,930 & 29,930 \\
\hline
\end{tabular}

Note: Clustered at sub-district standard errors in parentheses. ${ }^{*} p<0.10,{ }^{* *} p<0.05,{ }^{* * *} p<0.01$. Model specifications use Tobit regression as the dependent variable outpatient cost is skewed to the value of 0 , which suggest the majority of individuals does not seek for outpatient care, therefore, spending for this care is 0 (in USD). Several control variables that reflect individual and household characteristics are considered. These are hot month, age, household size, marriage status, gender, education, house ownership status, has electricity at home, and economic condition based on total monthly expenditure. Interaction effects are reported in rows with interacting signs, for example, extreme wet X govt. health insurance and Extreme dry X private health insurance. Control variables included in the regressions are the same as the ones included in Table 18 and 19. Extreme wet month frequency is the total number of the month when actual monthly precipitation exceeds the 50 years historic extreme upper threshold (p90) of cumulative rainfall in a month. Extreme dry month frequency is the total number of the month when monthly precipitation below the 50 years historic extreme lower threshold (p10) of monthly cumulative rainfall. 
Annex 2 Mitigating characteristics as coping strategies to reduce bedrest impact

\begin{tabular}{|c|c|c|c|c|c|}
\hline & \multicolumn{5}{|c|}{ Dependent Variable: number of days in bed because of sick } \\
\hline & Govt HI & Prive HI & Welfare & Assets & Farm Inc \\
\hline Extreme wet months frequency $(0-3)$ & $\begin{array}{c}0.4230 \\
(0.3055)\end{array}$ & $\begin{array}{c}0.0332 \\
(0.0367)\end{array}$ & $\begin{array}{c}0.1826 \\
(0.3571)\end{array}$ & $\begin{array}{c}0.2893 \\
(0.2830)\end{array}$ & $\begin{array}{c}0.5271^{* *} \\
(0.2680)\end{array}$ \\
\hline Extreme dry months frequency $(0-3)$ & $\begin{array}{l}0.5315^{* * *} \\
(0.1985)\end{array}$ & $\begin{array}{c}0.0237 \\
(0.0222)\end{array}$ & $\begin{array}{l}0.4902^{* * *} \\
(0.1895)\end{array}$ & $\begin{array}{l}0.3922^{* *} \\
(0.1843)\end{array}$ & $\begin{array}{c}0.4549^{* *} \\
(0.1828)\end{array}$ \\
\hline Government health insurance & $\begin{array}{l}1.0013^{* *} \\
(0.4898)\end{array}$ & $\begin{array}{c}0.0663 \\
(0.0461)\end{array}$ & $\begin{array}{l}0.6885^{* *} \\
(0.3458)\end{array}$ & $\begin{array}{l}0.6842^{* *} \\
(0.3456)\end{array}$ & $\begin{array}{l}0.6814^{* *} \\
(0.3454)\end{array}$ \\
\hline Private health insurance & $\begin{array}{c}0.2918 \\
(0.9276)\end{array}$ & $\begin{array}{c}0.0626 \\
(0.1113)\end{array}$ & $\begin{array}{c}0.2610 \\
(0.9265)\end{array}$ & $\begin{array}{c}0.3001 \\
(0.9278)\end{array}$ & $\begin{array}{c}0.2760 \\
(0.9269)\end{array}$ \\
\hline HH monthly expenditure (HH welfare) & $\begin{array}{c}0.1217 \\
(0.1015)\end{array}$ & $\begin{array}{c}0.0120 \\
(0.0116)\end{array}$ & $\begin{array}{c}0.2755 \\
(0.1901)\end{array}$ & $\begin{array}{c}0.1201 \\
(0.1009)\end{array}$ & $\begin{array}{c}0.1183 \\
(0.1007)\end{array}$ \\
\hline HH Total Assets per capita & $\begin{array}{c}-0.0018 \\
(0.0011)\end{array}$ & $\begin{array}{c}-0.0002^{* *} \\
(0.0001)\end{array}$ & $\begin{array}{l}-0.0020^{*} \\
(0.0012)\end{array}$ & $\begin{array}{l}-0.0030 \\
(0.0021)\end{array}$ & $\begin{array}{c}-0.0017 \\
(0.0011)\end{array}$ \\
\hline HH Farm Income per capita & $\begin{array}{c}0.0327 \\
(0.0540)\end{array}$ & $\begin{array}{c}0.0031 \\
(0.0071)\end{array}$ & $\begin{array}{c}0.0327 \\
(0.0540)\end{array}$ & $\begin{array}{c}0.0350 \\
(0.0542)\end{array}$ & $\begin{array}{c}0.1076 \\
(0.0761)\end{array}$ \\
\hline Extreme wet $\mathrm{X}$ govt. health insurance & $\begin{array}{l}-0.0240 \\
(0.5543)\end{array}$ & & & & \\
\hline Extreme wet $\mathrm{X}$ private health insurance & & $\begin{array}{c}-0.0168 \\
(0.1692)\end{array}$ & & & \\
\hline Extreme wet X HH monthly expenditure & & & $\begin{array}{c}0.3331 \\
(0.3286)\end{array}$ & & \\
\hline Extreme wet X HH Total Assets per capita & & & & $\begin{array}{c}0.0027 \\
(0.0023)\end{array}$ & \\
\hline Extreme wet X HH Farm Income per capita & & & & & $\begin{array}{c}-0.1130 \\
(0.0859)\end{array}$ \\
\hline Total dry X govt. health insurance & $\begin{array}{c}-0.5683 \\
(0.3816)\end{array}$ & & & & \\
\hline Total dry X private health insurance & & $\begin{array}{c}-0.1170^{*} \\
(0.0656)\end{array}$ & & & \\
\hline Total dry X HH monthly expenditure & & & $\begin{array}{c}-0.1190 \\
(0.0978)\end{array}$ & & \\
\hline Total dry X HH Total Assets per capita & & & & $\begin{array}{c}0.0002 \\
(0.0012)\end{array}$ & \\
\hline Total dry X HH Farm Income per capita & & & & & $\begin{array}{c}-0.0795 \\
(0.0636)\end{array}$ \\
\hline$N$ & 29,930 & 29,930 & 29,930 & 29,930 & 29,930 \\
\hline
\end{tabular}

Note: Clustered at sub-district standard errors in parentheses. ${ }^{*} \mathrm{p}<0.10,{ }^{* *} \mathrm{p}<0.05,{ }^{* * *} \mathrm{p}<0.01$. Model specifications account for individual and wave fixed-effect. Model specifications use Tobit regression as dependent variable bedrest is skewed to the value of 0 , which suggest the majority of individuals report that they do not have bedrest (0 days stay in bed because of sickness). Several control variables that reflect individual and household characteristics are considered. These are hot month, age, household size, marriage status, gender, education, house ownership status, has electricity at home, and economic condition based on total monthly expenditure. Interaction effects are reported in rows with interacting signs, for example, extreme wet X govt. health insurance and Extreme dry X private health insurance. Extreme wet month frequency is the total number of the month when actual monthly precipitation exceeds the 50 years historic extreme upper threshold (p90) of cumulative rainfall in a month. Extreme dry month frequency is the total number of the month when monthly precipitation below the 50 years historic extreme lower threshold (p10) of monthly cumulative rainfall. 
Annex 3 Mitigating characteristics as coping strategies to reduce the impact on the outpatient visit

\begin{tabular}{|c|c|c|c|c|c|}
\hline & \multicolumn{5}{|c|}{ Dependent Variable: outpatient visit } \\
\hline & Govt HI & Prive HI & Welfare & Assets & Farm Inc \\
\hline Extreme wet months frequency $(0-3)$ & $\begin{array}{c}-0.0178 \\
(0.0576)\end{array}$ & $\begin{array}{c}-0.0140 \\
(0.0494)\end{array}$ & $\begin{array}{l}-0.1254^{*} \\
(0.0730)\end{array}$ & $\begin{array}{c}-0.0027 \\
(0.0524)\end{array}$ & $\begin{array}{c}-0.0302 \\
(0.0509)\end{array}$ \\
\hline Extreme dry months frequency $(0-3)$ & $\begin{array}{c}0.0596 \\
(0.0391)\end{array}$ & $\begin{array}{c}0.0587 \\
(0.0360)\end{array}$ & $\begin{array}{l}0.1330^{* * *} \\
(0.0426)\end{array}$ & $\begin{array}{c}0.0704^{*} \\
(0.0365)\end{array}$ & $\begin{array}{c}0.0394 \\
(0.0367)\end{array}$ \\
\hline Government health insurance & $\begin{array}{l}0.5057^{* * *} \\
(0.0916)\end{array}$ & $\begin{array}{l}0.5145^{* * *} \\
(0.0702)\end{array}$ & $\begin{array}{l}0.5154^{* * *} \\
(0.0703)\end{array}$ & $\begin{array}{l}0.5141^{* * *} \\
(0.0702)\end{array}$ & $\begin{array}{l}0.5168^{* * *} \\
(0.0702)\end{array}$ \\
\hline Private health insurance & $\begin{array}{l}0.8257^{* * *} \\
(0.1486)\end{array}$ & $\begin{array}{l}0.7853^{* * *} \\
(0.1860)\end{array}$ & $\begin{array}{l}0.8165^{* * *} \\
(0.1482)\end{array}$ & $\begin{array}{l}0.8246^{* * *} \\
(0.1486)\end{array}$ & $\begin{array}{l}0.8270^{* * *} \\
(0.1485)\end{array}$ \\
\hline HH monthly expenditure ( $\mathrm{HH}$ welfare) & $\begin{array}{l}0.1181^{* * *} \\
(0.0383)\end{array}$ & $\begin{array}{l}0.1178^{* * *} \\
(0.0382)\end{array}$ & $\begin{array}{l}0.2384^{* * *} \\
(0.0738)\end{array}$ & $\begin{array}{l}0.1180^{* * *} \\
(0.0383)\end{array}$ & $\begin{array}{l}0.1181^{* * *} \\
(0.0383)\end{array}$ \\
\hline HH Total Assets per capita & $\begin{array}{c}0.0002^{*} \\
(0.0001)\end{array}$ & $\begin{array}{c}0.0002^{*} \\
(0.0001)\end{array}$ & $\begin{array}{c}0.0002 \\
(0.0001)\end{array}$ & $\begin{array}{l}0.0004^{* *} \\
(0.0002)\end{array}$ & $\begin{array}{l}0.0002^{* *} \\
(0.0001)\end{array}$ \\
\hline HH Farm Income per capita & $\begin{array}{c}-0.0035 \\
(0.0091)\end{array}$ & $\begin{array}{c}-0.0036 \\
(0.0091)\end{array}$ & $\begin{array}{l}-0.0030 \\
(0.0090)\end{array}$ & $\begin{array}{c}-0.0038 \\
(0.0091)\end{array}$ & $\begin{array}{c}-0.0215 \\
(0.0147)\end{array}$ \\
\hline Extreme wet $\mathrm{X}$ govt. health insurance & $\begin{array}{c}0.0198 \\
(0.1080)\end{array}$ & & & & \\
\hline Extreme wet $\mathrm{X}$ private health insurance & & $\begin{array}{c}0.0586 \\
(0.3002)\end{array}$ & & & \\
\hline Extreme wet X HH monthly expenditure & & & $\begin{array}{l}0.1512^{* *} \\
(0.0737)\end{array}$ & & \\
\hline Extreme wet X HH Total Assets per capita & & & & $\begin{array}{c}-0.0001 \\
(0.0002)\end{array}$ & \\
\hline Extreme wet X HH Farm Income per capita & & & & & $\begin{array}{c}0.0183 \\
(0.0137)\end{array}$ \\
\hline Total dry X govt. health insurance & $\begin{array}{c}0.0033 \\
(0.0727)\end{array}$ & & & & \\
\hline Total dry X private health insurance & & $\begin{array}{c}0.0688 \\
(0.2122)\end{array}$ & & & \\
\hline Total dry X HH monthly expenditure & & & $\begin{array}{c}-0.0959^{* * *} \\
(0.0338)\end{array}$ & & \\
\hline Total dry X HH Total Assets per capita & & & & $\begin{array}{c}-0.0002 \\
(0.0001)\end{array}$ & \\
\hline Total dry X HH Farm Income per capita & & & & & $\begin{array}{c}0.0259^{* *} \\
(0.0125)\end{array}$ \\
\hline$N$ & 29,930 & 29,930 & 29,930 & 29,930 & 29,930 \\
\hline
\end{tabular}

Note: Clustered at sub-district standard errors in parentheses. ${ }^{*} \mathrm{p}<0.10,{ }^{* *} \mathrm{p}<0.05,{ }^{* * *} \mathrm{p}<0.01$. Model specifications account for individual and wave fixed-effect. Model specifications use Tobit regression as the dependent variable doctor visit is skewed to the value of 0 , which suggest the majority of individuals does not seek for outpatient care $(0$ visits). The dependent variable is outpatient visit and several control variables that reflect individual and household characteristics are considered. These are hot month, age, household size, marriage status, gender, education, house ownership status, has electricity at home, and economic condition based on total monthly expenditure. Interaction effects are reported in rows with interacting signs, for example, extreme wet X govt. health insurance and Extreme dry $\mathrm{X}$ private health insurance. Extreme wet month frequency is the total number of the month when actual monthly precipitation exceeds the 50 years historic extreme upper threshold (p90) of cumulative rainfall in a month. Extreme dry month frequency is the total number of the month when monthly precipitation below the 50 years historic extreme lower threshold (p10) of monthly cumulative rainfall. 
Annex 4 Distribution of wet months and dry months
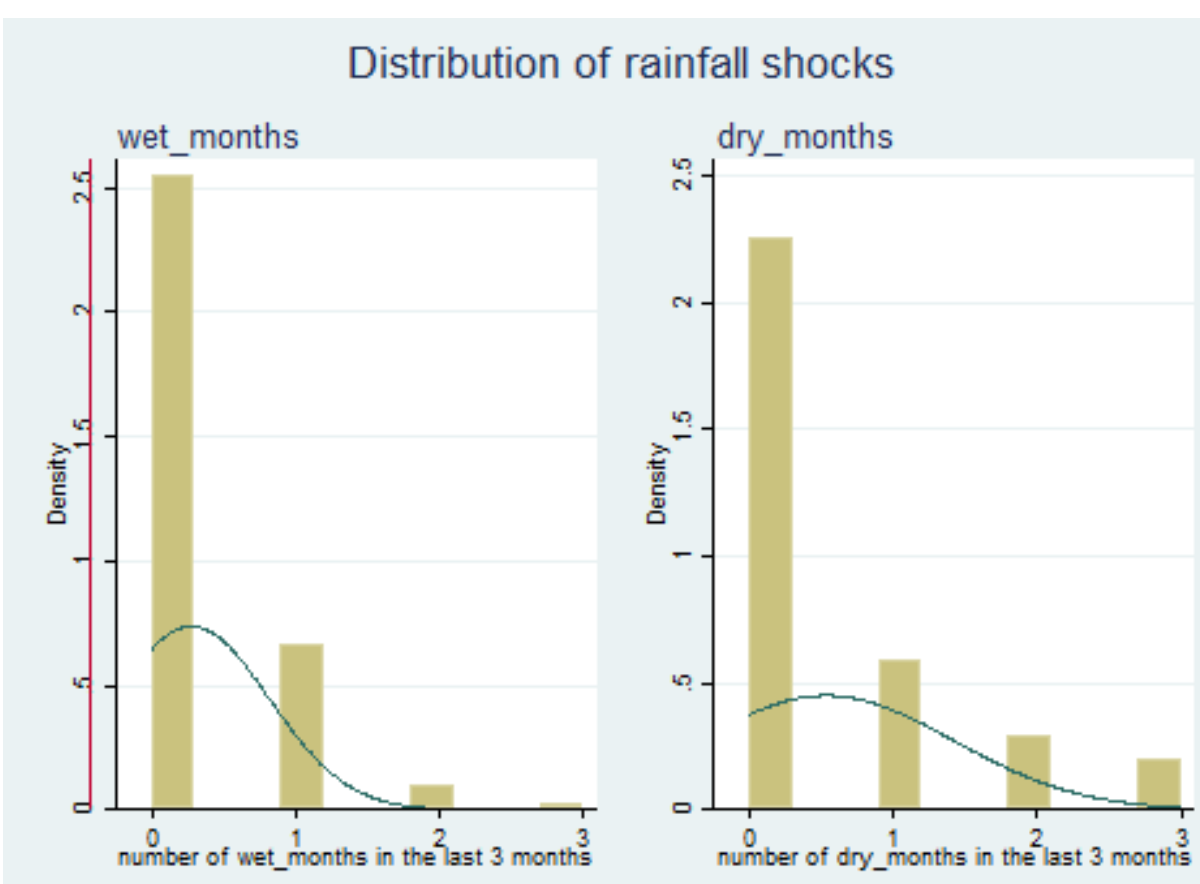

Annex 5 Distribution of outcome variables using tobit regression

Distribution outpatient cost, visit and bedrest
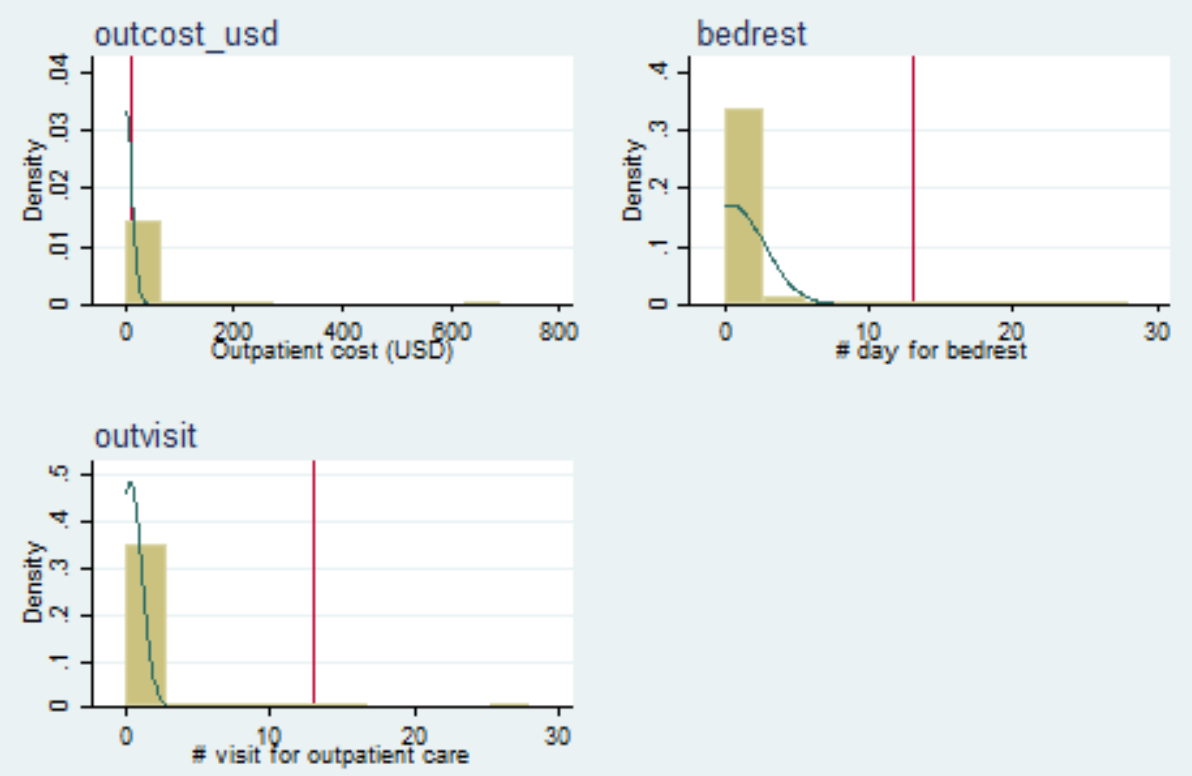
Annex 6 Distribution of outcome variables using ordered probit regression

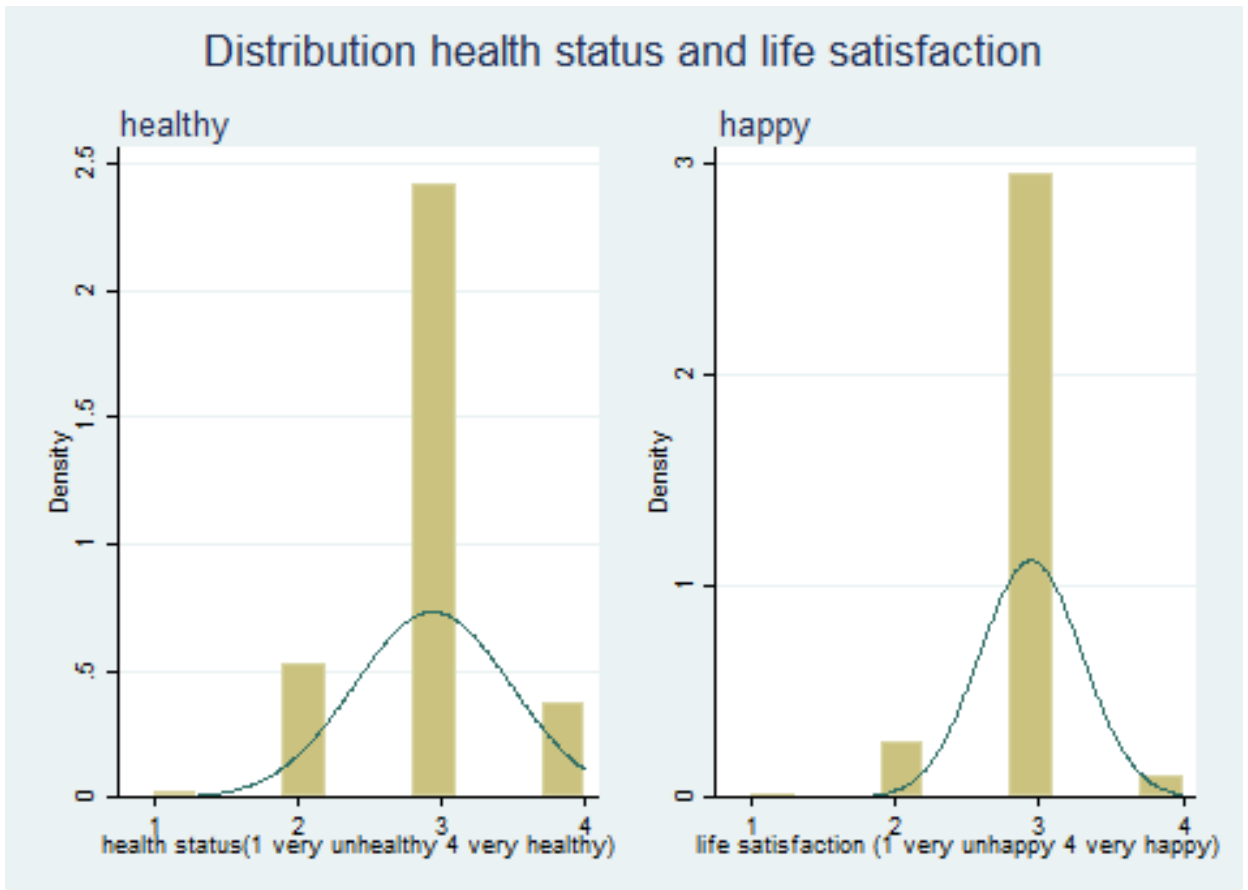




\section{Chapter FIVE}

\section{CONCLUSION}

The essays in this thesis have considered panel models in specifying the relationship between extreme weather events and economic outcomes, including health and life satisfaction. In an attempt to make progress in the research literature, the essays introduce new features in research designs, identification strategies, data and findings, which hopefully shed the new light about the impacts of anomalous weather conditions on social and economic activities. Further progress in scientific works helps people and policy makers to effectively react, anticipate and manage the weather risk.

One of the main features is the construction of exogenous weather shocks based on the extremes in the underlying weather variables being considered. The publicly available weather and climate indices used in the essays include Palmer Drought Severity Index (PDSI), Standardized Precipitation Evapotranspiration Index (SPEI) and CRU's monthly gridded rainfall data (CRU TS v. 4.01). The empirical analysis demonstrates the expediency of these indices for vigorously examining economic impacts of adverse weather events at detailed spatial resolutions. For example, to identify the consequences of recurring droughts at sub-district level, the second essay exploits variations in SPEI value -which also considers temperature variable- when it falls below negative 1 (indicating moderate dry spell condition) and under.

Overall findings suggest that the extremes in weather realizations have adverse effects on the observed outcomes such as agricultural yield, incomes, expenditures, and health. The timing of the shocks drives the consequences and each weather variables may have different impacts on one another. There is also a role of several coping mechanisms being examined, for example, insurance which can be useful for a policy reference. 


\section{REFERENCES}

Aitsi-Selmi, A., Egawa, S., Sasaki, H., Wannous, C., \& Murray, V. (2015). The Sendai Framework for Disaster Risk Reduction: Renewing the Global Commitment to People's Resilience, Health, and Well-being. International Journal of Disaster Risk Science, 6(2), 164-176. doi:10.1007/s13753-015-0050-9

Angelucci, M., De Giorgi, G., Rangel, M., \& Rasul, I. (2009). Village Economies and the Structure of Extended Family Networks The B.E. Journal of Economic Analysis \& Policy (Vol. 9).

Anselin, L. (1992). Special Issue Space and Applied EconometricsSpace and applied econometrics. Regional Science and Urban Economics, 22(3), 307-316.

Anselin, L. (2001). Spatial Effects in Econometric Practice in Environmental and Resource Economics. American Journal of Agricultural Economics, 83(3), 705-710.

Arcari, P., Tapper, N., \& Pfueller, S. (2007). Regional variability in relationships between climate and dengue/DHF in Indonesia. Singapore Journal of Tropical Geography, 28(3), 251-272. doi:doi:10.1111/j.1467-9493.2007.00300.x

Auffhammer, M., Hsiang, S. M., Schlenker, W., \& Sobel, A. (2013). Using Weather Data and Climate Model Output in Economic Analyses of Climate Change. National Bureau of Economic Research Working Paper Series, No. 19087. doi:10.3386/w19087

Auffhammer, M., \& Schlenker, W. (2014). Empirical studies on agricultural impacts and adaptation. Energy Economics, 46, 555-561. doi:https://doi.org/10.1016/i.eneco.2014.09.010

Baltagi, B. H., \& Hashem Pesaran, M. (2007). Heterogeneity and cross section dependence in panel data models: theory and applications introduction. Journal of Applied Econometrics, 22(2), 229-232.

Barrington-Leigh, C., \& Behzadnejad, F. (2017). The impact of daily weather conditions on life satisfaction: Evidence from cross-sectional and panel data. Journal of Economic Psychology, 59, 145-163. doi:https://doi.org/10.1016/j.joep.2017.01.003

Bastos, P., Busso, M., \& Miller, S. (2013). Adapting to climate change: Long-term effects of drought on local labor markets. IDB WORKING PAPER SERIES, No. IDB-WP-466.

Baudoin, M.-A., Vogel, C., Nortje, K., \& Naik, M. (2017). Living with drought in South Africa: lessons learnt from the recent El Niño drought period. International Journal of Disaster Risk Reduction, 23, 128-137.

Baum, C. F. (2004). HPRESCOTT: Stata module to implement Hodrick-Prescott filter for timeseries data (Version S447001): Boston College Department of Economics. Retrieved from https://ideas.repec.org/c/boc/bocode/s447001.html

Beguería, S., Vicente-Serrano, S. M., Reig, F., \& Latorre, B. (2014). Standardized precipitation evapotranspiration index (SPEI) revisited: parameter fitting, evapotranspiration models, tools, datasets and drought monitoring. International Journal of Climatology, 34(10), 3001-3023. doi:doi:10.1002/joc.3887

Berloffa, G., \& Modena, F. (2013). Income shocks, coping strategies, and consumption smoothing: An application to Indonesian data. Journal of Asian Economics, 24, 158171.

Bertram-Huemmer, V., \& Kraehnert, K. (2018). Does Index Insurance Help Households Recover from Disaster? Evidence from IBLI Mongolia. American Journal of Agricultural Economics, 100(1), 145-171. 
Binswanger-Mkhize, H. P. (2012). Is There Too Much Hype about Index-based Agricultural Insurance? Journal of Development Studies, 48(2), 187-200.

Born, B., \& Breitung, J. (2016). Testing for Serial Correlation in Fixed-Effects Panel Data Models. Econometric Reviews, 35(7), 1290-1316. doi:10.1080/07474938.2014.976524

Brereton, F., Clinch, J. P., \& Ferreira, S. (2008). Happiness, geography and the environment. Ecological Economics, 65(2), 386-396. doi:https://doi.org/10.1016/j.ecolecon.2007.07.008

Bruna, F., \& Yu, D. (2013). Geographically Weighted Panel Regression (GWPR). Paper presented at the 6th Seminar Jean Paelinck, Madrid.

Brunsdon, C., Fotheringham, A. S., \& Charlton, M. (1996). Geographically Weighted Regression: A Method for Exploring Spatial Nonstationarity. Geographical Analysis, 28(4), 281-298.

Brunsdon, C., Fotheringham, A. S., \& Charlton, M. (2002). Geographically Weighted Regression: The Analysis of Spatially Varying Relationships. The Statistician.

Bull, G. M., \& Morton, J. (1975). Relationships of Temperature with Death Rates from all Causes and from certain Respiratory and Arteriosclerotic Diseases in Different Age Groups. Age and Ageing, 4(4), 232-246. doi:10.1093/ageing/4.4.232

Bull, G. M., \& Morton, J. (1978). Environment, Temperature and Death rates. Age Ageing, $7(4), 210-224$.

Burgess, R., Deschenes, O., Donaldson, D., \& Greenstone, M. (2017). Weather, climate change and death in India. University of Chicago.

Cai, R., Yu, D., \& Oppenheimer, M. (2014). Estimating the Spatially Varying Responses of Corn Yields to Weather Variations Using Geographically Weighted Panel Regression. Journal of Agricultural and Resource Economics, 39(2), 230-252.

Carter, M., Janvry, A. d., Sadoulet, E., \& Sarris, A. (2017). Index Insurance for Developing Country Agriculture: A Reassessment. Annual Review of Resource Economics, 9(1), 421-438.

Caruso, R., Petrarca, I., \& Ricciuti, R. (2016). Climate change, rice crops, and violence:Evidence from Indonesia. Journal of Peace Research, 53(1), 66-83.

Cavallo, E., Galiani, S., Noy, I., \& Pantano, J. (2013). Catastrophic Natural Disasters and Economic Growth. Review of Economics and Statistics, 95(5), 1549--1561.

Ceglar, A., Toreti, A., Lecerf, R., Van der Velde, M., \& Dentener, F. (2016). Impact of meteorological drivers on regional inter-annual crop yield variability in France. Agricultural and Forest Meteorology, 216, 58-67.

Choudhury, A., Jones, J., Okine, A., \& Choudhury, R. (2016). Drought-Triggered Index Insurance Using Cluster Analysis of Rainfall Affected by Climate Change. Journal of Insurance Issues, 39(2), 169-186.

Clarke, D. J., Mahul, O., Rao, K. N., \& Verma, N. (2012). Weather Based Crop Insurance in India: The World Bank.

Collier, B., Skees, J., \& Barnett, B. J. (2009). Weather Index Insurance and Climate Change: Opportunity and Challenges in Lower Income Countries. The Geneva Papers, 34, 401424.

Conradt, S., Finger, R., \& Spörri, M. (2015). Flexible weather index-based insurance design. Climate Risk Management, 10, 106-117. 
D'Arrigo, R., \& Wilson, R. (2008). El Niño and Indian Ocean influences on Indonesian drought: implications for forecasting rainfall and crop productivity. International Journal of Climatology, 28(5), 611-616.

Dai, A., Trenberth, K. E., \& Qian, T. (2004). A Global Dataset of Palmer Drought Severity Index for 1870-2002: Relationship with Soil Moisture and Effects of Surface Warming. Journal of Hydrometeorology, 5(6), 1117-1130.

De Alwis, D., \& Noy, I. (2017). The cost of being under the weather: Droughts, floods, and health care costs in Sri Lanka.

Dell, M., Jones, B. F., \& Olken, B. A. (2014). What do we learn from the weather? The new climate-economy literature. Journal of Economic Literature, 52(3), 740-798.

Dercon, S., Hill, R. V., Clarke, D., Outes-Leon, I., \& Seyoum Taffesse, A. (2014). Offering rainfall insurance to informal insurance groups: Evidence from a field experiment in Ethiopia. Journal of Development Economics, 106, 132-143.

Deschenes, O. (2014). Temperature, human health, and adaptation: A review of the empirical literature. Energy Economics, 46, 606-619. doi:https://doi.org/10.1016/i.eneco.2013.10.013

Deschenes, O., \& Greenstone, M. (2007). The Economic Impacts of Climate Change: Evidence from Agricultural Output and Random Fluctuations in Weather. American Economic Review, 97(1), 354-385.

Devereux, S. (2007). The impact of droughts and floods on food security and policy options to alleviate negative effects. Agricultural Economics, 37(S1), 47--58.

Driscoll, J. C., \& Kraay, A. C. (1998). Consistent covariance matrix estimation with spatially dependent panel data. Review of Economics and Statistics, 80(4), 549-560.

Eeckhoudt, L., \& Gollier, C. (1995). Risk: evaluation, management and sharing. New York: Harvester Wheatsheaf.

Eisenberg, M. C., Kujbida, G., Tuite, A. R., Fisman, D. N., \& Tien, J. H. (2013). Examining rainfall and cholera dynamics in Haiti using statistical and dynamic modeling approaches. Epidemics, 5(4), 197-207. doi:https://doi.org/10.1016/j.epidem.2013.09.004

Estiningtyas, W., Boer, R., Las, I., Buono, A., \& Rakhman, A. (2011). Deliniasi Risiko Iklim dan Evaluasi Model Hubungan Curah Hujan dan Produksi Padi dalam Mendukung Pengembangan Asuransi Berbasis Indeks Iklim (Climate Index Insurance) Pada Sistem Usahatani Berbasis Padi. Jurnal Ilmu Pertanian Indonesia, 16(3), 198-208.

FAO. (2015). The Impact of Natural Hazards and Disasters on Agriculture and Nutrition and Food Security. Retrieved from Roma, Italy: http://www.fao.org/3/a-i4434e.pdf

Felbermayr, G., \& Gröschl, J. (2014). Naturally Negative: The Growth Effects of Natural Disasters. Journal of Development Economics, 111, 92-106.

Fotheringham, A. S., Brunsdon, C., \& Charlton, M. (2002). Geographically Weighted Regression: The Analysis of Spatially Varying Relationships: Wiley.

Fotheringham, A. S., Charlton, M. E., \& Brunsdon, C. (1998). Geographically Weighted Regression: A Natural Evolution of the Expansion Method for Spatial Data Analysis. Environment and Planning A: Economy and Space, 30(11), 1905-1927.

Fotheringham, A. S., Yang, W., \& Kang, W. (2017). Multiscale Geographically Weighted Regression (MGWR). Annals of the American Association of Geographers, 107(6), 1247-1265.

Fraley, C., \& Raftery, A. E. (2012). MCLUST: Software for Model-Based Cluster Analysis (Vol. 16, pp. 297): Springer Science \& Business Media B.V. 
Freire-González, J., Decker, C., \& Hall, J. W. (2017). The Economic Impacts of Droughts: A Framework for Analysis. Ecological Economics, 132, 196-204.

doi:https://doi.org/10.1016/j.ecolecon.2016.11.005

Galloway, P. R. (1985). Annual Variations in Deaths by Age, Deaths by Cause, Prices, and Weather in London 1670 to 1830. Population Studies, 39(3), 487-505. doi:10.1080/0032472031000141646

Gignoux, J., \& Menéndez, M. (2016). Benefit in the wake of disaster: Long-run effects of earthquakes on welfare in rural Indonesia. Journal of Development Economics, 118(Supplement C), 26-44.

Groppo, V., \& Kraehnert, K. (2016). Extreme Weather Events and Child Height: Evidence from Mongolia. World Development, 86, 59-78.

Haines, A., McMichael, A. J., Smith, K. R., Roberts, I., Woodcock, J., Markandya, A., . . . Wilkinson, P. (2009). Public health benefits of strategies to reduce greenhouse-gas emissions: overview and implications for policy makers. The Lancet, 374(9707), 2104-2114. doi:https://doi.org/10.1016/S0140-6736(09)61759-1

Hajat, S., Armstrong, B. G., Gouveia, N., \& Wilkinson, P. (2005). Mortality Displacement of Heat-Related Deaths: A Comparison of Delhi, São Paulo, and London. Epidemiology, 16(5), 613-620. doi:10.1097/01.ede.0000164559.41092.2a

Hanandita, W., \& Tampubolon, G. (2014). Does poverty reduce mental health? An instrumental variable analysis. Social Science \& Medicine, 113, 59-67. doi:https://doi.org/10.1016/i.socscimed.2014.05.005

Harris, I., Jones, P. D., Osborn, T. J., \& Lister, D. H. (2014). Updated high-resolution grids of monthly climatic observations - the CRU TS3.10 Dataset. International Journal of Climatology, 34(3), 623-642. doi:doi:10.1002/joc.3711

Heimfarth, L. E., \& Musshoff, O. (2011). Weather index-based insurances for farmers in the north China Plain. Agricultural Finance Review, 71(2), 218-239.

Hidayat, B., Thabrany, H., Dong, H., \& Sauerborn, R. (2004). The effects of mandatory health insurance on equity in access to outpatient care in Indonesia. Health Policy and Planning, 19(5), 322-335. doi:10.1093/heapol/czh037

Hodler, R., \& Raschky, P. A. (2014). Economic shocks and civil conflict at the regional level. Economics Letters, 124(3), 530-533.

Hoechle, D. (2007). Robust standard errors for panel regressions with cross-sectional dependence. Stata Journal, 7(3), 281.

Homdee, T., Pongput, K., \& Kanae, S. (2016). A comparative performance analysis of three standardized climatic drought indices in the Chi River basin, Thailand. Agriculture and Natural Resources, 50(3), 211-219. doi:https://doi.org/10.1016/i.anres.2016.02.002

Hsiang, S. M., Burke, M., \& Miguel, E. (2013). Quantifying the Influence of Climate on Human Conflict. Science, 341(6151). doi:10.1126/science.1235367

Hsiao, C. (2014). Analysis of Panel Data: Cambridge University Press.

IFC. (2012). Weather Index Insurance for Maize Production in Eastern Indonesia, a Feasibility Study. Retrieved from Jakarta:

http://www.ifc.org/wps/wcm/connect/ce97fd80472308938250ab2b131bed2a/Wea ther\%2BIndex\%2BInsurance\%2BReport.pdf?MOD=AJPERES.

lizumi, T., Luo, J.-J., Challinor, A. J., Sakurai, G., Yokozawa, M., Sakuma, H., . . Yamagata, T. (2014). Impacts of El Niño Southern Oscillation on the global yields of major crops. Nat Commun, 5. 
Insyafiah and Wardhani, I. (2014). Kajian Persiapan Implementasi Asuransi Pertanian Secara Nasional. Jakarta: Ministry of Finance of Republic of Indonesia Retrieved from https://www.kemenkeu.go.id/sites/default/files/kajian\%20persiapan\%20implement asi\%20asuransi\%20pertanian.pdf.

IPCC. (2012). Managing the Risks of Extreme Events and Disasters to Advance Climate Change Adaptation. Retrieved from https://www.ipcc.ch/pdf/specialreports/srex/SREX Full Report.pdf

Janvry, A. d., Ritchie, E. R., \& Sadoulet, E. (2016). Weather Index Insurance and Shock Coping, Evidence from Mexico's CADENA Program. World Bank Policy Research Working paper, No. 7715.

Karim, A. (2018). The Household Response to Persistent Natural Disasters: Evidence from Bangladesh. World Development, 103, 40-59. doi:https://doi.org/10.1016/j.worlddev.2017.10.026

Kathleen Beegle, Weerdt, Joachim D., \& Stefan Dercon. (2008). Adult Mortality and Consumption Growth in the Age of HIV/AIDS. Economic Development and Cultural Change, 56(2), 299-326.

Kawanishi, M., \& Mimura, N. (2015). Assessment of insurance for paddy production: a case study in Indonesia. Climate and Development, 7(3), 257-266.

Kelejian, H. H., \& Prucha, I. R. (2010). Specification and estimation of spatial autoregressive models with autoregressive and heteroskedastic disturbances. Journal of Econometrics, 157(1), 53-67.

Kellner, U., \& Musshoff, O. (2011). Precipitation or water capacity indices? An analysis of the benefits of alternative underlyings for index insurance. Agricultural Systems, 104(8), 645-653.

Kezdi, G. (2003). Robust standard error estimation in fixed-effects panel models.

Khalil, A. F., Kwon, H.-H., Lall, U., Miranda, M. J., \& Skees, J. (2007). El Niño-Southern Oscillation-based index insurance for floods: Statistical risk analyses and application to Peru. Water Resources Research, 43(10).

Kim, Y., Knowles, S., Manley, J., \& Radoias, V. (2017). Long-run health consequences of air pollution: Evidence from Indonesia's forest fires of 1997. Economics \& Human Biology, 26, 186-198. doi:https://doi.org/10.1016/j.ehb.2017.03.006

Korkeala, O., Newhouse, D. L., \& Duarte, M. (2009). Distributional Impact Analysis of Past Climate Variability in Rural Indonesia. World Bank Policy Research Working Paper Series No.5070.

Kwon, H. j., \& Kim, W. r. (2015). The Evolution of Cash Transfers in Indonesia: Policy Transfer and National Adaptation. Asia \& the Pacific Policy Studies, 2(2), 425-440.

Lanjouw, J. O., \& Lanjouw, P. (2001). The rural non-farm sector: issues and evidence from developing countries. Agricultural Economics, 26(1), 1-23. doi:doi:10.1111/j.15740862.2001.tb00051.x

Lassa, J. A. (2012). Emerging 'Agricultural Involution' in Indonesia: Impact of Natural Hazards and Climate Extremes on Agricultural Crops and Food System. Economic and Welfare Impacts of Disasters in East Asia and Policy Responses, Jakarta: ERIA, 601-640.

Leblois, A., \& Quirion, P. (2013). Agricultural insurances based on meteorological indices: Realizations, Methods and research challenges. Meteorological Applications, 20(1), 1-9.

LeSage, J. P., \& Dominguez, M. (2012). The importance of modeling spatial spillovers in public choice analysis. Public Choice, 150(3), 525-545. 
Letta, M., Montalbano, P., \& Tol, R. S. J. (2018). Temperature shocks, short-term growth and poverty thresholds: Evidence from rural Tanzania. World Development, 112, 13-32. doi:https://doi.org/10.1016/i.worlddev.2018.07.013

Lo lacono, G., Armstrong, B., Fleming, L. E., Elson, R., Kovats, S., Vardoulakis, S., \& Nichols, G. L. (2017). Challenges in developing methods for quantifying the effects of weather and climate on water-associated diseases: A systematic review. PLOS Neglected Tropical Diseases, 11(6), e0005659. doi:10.1371/journal.pntd.0005659

Lohmann, S., \& Lechtenfeld, T. (2015). The Effect of Drought on Health Outcomes and Health Expenditures in Rural Vietnam. World Development, 72, 432-448.

Maccini, S., \& Yang, D. (2009). Under the weather: Health, schooling, and economic consequences of early-life rainfall. The American Economic Review, 99(3), 10061026.

Maddison, D., \& Rehdanz, K. (2011). The impact of climate on life satisfaction. Ecological Economics, 70(12), 2437 - 2445. doi:https://doi.org/10.1016/j.ecolecon.2011.07.027

Mahul, O. (2001). Optimal insurance against climatic experience. American Journal of Agricultural Economics, 83(3), 593-604.

Mahul, O., \& Stutley, C. J. (2010). Government Support to Agricultural Insurance: Challenges and Options for Developing Countries. Washington, DC.: World Bank Publications.

Mani, S. (2012). Is there Complete, Partial, or No Recovery from Childhood Malnutrition? Empirical Evidence from Indonesia*. Oxford Bulletin of Economics and Statistics, 74(5), 691-715.

Maystadt, J.-F., \& Ecker, O. (2014). Extreme Weather and Civil War: Does Drought Fuel Conflict in Somalia through Livestock Price Shocks? American Journal of Agricultural Economics, 96(4), 1157-1182.

Mboi, N., Murty Surbakti, I., Trihandini, I., Elyazar, I., Houston Smith, K., Bahjuri Ali, P., . . Hay, S. I. (2018). On the road to universal health care in Indonesia, 1990-2016: a systematic analysis for the Global Burden of Disease Study 2016. The Lancet, 392(10147), 581-591. doi:https://doi.org/10.1016/S0140-6736(18)30595-6

Mclver, L., Kim, R., Woodward, A., Hales, S., Spickett, J., Katscherian, D., . . Ebi, K. L. (2016). Health Impacts of Climate Change in Pacific Island Countries: A Regional Assessment of Vulnerabilities and Adaptation Priorities. Environmental Health Perspectives, 124(11), 1707-1714. doi:doi:10.1289/ehp.1509756

Miguel, E., Satyanath, S., \& Sergenti, E. (2004). Economic Shocks and Civil Conflict: An Instrumental Variables Approach. Journal of Political Economy, 112(4), 725-753.

Miranda, M., \& Vedenov, D. V. (2001). Innovations in Agricultural and Natural Disaster Insurance. American Journal of Agricultural Economics, 83(3), 650-655.

Miranda, M. J., \& Farrin, K. (2012). Index Insurance for Developing Countries. Applied Economic Perspectives and Policy, 34(3), 391-427.

Naylor, R. L., Battisti, D. S., Vimont, D. J., Falcon, W. P., \& Burke, M. B. (2007). Assessing risks of climate variability and climate change for Indonesian rice agriculture. Proceedings of the National Academy of Sciences, 104(19), 7752-7757.

Naylor, R. L., Falcon, W. P., Rochberg, D., \& Wada, N. (2001). Using El Niño/Southern Oscillation Climate Data to predict Rice production in Indonesia. Climatic Change, 50, 255-265.

Nieto, J. D., Cook, S. E., Läderach, P., Fisher, M. J., \& Jones, P. G. (2010). Rainfall index insurance to help smallholder farmers manage drought risk. Climate and Development, 2(3), 233-247. 
Noy, I., Kusuma, A., \& Nguyen, C. (2017). Insuring disasters: A survey of the economics of insurance programs for earthquakes and droughts. Retrieved from https://ideas.repec.org/p/vuw/vuwecf/6408.html

Noy, I., \& Vu, T. B. (2010). The economics of natural disasters in a developing country: The case of Vietnam. Journal of Asian Economics, 21(4), 345-354.

Otrachshenko, V., Popova, O., \& Solomin, P. (2018). Misfortunes never come singly: Consecutive weather shocks and mortality in Russia. Economics \& Human Biology, 31, 249-258. doi:https://doi.org/10.1016/j.ehb.2018.08.008

Owen, S., \& Noy, I. (2017). The unfortunate regressivity of public natural hazard insurance: A quantitative analysis of a New Zealand case. (6399).

Palmer, W. C. (1965). Meteorological drought. Research Paper 45, U.S. Dept. of CommerCE, $55 \mathrm{pp}$.

Patz, J. A., Engelberg, D., \& Last, J. (2000). The Effects of Changing Weather on Public Health. Annual Review of Public Health, 21(1), 271-307. doi:10.1146/annurev.publhealth.21.1.271

Paulson, N. D., Hart, C. E., \& Hayes, D. J. (2010). A Spatial Bayesian approach to weather derivatives. Agricultural Finance Review, 70(1), 79-96.

Pesaran, M. H. (2015). Testing Weak Cross-Sectional Dependence in Large Panels. Econometric Reviews, 34(6-10), 1089-1117. doi:10.1080/07474938.2014.956623

Post, J. D. (1984). Climatic Variability and the European Mortality Wave of the Early 1740 s. The Journal of Interdisciplinary History, 15(1), 1-30. doi:10.2307/203592

Ranson, M. (2014). Crime, weather, and climate change. Journal of Environmental Economics and Management, 67(3), 274-302.

Rao, M. P., Davi, N. K., D'Arrigo, R. D., Skees, J., Nachin, B., Leland, C., . . Byambasuren, O. (2015). Dzuds, droughts, and livestock mortality in Mongolia. Environmental Research Letters, 10(7), 074012.

Reardon, T., Stamoulis, K., Balisacan, A., Cruz, M., Berdegué, J., \& Banks, B. (1998). Rural non-farm income in developing countries. The state of food and agriculture, 1998, 283-356.

Rehdanz, K., \& Maddison, D. (2005). Climate and happiness. Ecological Economics, 52(1), 111-125. doi:https://doi.org/10.1016/i.ecolecon.2004.06.015

Rejeki, D. S. S., Nurhayati, N., Aji, B., Murhandarwati, E. E. H., \& Kusnanto, H. (2018). A Time Series Analysis: Weather Factors, Human Migration and Malaria Cases in Endemic Area of Purworejo, Indonesia, 2005-2014. Iranian journal of public health, 47(4), 499509.

Rosenzweig, C. E., Ruane, A. C., Glotter, M., Boote, K. J., Arneth, A., Folberth, C., . . . Khabarov, N. (2014). Assessing Agricultural Risks of Climate Change in the 21st Century in a Global Gridded Crop Model Intercomparison. Paper presented at the National Academy of Sciences of the United States of America.

Sarafidis, V., \& Wansbeek, T. (2012). Cross-sectional dependence in panel data analysis. Econometric Reviews, 31(5), 483-531.

Shah, M., \& Steinberg, B. M. (2017). Drought of Opportunities: Contemporaneous and LongTerm Impacts of Rainfall Shocks on Human Capital. Journal of Political Economy, 125(2), 527--561.

Shi, H., \& Jiang, Z. (2016). The efficiency of composite weather index insurance in hedging rice yield risk: evidence from China. Agricultural Economics, 47(3), 319-328. 
Skees, J., Hazell, P. B. R., \& Miranda, M. (1999). New Approaches to Crop Yield Insurance in Developing Countries. Discussion paper, International Food Policy Research Institute, Environment and Production Technology Division: Washington, DC., 55.

Skees, J. R., Black, J. R., \& Barnett, B. J. (1997). Designing and Rating an Area Yield Crop Insurance Contract. American Journal of Agricultural Economics, 79(2), 430-438.

Skees, J. R., Varangis, D., Larson, D., \& Siegel, P. (2004). Can Financial Markets Be Tapped to Help Poor People Cope With Weather Risks? Insurance Against Poverty. WIDER Studies in Development Economics.

Skees, J. R., Varangis, P., Gober, S., Lester, R., \& Kalavakonda, V. (2001). Developing RainfallBased Index Insurance in Morocco: World Bank, Middle East and North Africa Region, Private Sector Development and Finance Group; Development Research Group, Rural Development; Financial Sector Department.

Skoufias, E. (2003). Economic Crises and Natural Disasters: Coping Strategies and Policy Implications. World Development, 31(7), 1087-1102.

Skoufias, E., Katayama, R. S., \& Essama-Nssah, B. (2012). Too little too late: welfare impacts of rainfall shocks in rural Indonesia. Bulletin of Indonesian Economic Studies, 48(3), 351-368.

Skoufias, E., \& Vinha, K. (2012). Climate variability and child height in rural Mexico. Economics \& Human Biology, 10(1), 54-73.

Smith, V., \& Watts, M. (2009). Index Based Agricultural Insurance in Developing Countries: Feasibility, Scalability and Sustainability. Report to the Bill and Melinda Gates Foundation.

Solh, M., \& van Ginkel, M. (2014). Drought preparedness and drought mitigation in the developing world's drylands. Weather and Climate Extremes, 3, 62-66.

Sparrow, R., Suryahadi, A., \& Widyanti, W. (2013). Social health insurance for the poor: Targeting and impact of Indonesia's Askeskin programme. Social Science \& Medicine, 96, 264-271. doi:https://doi.org/10.1016/i.socscimed.2012.09.043

Spicka, J., \& Hnilica, J. (2013). A Methodical Approach to Design and Valuation of Weather Derivatives in Agriculture. Advances in Meteorology, 2013, 8.

Spinoni, J., Naumann, G., Vogt, J. V., \& Barbosa, P. (2015). The biggest drought events in Europe from 1950 to 2012. Journal of Hydrology: Regional Studies, 3, 509-524. doi:https://doi.org/10.1016/j.ejrh.2015.01.001

Stagge, J. H., Tallaksen, L. M., Gudmundsson, L., Van Loon, A. F., \& Stahl, K. (2015). Candidate Distributions for Climatological Drought Indices (SPI and SPEI). International Journal of Climatology, 35(13), 4027-4040. doi:doi:10.1002/joc.4267

Strauss, J., Beegle, K., Dwiyanto, A., Herawati, Y., Pattinasarany, D., Satriawan, E., \& Sikoki, B. (2002). Indonesian living standards three years after the crisis: evidence from the Indonesia Family Life Survey.

Strauss, J., Witoelar, F., \& Sikoki, B. (2016). The fifth wave of the Indonesia family life survey: Overview and field report (Vol. WR-1143/1-NIA/NICHD).

Strobl, E. (2012). The Economic Growth Impact of Natural Disasters in Developing Countries: Evidence From Hurricane Strikes in the Central American and Caribbean Regions. Journal of Development Economics, 97(1), 130-141.

Surmaini, E., Hadi, T. W., Subagyono, K., \& Puspito, N. T. (2015). Early detection of drought impact on rice paddies in Indonesia by means of Niño 3.4 index. Theoretical and Applied Climatology, 121(3), 669--684. 
Tadesse, M. A., Shiferaw, B. A., \& Erenstein, O. (2015). Weather Index Insurance For Managing Drought Risk In Smallholder Agriculture: Lessons and Policy Implications For Sub-Saharan Africa. AGRICULTURAL AND FOOD ECONOMICS, 3(1), 26.

Thomas, D., Witoelar, F., Frankenberg, E., Sikoki, B., Strauss, J., Sumantri, C., \& Suriastini, W. (2012). Cutting the costs of attrition: Results from the Indonesia Family Life Survey. Journal of Development Economics, 98(1), 108-123.

UNFCCC. (2008). Mechanisms to Manage Financial Risks From Direct Impacts Of Climate Change in Developing Countries (FCCC/TP/2008/9). Retrieved from http://unfccc.int/resource/docs/2008/tp/09.pdf

UNISDR. (2015a). Global Assessment Report on Disaster Risk Reduction, Making Development Sustainable: The Future of Disaster Risk Management. Geneva: The United Nations Office for Disaster Risk Reduction (UNISDR).

UNISDR. (2015b). Sendai framework for disaster risk reduction 2015-2030. United Nations International Strategy for Disaster Reduction Retrieved from https://www.preventionweb.net/files/43291 sendaiframeworkfordrren.pdf.

Vicente-Serrano, S. M., Beguera, S., \& Lpez-Moreno, J. I. (2010). A Multi-scalar Drought Index Sensitive to Global Warming: The Standardized Precipitation Evapotranspiration Index. Journal of Climate, 23(7), 1696--1718.

Vicente-Serrano, S. M., Beguera, S., Lpez-Moreno, J. I., Angulo, M., \& El Kenawi, A. (2010). A New Global $0.5^{\circ}$ Gridded Dataset (1901-2006) of a Multiscalar Drought Index: Comparison with Current Drought Index Datasets Based on the Palmer Drought Severity Index. Journal of Hydrometeorology, 11(4), 1033--1043.

Wang, K.-Y., Li, Q.-f., Yang, Y., Zeng, M., Li, P.-c., \& Zhang, J.-x. (2015). Analysis of spatiotemporal evolution of droughts in Luanhe River Basin using different drought indices. Water Science and Engineering, 8(4), 282-290. doi:https://doi.org/10.1016/j.wse.2015.11.004

WHO. (2014). Health and disaster risk: a contribution by the United Nations to the consultation leading to the Third UN World Conference on Disaster Risk Reduction. World Health Organization, Accessed Apr 2015.

Witoelar, F. (2013). Risk Sharing within the Extended Family: Evidence from the Indonesia Family Life Survey. Economic Development and Cultural Change, 62(1), 65-94.

Woodard, J. D., Shee, A., \& Mude, A. (2016). A Spatial Econometric Approach to Designing and Rating Scalable Index Insurance in the Presence of Missing Data. The Geneva Papers on Risk and Insurance - Issues and Practice, 41(2), 259-279.

Wu, M., Chen, Y., Wang, H., \& Sun, G. (2015). Characteristics of meteorological disasters and their impacts on the agricultural ecosystems in the northwest of China: a case study in Xinjiang. Geoenvironmental Disasters, 2(1), 1-10.

Yonson, R. (2018). Floods and Pestilence: Diseases in Philippine Urban Areas. Economics of Disasters and Climate Change, 2(2), 107-135. doi:10.1007/s41885-017-0021-2

Yu, D. (2010). Exploring Spatiotemporally Varying Regressed Relationships: The Geographically Weighted Panel Regression Analysis. Proceedings of the Joint International Conference on Theory, Data Handling and Modelling in GeoSpatial Information Science, 134-139.

Yu, M., Li, Q., Hayes, M. J., Svoboda, M. D., \& Heim, R. R. (2014). Are droughts becoming more frequent or severe in China based on the Standardized Precipitation Evapotranspiration Index: 1951-2010? International Journal of Climatology, 34(3), 545-558. 